\title{
Unconventional Phases in Two-Dimensional Hubbard and Kondo-Lattice Models by Variational Cluster Approaches
}

\author{
Dissertation \\ zur Erlangung des mathematisch-naturwissenschaftlichen \\ Doktorgrades \\ "Doctor rerum naturalium" \\ der Georg-August-Universität Göttingen \\ im Promotionsprogramm ProPhys \\ der Georg-August University School of Science (GAUSS)
}

vorgelegt von

Benjamin Lenz

aus Haan

Göttingen, 2016 


\section{Betreuungsausschuss:}

Prof. Dr. Thomas Pruschke

Institut für Theoretische Physik

Georg-August-Universität Göttingen

Priv.-Doz. Dr. Salvatore R. Manmana

Institut für Theoretische Physik

Georg-August-Universität Göttingen

Prof. Dr. Peter E. Blöchl

Institut für Theoretische Physik

Technische Universität Clausthal

\section{Mitglieder der Prüfungskommission:}

Referent: Priv.-Doz. Dr. Salvatore R. Manmana

Institut für Theoretische Physik

Georg-August-Universität Göttingen

Korreferent: Prof. Dr. Peter E. Blöchl

Institut für Theoretische Physik

Technische Universität Clausthal

Zweiter Korreferent: Prof. Dr. Michael Potthoff (kein Mitglied der Prüfungskommission)

I. Institut für Theoretische Physik

Universität Hamburg

\section{Weitere Mitglieder der Prüfungskommission:}

Prof. Dr. Stefan Kehrein

Institut für Theoretische Physik

Georg-August-Universität Göttingen

Prof. Dr. Stefan Mathias

I. Physikalisches Institut

Georg-August-Universität Göttingen

apl. Prof. Dr. Michael Seibt

IV. Physikalisches Institut

Georg-August-Universität Göttingen

Prof. Dr. Annette Zippelius

Institut für Theoretische Physik

Georg-August-Universität Göttingen 


\section{Abstract}

Materials with strongly correlated electrons show a multitude of unconventional phenomena, such as superconductivity or magnetism, that cannot be explained in a single-particle picture and necessitate to take many-body effects into account. A route to explain the origin of these phenomena consists in constructing effective models for these materials that are then solved, often using numerical techniques. Paradigmatic models for two different classes of strongly correlated electron systems are investigated in this thesis: The Hubbard model is a paradigmatic model to describe the Mott metal-insulator transition (MIT) and the Kondo lattice model describes the low-energy physics in heavy fermion compounds. Both models are analyzed numerically using different variations of a quantum cluster technique, the variational cluster approximation (VCA).

The first part focuses on the MIT, as it is investigated experimentally in quasi-twodimensional charge-transfer salts. Its nature and associated universality class are still heavily discussed and both experiments and theoretical approaches come to different conclusions.

In this thesis, the Mott transition is investigated on an anisotropic two-dimensional Hubbard model at half-filling. By using a control parameter, which induces this anisotropy, strong evidence for Mott quantum criticality is found in weakly coupled Hubbard chains. The results at zero temperature show that the second-order critical end point $T_{c}$ of the interaction-driven metal-insulator transition can be tuned down to zero at strong anisotropy. Further results for the antiferromagnetic phase suggest a similar picture and motivate adding the anisotropy as a new axis in the phase diagram to account for a lowtemperature critical end point of the Mott transition.

The second part of this thesis focuses on unconventional phases that emerge in a paradigmatic model for heavy fermion systems, the Kondo lattice model. Using the VCA, the two-dimensional Kondo lattice model is investigated in the paramagnetic phase as well as in phases with broken symmetry. An antiferromagnetic phase at weak coupling and a phase with Kondo-singlet formation at strong coupling are analyzed. Within the antiferromagnetically ordered region, two phases with different Fermi-surface topology, which are separated by a discontinuous transition, are identified. The model is also tested for s-wave superconductivity as found recently using dynamical mean-field theory, but no indications for robust local pairing are seen within VCA. Instead, nodal d-wave superconductivity is found and analyzed in a large range of couplings and electron fillings, and its interplay with antiferromagnetic order at weak coupling is discussed. This motivates further studies on extended models that might allow for a close comparison with experiments on heavy fermion systems.

Understanding the formation of unconventional phases in strongly correlated electron systems is not only of fundamental interest, but it is also essential for designing new functional materials. Although the results of approximate cluster techniques have to be handled with care, the new aspects presented in this thesis pave the way for further investigations in this direction. 



\section{Acknowledgments}

First of all I would like to thank Thomas Pruschke ( $†$ 2016), who already supervised my master thesis and gave me the opportunity to work on this $\mathrm{PhD}$ project. He always took the time for discussions and provided many helping comments and ideas. This stayed unchanged even during his severe illness. He not only kept his interest in the project and my progress on the topic, but also continued to support me and his other students, even from the hospital. A big thank you also to Salvatore Manmana who first co-supervised me during the first years of my PhD project and then took over the main supervision. Already in the difficult situation during Thomas' illness he always took the time to supervise and support me and the other $\mathrm{PhD}$ students. He always kept an open door for discussions and shared his ideas. Especially, I also like to thank both of them for supporting my participation in several conferences, workshops, and summer schools.

I would also like to thank my collaborators Marcin Raczkowski and Fakher Assaad from the University of Würzburg who initiated the project on the Mott transition. During several video conferences and visits in Würzburg, I benefitted a lot from their insights and always enjoyed the vivid discussions. I am also thankful to my collaborators Georg Ehlers and Reinhard Noack from the University of Marburg for helpful discussions on the Mott transition. A thank you also to Benedikt Hartmann and Elena Gati from the university of Frankfurt for discussions about their experiments on layered organic charge-transfer salts. During our discussions, I learned a lot about the difficulties of measuring these materials and how to relate the results of theoretical model calculations to experimental findings. Thank you to Oliver Bodensiek for instructive discussions on s-wave superconductivity in the Kondo lattice model and on numerical details of his work on this issue. I also thank Riccardo Gezzi for discussions on superconductivity and the equation of motion technique.

Moreover, I would like to thank the members of the condensed matter theory group for all the nice discussions about various topics and for providing a lively working environment. This includes conference stays, group outings, coffee breaks in the cafeteria, and meetings in the social room. Especially, I would like to thank my (former) office mates Thomas Köhler, Alexander Tiegel, and Fabian Biebl for providing a pleasant working atmosphere in the office.

Furthermore, I would like to thank the German Research Foundation for their financial support of this project through research unit FOR1807 and to acknowledge computer support by Jürgen Holm, the GWDG, and the GoeGrid project.

The thesis would not have been this way without the comments, help with the layout, and proof reading of a lot of people. In this respect I thank Nils Abeling, Stephan Filor, Matthias Grob, Rainer Härtle, Ingo Homrighausen, Ebad Kamil, Thomas and Isabel Köhler, Mirjam Lenz, Salvatore Manmana, and Florian Sohn for their help. The present spelling errors and flaws are exclusively my responsibility.

Last but not least I would like to thank my family, especially my parents, for all the support and encouragement during my studies. 


\section{Author's Note}

The references, which are cited in the following, are listed at the end of the thesis. This bibliography includes a full list of references in alphabetical order of their labels with detailed information (author names, title of the publication, name of the journal etc.). The labels consist usually of the initials of up to four authors of the publication and two digits indicating the year of the publication or the appearance. In case of double names or special characters exceptions are possible.

Parts of this thesis have been published in a peer-reviewed journal. The corresponding reference is listed below.

$\left[\mathrm{LMP}^{+} 16\right]$ B. Lenz, S. R. Manmana, T. Pruschke, F. F. Assaad, and M. Raczkowski. Mott Quantum Criticality in the Anisotropic 2D Hubbard Model. Phys. Rev. Lett. 116, 086403 (2016)

A publication containing the results presented in chapter 5 of this thesis was submitted on 15.12.2016 and the corresponding preprint is:

[LGM16] B. Lenz, R. Gezzi and S. R. Manmana. Variational Cluster Approach to Superconductivity and Magnetism in the Kondo Lattice Model. E-print arXiv:1612.04693 (2016) 


\section{Table of contents}

\section{$\begin{array}{ll}\text { 1. Introduction } & 1\end{array}$}

1.1. Unconventional Phenomena in Strongly Correlated Electron Systems . . . 1

1.2. Layered Organic Mott Systems . . . . . . . . . . . . . . . . 3

1.2.1. Quasi-Two-Dimensional Charge-Transfer Salts . . . . . . . . . . . 4

1.2.2. Experimental Investigations of the Mott Transition . . . . . . . . . 6

1.2.3. Scaling Theories for the Mott Transition . . . . . . . . . . . . . . . 11

1.3. Heavy Fermion Systems . . . . . . . . . . . . . . . . . . . 16

1.3.1. From Kondo to Kondo Lattice Physics . . . . . . . . . . . . . . . . 19

1.3.2. The Doniach Diagram . . . . . . . . . . . . . . . 21

1.4. Outline of the Thesis . . . . . . . . . . . . . . . . . 25

$\begin{array}{ll}\text { 2. Models } & 27\end{array}$

2.1. Investigating the Mott Transition: The Hubbard Model . . . . . . . . . . . 27

2.2. Studying Quantum Magnetism: The Heisenberg Model . . . . . . . . . . . 31

2.3. Effective Description of Heavy Fermions: The Kondo Lattice Model . . . . 32

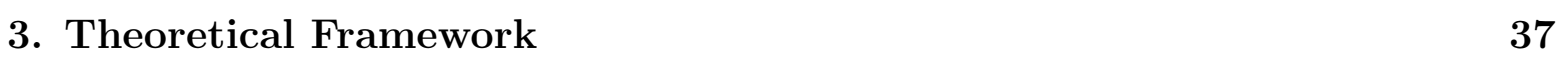

3.1. Green Functions and Cluster Perturbation Theory . . . . . . . . . . . . . 37

3.1.1. Green Function within Lehmann Representation . . . . . . . . . . . 38

3.1.2. The Gorkov-Green Function and Nambu Formalism . . . . . . . . . 43

3.1.3. Cluster Perturbation Theory: Introducing the Cluster Green Function 45

3.1.4. Treating Spins: Cluster Perturbation Theory for Spin Models . . . 50

3.2. Self-Energy Functional Theory and the Variational Cluster Approximation 58

3.2.1. Self-Energy Functional Theory . . . . . . . . . . . . . . . . 58

3.2.2. The Variational Cluster Approximation . . . . . . . . . . . . . . . 61

3.2.3. Treating Local Spin Interactions: Application of the Variational Cluster Approximation to the Kondo Lattice Model . . . . . . . . . 71

3.3. Survey of Other Numerical Approaches . . . . . . . . . . . . . . . . 73

3.3.1. The Mott Transition in Two-Dimensional Hubbard Models . . . . . 73

3.3.2. The Kondo Lattice Model . . . . . . . . . . . . . . . . . . . . . 76

4. Mott Quantum Criticality in Two Dimensions $\quad 81$

4.1. First Steps Towards the Mott Transition . . . . . . . . . . . . . . . . . . . 82

4.2. Mott Transition in the Paramagnetic Phase . . . . . . . . . . . . . . 87

4.2.1. The Phase Diagram . . . . . . . . . . . . . . . . . . . . . 88

4.2.2. Obtaining the Phase Diagram . . . . . . . . . . . . . . . . . . . . 89

4.2.3. Comparison to Cellular Dynamical Mean-Field Theory . . . . . . . 91

4.2.4. Spectral Function and Fermi Surface Pockets . . . . . . . . . . . . . 93

4.2.5. Critical Exponents at the Mott Transition . . . . . . . . . . . . . . 99 
4.3. Mott Transition into the Antiferromagnetic Phase . . . . . . . . . . . . . 103

4.3.1. Preliminary Assessment of the Inclusion of Magnetism . . . . . . 103

4.3.2. Phase Diagram . . . . . . . . . . . . . . . . . . 104

4.3.3. Obtaining the Phase Diagram . . . . . . . . . . . . . 105

4.4. Summary . . . . . . . . . . . . . . . . . . . . . . . 109

5. Unconventional Phases in the Kondo Lattice Model 111

5.1. The Paramagnetic Phase of the Kondo Lattice Model . . . . . . . . . . . . 111

5.1.1. Influence of the Intra-Cluster Hopping . . . . . . . . . . . . . 112

5.1.2. The Kondo Insulator at Half-Filling . . . . . . . . . . . . . . . . . . 113

5.1.3. Doping the Paramagnet . . . . . . . . . . . . . . 116

5.2. Magnetic Phase Diagram of the Kondo Lattice Model . . . . . . . . . . . . 118

5.2.1. The Antiferromagnet at Half-Filling . . . . . . . . . . . . . . 119

5.2.2. Doping the Antiferromagnet . . . . . . . . . . . . . . 126

5.2.3. Variation of the Intra-Cluster Hopping $t^{\prime}$. . . . . . . . . . . . . . . 134

5.3. Local Superconductivity in the Kondo Lattice Model . . . . . . . . . . . 138

5.3.1. Probing for s-Wave Superconductivity at Half-Filling . . . . . . . . 139

5.3.2. Choosing the Correct Stationary Point off Half-Filling . . . . . . . . 141

5.3.3. Absence of Local Superconductivity with a $3 \times 2$ Cluster . . . . . . 145

5.4. Nodal Superconductivity in the Kondo Lattice Model . . . . . . . . . . . . 150

5.4.1. d-Wave Superconductivity . . . . . . . . . . . . . . . . . 150

5.4.2. Competition of Antiferromagnetism and Superconductivity . . . . . 151

5.5. Summary . . . . . . . . . . . . . . . . . . . . 156

6. Conclusions 159

6.1. Mott Criticality in the Anisotropic 2D Hubbard Model . . . . . . . . . . . 160

6.2. Superconductivity and Antiferromagnetism in the Kondo Lattice Model . . 162

\section{Appendices}

A. Calculation of the Green Function in Continued Fraction Representation via the Lanczos Algorithm

B. Additional Spin Structure Factors for Heisenberg Models 169

C. Semi-Analytic Evaluation of the Self-Energy Functional 173

D. Tight Binding Theory for Néel and Collinear Order $\quad 177$

E. The Mott Transition within Dynamical Impurity Approximation $\quad 181$

F. Preliminary Studies of Local s-Wave Pairing in the Kondo Lattice Model 


\section{\begin{tabular}{l|l} 
& \\
Introduction & 1
\end{tabular}}

\section{Unconventional Phenomena in Strongly \begin{tabular}{l|l} 
Correlated Electron Systems & 1.1
\end{tabular}}

A multitude of physical properties in materials can nowadays be successfully described by standard solid-state physics [AM76, Mah90, Kit96, KV04]. Especially materials like simple metals, semiconductors, and insulators were understood quite early, as a description by non-interacting or weakly interacting electrons within band theory turned out to be sufficient [IFT98]. However, materials where d- or f-electron bands are only partially filled, for example in transition metal oxides, were not well described by these theories at all.

The reason consists in the strong spatial confinement of electrons in these shells, which results in a strong Coulomb repulsion between them. The strong interaction between the electrons and the resulting strong electron-electron correlation has to be included in a theoretical description in order to explain the physical properties of these materials properly. In these systems, the potential energy due to the Coulomb repulsion between the electrons is at least of the order of their kinetic energy, which makes the use of efficient techniques in the weak and strong interaction limit impossible. Even modern single-particle methods like density functional theory [JG89] or effective static mean-field treatments of the correlations are not successful [IFT98, KV04]. Although the importance of interactions between electrons was already recognized in 1937 and a lot of work has been done since, see e.g. the review by Imada et al. [IFT98], strongly correlated electron systems still bear open questions.

The electronic correlations lead to a plethora of exotic phases with unconventional ordering at low temperatures [KV04]. In the following, a few striking phenomena that emerge due to these correlations are briefly mentioned; aspects of these phenomena will be investigated in this thesis.

\section{Mott transition}

The Mott metal-insulator transition, as it can be observed in transition metal oxides, was one of the first phenomena that could be linked to strongly correlated electrons [IFT98]. Nevertheless, a lot of questions about its nature remained open [IFT98] and the interest in its universality class has raised again recently as experimental improvements now allow the investigation of low-dimensional prototypical materials for this transition [KMK05, KK11, AJKW $\left.{ }^{+} 15\right]$.

\section{Kondo effect}

The Kondo effect [Kon64] and its lattice analog, the lattice Kondo effect [Col07], also emerge due to strong interactions between electrons. Especially the latter is essential to understand the physics of heavy fermion systems [Kon64, Hew93, Ste84, Col15a], where 
Kondo entanglement and its breakdown is the key to understanding the nature of quantum critical points [GSS08, SS10].

\section{Magnetism}

Different kinds of magnetic ordering like antiferromagnetism are phenomena that are known for long, but in order to explain their microscopic emergence a proper treatment of electronic interactions is essential [Blu01]. Besides long-known magnetic phases more exotic magnetic phases can emerge, especially if different magnetic orderings are competing in the system, for example if geometrical frustration or disorder is present. These competitions can lead to new phases of matter such as quantum spin liquids $\left[\mathrm{PBB}^{+} 11, \mathrm{YHW} 11, \mathrm{FMI}^{+} 15\right]$.

\section{Superconductivity}

Unconventional superconductivity also emerges due to strong electronic correlations in a lot of materials such as heavy fermion systems [SAB ${ }^{+} 79$, SS10, $\mathrm{SSW}^{+} 13$, SW16], cuprate superconductors [BM86, Sca95, Leg06], or organic charge-transfer salts [BW13]. In contrast to conventional superconductors, where pairing is mediated by phonons, the pairing is in this case mediated through complicated many-body processes, which ask for explanation. Also the interplay with other phenomena like magnetism or the Mott transition is still being investigated heavily $\left[\mathrm{NSW}^{+} 10, \mathrm{SAF}^{+} 11\right.$, GSGB15, SW16].

In this thesis, the Mott transition and antiferromagnetism are investigated within the anisotropic Hubbard model. This is detailed in chapter 4. A class of materials, where the nature of the Mott transition is still heavily discussed, are organic charge-transfer salts. They will be introduced in section 1.2 to set the stage for the investigation of this anisotropic Hubbard model later. The phenomena of Kondo singlet formation, antiferromagnetism, unconventional superconductivity, and their interplay are studied in the Kondo lattice model in chapter 5 . This is a paradigmatic model for heavy fermion materials, which are introduced in section 1.3. In an excursus using a less elaborate technique, quantum magnetism is furthermore analyzed within effective spin models in section 3.1.4.

Interestingly, the phase diagrams of organic charge transfer salts and heavy fermion compounds are quite similar. They do not only both contain most of the above mentioned emergent phenomena, but are also both discussed in the context of quantum critical behavior. At a quantum critical point (QCP), a continuous phase transition is driven by quantum fluctuations only, which is strictly speaking only possible at zero temperature as thermal fluctuations are then absent [Voj03]. However, remnant quantum fluctuations are expected to persist even at non-zero temperatures and are used as an indicator for a QCP in experiment. Whereas the (seemingly) quantum critical behavior in the crossover regime of organic charge transfer salts is still heavily discussed $\left[\mathrm{FMT}^{+} 15, \mathrm{AJKW}^{+} 15\right]$, different quantum critical points have been found in certain heavy fermion compounds [GSS08, SS10, GSGB15, SW16]. In order to answer the question of quantum criticality in the former case, the determination of the universality class of the phase transition is a subject of current research.

Lots of the models that are nowadays investigated for these emergent phenomena are lowdimensional. These also include the two-dimensional (2D) models that are investigated in this thesis. Heavy fermion systems, cuprates, or certain organic charge-transfer salts 
are just a few prominent examples that can be described by quasi-2D models. In these cases, the strongly interacting electrons are spatially confined, e.g. to a two-dimensional plane. Due to this confinement and at low temperatures, quantum effects become strong and dominate the emergent properties such as the phenomena listed before.

From an academic point of view, low-dimensional systems are interesting as in addition to strong quantum fluctuations additional effects like geometrical frustration become important. Besides the aforementioned layered systems, there exists another prominent experimental realization of such two-dimensional lattices: In so-called ultracold gases, degenerate fermionic or bosonic quantum gases are trapped in optical traps and cooled down to temperatures in the nanokelvin regime [Blo08, BDZ08, WHR15]. Thereby, strongly interacting bosonic and fermionic systems can be designed and subsequently investigated in experiment. In comparison to layered compounds, which also show quasi-2D physics, the good experimental control over these systems allows for nearly one-to-one comparison between theory and experiment.

Within this thesis the variational cluster approximation (VCA) is used to investigate the anisotropic 2D Hubbard and the 2D Kondo lattice model. In case of the isotropic oneand two-dimensional Hubbard model this technique has already been successfully used and is quite well understood. As it is capable of treating both antiferromagnetism and the Mott transition properly, it is an appropriate technique to study the dimensional crossover between one and two dimensions. It is shown that VCA can also be used to investigate the Kondo lattice model, where electrons interact locally with static magnetic moments. Afterwards, the technique is used to study the aforementioned phases individually as well as their interplay. In an excursus, low-dimensional pure spin systems are treated with an adaptation of cluster perturbation theory. The recurrence to this less elaborate quantum cluster technique is necessary, as it was shown by Filor and Pruschke that a modified variational cluster approach for spin systems has severe limitations [FP14].

In sections 1.2 and 1.3, the stage for the two main results of this thesis is set by introducing systems that can be described by the effective models used later. Then, section 1.4 gives an outline of this thesis.

\section{\begin{tabular}{l|l} 
Layered Organic Mott Systems & 1.2
\end{tabular}}

In this section, two different layered organic materials, known experimental results for and theoretical approaches to the Mott transition in these materials, as well as some of the open and heavily debated questions that remain in this field of research are introduced.

Before discussing some of the aspects of the Mott transition in organic conductors in detail, a brief motivation for studying these systems even without focussing on the Mott transition is given in the following.

The family of quasi-two-dimensional organic charge-transfer salts are for a number of reasons an interesting field of research. Most intriguing is the plethora of different phases with broken symmetries at low temperatures, including different superconducting, magnetic, insulating, and metallic phases. Even some more exotic phases such as a Fulde-FerrellLarkin-Ovchinnikov (FFLO) superconducting state [BW13] or possibly even a realization of a quantum spin liquid ground state $\left[\mathrm{YNO}^{+} 08, \mathrm{YNK}^{+} 09, \mathrm{YNS}^{+} 10\right]$, just to name a few, 
have been found. The experimental control of the systems is good, which is why these systems are not only used to determine transitions between these phases, but also to closely investigate phenomena like BEC-BCS crossover in the superconducting phase or spinon-holon separation in quasi-one-dimensional (quasi-1D) salts. Due to this variety of different phases, organic salts are often compared with three-dimensional layered heavy fermion materials or high-temperature cuprate superconductors, which surprisingly show a similar phase diagram.

Compared to the cuprates, most of the energy scales are roughly of a magnitude smaller and the systems can be well controlled experimentally. Specifically, it is possible to tune the bandwidth of these salts by applying external pressure of a feasible size and to thereby gain good control on the measurements. Nevertheless, some of the questions one likes to address in these compounds remain challenging in experiments as precise measurements at high pressures and low temperatures are required. In contrast to heavy fermion systems, where a quantum critical point (QCP) is found for a lot of compounds although it is sometimes hidden [SS10], no such QCP has been found so far for organic charge-transfer salts. Still, recent experimental works claim to have measured quantum critical behavior at temperatures above the second-order end point $T_{c}$ of a metal-insulator transition line [KMK05], which triggered a lot of subsequent works and raised the question of the nature of this transition.

Another interesting observation along this line concerns the critical end point $T_{c}$ of several materials that are expected to show a Mott transition. When comparing the critical end point $T_{c} \approx 450 \mathrm{~K}$ of Cr-doped $V_{2} \mathrm{O}_{3}$, which is a three-dimensional crystal, with those of two-dimensional organic salts such as $\kappa-(\mathrm{BEDT}-\mathrm{TTF})_{2} \mathrm{X}\left(T_{c} \approx 40 K\right)$, the question arises, whether reducing the dimensionality of the system further might still lower $T_{c}$ [RA12].

\section{Quasi-Two-Dimensional Charge-Transfer Salts $\mid 1.2 .1$}

Although the organic charge-transfer salts differ in their effective crystal structure and resulting phase diagrams, they all consist of two different parts, which build up a periodic layered structure. In this section, two different types of salts are introduced, which are the protagonists of the discussion about the universality class of the Mott transition (see next paragraph). Nevertheless, by selecting the so-called (BEDT-TTF) ${ }_{2} \mathrm{X}$ salts and $\mathrm{Pd}(\mathrm{dmit})_{2}$ compounds, one chooses not only Mott systems, but also material classes with a rich phase diagram, which turned these materials into a playground for the investigation of exotic phases.

Here, the focus will be on the Mott metal-insulator transition. First, one should address the question, which conditions have to be fulfilled to obtain a molecular conductor. For both compounds, charge carriers have to be created and subsequently delocalized. The (BEDT-TTF $)_{2} \mathrm{X}$ salts, where BEDT-TTF stands for bis(ethylenedithio)tetrathiafulvalene and $\mathrm{X}$ denotes some monovalent polymeric anion, fulfill these two conditions. BEDT-TTF, which is shown in the left panel of figure 1.1 and often further abbreviated by ET, is a charge donor and builds up conducting layers. On the other side $\mathrm{X}$ is a monovalent charge acceptor and is insulating in itself. In $\mathrm{Et}_{x} \mathrm{Me}_{4-x} \mathrm{Z}\left[\mathrm{M}(\mathrm{dmit})_{2}\right]_{2}$ this is also the case: Here, dmit stands for 1,3-dithiole-2-thione-4,5-dithiolate and builds together with a metallic atom $\mathrm{M}$ (in the following palladium) a molecule with a formal 
charge of $-1 / 2$. The second part of the compound consists of ethyl $\left(C_{2} H_{5}^{-}\right.$, Et $)$and methyl $\left(\mathrm{CH}_{3}^{-}, \mathrm{Me}\right)$ groups and $\mathrm{Z}$ stands for $\mathrm{N}, \mathrm{P}, \mathrm{As}$, or $\mathrm{Sb}$, so that it has an effective charge of +1 . These metal dithiolene complexes have been studied extensively [Kat04], but the discussion of the universality class of the Mott transition focussed on EtMe $\left.\mathrm{Sb}_{3} \mathrm{Sbd}(\mathrm{dmit})_{2}\right]_{2}$ $\left[\mathrm{FMT}^{+} 15\right]$ and $\mathrm{EtMe}_{3} \mathrm{P}\left[\mathrm{Pd}(\mathrm{dmit})_{2}\right]_{2}\left[\mathrm{AJKW}^{+} 15\right]$. In both quasi-2D organic compounds, by joining both parts, charge carriers are created via oxidation and delocalized by the stacking.
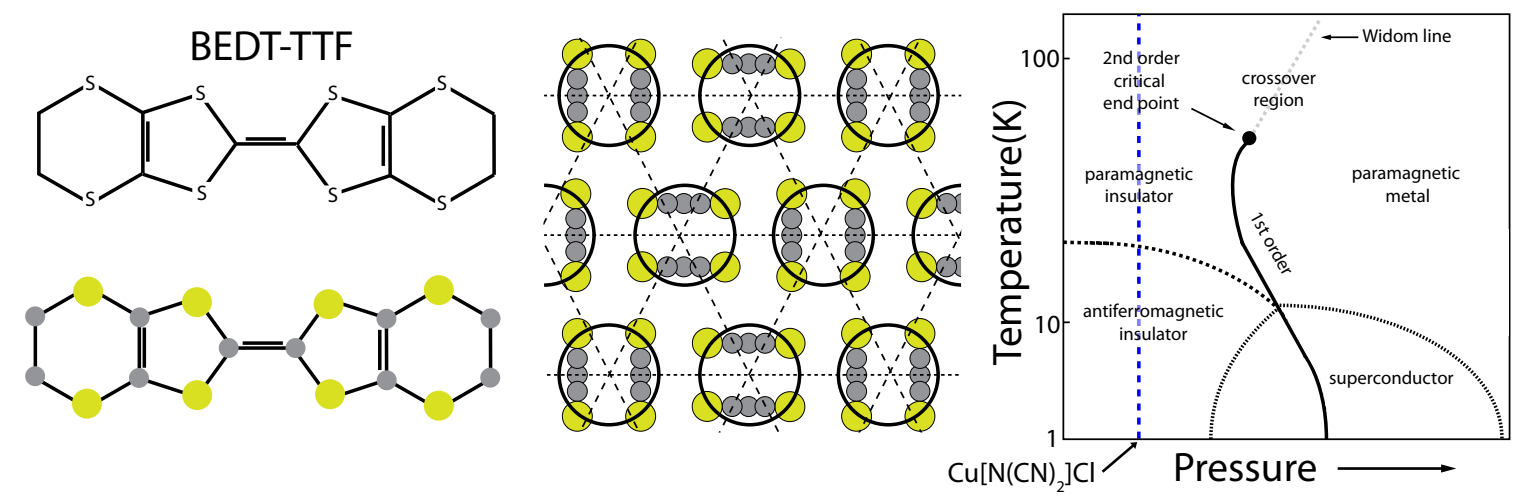

Figure 1.1: Structural formula and sketch of BEDT-TTF (left panel) and a sketch of the effective triangular lattice motivated by a lateral cut through the BEDT-TTF layer of an $\kappa$ salt (middle panel). The right panel sketches a generic phase diagram of $\kappa$-salts, the position of $\kappa-(\mathrm{ET})_{2} \mathrm{Cu}\left[\mathrm{N}(\mathrm{CN})_{2}\right] \mathrm{Cl}$ is indicated by a dashed blue line. Figures adapted from references [KMK05, KK11].

Depending on the choice of $\mathrm{X}$ and $\mathrm{Z}$, the lattice structure and electron filling of the compounds change dramatically. One of the common structures is the so-called $\theta$-phase, where the (ET) molecules do not dimerize and the system is at quarter filling. Other structures are for example the $\beta$ - and $\beta^{\prime}$-phases, where the electron filling of the system is at three quarters [Kat04]. However, the discussion of the Mott transition focussed on salts that are in the $\kappa$-phase, which is sketched in figure 1.1. This phase is obtained if two of the (ET) molecules dimerize and can be mapped to an effectively half filled triangular lattice, which is usually anisotropic [KK11].

Within some downfolding procedure based on density functional theory (DFT) or Hückel molecular orbital calculations, one often maps these systems to Hubbard models, which might even contain non-local interactions. Due to the variety of anions which can be used, it is possible to realize effective Hubbard models with different hopping parameters and Coulomb repulsions, especially when a second anion is used to dope the system chemically.

Although the assumption of dimerization and the subsequent mapping to a triangular lattice is widely used and well accepted, a recent DFT + RPA calculation has shown that this simplification is not well justified for a number of compounds. At least for the symmetry of the superconducting state at low temperatures, the treatment of the full, not-dimerized system was shown to be crucial [GAJV16].

\section{Tuning Across the Mott Transition}

There are two different ways how Mott transitions can be triggered, namely fillingcontrolled and bandwidth-controlled Mott transitions. In this section, the most common 
ways to achieve both of these transitions are briefly introduced. However, most of the studies that investigate the universality class of the transition are done for the bandwidthcontrolled transition, which is also shown in the right panel of figure 1.1.

Although the integrated molecular functionality of realizing different lattice structures offers quite some flexibility to tune the system chemically by molecular packing, one has to bear in mind that additional structural changes can occur as function of temperature or pressure. Besides structural transitions, the variation of pressure more generally changes the bandwidth of the systems, which allows one to vary the ratio of Coulomb repulsion $U$ and bandwidth $W$ of the effective Hamiltonian by tuning an external parameter. In addition, it is possible to replace one or several of the hydrogen atoms of the ethylene end-groups by deuterium, which acts like chemical pressure and can move the materials towards Mott insulating behavior [KMK97]. It is precisely this comparably easy tuneability of the bandwidth that allows to investigate the phase diagram and in particular the Mott transition. The aforementioned $\kappa$ - and dithiolene-compounds can be chemically tuned to be at ambient pressure and low temperatures close enough to the metal-insulator transition that an additional increase of (external) hydrostatic pressure allows for moving across the Mott transition.

Another possibility to tune across the Mott transition line is to change the Coulomb repulsion $U$ by adding additional carriers to the system and enhance the screening. This can be achieved by using the principle of a field-effect transistor to electrostatically dope the system $\left[\mathrm{ABDV}^{+} 06\right]$. Recently, this technique has also attracted interest for application on organic Mott insulators as it allows for nearly continuous tuning of the carrier density and thereby of the resulting field-induced Mott transition $\left[\mathrm{KYT}^{+} 09, \mathrm{KSE}^{+} 16\right]$.

Besides the bandwidth- and bandfilling-controlled Mott transition, there is a third way of inducing a Mott transition that makes use of a glasslike structural ordering [HMS14]. When cooling the sample, one usually tries to use a small cooling rate, which allows the ethylene end groups to order in a so-called eclipsed configuration. There exists a second, so-called staggered configuration of the ethylene groups, which has slightly higher energy. When cooling under the glass transition temperature $T_{g}$, a finite fraction of molecules stays in the unfavorably staggered configuration and causes structural disorder. However, raising the cooling rate changes the ratio of molecules in the two different configurations and in the end results in an anisotropic change of the in-plane lattice parameters at $T_{g}$. This amounts to a smaller bandwidth, which can be used to tune over the metal-insulator transition line [SYS ${ }^{+}$05, HMS14].

The latter possibility of investigating the Mott transition has to be considered carefully as it is closely related to the notion of disorder-driven or Anderson-Mott transitions. Instead of using the cooling rate of the sample to induce a glasslike ordering, a common way of introducing disorder to the system consists in irradiating the sample with $\mathrm{x}$-rays $\left[\mathrm{SOY}^{+} 07\right]$.

\section{Experimental Investigations of the Mott Transition $\mid 1.2 .2$}

Before comparing the different experimental findings regarding the critical exponents and thereby the universality class of the Mott transition, in this section, I give a brief overview over the different experimental techniques that are used in these works. Not only the experimental techniques, but also the measured materials and the investigated regions of the phase diagram differ in most of the studies. In the end, the critical exponents 
also depend on the assumptions entering the scaling functions, which are used to extract critical exponents. All this leads to different interpretations of the data and different explanations of the nature of the Mott transition.

What to measure? Investigating the Mott transition experimentally can be done by measuring different quantities. Obviously, measuring the conductivity as a function of temperature and some additional parameter such as the pressure is the most evident to determine a metal-insulator transition, see e.g. [KMK05, $\left.\mathrm{FMT}^{+} 15, \mathrm{AJKW}^{+} 15\right]$. It is even possible to obtain information from the resistance fluctuations (so-called fluctuation spectroscopy), where, in addition to the resistance, the resistance noise power spectral density is also investigated $\left[\mathrm{HZP}^{+} 15\right]$.

Another approach consists in using nuclear magnetic resonance (NMR) measurements to investigate, e.g. $\kappa$-ET salts, where the two central carbon atoms in ET have been replaced by ${ }^{13} \mathrm{C}$ isotopes, as done by Kagawa et al. [KMK09]. To investigate the charge-carrier dynamics near the Mott transition, especially their competition between itineracy and localization, it is also possible to use optical conductivity measurements [DG02]. Besides an analysis of the electronic properties of charge-transfer salts $\left[\mathrm{MDD}^{+} 08\right]$, it is also possible to explain effects of the vibrational lattice modes that are not covered by a purely electronic model [DLacP $\left.{ }^{+} 16\right]$.

The techniques mentioned so far "only" probe electronic quantities of the system. Thermodynamic quantities can also provide a route to determine the universality of the Mott transition. de Souza et al. investigated the thermal expansion of a $\kappa$ - $(\mathrm{ET})_{2} \mathrm{X}$ organic conductor and observed lattice anomalies near the Mott transition [dSBS $\left.{ }^{+} 07\right]$. Close to the transition they extracted the critical exponent $\alpha$, which is related to the specific heat via $C \propto|t|^{-\alpha}$. In a study by Abdel-Jawad et al. [AJKW $\left.{ }^{+} 15\right]$, the thermoelectric power ${ }^{1}$ and the conductivity have been measured as a function of pressure and temperature. In contrast to the previous conductivity study, which focused only on the metallic side of the transition to extract critical exponents [KMK05], in [AJKW $\left.{ }^{+} 15\right]$ both sides including the insulating one were measured. However, one should note that thermal-expansion measurements are capable of measuring lattice effects, which seem to become important close to the Mott transition and which are not captured by a purely electronic model. Hence, the choice of the scaling function is crucial, see section 1.2.3.

Where to measure? In low-dimensional systems, superconductivity and magnetically ordered phases often appear, which do not help in understanding the nature of the Mott transition. For this reason, one concentrates on temperatures close to the critical end point $T_{c}$, where the metal-insulator transition is indeed of Mott type. This means that one goes from a paramagnetic Mott insulator through a first-order transition into a parametallic metallic phase by increasing pressure $\left[\mathrm{AJKW}^{+} 15\right]$. This is sketched for the case of $\kappa-(\mathrm{ET})_{2} \mathrm{Cu}\left[\mathrm{N}(\mathrm{CN})_{2}\right] \mathrm{Cl}$ in the right panel of figure 1.1. In the temperature region between the second order critical end point and the onset of antiferromagnetic insulating and superconducting phases at very low temperatures, the system shows a first order Mott transition between paramagnetic insulator and paramagnetic metal.

Another possibility to obtain information about the transition is to investigate the crossover regime, that is $T>T_{c}$, where signatures of quantum criticality have been found recently $\left[\mathrm{KMK}_{0}, \mathrm{FMT}^{+} 15\right]$. In this approach, the resistivity $\rho$ is measured as a function of external pressure $P$ and temperature $T$. The inflection point of the $\log \rho(P, T)$ versus $\mathrm{P}$

\footnotetext{
${ }^{1}$ Also known as Seebeck coefficient.
} 
curve is determined for different temperatures above $T_{c}$ and forms the so-called Widom line $^{2}$. Using the Widom line to distinguish insulator from (bad) metal in the crossover region, one can investigate the scaling in both regimes and try to deduce critical exponents $\left[\mathrm{FMT}^{+} 15\right]$.

It might seem odd not to investigate the (first-order) Mott transition close to the critical point, but to measure in the crossover region instead. The reason for this is mostly the inability to do high-precision measurements in the critical region [AJGH16]. In general, it is experimentally challenging to control the very high pressures at low temperatures, especially when pressure sweeps have to be performed. Currently, most experimental setups allow only for a controlled decrease in pressure, but not of a controlled increase, which makes a direct measurement of hysteresis as a function of pressure impossible. Further limitations are evoked by structural transitions of the compound at low temperatures. Despite these challenges, the main problem for the organic charge-transfer salts comes from the coolant. The most precise measurements have been obtained by using liquid helium as a cooling medium. However, at low temperatures and pressures of up to several hundred $\mathrm{MPa}$, helium undergoes a solidification transition. On the one hand, this transition leads to a more or less uncontrolled change of pressure, which renders precise measurements at the transition impossible. On the other hand, pressure measurements within the solid phase of helium are also not precise enough to obtain a reliable and meaningful phase transition line for the organic salts. Unfortunately, at the pressures needed to investigate the critical end point of most of the organic salts, solidification of helium sets in already at temperatures above $T_{c}$, such that measurements in the crossover region are the only possibility left to obtain precise measurements.

Fate of the Fermi surface. Despite the high level of control in experiments, which is a clear advantage of molecular quasi-2D Mott systems, there are also some peculiarities, which restrict the experimental techniques that can be used. Especially for the investigation of the Fermi surface of conducting molecular systems, one would like to resort to angular-resolved photo-emission spectroscopy (ARPES), that had lead to valuable insights in other fields like the one of heavy fermions within the last decades [KB06, LVY $\left.{ }^{+} 12\right]$. Unfortunately, it is difficult to grow large crystals and in practice impossible to cleave the surface of such crystals, which renders ARPES an inappropriate technique for organic molecular conductors.

However, if one is interested in closed regions of the Fermi surface, such as so-called electron and hole pockets, which are discussed in section 4.2, de Haas-van Alphen (dHvA) or Shubnikov-de Haas measurements can provide insights (see e.g. [SSB+00]), although the dHvA effect is not a unique indicator for the presence of a Fermi surface [KC15].

\section{Universality Class of the Mott Transition Revisited by Experiments}

Before turning to quasi two-dimensional materials, it is interesting to first of all revisit the results of Limelette et al., who studied the Mott transition in Cr-doped $\mathrm{V}_{2} \mathrm{O}_{3}$ [LGJ ${ }^{+} 03$ ], which is a three-dimensional (3D) compound. They measured conductivity as a function of pressure and temperature and found a first-order metal insulator transition with a critical end point of $T_{c} \approx 450 \mathrm{~K}$. By scaling the conductivity with pressure and temperature along different paths in the phase diagram, they were able to extract critical exponents. Away

\footnotetext{
${ }^{2}$ Going back to B. Widom [Wid72] and first used in the context of Mott insulators by Sordi et al. [SSHT12].
} 
from the critical point, they found mean-field universality and in its immediate vicinity 3D Ising universality. In their paper, they back up these experimental findings by calculations using dynamical mean-field theory (DMFT), which is assumed to be valid in 3D.

Although the use of DMFT and the small region very close to the transition that was used to extract Ising exponents can be questioned, there are some more fundamental points that may be criticized. For example, it may be questioned, why the scaling laws have only been based on the conductivity measurements on the conducting side and not on the data, which were obtained in the insulating phase ${ }^{3}$.

On the other side, it was assumed that eletronic degrees of freedom dominate the transition and therefore only the conductivity entered the scaling function. As also stated by the authors, the effect of lattice degrees of freedom should be included in order to confirm their finding of 3D Ising universality.

From considering this 3D compound, but also from more general considerations, which are based on symmetry arguments for the half-filled single-band Hubbard model [CCFR79], it was expected that the quasi-2D charge-transfer salts would also show Ising universality [KLR00, ON03, GFC04].

This changed in 2005, when conductivity measurements on quasi-2D $\kappa-\mathrm{ET}$ salts in the group of K. Kanoda revealed critical exponents that did not only contradict 2D Ising universality, but also did not match any known universality class [KMK05]. In their studies, they used the same experimental setup as Limelette et al. to measure conductivity as a function of pressure and temperature in $\kappa-(\mathrm{BEDT}-\mathrm{TTF})_{2} \mathrm{Cu}\left[\mathrm{N}(\mathrm{CN})_{2}\right] \mathrm{Cl}$ (from here on denoted by $\kappa-\mathrm{Cl}) . \kappa-\mathrm{Cl}$ is a Mott insulator at ambient pressure and undergoes a firstorder metal-insulator transition when pressure is applied. It is important to note that the desired transition can only be observed within a narrow temperature region, as the firstorder transition ends at a critical temperature $T_{c} \approx 39.7 \mathrm{~K}$ and as the insulator becomes antiferromagnetic and the metal an unconventional superconductor if the temperature is chosen too small.

As in the previous study by Limelette et al., they investigated the conductance behavior on the metallic side of the phase diagram close to the transition and found power laws close to the critical end point. From the slopes of logarithmic plots of conductance against temperature pressure they determined the critical exponents to be $(\delta, \beta, \gamma)=(2,1,1)$. They avoided the region in the immediate vicinity of the end point as there, the results very much depend on the precise value of $T_{c}$ and small errors in the determination of $T_{c}$ strongly affect the exponents.

Subsequent nuclear magnetic resonance (NMR) measurements of the same group on the very same $\kappa-\mathrm{Cl}$ salt seemed to confirm these exponents [KMK09]. By identifying metallic and insulating resonance peaks in NMR spectra and comparing their volume fractions as a function of pressure, they were able to detect the metal-insulator transition. They also measured the nuclear spin-lattice relaxation rate divided by temperature $1 / T_{1} T$, which is connected to the dynamical spin susceptibility. Thereby, they could investigate a quantity that measures antiferromagnetic fluctuations and found logarithmic scaling of $\Delta \frac{1}{T_{1} T}$ with pressure $P$, which even allowed them to extract critical exponents. They assumed that the criticality in transport and magnetism is identical and concluded that even the

\footnotetext{
${ }^{3}$ For such a study on a dithiolene-compound see [AJKW $\left.{ }^{+} 15\right]$.
} 
magnetic Mott criticality might have the same nature.

Thermal-expansion measurements in the group of $M$. Lang lead to the observation of an anomalous lattice response at the Mott transition [dSBS $\left.{ }^{+} 07\right]$. A subsequently proposed scaling theory for this data suggests that the material instead belongs to 2D Ising universality class [BdSL10].

Recently, the group of K. Kanoda also claimed to have found quantum criticality of the Mott transition for other organic salts $\left(\kappa-(\mathrm{ET})_{2} \mathrm{Cu}_{2}(\mathrm{CN})_{3}, \kappa-(\mathrm{ET})_{2} \mathrm{Cu}\left[\mathrm{N}(\mathrm{CN})_{2}\right] \mathrm{Cl}\right.$, and $\left.\mathrm{EtMe}_{3} \mathrm{Sb}\left[\mathrm{Pd}(\mathrm{dmit})_{2}\right]_{2}\right)\left[\mathrm{FMT}^{+} 15\right]$. As these compounds have different low-temperature phase diagrams, but show the same critical behavior for $T>T_{c}$, they concluded that the Mott transition is independent of the broken symmetry states at zero temperature. The unconventional Mott criticality should be a more general phenomenon instead. Furthermore, the fact that the critical end point of the transition is relatively low suggests quantum effects to become important. Instead of a physical picture building on classical phase transitions [Ima05, MI07, IMY10], including quantum effects seems to be necessary.

Nearly at the same time, a combined study of conductance and thermo-power measurements by Abdel-Jawad et al. showed instead that EtMe $\mathrm{E}_{3} \mathrm{P}\left[\mathrm{Pd}(\mathrm{dmit})_{2}\right]_{2}$ falls into 2D Ising universality class [AJKW $\left.{ }^{+} 15\right]$. In contrast to the compounds studied by Kagawa et al., the critical end point of $\mathrm{EtMe}_{3} \mathrm{P}\left[\mathrm{Pd}(\mathrm{dmit})_{2}\right]_{2}$ still falls within the compressible region of helium such that the first-order metal-insulator transition up to the critical end point can be investigated directly. One of their key points is that it is important to fit the exponent only in the close vicinity of the transition. This is precisely the region that Kagawa et al. spared out in their 2005 study due to ambiguity of the results. Another important issue is that if the conductivity is assumed to depend on the inverse coherence scale $1 / \xi$ instead of the order parameter, even the results of Kagawa et al. [KMK05] give exponents of $\nu_{c} \approx 1 / 2$ and $\nu \approx 1$. Thereby, they would support Ising universality class. This conceptual difference is discussed in more detail in the following section 1.2.3.

Finally, Abdel-Jawad et al. show that there is some ambiguity for defining the metalinsulator transition in the crossover region $T>T_{c}$, depending on whether conductivity, Seebeck coefficient, or the pressure derivative of the conductivity is considered. Therefore, using the crossover region to deduce critical scaling may lead to different results, depending on which quantity is used to determine the transition.

To conclude, the question of the universality class of the Mott transition in charge-transfer salts remains open. Investigations of several quantities that should allow a determination of the universality class lead to different answers, see table 1.1. Even measuring the same quantity allows for different interpretations. This is at least partially due to the assumptions that enter the used scaling functions. A brief overview of scaling theories for the Mott transition is given in the following subsection.

A survey of numerical approaches to the Mott transition in two-dimensional Hubbard models will be given in subsection 3.3.1. 


\begin{tabular}{|l|l|l|}
\hline Technique/ Measurement & Critical exponents & Ref. \\
\hline \hline Conductivity & $\beta=1, \gamma=1, \delta=2$ & {$[\mathrm{KMK05}]$} \\
NMR & $\delta=2$ & {$[\mathrm{KMK09]}$} \\
Conductivity & $z \nu=0.49 \pm 0.01,0.62 \pm 0.02,0.68 \pm 0.04$ & {$\left[\mathrm{FMT}^{+} 15\right]$} \\
\hline Thermal expansion & $\alpha=0.8 \pm 0.15$ & {$\left[\mathrm{dSBS}^{+} 07\right]$} \\
\hline Thermal expansion & $(1-\beta) /(\beta+\gamma)=7 / 15,1 /(\beta+\gamma)=8 / 15$ & {$\left[\mathrm{BdSL}_{10}\right]$} \\
Conductivity \& & $\nu_{c}^{\text {met }}=0.52 \pm 0.03, \nu_{c}^{\text {ins }}=0.50 \pm 0.03$, & {$\left[\mathrm{AJKW}^{+} 15\right]$} \\
thermal expansion & $\delta \beta=15 / 8$ & \\
\hline
\end{tabular}

Table 1.1: Summary of the critical exponents as they have been extracted in the experimental studies mentioned in the text. The first three studies support unconventional criticality with $(\alpha, \beta, \gamma, \delta)=(-1,1,1,2)$, the last two suggest $2 \mathrm{D}$ Ising universality with $(\alpha, \beta, \gamma, \delta)=$ $(0,0.125,1.75,15)$.

\section{Scaling Theories for the Mott Transition 1.2 .3}

Critical exponents. In the following, the concept of criticality is briefly introduced, based on references [KGH ${ }^{+} 67$, Gol92, Voj03]; a thorough, more detailed treatment of critical phenomena can be found therein.

Phase transitions are often classified into first-order and continuous transitions ${ }^{4}$. In firstorder transitions, one finds a coexistence region of both phases around the transition temperature, whereas in a continuous phase transition there is no coexistence. The point where the system changes from one phase to the other is called critical point. In continuous phase transitions, at the critical point both spatial and temporal fluctuations of the order parameter diverge in the disordered phase. The characteristic size of these fluctuations is given by the correlation length $\xi$ and the correlation time $\tau$. As both $\xi$ and $\tau$ are infinite at the critical point, the system is scale-invariant and can be described by measuring observables as a function of external parameters that can be tuned. The critical exponents, which can be extracted from the resulting power laws then characterize the system. Depending on the quantity that is measured it is possible to extract seven critical exponents in total, four thermodynamic exponents, two exponents $\nu$ and $\eta$ of the correlation length and the correlation function, and the dynamic exponent $z$, which is connected with the divergence of correlation time $\tau$. In the end, it is assumed that all those critical exponents characterize the phase transition and allow for a classification of several phase transitions into universality classes. Systems within one universality class show the same symmetry of the order parameter and space dimensionality.

Despite having a set of critical exponents, a lot of these exponents are connected via scaling laws. For example, the thermodynamic exponents can be obtained from the free energy, which usually only depends on two exponents [Voj03, Gol92, Wid65]. Hence, the four thermodynamic critical exponents can be connected by two scaling laws, e.g. by Rushbrooke's scaling law [EF63, Rus63]

$$
\alpha+2 \beta+\gamma=2
$$

\footnotetext{
${ }^{4}$ Despite the same naming, this should not be confused with the Ehrenfest classification scheme for classical transitions, which is based on discontinuities of derivatives of the thermodynamic free energy.
} 
which connects the critical exponent of the specific heat $(\alpha)$ with those of the order parameter $(\beta)$ and of the susceptibility $(\gamma)$ and by Widom's scaling law

$$
\beta(\delta-1)=\gamma .
$$

The latter also involves the exponent of the critical isotherm $\delta$. For the correlation function exponents, there also exist two scaling laws, namely Josephson's scaling laws

$$
\nu d+\alpha=2
$$

and

$$
\gamma / \nu+\eta=2
$$

In contrast to the Rushbrooke law, there are not only thermodynamic quantities involved, but also the critical exponent of the correlation length $(\nu)$ and the one of the scaling function $(\eta)$. However, the dimension $d$ explicitly enters the scaling law, which is a drastic difference to the previously shown laws. These scaling laws involving $d$ are called hyperscaling relations. In contrast to these six critical exponents, the dynamical exponent $z$ is not connected to the other (static) exponents.

Transition driven by the order parameter. Although the universality class of quasi$2 \mathrm{D}$ organic charge-transfer salts is currently one of the fields where the nature of the Mott transition is discussed intensely, the starting point for the discussion were the studies on $3 \mathrm{D}$ systems, such as $\mathrm{V}_{2} \mathrm{O}_{3}$ [LGJ $\left.{ }^{+} 03\right]$. In this paper by Limelette et al. it was assumed that the conductivity $\sigma$ at the critical end point is proportional to the order parameter. At constant critical temperature $T_{c}$, the conductivity $\sigma$ as a function of pressure $P$ should therefore scale as $\sigma\left(T_{c}, P\right)-\sigma_{c} \propto\left(P-P_{c}\right)^{1 / \delta}$, where all quantities at the second-order critical end point are denoted by the index 'c'. Furthermore, the authors defined the pressure limit of the spinodal insulating phase $P_{I}(T)$ as the highest pressure, at which insulating phase could be obtained when increasing the pressure. Using this quantity, it was also possible to scale the conductivity with temperature via $\sigma\left(T, P_{I}(T)\right)-\sigma_{c} \propto$ $\left(T_{c}-T\right)^{\beta}$. Thereby, the critical exponents $\beta$ and $\delta$ could be fitted from the data and showed mean-field values of $(\delta, \beta)=(3,0.5)$. As mentioned earlier, these exponents were obtained by fitting the data on the metallic side only.

Kagawa et al. did the same measurement on $\kappa-(\mathrm{ET})_{2} \mathrm{Cu}\left[\mathrm{N}(\mathrm{CN})_{2}\right] \mathrm{Cl}$ and also extracted critical exponents from the scaled data on the metallic side around the critical end point [KMK05]. Their critical exponents of $(\delta, \beta)=(2,1)$ did not match any known universality class and could be obtained later even by NMR measurements of the same group [KMK09]. To conclude, this interpretation of the Mott transition assumes that the conductivity is only proportional to the order parameter.

Considering the energy density in addition. A different interpretation of the data was given later by Papanikolaou et al., who presented a phenomenological description of the Mott transition, which does not only depend on the thermodynamic order parameter $m$ that is associated with the transition $\left[\mathrm{PFF}^{+} 08\right]$. They assumed an Ising critical point and showed that the conductivity should in addition also depend on the energy density, such that $\Delta \sigma \propto|m|^{\theta}$, where the exponent is a combination of $\alpha$ and $\beta$, namely $\theta=$ $(1-\alpha) / \beta$. With the assumption that $\sigma$ depends on two observables, they could identify a regime where the coupling to the energy density dominates and where the critical exponents $(\delta, \beta)=(15 / 8,1)$ agree within the error bars with those of Kagawa et al.. 
Furthermore, in their description weak disorder crucially influences the size of the critical region: By adding weak disorder the first-order Mott transition gets broadened and the conductance jump is rounded to a continuous transition.

Another scaling theory which aims to also account for results obtained with thermal expansion measurements was given by Bartosch et al. [BdSL10]. Their goal was to explain the divergence of the Grüneisen ratio $\Sigma_{p}$, which is the ratio of thermal expansivity $\alpha_{p}$ and specific heat $c_{p}$, in vicinity of the critical point, which was observed earlier in an experiment by de Souza et al. $\left[\mathrm{dSBS}^{+} 07\right]$. They assumed a general scaling form which includes temperature-like and pressure-like scaling variables and could fit the thermal expansivity data to the prediction of their theory once they assumed critical exponents of 2D Ising universality class.

Including lattice effects via crystal elasticity. In order to explain measurements near the Mott critical point via thermal expansion measurements, it is also possible to include lattice effects explicitly by introducing a coupling of electrons to crystal elasticity in the scaling theory. Zacharias et al. used an effective field theory where they coupled the elastic strain tensor to the Ising order parameter and found a region close to the critical end-point, which is characterized by Landau mean-field behavior [ZBG12, ZRG15]. In the context of ferroelectricity it was already shown that such an isostructural instability can suppress microscopic fluctuations and thereby change the criticality of the end point from Ising to Landau criticality [LS70, Vil70]. For this region, Zacharias et al. predicted a breakdown of Hooke's law of elasticity which results in mean-field exponents. Recently, Gati et al. could verify this claim experimentally by measuring the thermal expansion of $\kappa-(\mathrm{ET})_{2}\left[\mathrm{~N}(\mathrm{CN})_{2}\right] \mathrm{Cl}$ around the Mott critical end point $\left[\mathrm{GGM}^{+} 16\right]$.

The inverse coherence length as a scaling function. Turning back to conductivity measurements on the organic charge-transfer salts, in recent work by Abdel-Jawad et al. $\left[\mathrm{AJKW}^{+} 15\right]$ it was assumed, that the free energy and the correlation function can be described by a single function of the reduced temperature $t=\left(T-T_{c}\right) / T_{c}$ and of the reduced pressure $p=\left(P-P_{c}\right) / P_{c}$, namely the coherence length (in the insulator rather the localization length) denoted by $\xi(t, p)$. According to scaling theory [ $\mathrm{KGH}^{+} 67$, Voj03] they used scaling functions $g_{\xi}^{ \pm}$for $t \gtrless 0$ to express this function as $\xi(t, p)=p^{-\nu_{c}} g_{\xi}^{ \pm}\left(p / t^{\Delta}\right)$, where they used the gap exponent $\Delta=\delta \beta$. As the temperature diverges at $t=0$ along the critical line where $p=0$, the exponent $\nu=\nu_{c} \Delta$ was introduced. The conductivity has been scaled both on the metallic and on the insulating side according to $\left(\sigma^{\text {met }}-\sigma_{c}\right) \propto|p|^{\nu_{c}}$ and $\left(\log \left(\sigma_{c}\right)-\log \left(\sigma^{\text {ins }}\right)\right) \propto|p|^{\nu_{c}}$ respectively. The critical isotherm on the metallic side allowed to extract $\nu_{c}=0.52 \approx 8 / 15$, but on the insulating side two regimes could be identified: Close to the critical point the exponent was identical to the metallic one, but farther off the critical point it was $\nu=1.3$. From the measured Seebeck coefficients $S_{B}$ on the insulating and metallic side of the transition, as well as the conductivity $\sigma$ on the metallic side they could also obtain the gap exponent $\Delta$ by plotting $1 /\left(\xi(t, p) p^{\nu_{c}}\right)$ against $p /|t|^{\Delta}$. They showed, that a scaling with $\Delta=15 / 8$ and $\nu_{c}=0.5333$ could be obtained once they assumed that $S_{B}^{\text {met }}-S_{B}^{\text {crit }} \propto 1 / \xi^{3 / 4}, \sigma_{\text {met }} \propto 1 / \xi$ and $S_{B}^{\text {ins }}-S_{B}^{\text {crit }} \propto 1 / \xi^{1 / 2}$. These exponents are indeed the ones of $2 \mathrm{D}$ Ising universality as $(\delta, \beta)_{\text {Ising }}=(15,1 / 8)$.

The authors also mention, that assigning $\sigma_{\text {met }} \propto 1 / \xi$ and analyzing the data of Kagawa et al. leads to critical exponents of $\nu_{c}=1 / 2$ and $\nu \approx 1$, which are the critical exponents of 2D Ising universality. Doing the same scaling for the chromium-doped vanadium oxide data of Limelette et al. results in critical exponents of mean-field theory. To conclude, 
by assuming the conductivity being proportional to the inverse coherence length $\xi$ measurements on the $\kappa$ - $\mathrm{Cl}$ and $\mathrm{EtMe}_{3} \mathrm{P}\left[\mathrm{Pd}(\mathrm{dmit})_{2}\right]_{2}$ compounds could be reconciled with $2 \mathrm{D}$ Ising universality class.

Marginal quantum criticality. Another possible explanation for unconventional quantum criticality is promoted by $M$. Imada, who introduced the notion of marginal quantum criticality to the Mott transition of organic charge-transfer salts [Ima04, Ima05, MI07, IMY10]. He introduced a phenomenological theory to describe the Mott transition of electronic systems (i.e. without considering additional lattice effects) and shows that in his type of quantum phase transition a smooth transition from Ising-like at finite-temperature to unconventional quantum critical behavior at zero temperatures can be obtained.

Starting point is a parameter that controls quantum fluctuations and causes a topological transition of the Fermi surface from a metal to an insulator at zero temperature. The key idea entering his theory is the idea of multi-furcating or cascading quantum criticality, meaning that the instability of the Mott transition triggers additional instabilities which lead to additional charge, magnetic or superconducting transitions as long as the unstable fixed point of the transition coexists with a quantum degeneracy [Ima04]. In this picture, the critical end point $T_{c}$ of the first-order transition is suppressed and a marginal critical point exists at zero temperature instead, see figure 1.2. One of the results is the formation of inhomogeneous structures on the Fermi surface.
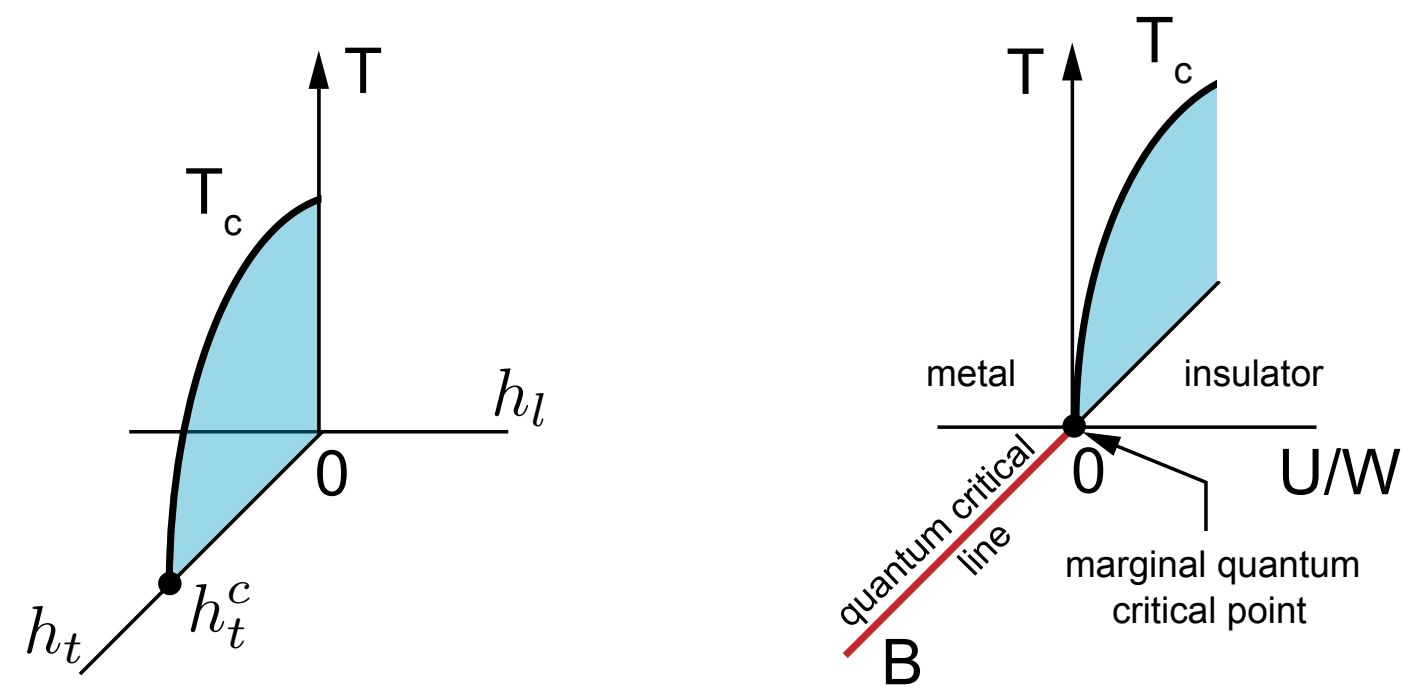

Figure 1.2: Left panel: Schematic phase diagram of the Ising model with a longitudinal $\left(h_{l}\right)$ and a transversal $\left(h_{t}\right)$ magnetic field. The (up- and down-spin) phases are separated by a firstorder transition for $T<T_{c}$ and $h_{t}<h_{t}^{c}$. At the quantum critical point $h_{t}^{c}$ the transition becomes continuous at zero temperature. Right panel: Schematic metal-insulator transition as proposed by Imada. Metal and insulator are separated by a first-order transition, which can be tuned by some control parameter, e.g. $U / W$. Another parameter $B$, which tunes quantum fluctuations, drives the critical temperature $T_{c}$ to zero at a marginal quantum critical point. Larger values of $B$ lead to a quantum critical line, where a continuous topological transition happens at zero temperature. Figures adapted from [Ima04, MI07, IMY10].

Hyperscaling at continuous metal-insulator transitions. In order to describe continuous metal-insulator transitions, the concept of hyperscaling has been introduced in 
the context of localization by disorder [AALR79], as a continuous metal-insulator transition at zero temperature can be obtained by introducing disorder. In general hyperscaling can be thought of being the low-dimensional counterpart of mean-field theories such as the Ginzburg-Landau-Wilson scheme, which are valid above some critical dimension $d_{c}$. This concept can be adopted to continuous metal-insulator transitions which are caused by electron correlations [IFT98] such as the Mott transition. The key assumption behind hyperscaling is that there is only one diverging length scale $\xi$ and a corresponding frequency scale $\Omega$, which are characteristic for the system. Therefore, under the assumption of hyperscaling and at zero temperature, the singular part of the free energy $f_{s}$ can be expressed as

$$
f_{s} \propto \xi^{-(d+z)},
$$

where $d$ denotes the dimension of the system and $z$ is the so-called dynamical exponent. $z$ is defined as the ratio $\ln \xi_{t} / \ln \xi$ when asymptotically approaching the critical point and describes the quantum dynamics of the system. In the metallic phase, $\xi$ is the correlation length and can be defined as $\xi=\sqrt[d]{\int d r\langle X(r, 0) X(0,0)\rangle /\langle X(0,0) X(0,0)\rangle}$, and the temporal correlation length is given by $\xi_{t}=\sqrt{\int d t\langle X(0, t) X(0,0)\rangle /\langle X(0,0) X(0,0)\rangle}$, whereas in the insulating phase $\xi$ denotes the localization length of carriers [MI07]. It can be shown [MI07], that $\xi$ is proportional to the inverse Fermi wave number $k_{F}^{-1} \propto X^{-1 / d}$ for a given carrier concentration $X$. As the gap goes to zero, the divergence of $\xi$ and $\Omega$ can be used to define the critical exponent of the correlation length $\nu$ via $\xi \propto|\Delta|^{\nu}$ and the dynamical exponent via $\Omega \propto|\Delta|^{z \nu}$ [IFT98, Voj03]. In the end, the singular part of the free energy can be expressed as $f_{s}(\Delta) \propto \Delta^{\nu(d+z)}$.

To summarize, experimental studies of the Mott transition on layered organic chargetransfer salts lead to different conclusions with respect to the universality class of the transition. The experiments not only differ in the measured quantities and used techniques, but their measurements also allow for different results with respect to critical exponents as various scaling theories have been motivated and used to derive them. Their findings can be sorted into two groups. One is supporting a 2D Ising universality class $\left[\mathrm{BdSL} 10, \mathrm{AJKW}^{+} 15\right]$ and the other one unconventional critical exponents [KMK05, KMK09, $\mathrm{FMT}^{+}$15], which are connected with quantum critical behavior.

The question of putative quantum critical behavior and the universality class of the transition will be discussed in chapter 4 . There, quantum critical behavior is investigated in a paradigmatic model for the Mott transition, the Hubbard model, and a new route to account for low-temperature critical end points $T_{c}$ is proposed. 


\section{\begin{tabular}{l|l} 
Heavy Fermion Systems & 1.3
\end{tabular}}

The following section addresses some of the known properties of heavy fermion systems [Hew93, Ste14]. Lots of experimental findings for these systems are well described by the paradigmatic Kondo lattice model (KLM), which is investigated in this thesis. The Doniach diagram, which is based on an analysis of this model, is already introduced here. However, more details on the weak and strong coupling limits of the KLM that enter the Doniach diagram are discussed in context of the KLM in section 2.3.

This section is structured as follows. First, the characteristics of heavy fermion systems are introduced and a possible definition of them is given. Afterwards it is briefly explained how one can derive an effective model for these kind of systems. Finally, a focus is set on superconductivity and different mechanisms that were proposed to account for its emergence.

In subsection 1.3.1, conceptional differences between Kondo and Kondo lattice systems are presented. Subsection 1.3.2 introduces the Doniach diagram which describes the interplay of Kondo singlet formation and antiferromagnetism in heavy fermion systems. The topic of quantum critical points (QCP) that are found in some of these systems and the notion of the so-called Kondo breakdown are briefly discussed in this subsection. It will be important for the interpretation of some of the results for the antiferromagnetic phase of the KLM in section 5.2.

"Heavy" fermions. Certain intermetallic compounds that contain elements with partially filled f-electron shells such as $C e, Y b, U, P u$ and $N p$ are called f-electron or heavyfermion systems [Ste14]. Especially those heavy-fermion systems which are based on rare-earth elements will be discussed in the following as they can be described by the Kondo lattice model and are therefore sometimes called dense Kondo systems or Kondo lattices [Hew93].

As the name already suggests, heavy fermion metals have charge carriers with an extremely enlarged effective mass $m^{*}$, which can be hundreds or even over thousand times larger than the bare electron mass. Often, the specific heat coefficients $\gamma$ are used as a criterion to classify a system as a heavy fermion compound: If the coefficient exceeds a somewhat arbitrary threshold value of $400 \mathrm{~mJ} \mathrm{~K} / \mathrm{mol}$ [Hew93], the compound is called a heavy fermion system.

To give a few examples, the first known heavy fermion compound $\mathrm{CeAl}_{3}$ has a specific heat coefficient of $\gamma \sim 1620 \mathrm{~mJ} \mathrm{~K} / \mathrm{mol}$ [AGO75] and the first known superconducting compound $\mathrm{CeCu}_{2} \mathrm{Si}_{2}$ a coefficient of $\gamma \sim 1000 \mathrm{~mJ} \mathrm{~K} / \mathrm{mol}\left[\mathrm{SAB}^{+} 79\right]$. Both of them are based on cerium, but also systems based on other rare-earth elements fulfill this criterion. This includes $\mathrm{YbRh}_{2} \mathrm{Si}_{2}$, a Kondo lattice system which posseses a quantum critical point involving a Kondo breakdown (this will be discussed in section 1.3.2). Even some materials based on actinides, such as $\mathrm{UPt}_{3}(\gamma \sim 420 \mathrm{~mJ} \mathrm{~K} / \mathrm{mol}$ to $450 \mathrm{~mJ} \mathrm{~K} / \mathrm{mol}$, [FFdBM83, SFWS84] $)$ are heavy fermion materials.

This definition of heavy fermion compounds builds on measurements of the specific heat, which would for an usual metal and for temperatures below $T_{\text {Debye }} / 50$ consist of electronic and phononic terms and therefore follow the relation $C_{V} / T=\gamma+\beta T^{2}$. For most heavy fermion systems deviations of this behavior occur, especially for those that are superconducting at low temperature. Sometimes it is sufficient to include additional terms, e.g. a spin-fluctuation term $\sim T^{3} \ln T$ for $U t_{3}$ [SFWS84], but often the systems show anomalies 
at small temperatures, e.g. $\mathrm{CeCu}_{2} \mathrm{Si}_{2}$ [Ste84]. Nevertheless, the intercept in a $C_{V} / T$ vs. $T^{2}$ plot is used to determine the Sommerfeld coefficient $\gamma$ from which a heavily enhanced effective mass $m^{*}$ can be deduced.

Another quantity which is often used as a criterion is the so-called Wilson ratio of susceptibility to Sommerfeld coefficient $R \propto \chi / \gamma$. For an ordinary Fermi liquid this ratio would be one, but in the case of heavy fermions it usually lies between two and five [Ste84]. The magnetic susceptibility that enters this expression usually follows the Curie-Weiss law for high temperatures $(T \sim 100 \mathrm{~K}$ to $300 \mathrm{~K})$, but deviations occur at lower temperature. This is usually attributed to the quenching of the local moments at temperatures smaller than some characteristic Kondo screening temperature $T_{0}$ [Ste84], which is also known as the Kondo effect [Kon64], which is explained in subsection 1.3.1. However, in other heavy fermion compounds the low-temperature anomaly of the magnetic susceptibility was argued to be a consequence of inter-configuration fluctuations between a magnetic and a non-magnetic ground state with the same energy [SV76].

f-electrons and Kondo lattice mapping. In order to construct effective models for these systems, it is important to first of all take a look at the f-shell configuration of the compound. The focus is now set on rare-earth based heavy fermion systems such as $\mathrm{CeCu}_{2} \mathrm{Si}_{2}$ and $\mathrm{YbRh}_{2} \mathrm{Si}_{2}$. CeCu $\mathrm{Si}_{2}$ contains a $\mathrm{Ce}^{3+}$ atom which has the configuration [Xe] $4 \mathrm{f}^{1} 5 \mathrm{~d}^{1} 6 \mathrm{~s}^{2}$ [KYRa15]. Here, the $5 \mathrm{~d}$ and 6 s electrons form the conduction band and in $C e^{3+}$ only one electron is left, namely the $4 \mathrm{f}$ shell electron which is strongly localized at the core. To see which state the remaining f-electron is in, one has to consider the multiplet that emerges due to Hund's rule. The orbital angular momentum quantum number for f-shells is $L=3$ and the spin of the f-electron is $S=1 / 2$, which leads to a multiplet $|1,3,1 / 2\rangle$. However, spin-orbit coupling can split this multiplet into multiplets $|1,3,1 / 2, j\rangle$ where the degeneracy of the new multiplets is given by $N_{j}=2 j+1$, which is in case of the primary multiplet $j=|L-S|=5 / 2$ a multiplicity of $N_{5 / 2}=6$.

In case of $\mathrm{YbRh}_{2} \mathrm{Si}_{2}$ the configuration of Ytterbium reads [Xe] $4 \mathrm{f}^{1} 46 \mathrm{~s}^{2}$ [KYRa15], which means that $Y b^{3+}$ is left with 13 f-electrons - one less than the totally filled 4 f-shell. That means, that for $\mathrm{Ce}^{3+}$ and $\mathrm{Yb}^{3+}$ atoms there is only one electron or one hole in the f-shell. As Hund's rules state that for more than half-filled shells the level with highest value of $j$ is the lowest in energy, the eight-fold degenerate multiplet with $j=7 / 2$ is realized.

Both compounds, $\mathrm{CeCu}_{2} \mathrm{Si}_{2}$ and $\mathrm{YbRh}_{2} \mathrm{Si}_{2}$, form a tetragonal crystal structure which leads to additional Kramers splitting of the degenerate f-electron multiplet due to the tetragonal crystal field. Sticking to the case of $C e$, the ground state of the system at low temperatures corresponds to the electron configuration where the lowest Kramers doublet is filled with the one f-electron of $C e^{3+}$. In this case, the f-shell electron can be mapped to a spin- $1 / 2$ moment coupled to a conduction band, which amounts to the Kondo lattice model. As the f-shell electrons are very localized at the nucleus, they do not participate much in the electronic transport of the conduction band electrons in the s-,p- and dshells. Their magnetic moment is still perceived by the conduction band electrons and influences the ground state properties of the system at low temperatures tremendously. At low energies and due to the strong Coulomb interactions between the f-electrons, they can be mapped to immobile spins, which couple antiferromagnetically and locally to the conduction electrons. The easiest model which captures these physics is called Kondo lattice model and will be introduced in the following section. 
Superconductivity. In 1979, Steglich and co-workers discovered superconductivity for temperatures below $T_{c} \approx 0.6 \mathrm{~K}$ in the heavy fermion compound $C e C u_{2} S i_{2}$ [SAB $\left.{ }^{+} 79\right]$. This was not only the first measurement of heavy fermion superconductivity, but came also as a big surprise as its nature is very unconventional.

Until this discovery, the known superconducting materials were successfully described by the theory of Bardeen, Cooper and Schrieffer (so-called BCS theory) [BCS57]. In those materials it was observed that even small concentrations of magnetic impurities destroy superconductivity, e.g. when doping $G d$ into (superconducting) $L a A l_{2}$ a critical concentration of only 0.59 atomic percent $G d$ is sufficient to destroy superconductivity [Map68]. The reduction of the critical temperature $T_{c}$ with increasing impurity doping is well described by a theoretical prediction of Gor'kov and Abrikosov, who investigated the effect of spin-exchange scattering between the conduction electrons and the $4 \mathrm{f}$-shell electrons of the impurities on superconductivity [AG61]. It was thereby concluded that the magnetic impurities destroy the BCS-like pairing of the conduction electrons.
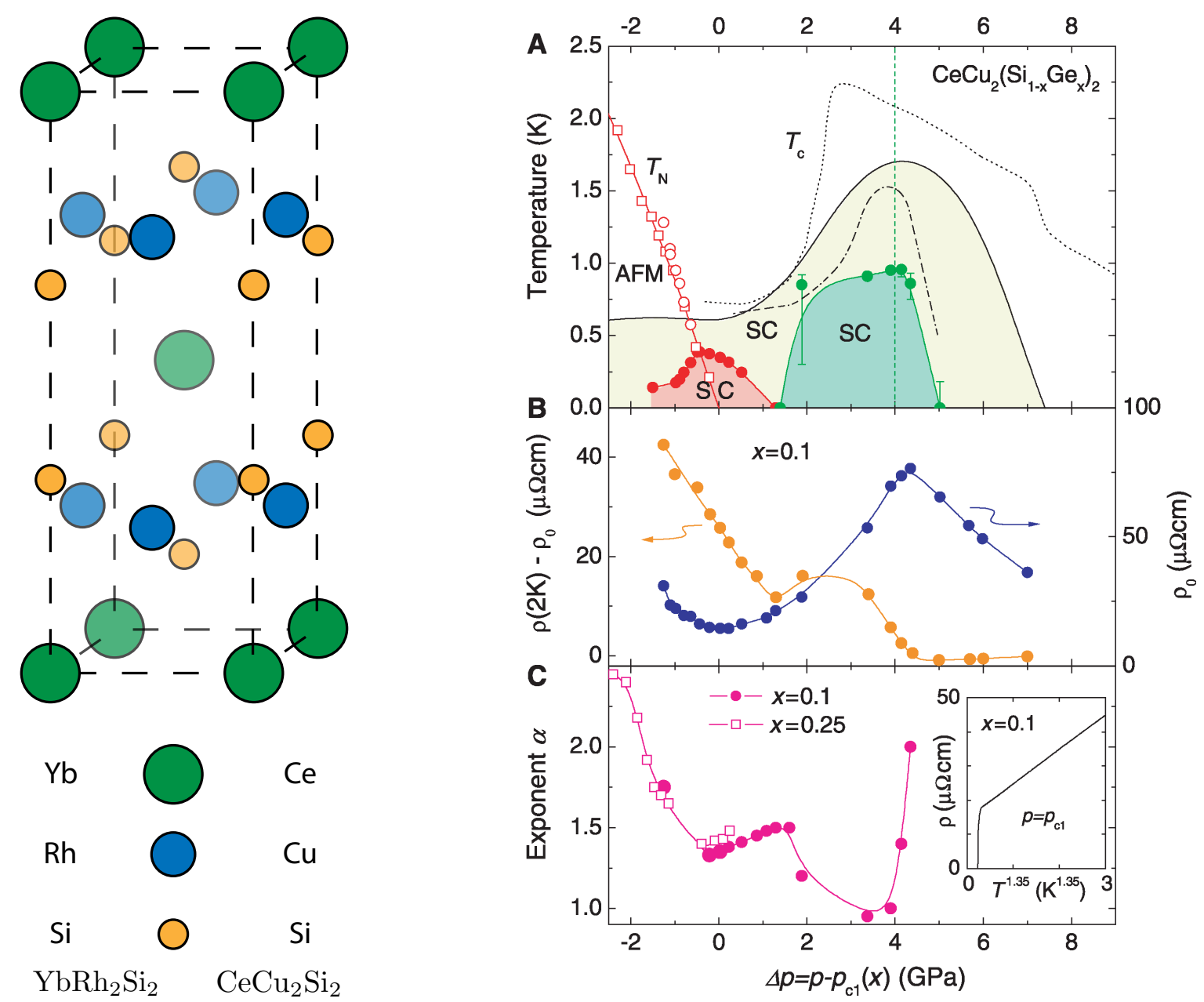

Figure 1.3: Left panel: Tetragonal crystal structure of $\mathrm{CeCu}_{2} \mathrm{Si}_{2}$ and $\mathrm{YbRh}_{2} \mathrm{Si}_{2}$. Top right panel: Phase diagram of 10 at\%-Ge-doped $\mathrm{CeCu}_{2} \mathrm{Si}_{2}$ as a function of temperature and pressure, obtained from experiment. Two distinct superconducting domes (shown in green and red) suggest that more than one pairing mechanism might operate. The broad continuous superconducting region (shown in yellow) amounts to pure $\mathrm{CeCu}_{2} \mathrm{Si}_{2}$. From H. Q. Yuan et al., Science 302, 2104-2107 (2003). Reprinted with permission from AAAS. 
Superconductivity in a system, which contains not only a few atomic percent of magnetic atoms, but shows a periodic lattice of magnetic $\mathrm{Ce}^{3+}$ atoms, was unexpected. In a subsequent study it was shown that this very periodicity of magnetic atoms seems to be the necessary prerequisite to show superconducting behavior: Even small doping of $\mathrm{Ce} \mathrm{Cu}_{2} \mathrm{Si}_{2}$ with non-magnetic atoms or slightly changing the stoichiometry destroys the superconductivity [SRS83] and the isostructural analog with non-magnetic atoms $\mathrm{LaCu}_{2} \mathrm{Si}_{2}$ is not superconducting at all $\left[\mathrm{SAB}^{+} 79\right]$.

The "unconventional" nature of superconductivity consists in the fact that the Cooper pairs are formed by heavy fermion quasiparticles. As a consequence, standard BCS theory for phonon-mediated superconductivity cannot explain this kind of superconductivity. The reason lies in the fact that phonons have a typical frequency which is in the case of $\mathrm{CeCu}_{2} \mathrm{Si}_{2}$ comparable with the fermions' characteristic frequency [SAB $\left.{ }^{+} 79\right]$. Another mechanism has to be used for explanation.

Antiferromagnetic spin-fluctuations were proposed theoretically to act as a glue between the quasiparticles [MSRV86, SLH86] and neutron diffraction experiments suggested the coupling between superconducting and magnetic order parameters in $U P t_{3}\left[\mathrm{ABB}^{+} 89\right]$. Still, the microscopic origin of superconductivity in heavy fermion materials remained an open question and in the meantime different alternative mechanisms have been proposed. It turned out that more than one non-phononic mechanism is needed to discribe different heavy fermion superconductors: In 2001 antiferromagnetic magnons were proposed to lead to an effective interaction between the itinerant electrons and thereby induce superconductivity in $\mathrm{UPd}_{2} \mathrm{Al}_{3}\left[\mathrm{SAM}^{+} 01\right]$ and in 2007 even ferromagnetic spin fluctuations were suggested as a mediator in $U C o G e\left[\mathrm{HGdN}^{+} 07\right]$.

Other mechanisms include charge fluctuations, e.g., in case of the second superconducting phase at high pressure of the Ge-doped prototypical heavy fermion superconductor $\mathrm{CeCu}_{2} \mathrm{Si}_{2} \quad\left[\mathrm{YGD}^{+} 03\right]$, see right panel of figure 1.3. However, the undoped compound $\mathrm{CeCu}_{2} \mathrm{Si}_{2}$ shows superconductivity that is driven by antiferromagnetic excitations $\left[\mathrm{SAF}^{+} 11\right]$. For the $\mathrm{Pr}^{3+}$ moments in $\mathrm{PrOs}_{4} \mathrm{Sb}_{12}$ even quadrupolar fluctuations have been suggested $\left[\mathrm{BFH}^{+} 02\right]$.

Although there are some candidates for triplet superconductivity, e.g. the aforementioned $\mathrm{UPt}_{3}$ [TKI ${ }^{+}$98], most of the heavy fermion superconductors show singlet superconductivity which often turns out to have d-wave symmetry [SW16].

In section 5.4.1, the Kondo lattice model, a paradigmatic model for heavy fermion materials, is probed for this kind of superconductivity by means of the variational cluster approximation. With this technique antiferromagnetic fluctuations and even antiferromagnetic phases can be investigated such that it is even possible to investigate the interplay between superconductivity and antiferromagnetism. The Kondo lattice model is introduced in the chapter on models 2.3, the variational cluster approximation in the chapter on the theoretical framework 3.2.2.

\section{From Kondo to Kondo Lattice Physics $\mid 1.3 .1$}

In this subsection the Kondo effect and the conceptional differences between the Kondo and Kondo lattice model are briefly summarized. A detailed discussion of the history of the Kondo problem and the physics of the Kondo and Kondo lattice model can be found in references [Wil75, Hew93, Col07, Col15a]. 
Historically, before interest in heavy fermion systems awakened, the resistance of metals was studied in detail at low temperatures. In contrast to a drop to zero resistivity in some pure metals, which could be successfully explained by BCS theory of superconductivity [BCS57], a lot of metals showed a finite resistivity for $T \rightarrow 0$. This residual resistivity was soon attributed to electron scattering due to imperfections such as neutral impurities or lattice defects. Some of the metals which were investigated already in the early 1930s instead showed a minimum in resistivity at small temperatures (e.g. $T_{\min } \sim 4 \mathrm{~K}$ for gold) [MV30a, MV30b, dHdBvdB34]. The decrease in resistivity when cooling the system could be understood by considering phonon scattering effects, but the increase of resistivity when approaching zero temperature was unexpected as Matthiessen's rule [MV64] was expected to hold. It states that the resistance is made up of the residual resistivity due to scattering off (neutral) impurities and the ideal resistivity which includes the increase due to phonons.

An explanation for this increase was given in 1964 by Kondo who investigated the scattering of electrons off magnetic impurities [Kon64]. In this paper he investigated the so-called s-d model, which includes an exchange interaction between the spin of the magnetic impurity and the conduction electrons. When considering models to describe this embedding of a magnetic impurity such as the s-d model considered by Kondo, the scattering process between d- or f- electrons of the magnetic impurity and conduction electrons is modeled by an antiferromagnetic exchange term with strength $J$. As already mentioned in the previous section, it is assumed that the d- or f-electrons of the magnetic impurity are strongly localized and only weakly hybridize with the conduction electrons of the metal. Instead, the itinerant electrons only notice the spin of the localized impurity electrons and interact via 'spin-flip' scattering. These exchange interactions which allow for flipping both the spin of the magnetic impurity and the spin of a conduction electron lead to a qualitatively different behavior compared to non-magnetic impurities. Using third-order perturbation theory in the exchange interaction, Kondo showed that this scattering processes lead to a logarithmic correction to the resistivity in the limit $T \rightarrow 0$. Together with the phononic contribution this $\log (T)$ divergence leads to a minimum in resistivity. Despite reproducing the resistance minimum, the perturbative treatment includes a logarithmic divergence which contradicted experimental observations and remained to be a problem for a long time.

Although progress was made in subsequent years it was not before 1974 that a nonperturbative technique, so-called numerical renormalization group, was developed by Wilson, which allowed to solve the Kondo problem [Wil74, Wil75]. The qualitative picture that emerged from solving the Kondo problem is the following. When inserting a magnetic impurity into the sea of conduction electrons, due to the exchange interaction with conduction electrons a spin-singlet between impurity spin and conduction electrons develops. The ground-state is thereby a many-particle state, where conduction electrons screen the local moment of the impurity by forming the singlet. As thermal fluctuations destroy this screening, the singlet forms only below a characteristic temperature, the Kondo temperature $T_{K}$. Right at the Fermi energy the magnetic scattering processes cause a many-body resonance in the spectral density $\rho(\omega)$, the so-called Abrikosov-Suhl or Kondo resonance.

When increasing the amount of magnetic impurities that are alloyed into the metal, one arrives at a dense Kondo system, where at some point a periodic arrangement of mag- 
netic impurities leads to conceptionally different physics. This has to do with an additional mechanism that competes with the Kondo singlet formation. Already when adding a second impurity spin to the conduction electrons, it couples to the spin density around the first impurity. As the first impurity already caused spatially decaying Friedel oscillations, the addition of the second impurity leads to an effective long-range magnetic interaction, so-called RKKY interaction, which will be discussed in more detail in subsection 2.3. Assuming a dense Kondo lattice, where electrons scatter at each lattice site with a magnetic impurity spin, leads to the Kondo lattice model. This model will be discussed in more detail in subsection 2.3. Such a dense Kondo lattice was proposed by Doniach in 1977 to describe heavy-electron metals [Don77]. In this case, a Kondo resonance is created in each unit cell and electrons can scatter off this periodic lattice of resonances. Due to Bloch's theorem this leads to the formation of a heavy fermion band of width $T_{K}$ [Col07, Col15a].

\section{\begin{tabular}{l|l} 
The Doniach Diagram & 1.3 .2
\end{tabular}}

There are two competing mechanisms in the system, namely Kondo spin singlet formation [Kon64] at strong coupling and antiferromagnetic ordering due to effective RKKY interaction at weak coupling. This competition leads to the so-called Doniach diagram [Don77] of the Kondo lattice model. As the emerging picture is often used for the interpretation of experimental data, the Doniach diagram is introduced here. More details about the effective RKKY interaction and the Kondo insulator are discussed in context of the Kondo lattice model in the model section, see subsection 2.3.

In the following the discussion will focus on zero temperature, which is why energy scales will be used instead of temperatures that are usually used when discussing experiments. This is also the notation in which Doniach introduced the concept in 1977 [Don77]. However, the argumentation given below is still valid at low temperatures, i.e. temperatures that are lower than a crossover temperature $T_{0}$ at which incoherent local moments start screening the conduction electrons ("Kondo screening", $T<T_{0}$ ), see figure 1.4. The antiferromagnetic Néel and Fermi liquid phases that emerge then also have a certain temperature $T_{N}$ and $T_{\mathrm{FL}}$ respectively, up to which the argumentation that follows holds.

In the limit of strong coupling, the conduction electrons screen the local moments of the f-electrons and form local Kondo singlets. For weak coupling the local moments induce Friedel oscillations in the spin density of the surrounding conduction electrons. Thereby an effective so-called RKKY interaction [RK54, Kas56, Yos57] is mediated by the conduction electrons and the local moments of the f-electrons order antiferromagnetically. Between the limits of extremely small and strong couplings the Kondo lattice model describes the competition of the local Kondo screening and underscreened RKKY interaction.

In 1977 Doniach analyzed the one-dimensional Kondo lattice model and compared the analytical dependencies of the energies of both limiting states with respect to the coupling $J$ [Don77]. The antiferromagnetically ordered ground state of RKKY-type has an energy

$E_{\mathrm{RKKY}} \propto N_{F} J^{2}$, where $N_{F}$ denotes the electron density at the Fermi energy. For the other state which consists mainly of Kondo singlets the energy goes as $E_{K} \propto \exp \left(-1 / N_{F} J\right)$, which means that both energy scales cross at some critical coupling strength $J_{c}$, see figure 1.4. In his paper, Doniach suggested from these binding energies that the system should undergo a second-order transition from an antiferromagnetic (RKKY) ground state to a 


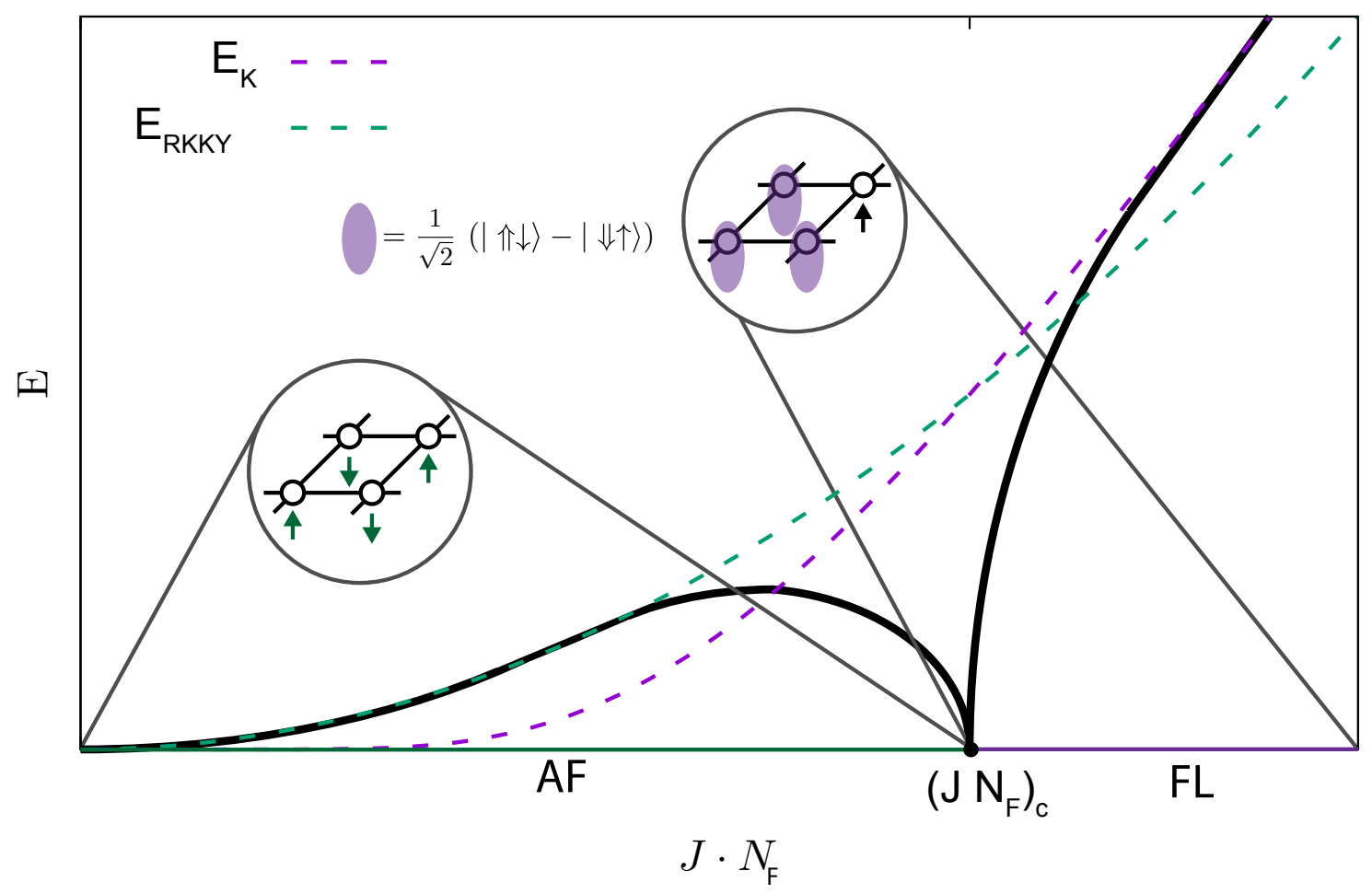

Figure 1.4: Sketch of the Doniach diagram following [Don77], which compares the binding energy of a Kondo singlet $E_{\mathrm{K}} \sim \exp -1 / J N_{F}$ with the energy of a RKKY antiferromagnetic state $E_{\mathrm{RKKY}} \sim J^{2} N_{F}$. At the crossing point, the system is predicted to change from a ground state with RKKY antiferromagnetic ordering of the f-spins to a state with Kondo singlets between fspins and conduction electrons. The solid lines sketch the characteristic energies of the emerging antiferromagnetically ordered and heavy Fermi liquid phases; when translated to temperatures, they correspond to the Néel and Fermi liquid temperature, respectively.

Kondo singlet ground state at $J_{c}$. This is the 'Doniach diagram'. Furthermore, as the exchange coupling $J$ can be tuned in experiment by some non-thermal control parameter like magnetic field or hydrostatic pressure, this transition could (theoretically) be realized at zero temperature which renders the transition point a quantum critical point.

Although Doniach's paper discussed the one-dimensional Kondo lattice model, the proposed continuous phase transition from Kondo insulator to antiferromagnet has been found in the mean-time also for higher dimensional Kondo lattice models. For example Shi et al. used high-order series expansion in the coupling in $d=2$ and $d=3$ and obtained a critical coupling of $J_{c} \approx 1.43$ for the KLM on the square lattice [SSGW95]. Later in 1999, Assaad solved the half-filled two-dimensional KLM numerically with high precision by using quantum Monte Carlo and confirmed the value of $J_{c}=1.45 \pm 0.05$ [Ass99]. A more detailed summary of the numerical techniques that have been used since to investigate the KLM in two dimensions will follow in section 3.3.2.

Kondo breakdown. When comparing the limits of weak and strong coupling away from half-filling, the magnetic order of the state is not the only difference - the Fermi 
surface volume differs strongly, too. In both cases the Luttinger sum rule [Lut60, LW60], which states that the volume of the Fermi surface amounts to the density of electrons, is satisfied. This is quite naturally the case for the small Fermi surface, which is made up by conduction electrons only, but for the heavy Fermi liquid one has to keep in mind, that the number of charge carriers is composed of the conduction electrons and of the f-spins, hence $N=N_{e}+N_{f}$ [Mar82, Osh00]. However, just because the two phases show different Fermi surface sizes, this property does not have to be characteristic for them. That means, that the energy scale $E^{*}$ at which the system changes between a completely Kondo screened phase with a large Fermi surface and an underscreened phase with small Fermi surface does not have to coincide with the transition between antiferromagnet and (paramagnetic) Fermi liquid. This destruction of Kondo singlets at $E^{*}$ goes by the name of Kondo breakdown and is an important criterion to further distinguish two scenarios for quantum criticality that have been heavily discussed in recent years [GSS08, SS10].

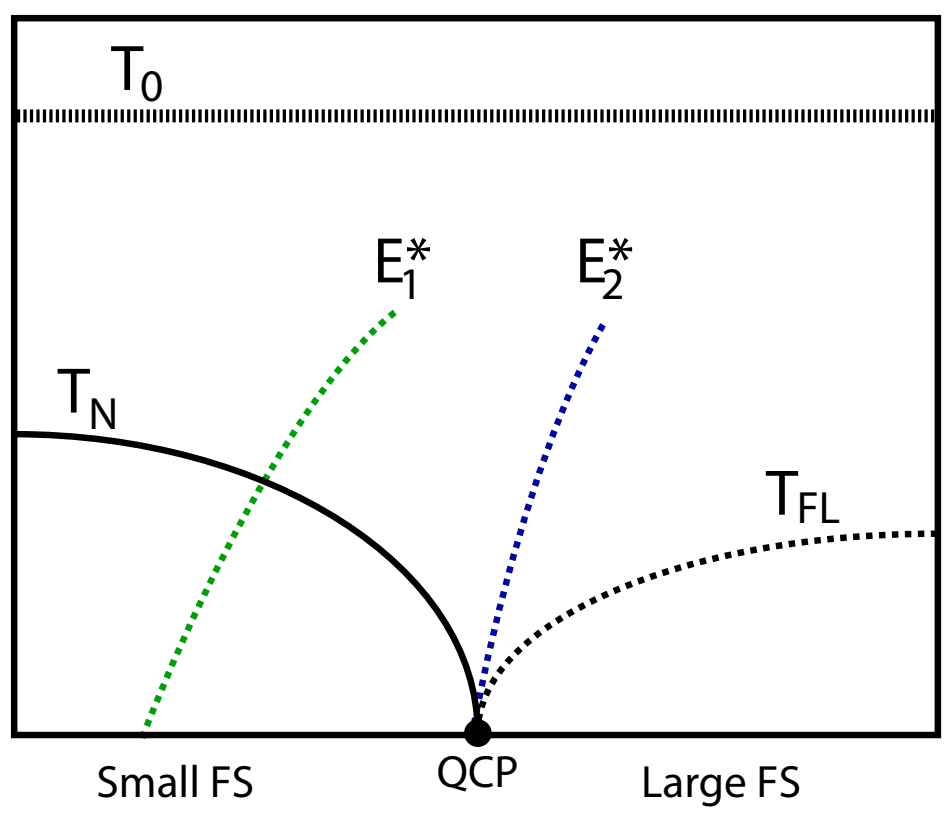

Figure 1.5: Sketch of the two different types of QCPs where $E^{*}$ denotes the Kondo breakdown energy scale. Figure adapted from reference [GSS08].

Quantum critical points. If the breakup of entangled Kondo singlets happens at a coupling strength $J_{*}<J_{c}$ the quantum critical point (QCP) does not coincide with the Kondo breakdown and the QCP is called conventional. The antiferromagnetic phase is thereby splitted into one part with a small Fermi surface and one with large Fermi surface. This QCP has a spin density wave (SDW) form, which means that the antiferromagnetic phase close to the QCP can be described by a spin density wave of heavy quasiparticles in the paramagnetic phase [Ove59, GSS08].

In the other case, the so-called unconventional QCP, the Kondo breakdown coincides with the transition to the antiferromagnetic phase. From conductivity measurements on $\mathrm{YbRh}_{2} \mathrm{Si}_{2}, \Delta \rho(T)=\left|\rho(T)-\rho_{0}\right| \sim T^{\epsilon}$, the exponent $\epsilon$ is extracted and shows a magnetic field-induced quantum phase transition $\left[\mathrm{CGW}^{+} 03\right.$, GSS08, SS10]. At weak coupling, the 
local moments order antiferromagnetically and do not form singlets with the conduction band electrons. As the f electrons are localized and not form Kondo resonances, they do not screen the electrons statically and the Fermi surface is small throughout the entire antiferromagnetic phase. Right at the quantum critical point the Fermi surface volume shows an abrupt jump, as for couplings $J>J_{c}$ the charge carriers immediately include f-moments [SRIS01, CPSR01]. This situation, which is sometimes also referred to as local criticality [SRIS01, GSS08], is, e.g. realized in $\mathrm{CeCu}_{2} \mathrm{Si}_{2}$. In $\mathrm{YbRh}_{2} \mathrm{Si}_{2}$ it has been shown, that applying chemical pressure to the system results in moving the Kondo breakdown into either the antiferromagnetic or paramagnetic phase $\left[\mathrm{FWB}^{+} 09\right]$. Both scenarios are sketched in Figure 1.5.

However, it should be mentioned that recent numerical studies found no Kondo breakdown at any finite coupling strength $J$, although the Fermi surface changed in the antiferromagnetic phase from an electron- to a hole-Fermi surface. This will be discussed in section 3.3.2.

A paradigmatic model for heavy fermion systems, the Kondo lattice model, is investigated in chapter 5 and questions raised in this introductory section are addressed. In particular, the quantum critical point between a metallic phase of heavy charge carriers and an antiferromagnetically ordered phase, as well as the question, whether it coincides with a Kondo breakdown, are discussed. Moreover, the emergence of superconductivity and its interplay with antiferromagnetism is studied and compared to results of other numerical studies on this model; a survey of other numerical approaches to this model follows in section 3.3.2. Finally, the relevance of these findings on the Kondo lattice model for future studies that can be connected closer to experiments are discussed. 


\section{Outline of the Thesis $\mid 1.4$}

\section{Chapter 2: Models}

The models that are treated within this thesis are introduced and some of their properties are discussed. A focus is set on results obtained by other numerical methods.

\section{Chapter 3: Theoretical Framework}

Different Green functions and their numerical calculation are considered. A simple cluster method, so-called cluster perturbation theory is introduced. In an excursus, a spin formulation of this technique using superclusters is derived and its quality is tested.

Then, the self-energy functional theory is introduced. One of the approximations which are based on it, the variational cluster approximation (VCA), is discussed and details of the numerical evaluation are explained. Finally, it is shown how the variational cluster approximation can be applied directly to the Kondo lattice model.

\section{Chapter 4: Mott Quantum Criticality in Two Dimensions}

The strongly anisotropic, frustrated two-dimensional Hubbard model is investigated by means of different flavors of variational cluster approximation.

The Mott transition is investigated as a function of the interchain hopping, which acts as a control parameter driving the transition. A detailed analysis and discussion of the results using variational cluster approximation is complemented by a comparison with finite-temperature results using cellular dynamical mean-field theory (CDMFT) obtained by $M$. Raczkowski. In the discussion of a quantum critical line emerging in the model the question of quantum criticality in Mott systems is approached. The question of the transition's universality class is addressed as far as possible within a technique that is mean-field-like on large distances. Afterwards, the antiferromagnetic phase of the model is investigated. The results are discussed and future research in the context of Imada's marginal quantum criticality is motivated.

\section{Chapter 5: Unconventional Phases in the Kondo Lattice Model}

The Kondo lattice model is investigated by variational cluster approximation.

First, the paramagnetic phase of the model is analyzed and the Kondo insulator at halffilling and a metal with Kondo singlets off half-filling are discussed. Motivated by the Doniach diagram, antiferromagnetic phases at weak coupling are analyzed via VCA in section 5.2. A rough finite-size extrapolation is done at half-filling and two antiferromagnetic metals with different Fermi surface topology are investigated in the doped system. The questions of the existence of a Kondo breakdown and of the type of quantum critical point will be touched upon briefly.

Motivated by the work of Bodensiek et al. the model is probed for s-wave superconductivity. Stationary points are initially identified in a large variational space using a small plaquette and then analyzed with a larger cluster. d-wave superconductivity as it is found experimentally in many Kondo lattice systems is studied afterwards. A stable superconducting phase is analyzed and its interplay with antiferromagnetism is discussed.

\section{Chapter 6: Conclusions}

Finally the results are summarized and perspectives for further studies are given. 



\section{\begin{tabular}{l|l} 
& \\
Models & 2
\end{tabular}}

In the following the models that will be used in subsequent chapters are presented.

First, the one-band Hubbard model and its anisotropic frustrated two-dimensional version, which is investigated in chapter 4, are introduced in subsection 2.1. Limits, in which the model can be solved exactly, are discussed. In the limit of strong interaction, $U / t \gg 1$, the half-filled Hubbard model is equivalent to the Heisenberg model; it is briefly introduced in section 2.2 .

Section 2.3 discusses the Kondo lattice model (KLM) and its limits of weak and strong coupling are revisited.

\section{Investigating the Mott Transition: The Hubbard Model

One of the conceptionally simplest models that describes interacting electrons on a static lattice is the Hubbard model [Hub63, Geb03]. It was introduced as a model for electrons in narrow energy bands, such as $d$ - and $f$-shell electrons [Hub63], and is still one of the main models to study the Mott transition [IFT98]. Two key assumptions are made in order to derive the Hubbard model, namely the assumption of a single band of electrons and local electron-electron interactions [Hub63]. Although the Hubbard model has a seemingly simple appearance, it is only exactly solvable in one $\left[\mathrm{EFK}^{+} 05\right]$ and in infinite dimensions [Geb03]. For the two-dimensional model, which is studied in this thesis, only limiting cases are known exactly and for most investigations approximate methods have to be used.

Here, only some aspects of the Hubbard model are briefly mentioned and the focus is set on the Mott transition. Detailed reviews can be found for example in references [Geb03, IFT98].

The one-band Hubbard model is defined by the following Hamiltonian:

$$
\mathcal{H}=\sum_{i, j} t_{i j}\left(c_{i}^{\dagger} c_{j}+\text { h.c. }\right)+U \sum_{i} n_{i \uparrow} n_{i \downarrow}-\mu \sum_{i} n_{i} .
$$

$U$ denotes the strength of the local Coulomb repulsion term and $\mu$ is the chemical potential by which the filling is set in the grand canonical formulation. Throughout this thesis the Hubbard model will be treated at half-filling $(n=1)$ only. $n_{i \sigma}$ denotes the density operator for electrons of spin $\sigma \in\{\uparrow, \downarrow\}$ at site $i, n_{i}=c_{i \sigma}^{\dagger} c_{i \sigma}$.

The dimensionality and geometry of the lattice enters the model via the hopping matrix $t_{i j}$ and influences the bandwidth $W$ of the non-interacting part of the model. In case of the intensively studied isotropic Hubbard model on a square lattice, the hopping matrix $t_{i j}$ is build up from hopping terms of strength $t$ between nearest neighbor sites, see figure 2.1. The Pauli principle forbids two electrons with the same spin $\sigma$ to occupy the same site, which is why each site can be maximally doubly occupied. 

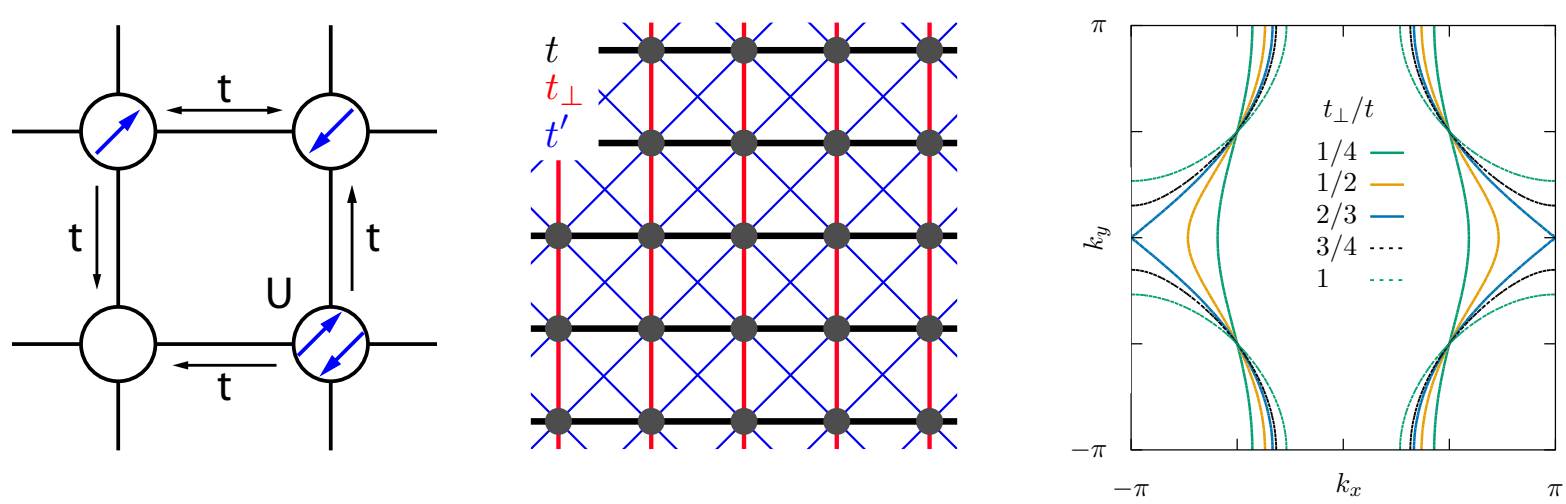

Figure 2.1: Sketch of the isotropic 2D Hubbard model on a square lattice (left panel) and the structure of the hopping terms that enter the anisotropic frustrated 2D Hubbard model considered in chapter 4 (center panel). The right panel shows the Fermi surface of the anisotropic model for $U / t=0$ and $t^{\prime}=-0.25 t_{\perp}$ for different values of $t_{\perp}$.

The model that is investigated in chapter 4 contains anisotropic hopping terms on the square lattice: In x-direction the hopping strength is $t$ ('intrachain hopping'), but in $\mathrm{y}$-direction it is $t_{\perp}$ ('interchain hopping'). The values of the interchain hopping $t_{\perp}$ will range from 0 to $t$. In case of $t_{\perp}=0$ the one-dimensional Hubbard model is recovered and for $t_{\perp} / t=1$ the model amounts to the isotropic two-dimensional (2D) Hubbard model. In addition the model contains next-nearest neighbor hopping with hopping strength $t^{\prime}$, which in a rectangular geometry amounts to diagonal hopping terms, see figure 2.1.

Limit $U=0$. In the limit $U=0$, only the kinetic term of the model remains and the model is solved by Fourier transformation into momentum space. The dispersion of the anisotropic frustrated model then reads $\epsilon\left(k_{x}, k_{y}\right)=-2\left(t \cos \left(k_{x}\right)+t_{\perp} \cos \left(k_{y}\right)\right)-$ $4 t^{\prime} \cos \left(k_{x}\right) \cos \left(k_{y}\right)$. In this limit, the model consists of free fermions on a lattice and therefore describes an ideal metal.

Limit $t=0$. The limit $t=0$ is also known as atomic limit. As no hopping and hence no communication between the sites is allowed, the system consists of empty and single occupied sites for $n<1$ and of single and double occupied sites for $n>1$. At zero temperature the ground state is degenerate and has the minimal number of double occupancies. Due to the absence of any mobility, the system is a (trivial) insulator.

Limit of large interaction. Adding a finite hopping strength to the model in the atomic limit allows one to use perturbation theory in $t$, but even in the limit $U / t \rightarrow$ $\infty$ the model is only solvable in special situations. The ground state is still given by states with lowest possible double occupancy and one can restrict the discussion to this subspace as done, e.g. by the Gutzwiller projection technique [Geb03]. At half-filling and zero temperature, each site is occupied with exactly one electron in the ground state. Excitations of this ground state in form of adding or removing an electron cost energy the system is gapped and hence an insulator.

For large interaction strengths $U / t \gg 1$, one can apply second order perturbation theory 
in the subspace of no doubly occupied sites. Assuming half-filling, the model is thereby found to be equivalent to the Heisenberg model with antiferromagnetic exchange coupling [Geb03]. This model is introduced in subsection 2.2. Within this description the ground state turns out to be an antiferromagnetic insulator.

Metal-Insulator Transition. In the limit $U=0$, the system is metallic and in the limit $U / t \gg 1$ it is insulating. For coupling strengths of the order of the bandwidth $W$ of the non-interacting system, the model is called strongly correlated. It describes competition between itinerant electrons for small $U$ and localization of electrons for large $U$. The Hubbard model is therefore a prototypic model to study Mott metal-insulator transitions (MIT). When considering a frustrated isotropic two-dimensional Hubbard model, the MIT takes place at a finite value $U_{c} / t\left[\mathrm{SGR}^{+} 15\right]$. However, two different insulating phases have to be distinguished. In both cases, the insulator is a many-body effect - localized moments emerge due to electron-electron interactions $U$.

An antiferromagnetic insulator is obtained in the $U / t \gg 1$ limit of the isotropic model, where a gap arises due to long-range antiferromagnetic ordering of preformed moments. In the case of an paramagnetic insulator the preformed moments do not show this longrange order. To differentiate between these two insulators, they are sometimes called Mott-Heisenberg and Mott-Hubbard insulator, respectively [Geb03]. Transitions to both of these insulators are investigated in this thesis.

Other types of insulators that are based on a one-body picture such as Anderson, Band or Peierls insulators as well as the Slater insulator, where the exchange part of the electronelectron interaction is taken into account via a Hartree-Fock description, are not analyzed here.

In order to distinguish metal and insulator at zero temperature, one often takes the existence or absence of a Drude peak as the criterion. For an insulator, the static electrical conductivity $\sigma_{\alpha \beta}=\lim _{\omega \rightarrow 0} \lim _{|\mathbf{k}| \rightarrow 0} \operatorname{Re}\left(\sigma_{\alpha \beta}(\mathbf{k}, \omega)\right)$ vanishes, whereas a metal is expected to have finite Drude weight. Another quantity that can be considered is the charge compressibility, $\kappa=-\partial n / \partial \mu$. For an insulator the compressibility is zero, for a metal it is finite. However, for finite temperatures, due to thermal broadening, a meaningful and precise definition of insulator and metal is barely possible [Geb03].

Exact solutions in $D=1$ and $D=\infty$. In one and infinite dimensions, the Hubbard model can be solved exactly.

For the one-dimensional model, the Bethe ansatz can be used and due to Umklapp scattering processes the system is insulating for any finite Coulomb interaction strength $U$ $\left[\mathrm{EFK}^{+} 05\right]$. Therefore, the metallic phase is limited to $U=0$, which is a difference to the two-dimensional case studied here. However, the anisotropic model, which is studied in this thesis, has the one-dimensional model as a limiting case. The technique that will be used to investigate the model is capable of reproducing this transition and also its qualitative results compare well with the exact Bethe ansatz solution [BHP08].

In case of infinite dimensions, the Hubbard model can be solved numerically using the dynamical mean-field theory [MV89]. As spatial fluctuations are negligible in infinite dimensions, the self-energy becomes a local quantity and the model can be mapped to the single-impurity Anderson model [JP93]. Within DMFT, the MIT is described by the so-called preformed-gap scenario, where the transition happens at a finite critical interaction strength $U_{c}$ : For very small $U$ the density of states form a continuum with two Hubbard bands and a quasi-particle (qp) resonance at the Fermi energy. When increasing 
the interaction strength, the lower and upper Hubbard band move apart, but the qp-peak stays at the Fermi energy. Only its weight changes and vanishes at the critical point $U_{c}$. At zero temperature, the transition is of first order. When increasing the temperature the transition becomes continuous at a critical temperature $T_{c}$.

The effect of geometrical frustration. The diagonal hopping terms of the model considered in this thesis induce frustration to the system. Without these terms, the isotropic system $\left(t_{\perp}=t\right)$ would otherwise order antiferromagnetically and form an antiferromagnetic insulator $\left[\mathrm{SGR}^{+} 15\right]$. They remove the antiferromagnetic perfect nesting instability in the isotropic 2D case [HV98] which can be seen from the Fermi surface of the tight-binding dispersion $(U / t=0)$ in figure 2.1 .

Already a small value of $t^{\prime} / t$ leads to a warping of the Fermi surface and thereby destroys the diamond shape which hosts the antiferromagnetic nesting vector $\mathbf{Q}=(\pi, \pi)$ for $t_{\perp}=t$. In addition, it also removes the Van Hove singularities at the zone boundaries (at $( \pm \pi, 0)$ and $(0, \pm \pi))$ for $t_{\perp} / t=1$. However, for a smaller inter-chain hopping $t_{\perp}^{*} / t$ (see $t_{\perp} / t=2 / 3$ in figure 2.1) one of the singularities still exists at $( \pm \pi, 0)$.

The quasi-1D and quasi-2D regimes. The value of $t_{\perp}^{*} / t$ could be taken as the turning point between a quasi-one dimensional system with a small Fermi surface and a quasi-two dimensional system with a large Fermi surface: For small $t_{\perp} / t$ the Fermi surface resembles the Fermi surface of the one dimensional system of decoupled chains. It gets warped for increasing inter-chain hopping and as a consequence the Fermi surface grows. At $t_{\perp}^{*}$ the warped Fermi surface hits the Brillouin zone boundary at $k_{y}=0$ and for even stronger inter-chain hopping it more and more resembles the one of the isotropic two dimensional system.

Although this considerations for the non-interacting system seem to provide a clear criterion for distinguishing quasi-1D from quasi-2D behavior, it is questionable whether this picture can be transferred to interacting systems. At least for the isotropic system $\left(t_{\perp} / t=1\right)$ several numerical techniques, such as path-integral renormalization group (PIRG) [MI06] and cellular DMFT [KT06], have shown that the antiferromagnetic insulator can be suppressed with a sufficiently large diagonal hopping $t^{\prime} / t$ to move the metal-insulator transition to finite $U / t$. Under the assumption that the system stays metallic for small interaction strengths $U / t$ for all inter-chain hoppings $0 \leq t_{\perp} / t \leq 1$ it should be possible to investigate the Fermi surface and to look for Van Hove singularities. As long as Fermi liquid theory can be applied, the Fermi surface could keep up the $t_{\perp}^{*}$ criterion. Still, it is far from clear how $t_{\perp}^{*}$ changes as a function of interaction strength and how one should define $t_{\perp}^{*}$ in the case of a partially gapped Fermi surface. Especially close to the metal-insulator transition, it has to be expected that the interactions lead to important changes of the Fermi surface, which might ask for a different indication for distinguishing a quasi-1D from a quasi-2D regime. 


\section{Studying Quantum Magnetism: The Heisenberg Model

The 1D spin-1/2 Heisenberg model can be obtained in second order perturbation theory from the $U \rightarrow \infty$ limit of the half-filled Hubbard model [Geb03] and its Hamiltonian reads:

$$
\mathcal{H}=-\sum_{\langle i, j\rangle} J_{i j} \mathbf{S}_{i} \cdot \mathbf{S}_{j}+h \sum_{i} S_{i}^{z}
$$

A magnetic field in $z$-direction with field strength $h$ has been added in the last term. In the following, only positive values of $J_{i j}$ will be used which correspond to an antiferromagnetic interaction. For convenience, the spin operator $\mathbf{S}=\left(S^{x}, S^{y}, S^{z}\right)$ is rewritten in terms of $S^{ \pm}=S^{x} \pm i S^{y}$, which changes the first term of the Hamiltonian to

$$
-\sum_{\langle i, j\rangle} J_{i j}\left(\frac{1}{2}\left(S_{i}^{+} S_{j}^{-}+S_{i}^{-} S_{j}^{+}\right)+S_{i}^{z} S_{j}^{z}\right) .
$$

The commutation relations $\left[S_{i}^{x}, S_{j}^{y}\right]=i \hbar \delta_{i j} S_{i}^{z}$ and cyclic permutations lead to $\left[S_{i}^{+}, S_{j}^{-}\right]=$ $2 \hbar \delta_{i j} S_{i}^{z}$ and $\left[S_{i}^{z}, S_{j}^{ \pm}\right]= \pm \hbar \delta_{i j} S_{i}^{ \pm}$.

The isotropic case corresponds to $J_{i j}=J \delta_{i, i+1}$. By introducing an anisotropy in the exchange interaction with respect to z-direction one arrives at the so-called XXZ Heisenberg chain, defined by

$$
\mathcal{H}_{\mathrm{XXZ}}=-\sum_{\langle i, j\rangle}\left\{\frac{J}{2}\left(S_{i}^{+} S_{j}^{-}+S_{i}^{-} S_{j}^{+}\right)+\Delta S_{i}^{z} S_{j}^{z}\right\}+h \sum_{i} S_{i}^{z} .
$$

Setting $\Delta=J$ leads back to the isotropic model, which is also known as XXX Heisenberg chain. In the following, $\hbar=1$ and everything will be expressed in units in which $J=1$.

The lowest excitations at zero temperature are triplets with total spin $S=1$ and des Cloizeaux and Pearson derived the lower bound of these excitations to be $\epsilon_{1}(k)=\frac{\pi}{2} J|\sin k|$ for an infinite chain without applied magnetic field [dCP62]. For higher energies a twoparameter continuum of spin-wave-type excitations emerges up to a higher bound of $\epsilon_{2}(k)=\pi J|\sin k / 2|$ [MTBB81]. Müller et al. used the analytical Bethe ansatz to extend their calculations and provided analytical and numerical results for the isotropic Heisenberg chain with and without external magnetic field [MTBB81]. In 2012 Caux et al. derived an exact expression for the two-spinon form factor in the XXZ Heisenberg chain [CKSW12], which makes up most of the form factor (in the isotropic case it was shown by Klauser et al. that 4 -spinon and higher excited states contribute only $6 \%$ to the dynamical spin structure factor [KMCvdB11]). Therefore, it is possible to compare the spectra obtained by approximate methods like SCPT with exact results both for the isotropic and the anisotropic Heisenberg chain.

Before turning to the derivation of spin cluster perturbation theory in the next subsection, it is briefly shown that the problems that occur for cluster techniques are at least in parts due to non-local interactions. This can be shown by making a Jordan-Wigner transformation

$$
S^{+} \rightarrow a^{\dagger}, \quad S^{-} \rightarrow a, \quad S^{z} \rightarrow a^{\dagger} a-\frac{1}{2}
$$


where $a^{\dagger}$ and $a$ denote the creation and annihilation operators of spinless fermions. One is left with fermions with standard anti-commutation rules for which "usual" cluster techniques for electrons should be in principle applicable. One obtains the following Hamiltonian:

$$
\mathcal{H}=\sum_{i}\left\{\frac{J}{2}\left(a_{i}^{+} a_{i+1}+a_{i} a_{i+1}^{+}\right)+\Delta\left(n_{i}-\frac{1}{2}\right)\left(n_{i+1}-\frac{1}{2}\right)\right\}+h \sum_{i}\left(n_{i}-\frac{1}{2}\right),
$$

with $n_{i}=a_{i}^{\dagger} a_{i}$. However, the second term poses a problem for cluster techniques, such as $\mathrm{CPT}$ or VCA, as the interactions in the chain of spinless fermions are between neighbors and hence still non-local.

\section{Effective Description of Heavy Fermions: The Kondo Lattice Model}

The Hamiltonian of the Kondo lattice model [Kas56], which is one of the easiest models to describe heavy fermion physics [Hew93], consists of three terms,

$$
\mathcal{H}=-t \sum_{\langle i, j\rangle \sigma}\left(c_{i \sigma}^{\dagger} c_{j \sigma}+\text { h.c. }\right)-\mu \sum_{j} n_{j}+J \sum_{j} \mathbf{S}_{j} \cdot \mathbf{s}_{j} .
$$

The first two describe a spinfull tight binding band with nearest neighbor hopping terms $t$ and a chemical potential $\mu$ by which the filling can be set in a grand canonical formulation. On each site of the lattice, there exists exactly one localized spin (motivated by the localized f-electrons of heavy fermion systems) in addition to the electrons of the tight binding band, see figure 2.2. Here, the Kondo lattice model is studied on a two-dimensional square lattice. This can be motivated from heavy Fermion compounds like $\mathrm{CeCu}_{2} \mathrm{Si}_{2}$, where the atoms responsible for the f-moments (here cerium) build two-dimensional layers in the tetragonal lattice structure, see left panel of figure 1.3. The last term of the Hamiltonian describes the antiferromagnetic spin-spin Heisenberg interaction between these localized spins (also called $f$-spins in the following) and the electrons (also called $c$-electrons in the following as they are motivated by the conduction band electrons of heavy fermion systems). One can rewrite the last term using creation and annihilation operators for the conduction electrons. This is done via $\mathbf{s}_{j}=\left(s_{j}^{x}, s_{j}^{y}, s_{j}^{z}\right)=\frac{1}{2}\left(c_{j \beta}^{\dagger} \sigma_{\beta \alpha} c_{j \alpha}\right)$, where $\sigma$ is the vector of Pauli matrices. Using ladder operators $S^{ \pm}=S^{x} \pm i S^{y}$ for the localized spins $\mathbf{S}_{j}=\left(S_{j}^{x}, S_{j}^{y}, S_{j}^{z}\right)$ leads to:

$$
\begin{aligned}
J \sum_{j} \mathbf{S}_{j} \cdot \mathbf{s}_{j} & =\frac{J}{2} \sum_{j}\left(S_{j}^{x}\left(c_{j \uparrow}^{\dagger} c_{j \downarrow}+c_{j \downarrow}^{\dagger} c_{j \uparrow}\right)+i S_{j}^{y}\left(c_{j \downarrow}^{\dagger} c_{j \uparrow}-c_{j \uparrow}^{\dagger} c_{j \downarrow}\right)+S_{j}^{z}\left(n_{i \uparrow}-n_{i \downarrow}\right)\right) \\
& =\frac{J}{2} \sum_{j}\left(S_{j}^{+} c_{j \downarrow}^{\dagger} c_{j \uparrow}+S_{j}^{-} c_{j \uparrow}^{\dagger} c_{j \downarrow}+S_{j}^{z}\left(n_{i \uparrow}-n_{i \downarrow}\right)\right) .
\end{aligned}
$$

One has to note, that an additional factor $1 / 2$ is "hidden" in the z-component of the spin operator that is acting on the localized spins:

$$
S^{+}|\Downarrow\rangle=|\Uparrow\rangle, \quad S^{-}|\Uparrow\rangle=|\Downarrow\rangle, \quad S^{z}|\Downarrow\rangle=-\frac{1}{2}|\Downarrow\rangle, \quad S^{z}|\Uparrow\rangle=\frac{1}{2}|\Uparrow\rangle .
$$

In this form, the Hamiltonian will be used later to calculate Green functions within the formalism of the variational cluster approximation.

The limits of weak and strong interaction are now discussed in a bit more detail. 


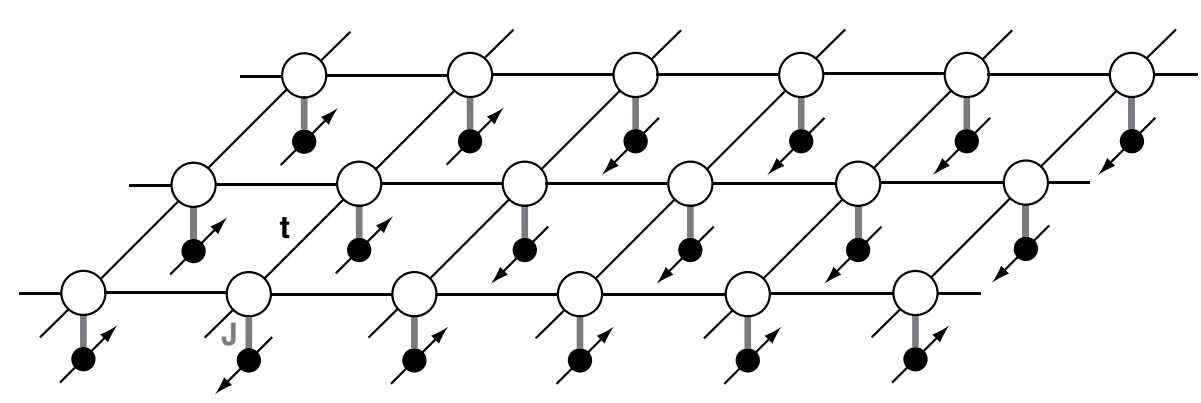

Figure 2.2: The single-band Kondo lattice model on a two-dimensional square lattice.

The Kondo insulator. When considering the strong coupling limit $J \gg t$ of the model, the system consists of local Kondo singlets. The hopping $t$ can then be considered as a small parameter and Heisenberg physics dominates:

$$
\mathcal{H} \stackrel{J \gg t}{\longrightarrow} J \sum_{j} \mathbf{S}_{j} \cdot \mathbf{s}_{j}+\mathcal{O}(t) .
$$

Considering the half-filled case first, one has three triplet states with energy $1 / 4 J$ and a singlet state with energy $-3 / 4 J$ per site. Therefore, the ground state of the system consists of singlets between local spins and conduction electrons. It is a Kondo insulator with the ground state wave function $|\mathrm{GS}\rangle=\prod_{i} 1 / \sqrt{2}\left(|\Uparrow \downarrow\rangle_{i}-|\Downarrow \uparrow\rangle_{i}\right)$. Flipping a spin breaks one of these singlets and turns it into a triplet, which comes with an energy cost of $\Delta E=E(\mid$ triplet $\rangle)-E(\mid$ singlet $\rangle)=J$. Excitations of the ground state $\left|\operatorname{exc}_{i \sigma}^{ \pm}\right\rangle$can be obtained by removing or adding a conduction electron:

Removing a conduction electron destroys one of the singlets, each of which has an energy of $-3 / 4 J$, and leaves an unpaired local spin. Therefore, $\left|\operatorname{exc}_{i^{\prime} \sigma}^{+}\right\rangle=\sqrt{2} c_{\sigma i^{\prime}}|G S\rangle$ has an energy of $3 / 4 J$.

The same holds for adding a conduction electron. It also destroys one of the singlets, but this time an unpaired local spin and a doubly occupied site are left. The energy of $\left|\operatorname{exc}_{i^{\prime} \sigma}^{-}\right\rangle=\sqrt{2} c_{\sigma i}^{\dagger}|G S\rangle$ is therefore $3 / 4 J$, too.

Adding even an infinitesimally small hopping $t$ delocalizes the vacant or doubly occupied site, which can be seen by calculating $\left\langle\operatorname{exc}_{i \sigma}^{-}|\mathcal{H}| \operatorname{exc}_{j \sigma}^{-}\right\rangle=-t / 2+3 / 4 J$. Analogously, the energy of the other excitation $\left|\mathrm{exc}^{+}\right\rangle$is $t / 2+3 / 4 J$. To compare both quasiparticle dispersions, a particle-hole transformation has to be done on one of the excitations. Thereby the dispersion of the first excitation changes to $t_{2}-3 / 4 J$. Afterwards, both dispersions can be compared, such that one ends up with a gap of $3 / 2 J$. A more detailed discussion of the Kondo insulator can be found in [Col15b].

Heavy Fermi liquid. When removing or adding an electron, the finite value of the electron hopping parameter $t$ leads to mobile charge carriers and one obtains a dispersion relation for the doped case. Still, in the case of small doping and strong coupling $J$ instead of considering the movement of unpaired electrons one could also look at the Kondo singlets and observe the mobility of these Kondo singlets. Taking this point of view, the localized spins, although being immobile, participate in transport processes as they form the singlets and are hence part of the charge carriers. They contribute to the Fermi surface and thereby lead to a large Fermi surface, which corresponds to a heavy Fermi liquid that is made out of Kondo singlets. 
RKKY interaction and magnetic long-range order. The considerations so far assumed strong coupling. In the other limit of weak coupling $J / t \ll 1$, Ruderman, Kittel [RK54], Kasuya [Kas56] and Yosida [Yos57] have shown that an effective interaction between neighboring f-spins leads to magnetic long-range ordering. This so-called RKKY interaction is mediated by the conduction band electrons and results in an antiferromagnetically ordered ground state.

To see this, one can first consider a single magnetic moment at site $i$. It induces Friedel oscillations in the spin density of the conduction band electrons: $\langle\mathbf{s}(\mathbf{r})\rangle=-J \chi\left(\mathbf{r}-\mathbf{r}_{i}\right)\left\langle\mathbf{S}_{i}\right\rangle$. Here, $\chi$ is the magnetic susceptibility of the conduction electrons. It can be shown [Col07], that these oscillations decay as $\langle\mathbf{s}(r)\rangle \sim-J \rho \cos \left(2 k_{F} r\right) /\left|k_{F} r\right|^{3}$, where $r$ denotes the distance from the magnetic moment and $\rho$ is the density of states of conduction electrons. When adding a second magnetic moment at a position $\mathbf{r}_{j}$ it couples to the spin density with energy $J\left\langle\mathbf{S}\left(\mathbf{r}_{j}\right) \cdot \mathbf{s}\left(\mathbf{r}_{j}\right)\right\rangle$. The effective RKKY interaction term between the magnetic moments therefore reads

$$
\mathcal{H}_{\mathrm{RKKY}}=-J^{2} \chi\left(\mathbf{r}_{j}-\mathbf{r}_{i}\right) \mathbf{S}\left(\mathbf{r}_{j}\right) \cdot \mathbf{S}\left(\mathbf{r}_{i}\right) .
$$

As a consequence the f-spins of the Kondo lattice model order antiferromagnetically at weak coupling. The energy of this RKKY state is proportional to $E_{\mathrm{RKKY}} \sim J^{2} \rho$, which is exactly the energy scale used in the construction of the Doniach diagram in section 1.3. At half-filling the conduction electrons also form an antiferromagnetic insulator. Doping the system leads to mobile charge carriers and it becomes metallic. In contrast to the heavy Fermi liquid that was discussed before, the f-spins order and do not participate in the transport process. Conduction band electrons make up the Fermi surface, which is why it is called "small" compared to the large Fermi surface encountered before.

\section{Setting the Stage for s-Wave Superconductivity}

Still in the large $J / t$ limit, looking also at second order processes in $t$ leads to a possible effective pairing, which was pointed out by Bodensiek et al. in Ref. [BŽ $\left.{ }^{+} 13\right]$. Considering an electron filling $n<1$, the system consists of singlets and unpaired local spins, which can be interpreted as spinful c-holes. In the configuration shown in figure 2.3 on the left, the singlet can be broken by letting the conduction electron hop to the left, which results in a triplet on a neighboring site. This virtual high energy triplet then decays into a singlet on a neighboring site, which does not have to coincide with the site from the first hopping process as long as the spin of the neighboring hole fits, too (fig. 2.3 right). Therefore, this second order process, which leads to an energy gain of order $t^{2} / J$, binds two holes to a singlet. The pairing happens in the intermediate step at the virtual triplet and is hence local. For this reason, the process gives a quite natural explanation for the existence of s-wave superconductivity which was found in Ref. [BŽ $\left.\mathrm{V}^{+} 13\right]$.

Furthermore, in the supplemental material to their paper [BŽ $\left.\mathrm{V}^{+} 13\right]$, the authors showed that a spin-fluctuation theory, which is based on Eliashberg theory, does not exclude s-wave superconductivity for the Kondo lattice model. This theory includes several uncontrolled approximations, but shows that under very special conditions, which could be met within heavy fermion systems, s-wave superconductivity found in their numerical study can be supported. In the following, some of the key assumptions and approximations of this theory are laid out and checked for applicability to the study by variational cluster approximation that is given in chapter 5 .

The starting point for their theory is the Eliashberg gap equation in non-crossing approximation; a derivation can be found in a book by Plakida [Pla10]. In his book, however, 


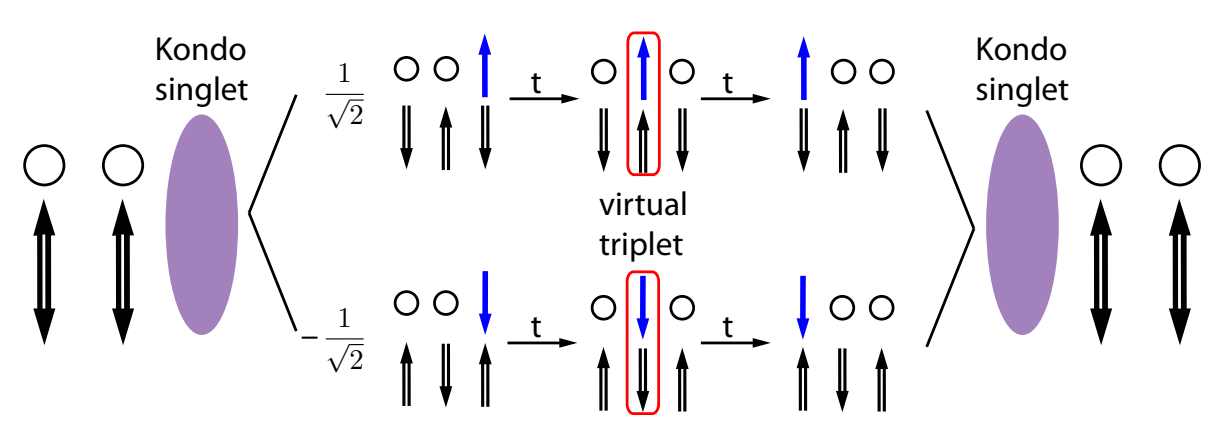

Figure 2.3: Possible second order hopping process leading to an effective local pairing. The Kondo singlet is broken up by the first hopping process into an virtual triplet state. After a second hopping process the Kondo singlet forms at another site. Figure adapted from Ref. $\left[\mathrm{BŽV} \mathrm{V}^{+} 13\right]$.

Plakida uses standard Eliashberg theory for a Hamiltonian with phononic interactions and with spin-fluctuations which couple in a general way to the conduction electrons. For the discussion of the Kondo lattice model one skips the phononic part and specifies the spin-fluctuations to the Kondo term. Even before applying the noncrossing approximation, an important approximation is made in order to obtain the self-energy of Eliashberg theory in the general form using Nambu spinors: In order to arrive at the equation for the self-energy, which serves them as a starting point, one already has to assume that magnetic order is absent. This means that the validity of their theory to the parameter regime of weak and intermediate coupling strengths in the KLM close to half-filling has to be questioned as the system shows antiferromagnetic order. Although the superconducting and antiferromagnetic regions seem to be complementary within DMFT, there is a small region where both phases overlap $\left[\mathrm{BŽV}^{+} 13\right]$. Further approximations are the non-crossing approximation, which consists of assuming that spin fluctuations and electrons propagate independently, and the assumption of a local self-energy. The latter assumption is true in infinite dimensions and depicts an approximation of DMFT, but in VCA the self-energy is cluster-representable and usually non-local. In the end of their derivation, the superconducting gap can be related to the spin susceptibility $\chi_{S}$, which is a quantity that is accessible within DMFT calculations. By furthermore approximating the gap by a step function, it was shown that Cooper pairing can occur if the energy scale of the hybridized bands and the one of the spin-fluctuations are of the same scale $\left[\mathrm{BŽV}^{+} 13\right]$. 



\section{Theoretical Framework 3}

In this chapter, the theoretical concepts and techniques are introduced that are used later to investigate the Hubbard and Kondo lattice model in chapters 4 and 5 .

The first section 3.1 introduces the Green function as it is used in many-body physics. It is shown how to calculate it in Lehmann representation using the efficient Band Lanczos algorithm. There, the notation of "Q matrices" is explained. In subsection 3.1.2 the Gorkov-Green function is introduced which is used in chapter 5 to investigate superconductivity. The concept of tiling a lattice system into a superlattice of clusters is presented in subsection 3.1.3 and a conceptional simple cluster method, so-called cluster perturbation theory, is introduced. In subsection 3.1.4 this technique is extended to spin systems and using supercluster construction it is benchmarked on a paradigmatic quantum spin model.

The second section introduces the self-energy functional theory by Potthoff and a the variational cluster approximation. In subsection 3.2 the self-energy functional theory is revisited and standard variational cluster approximation is shown in subsection 3.2.2. There, some technical details on the evaluation of the self-energy functional, the determination of the stationary point and details on the numerical approach are given. Finally it is shown how VCA can be applied to the Kondo lattice model which also includes the coupling of electrons to immobile spins.

In section 3.3 a survey is given on other numerical techniques that have been used to investigate the Mott transition in two-dimensional Hubbard models and the Kondo lattice model in two dimensions. The results of these techniques are used afterwards as a reference for results obtained in this thesis using variational cluster approaches.

\section{Green Functions and Cluster Perturbation Theory}

In this section the foundations are laid for the derivation of self-energy functional theory and variational cluster approximation in the next section. To calculate the self-energy functional which is essential for VCA, one needs the Green function of the cluster as a main ingredient. Therefore, first the notion of Green functions is introduced in a way they are used in modern many-body physics. There are two different ways of writing the Green function, one via the Lehmann representation and the other one by representing it as a continued fraction expansion. In the first subsection the retarded many-particle Green function is revisited and Lehmann's representation derived; most of this subsection is based on references [Eco90, Mah90, FW03, Nol09].

Instead of using full diagonalization where all eigenenergies and eigenstates of the system in a certain quantum sector are determined, more efficient algorithms based on the Krylov subspace can be used. For the calculation of the Green function the so-called Band Lanczos algorithm will be used and is explained in subsection 3.1.1.

Within VCA, phases with broken symmetries such as superconductivity can be treated. In this case an anomalous Green function, the so-called Gorkov-Green function, is used. 
It is introduced in subsection 3.1.2; this subsection is in addition based on references [Gor58, SDB11].

The concept of a cluster tiling of the lattice system into a superlattice of clusters is explained in subsection 3.1.3. It is used later for VCA, but in this subsection a precursor method, the cluster perturbation theory [GV93, SPPL00] is introduced.

As was shown by Filor and Pruschke [FP14] a generalization of VCA to spin systems is possible but involves severe limitations such as a breakdown of the theory for small temperatures. To fill the technical gap between a spin extension of CPT for a specific well-behaved spin model and this spin VCA, spin CPT is investigated in subsection 3.1.4 and a supercluster construction is tested. As spin CPT turns out to lead to no significant improvement over exact diagonalization, this route is not pursued in the remainder of this thesis. Only a few characteristic results are shown; application of SCPT to more models and additional results are given in the appendix B.

After this excursus to pure spin systems in subsection 3.1.4, the self-energy functional theory and the variational cluster approximation are introduced in the next section for purely electronic models. Afterwards the latter is also applied to a model containing local interactions with spins.

\section{Green Function within Lehmann Representation}

In the following, the retarded two-times Green function is used. For two operators $\hat{A}, \hat{B}$ it is defined as

$$
\begin{aligned}
G_{A B}\left(t, t^{\prime}\right) & =\left\langle\left\langle\hat{A}(t), \hat{B}\left(t^{\prime}\right)\right\rangle\right\rangle \\
& =-i \theta\left(t-t^{\prime}\right)\left\langle\left[\hat{A}(t), \hat{B}\left(t^{\prime}\right)\right]_{-s}\right\rangle,
\end{aligned}
$$

where $\langle\ldots\rangle$ denotes the thermal average and $[\hat{A}, \hat{B}]_{-s}=\hat{A} \hat{B}-s \cdot \hat{B} \hat{A}$ denotes the (anti)commutator for $s=(-) 1$. If both operators are of fermionic type $s=-1$, if one or both operators are bosonic $s=1$. Time dependence of the operators is included in the Heisenberg picture:

$$
\hat{A}(t)=e^{i \hat{K} t} \hat{A} e^{-i \hat{K} t}, \quad \hat{K}=\hat{H}-\mu \hat{N} .
$$

In order to do the thermal average, a trace over the set of eigenstates $|n\rangle$ of $\hat{K}$ has to be done:

$$
\begin{aligned}
G_{A B}\left(t, t^{\prime}\right)= & -i \theta\left(t-t^{\prime}\right) e^{\beta \Omega} \sum_{n}\left\langle n\left|e^{-\beta \hat{K}}\left[\hat{A}(t) \hat{B}\left(t^{\prime}\right)-s \cdot \hat{B}\left(t^{\prime}\right) \hat{A}(t)\right]\right| n\right\rangle, \quad \beta=1 / T \\
= & -i \theta\left(t-t^{\prime}\right) e^{\beta \Omega} \sum_{n, m, m^{\prime}} e^{-\beta E_{n}}\left[e^{i\left(t-t^{\prime}\right)\left(E_{n}-E_{m}\right)}\langle n|\hat{A}| m\rangle\langle m|\hat{B}| n\rangle\right. \\
& \left.-s \cdot e^{-i\left(t-t^{\prime}\right)\left(E_{n}-E_{m^{\prime}}\right)}\left\langle n|\hat{B}| m^{\prime}\right\rangle\left\langle m^{\prime}|\hat{A}| n\right\rangle\right] .
\end{aligned}
$$

In the second line two complete sets $1=\sum_{m}|m\rangle\langle m|$ have been inserted and $\langle n|\hat{A}(t)| m\rangle=$ $e^{i t\left(E_{n}-E_{m}\right)}\langle n|\hat{A}| m\rangle$ was used. A Laplace transformation into frequency space leads to:

$$
\begin{aligned}
G_{A B}(\omega) & =-i \int_{0}^{\infty} d t e^{i(\omega+i \delta) t} e^{\beta \Omega} \sum_{n, m, m^{\prime}} e^{-\beta E_{n}}\left[e^{i t\left(E_{n}-E_{m}\right)} \cdots-s \cdot e^{-i t\left(E_{n}-E_{m^{\prime}}\right)} \ldots\right] \\
& =e^{\beta \Omega} \sum_{n, m, m^{\prime}} e^{-\beta E_{n}}\left[\frac{\langle n|\hat{A}| m\rangle\langle m|\hat{B}| n\rangle}{\omega+i \delta-\left(E_{m}-E_{n}\right)}-s \cdot \frac{\left\langle n|\hat{A}| m^{\prime}\right\rangle\left\langle m^{\prime}|\hat{B}| n\right\rangle}{\omega+i \delta-\left(E_{n}-E_{m^{\prime}}\right)}\right],
\end{aligned}
$$


where $i \delta$ was added to keep the poles off the real axis. At zero temperature only the ground state $|\Omega\rangle$ with energy $E_{0}$ contributes to the set $|n\rangle$ and the Green function reads:

$$
G_{A B}(\omega)=\sum_{m, m^{\prime}}\left[\frac{\langle\Omega|\hat{A}| m\rangle\langle m|\hat{B}| \Omega\rangle}{\omega+i \delta-\left(E_{m}-E_{0}\right)}-s \cdot \frac{\left\langle\Omega|\hat{A}| m^{\prime}\right\rangle\left\langle m^{\prime}|\hat{B}| \Omega\right\rangle}{\omega+i \delta-\left(E_{0}-E_{m^{\prime}}\right)}\right] .
$$

As the techniques that are discussed in the following will be carried out at zero temperature, the discussion in this section focusses now on the zero temperature Green function for electrons. The operators $\hat{A}$ and $\hat{A}$ are therefore chosen to be fermionic creation and annihilation operators $\hat{c}_{\mu}^{(\dagger)}$. In this notation, the composite indices $\mu$ and $\nu$ contain site, spin and orbital information.

There exist a number of sum rules and symmetry relations for Green functions, which will be used in the following. One of the most important ones is the sum rule for the frequency-dependent Green function:

$$
\frac{1}{\pi} \int_{-\infty}^{\infty} G_{A B}(\omega) \mathrm{d} \omega=-i\left\langle[A, B]_{s}\right\rangle
$$

It can be reformulated in terms of $\mathbf{Q}$ matrices to check for convergence of the underlying (Band) Lanczos algorithm (see next paragraphs). Especially for the electron/hole Green function where the operators $A$ and $B$ are fermionic creation and annihilation operators for electrons $A=\hat{c}_{\alpha}^{\dagger}, \hat{c}_{\beta}$ and vice versa, the anticommutator simply amounts to $\delta_{\alpha \beta}$. Considering $2 N$ values of $\alpha$ and $\beta$, e.g., electrons with two spin orientations on $N$ lattice sites in one orbital, the sum rule reads

$$
\operatorname{Tr} \frac{1}{\pi} \int_{-\infty}^{\infty} G^{c}(\omega) \mathrm{d} \omega=2 N
$$

\section{Using Q-Matrices in the Lehmann Representation}

For a finite system of $N$ lattice sites, the Green function is written as

$$
G_{\mu \nu}(\omega)=\left\langle\Omega\left|\hat{c}_{\mu} \frac{1}{\omega-H+E_{0}} \hat{c}_{\nu}^{\dagger}\right| \Omega\right\rangle+\left\langle\Omega\left|\hat{c}_{\nu}^{\dagger} \frac{1}{\omega+H-E_{0}} \hat{c}_{\mu}\right| \Omega\right\rangle
$$

The first term is sometimes called electron part as it contains excitations when adding an electron. Removing an electron can be viewed as adding a hole, which is why the second term is sometimes referred to as the hole part. The Lehman representation of the Green function, which was introduced in the previous subsection reads:

$$
G_{\mu \nu}(\omega)=\sum_{a}\left\langle\Omega\left|\hat{c}_{\mu}\right| a\right\rangle \frac{1}{\omega-E_{a}+E_{0}}\left\langle a\left|\hat{c}_{\nu}^{\dagger}\right| \Omega\right\rangle+\sum_{b}\left\langle\Omega\left|\hat{c}_{\nu}^{\dagger}\right| b\right\rangle \frac{1}{\omega+E_{b}-E_{0}}\left\langle b\left|\hat{c}_{\mu}\right| \Omega\right\rangle .
$$

The split-up into two different sums is reasonable as the introduced states $|a\rangle$ and $|b\rangle$ belong to different quantum sectors of the Hilbert space. In the first sum the states have one additional electron, in the second some one electron less than the ground state. By using the "Q-matrix" notation introduced by Zacher et al. [ZEAH02]:

$$
\begin{array}{ll}
Q_{\mu, a}^{(e)}=\left\langle\Omega\left|\hat{c}_{\mu}\right| a\right\rangle, & Q_{\nu, b}^{(h)}=\left\langle\Omega\left|\hat{c}_{\nu}^{\dagger}\right| b\right\rangle \\
\omega_{a}^{(e)}=E_{a}-E_{0}, & \omega_{b}^{(h)}=E_{0}-E_{b}
\end{array}
$$


one can write the Green function as a product of $\mathbf{Q}$ matrices. In order to simplify the notation, the matrices $\mathbf{Q}^{(h)}$ and $\mathbf{Q}^{(e)}$ are concatenated into one matrix of size $2 N \times$ $\left(L^{(e)}+L^{(h)}\right)$, denoted by $\mathbf{Q}=\mathbf{Q}^{(h)} \oplus \mathbf{Q}^{(e)} . L^{(e / h)}$ is the number of states in $|a / b\rangle$ that enter the respective $\mathbf{Q}^{(e / h)}$ matrices, the factor of two in " $2 N$ " stems from the two spin configurations that have to be included in the operators $c_{\mu}^{(\dagger)}$. After concatenating also the excitation energies of the electron and hole part, the Green function can be written compactly as

$$
G_{\mu \nu}(\omega)=\sum_{m} \frac{Q_{\mu m}^{(e)} Q_{\nu m}^{(e) *}}{\omega-\omega_{n}^{(e)}}+\sum_{n} \frac{Q_{\mu n}^{(h)} Q_{\nu n}^{(h) *}}{\omega-\omega_{n}^{(h)}}=\sum_{n} \frac{Q_{\mu n} Q_{\nu n}^{*}}{\omega-\omega_{n}}
$$

The final step to arrive at a pure matrix notation consists in writing the frequency part into a diagonal matrix

$$
g_{m n}(\omega)=\frac{\delta_{m n}}{\omega-\omega_{n}}=\frac{1}{\omega-\Lambda_{m n}} .
$$

The Green function in Lehmann representation reads now

$$
\mathbf{G}(\omega)=\mathbf{Q g}(\omega) \mathbf{Q}^{\dagger} .
$$

Except for a digression to finite-temperatures in appendix E, the zero-temperature Green function will be used in this thesis. However, it is possible to use the Q-matrix notation also for the finite-temperature Green function [AAPH06b, ZEAH02].

\section{The Lanczos and Band Lanczos Algorithms}

For small clusters, the Hamiltonian matrix can be diagonalized via full diagonalization to obtain all eigenenergies and eigenvalues. However, already for modest system sizes, the matrices which have to be diagonalized become too large to do an exact evaluation. An iterative way to determine the lowest eigenvalue of a matrix is the Lanczos algorithm [Ruh00, Saa03]. In order to apply it, the lowest two eigenvalues of the matrix have to be well separated, otherwise a variant of it, the Band Lanczos algorithm[Fre00, MP89] should be used. The Lanczos algorithm is based on repeatedly applying the Hamiltonian $H$ on an trial state $\left|\phi_{0}\right\rangle$ to project out the lowest eigenstate. In contrast to the power method, where a power of $H$ is applied to an initial state, the Lanczos method works in the so-called Krylov subspace. This space is spanned by the vectors $H^{j}\left|\phi_{0}\right\rangle$ :

$$
\mathfrak{K}=\operatorname{span}\left\{\left|\phi_{0}\right\rangle, H\left|\phi_{0}\right\rangle, H^{2}\left|\phi_{0}\right\rangle, \ldots\right\} .
$$

Starting with a random vector $\left|\phi_{0}\right\rangle$ the next vectors are constructed iteratively via a recursion relation [Dag94] in which they are orthogonalized against the two previous Lanczos vectors:

$$
\left|\phi_{n+1}\right\rangle=H\left|\phi_{n}\right\rangle-\underbrace{\frac{\left\langle\phi_{n}|H| \phi_{n}\right\rangle}{\left\langle\phi_{n} \mid \phi_{n}\right\rangle}}_{a_{n}}\left|\phi_{n}\right\rangle-\underbrace{\frac{\left\langle\phi_{n} \mid \phi_{n}\right\rangle}{\left\langle\phi_{n-1} \mid \phi_{n-1}\right\rangle}}_{b_{n}^{2}}\left|\phi_{n-1}\right\rangle, \quad\left|\phi_{-1}\right\rangle=0, b_{0}=0 .
$$

Therefore, the vectors $\phi_{n-1}, \phi_{n}, \phi_{n+1}$ are mutually orthogonal by construction. However, orthogonality can be lost after a large number of iterations as numerical errors sum up. There are ways to cure the loss of orthogonality [Ruh00, Saa03], but in most cases the lowest eigenvalue has converged before these losses of orthogonality show up. 
When using normalized Lanczos states, the Hamiltonian expressed in the Lanczos basis has the tridiagonal form

$$
\mathfrak{H}=\left(\begin{array}{cccccc}
a_{0} & b_{1} & 0 & 0 & \ldots & 0 \\
b_{1} & a_{1} & b_{2} & 0 & \ldots & 0 \\
0 & b_{2} & a_{2} & b_{3} & \ldots & 0 \\
\vdots & \vdots & \vdots & \vdots & \ddots & \vdots \\
0 & 0 & 0 & 0 & \ldots & a_{N}
\end{array}\right)
$$

which allows the use of fast routines to diagonalize this matrix.

A stop criterion for this iterative procedure is the convergence of the lowest eigenvalue of $\mathfrak{H}$. The lowest eigenvalue $\tilde{E}_{0}$ of the matrix in the Lanczos basis and its corresponding eigenvector $\tilde{\Omega}$ are then used as an approximation of $E_{0}$ and $|\Omega\rangle$. In practice, during the Lanczos recursion only the coefficients $\left\{a_{n}\right\}$ and $\left\{b_{n}\right\}$ as well as three basis states $\phi_{n-1}, \phi_{n}, \phi_{n+1}$ have to be stored to calculate $\tilde{E}_{0}$. To calculate $|\tilde{\Omega}\rangle$, one has to do a second run with the same initial vector $\left|\phi_{0}\right\rangle$.

In order to use the semi-analytical method of calculating the self-energy functional in VCA (see section 3.2.2), the Green function has to be written in the Lehmann representation. When resorting to the Q-matrix notation the so-called Band Lanczos algorithm [Fre00, MP89] can be used, where more than one initial vector is used to span the Krylov subspace:

$$
\mathcal{H}=\operatorname{span}\left\{\left|\phi_{1}\right\rangle,\left|\phi_{2}\right\rangle, \ldots,\left|\phi_{p}\right\rangle, H\left|\phi_{1}\right\rangle, H\left|\phi_{2}\right\rangle, \ldots, H\left|\phi_{p}\right\rangle, \ldots,(H)^{K}\left|\phi_{1}\right\rangle, \ldots,(H)^{K}\left|\phi_{p}\right\rangle\right\} .
$$

Similar to the simple Lanczos algorithm, the algorithm starts with normalized states

$$
\left|\phi_{\mu}\right\rangle=\frac{c_{\mu}^{\dagger}|\Omega\rangle}{\sqrt{\left\langle\Omega\left|c_{\mu} c_{\mu}^{\dagger}\right| \Omega\right\rangle}} .
$$

A recursion relation is used to then produce the next candidate vectors, which are orthogonalized against previous Lanczos vectors. This includes two orthogonality conditions: At each iteration $j$, the Lanczos vectors $\Phi_{j}=\left[\left|\phi_{1}\right\rangle\left|\phi_{2}\right\rangle \ldots\left|\phi_{j}\right\rangle\right]$ have to be orthogonal:

$$
\Phi_{j}^{*} \Phi_{j^{\prime}}=\mathbb{1}_{j} .
$$

Additionally, the candidate vectors $\left|\tilde{\phi}_{j+1}\right\rangle, \ldots,\left|\tilde{\phi}_{j+p_{j}}\right\rangle$ for the next iteration are constructed to be orthogonal to the already existing Lanczos vectors:

$$
\Phi_{j}^{*}\left|\tilde{\phi}_{k}\right\rangle=0, \quad \forall k=j+1, \ldots, j+p_{j} .
$$

Many vectors are converging in parallel during the recursion scheme. It is therefore possible that after an iteration $j$ the new created vector $\left|\tilde{\phi}_{k}\right\rangle$ is linear dependent to previous Lanczos vectors. For the simple Lanczos algorithm the recursion would be stopped at this point, as the Krylov space is already spanned by the previous vectors. In case of the Band Lanczos method, this indicates that one of the vectors is converged, but the rest of the $p$ vectors can still be improved in the next iterations. Therefore, the current Lanczos vector $\left|\tilde{\phi}_{k}\right\rangle$ has to be deflated and the algorithm continues with $p-1$ vectors:

$$
\begin{array}{r}
\left|\phi_{1}\right\rangle, \ldots,\left|\phi_{p}\right\rangle, \ldots,(H)^{j}\left|\phi_{1}\right\rangle, \ldots,(H)^{j}\left|\phi_{k-1}\right\rangle,(H)^{j}\left|\phi_{k+1}\right\rangle, \ldots,(H)^{j}\left|\phi_{p}\right\rangle \\
(H)^{j+1}\left|\phi_{1}\right\rangle, \ldots,(H)^{j+1}\left|\phi_{k-1}\right\rangle,(H)^{j+1}\left|\phi_{k+1}\right\rangle, \ldots,(H)^{j+1}\left|\phi_{p}\right\rangle, \ldots
\end{array}
$$


This means, that any time a deflation has to be performed, the number of newly created candidates $p_{j}$ at iteration $j$ is reduced by one. In order to decide when to perform a deflation, several criteria can be used. Here, the norm of the new Lanczos vector is used as a deflation criterion. Instead of deleting the vector for any non-zero overlap (so-called exact deflation), a threshold value $d_{\text {tol }}$ is used as a tolerance. Without deflation the projected Hamiltonian has a diagonal band. Each time a linear dependent candidate vector is deleted, the bandwidth of the projected band matrix is reduced. As a consequence of this non-exact deflation, at each deflation additional off-band elements occur in the projected matrix.

The simple Lanczos algorithm is recovered from the Band Lanczos algorithm in the case of only one start vector. In contrast to the simple Lanczos algorithm, the deflation rule prevents artificial degeneracy due to numerical round-off errors (so-called ghost states). In the Band Lanczos algorithm two Lanczos vectors which converge to the same eigenvalue, correspond to a true degeneracy of this eigenvalue. It can be hence used to check for energetic degeneracy of the ground state.

When comparing the calculation of the Green function with the Band Lanczos algorithm to the calculation with the ordinary Lanczos method (see appendix A), the Band Lanczos algorithm is faster. As the states $\left|\phi_{\mu}\right\rangle$ converge in parallel, only two runs (one for the electron- and one for the hole-part of the Green function) of the Band Lanczos algorithm with $2 N$ start vectors are needed. With the simple Lanczos algorithm each matrix element of the Green matrix has to be calculated separately, which leads to $N *(N+1) / 2$ runs. Thereby, using the Band Lanczos algorithm is roughly a factor $N^{2}$ faster. The drawback of the method lies in its larger memory requirements. Instead of three vectors in case of the simple Lanczos algorithm, $\left(2 N_{c}+1\right)$ vectors have to be stored during the iterations of the Band Lanczos algorithm [Fre00, Sén08a].

In practice, some parameters such as the deflation tolerance or the convergence criterion have to be adjusted properly to avoid orthogonality leaks and to ensure the convergence of the algorithm. For most cases, a deflation tolerance of $d_{\text {tol }}=10^{-9}$ and a check of the convergence up to $5 * 10^{-11}$ of the lowest eigenvalue were sufficient. The convergence criterion is checked after the first $\tilde{p}=4 \cdot p$ iterations at each iteration. In addition, a separate check for the resulting total weight of the Green matrix has been included. Depending on the outcome of this check, the values of $\tilde{p}$ and $d_{\text {tol }}$ are then changed to improve the result.

\section{Calculating the Green function in Q-matrix notation}

Compared to the simple Lanczos algorithm, one of the advantages of the Band Lanczos algorithm is the possibility to calculate the Green function within the above mentioned Q-matrix formalism. In this formalism, the excited states $|n\rangle$ and $|m\rangle$ are the same within the Q matrix. Within the ordinary Lanczos algorithm every element of the Green matrix is calculated with a different approximate set of vectors which does not lead to a common set of poles for all matrix elements. Including all different sets of poles for the $2 N$ Lanczos runs would result in a huge Q-matrix. Its diagonalization would be barely possible for larger clusters. Within the Band Lanczos algorithm all vectors $\phi_{\mu}$ converge in parallel. Hence a common set of poles is built up.

At the end of the Band Lanczos run, the cluster Hamiltonian $H^{\prime}$ is projected on the Krylov subspace spanned by $R$ Lanczos vectors $|k\rangle$, leading to the matrix T. After diagonalizing 
this band-like matrix, eigenvalues $\theta_{i}$ and (normalized) eigenvectors $\mathbf{z}_{i}$ are obtained, which are then used to construct the Lanczos eigenstates

$$
|\tilde{m}\rangle=\sum_{k} z_{\tilde{m} k}|k\rangle \text {. }
$$

Projecting the vectors $\left|\phi_{\mu}\right\rangle$ on the eigenstates of $\mathbf{T}$ leads to the $Q^{(e / h)}$ matrices. They can be computed as

$$
\begin{aligned}
Q_{\mu \tilde{m}} & =\left\langle\phi_{\mu} \mid \tilde{m}\right\rangle \\
& =\sum_{k} z_{\tilde{m} k}\left\langle\bar{k}_{\mu} \mid k\right\rangle=\sum_{k} z_{\tilde{m} k} T_{\mu k}
\end{aligned}
$$

The corresponding eigenenergies amount to $\omega_{m}= \pm\left(\theta_{m}-E_{0}\right)$. As a non-zero deflation tolerance is used, the sum cannot be restricted and has to be performed over all $k$.

For the calculation of $G_{\mu \nu, e / h}(\omega)$ one thereby uses an approximative set of eigenvectors $|\tilde{m}\rangle$ and hence introduces an error. States which converge fast are responsible for excitations with large weight. As these are dominant in the calculation of the self-energy functional $\Omega$ [AAPH06b, Pot03a, Pot03b], the error introduced by using the Band Lanczos technique are small. The concatenated Q-matrix has now a smaller dimension $\left(2 N_{c} \times R\right)$ with $R<$ $\left(L^{(e)}+L^{(h)}\right)$. Nevertheless, as long as orthogonality of the Lanczos vectors is preserved, the relation $\mathbf{Q Q}^{\dagger}=\mathbb{1}_{2 N_{c} \times 2 N_{c}}$ is still valid as it amounts to the commutation relations $\left\{c_{\mu}, c_{\nu}^{\dagger}\right\}=\delta_{\mu \nu}[\mathrm{Sén08 \textrm {a } ]}$.

\section{The Gorkov-Green Function and Nambu Formalism}

In order to describe superconductivity one has to allow for the creation and annihilation of pairs of electrons (Cooper pairs). The concept of Green functions has therefore been extended by L.P. Gorkov [Gor58] and Y. Nambu [Nam60] to so-called anomalous or Nambu-Gorkov-Green functions:

$$
\begin{aligned}
& \mathcal{F}_{a b}(\omega)=\sum_{m}\left\{\frac{\left\langle\Omega\left|c_{a \uparrow}\right| m\right\rangle\left\langle m\left|c_{b \downarrow}\right| \Omega\right\rangle}{\omega-\left(E_{m}-E_{0}\right)+i \eta}+\frac{\left\langle\Omega\left|c_{b \downarrow}\right| m\right\rangle\left\langle m\left|c_{a \uparrow}\right| \Omega\right\rangle}{\omega+\left(E_{m}-E_{0}\right)-i \eta}\right\} \\
& \mathcal{F}_{a b}^{\dagger}(\omega)=\sum_{m}\left\{\frac{\left\langle\Omega\left|c_{a \downarrow}^{\dagger}\right| m\right\rangle\left\langle m\left|c_{b \uparrow}^{\dagger}\right| \Omega\right\rangle}{\omega-\left(E_{m}-E_{0}\right)+i \eta}+\frac{\left\langle\Omega\left|c_{b \uparrow}^{\dagger}\right| m\right\rangle\left\langle m\left|c_{a \downarrow}^{\dagger}\right| \Omega\right\rangle}{\omega+\left(E_{m}-E_{0}\right)-i \eta}\right\}
\end{aligned}
$$

Here, it is important to note, that the states $|m\rangle$ are those with one additional electron and one electron less than the ground state's. Splitting the Green function up into two with one spin species each:

$$
\begin{aligned}
& \mathcal{G}_{a b}^{\uparrow}(\omega)=\sum_{m}\left\{\frac{\left\langle\Omega\left|c_{a \uparrow}\right| m\right\rangle\left\langle m\left|c_{b \uparrow}^{\dagger}\right| \Omega\right\rangle}{\omega-\left(E_{m}-E_{0}\right)+i \eta}+\frac{\left\langle\Omega\left|c_{b \uparrow}^{\dagger}\right| m\right\rangle\left\langle m\left|c_{a \uparrow}\right| \Omega\right\rangle}{\omega+\left(E_{m}-E_{0}\right)-i \eta}\right\} \\
& \mathcal{G}_{a b}^{\downarrow}(\omega)=\sum_{m}\left\{\frac{\left\langle\Omega\left|c_{a \downarrow}^{\dagger}\right| m\right\rangle\left\langle m\left|c_{b \downarrow}\right| \Omega\right\rangle}{\omega-\left(E_{m}-E_{0}\right)+i \eta}+\frac{\left\langle\Omega\left|c_{b \downarrow}^{\dagger}\right| m\right\rangle\left\langle m\left|c_{a \downarrow}\right| \Omega\right\rangle}{\omega+\left(E_{m}-E_{0}\right)-i \eta}\right\}
\end{aligned}
$$

leads the to the Nambu-Gorkov matrix

$$
\mathrm{G}^{\text {Gorkov }}=\left[\begin{array}{cc}
\mathcal{G}^{\uparrow} & \mathcal{F} \\
\mathcal{F}^{\dagger} & \mathcal{G}^{\downarrow}
\end{array}\right] .
$$


This can be rewritten with the operators $\mathbf{A}_{a}^{(e)}=\left(\begin{array}{c}c_{a \uparrow} \\ c_{a \downarrow}^{\dagger}\end{array}\right), \quad \mathbf{A}_{a}^{(h)}=\left(\begin{array}{c}c_{a \uparrow}^{\dagger} \\ c_{a \downarrow}\end{array}\right)$ as:

$$
\mathbf{G}^{\text {Gorkov }}=\left\langle\left\langle\hat{\mathbf{A}}_{a}^{(e)}(t) \hat{\mathbf{A}}_{b}^{(e) \dagger}\left(t^{\prime}\right)\right\rangle\right\rangle+\left\langle\left\langle\hat{\mathbf{A}}_{b}^{(h) *}\left(t^{\prime}\right) \hat{\mathbf{A}}_{a}^{(h) T}(t)\right\rangle\right\rangle
$$

Adopting the „Q-matrix notation“ from 3.1.1 for the Nambu-Gorkov matrix leads to

$$
\mathbf{Q}_{a n}^{(e, N G)}=\left(\begin{array}{c}
\left\langle\Omega\left|c_{a \uparrow}\right| n\right\rangle \\
\left\langle\Omega\left|c_{a \downarrow}^{\dagger}\right| n\right\rangle
\end{array}\right), \quad \mathbf{Q}_{a m}^{(h, N G)}=\left(\begin{array}{c}
\left\langle\Omega\left|c_{a \uparrow}^{\dagger}\right| m\right\rangle \\
\left\langle\Omega\left|c_{a \downarrow}\right| m\right\rangle
\end{array}\right)
$$

Indeed, carrying out this calculation gives

$$
\begin{aligned}
\mathbf{Q}_{a}^{(e, N G)} \hat{\mathbf{Q}}_{b}^{(e, N G) \dagger}= & \left(\begin{array}{cc}
\left\langle\Omega\left|c_{a \uparrow}\right| n\right\rangle\left(\left\langle\Omega\left|c_{b \uparrow}\right| n\right\rangle\right)^{*} & \left\langle\Omega\left|c_{a \uparrow}\right| n\right\rangle\left(\left\langle\Omega\left|c_{b \downarrow}^{\dagger}\right| n\right\rangle\right)^{*} \\
\left\langle\Omega\left|c_{a \downarrow}^{\dagger}\right| n\right\rangle\left(\left\langle\Omega\left|c_{b \uparrow}\right| n\right\rangle\right)^{*} & \left\langle\Omega\left|c_{a \downarrow}^{\dagger}\right| n\right\rangle\left(\left\langle\Omega\left|c_{b \downarrow}^{\dagger}\right| n\right\rangle\right)^{*}
\end{array}\right) \\
\mathbf{Q}_{a}^{(h, N G) *} \hat{\mathbf{Q}}_{b}^{(h, N G) T}= & \left(\begin{array}{ll}
\left\langle\Omega\left|c_{b \uparrow}^{\dagger}\right| m\right\rangle\left(\left\langle\Omega\left|c_{a \uparrow}^{\dagger}\right| m\right\rangle\right)^{*} & \left\langle\Omega\left|c_{b \downarrow}\right| m\right\rangle\left(\left\langle\Omega\left|c_{a \uparrow}^{\dagger}\right| m\right\rangle\right)^{*} \\
\left\langle\Omega\left|c_{b \uparrow}^{\dagger}\right| m\right\rangle\left(\left\langle\Omega\left|c_{a \downarrow}\right| m\right\rangle\right)^{*} & \left\langle\Omega\left|c_{b \downarrow}\right| m\right\rangle\left(\left\langle\Omega\left|c_{a \downarrow}\right| m\right\rangle\right)^{*}
\end{array}\right)
\end{aligned}
$$

One should note that $|n\rangle$ are states in the quantum sectors $\left(N_{\text {tot }}^{e}+1, S_{\text {tot }}^{z}+1 / 2\right)$ and $\left(N_{\text {tot }}^{e}-1, S_{\text {tot }}^{z}+1 / 2\right)$, whereas $|m\rangle$ are out of $\left(N_{\text {tot }}^{e}+1, S_{\text {tot }}^{z}-1 / 2\right)$ and $\left(N_{\text {tot }}^{e}-1, S_{\text {tot }}^{z}-1 / 2\right)$. One therefore still can split the calculation up into two Band Lanczos runs.

Therefore:

$$
\mathbf{Q}_{\mathrm{Nambu}}=\left(\begin{array}{ll}
\mathbf{Q}^{(e, N G)} & \mathbf{Q}^{(h, N G) *}
\end{array}\right), \quad \boldsymbol{\Lambda}_{\mathrm{Nambu}}=\left(\begin{array}{cl}
\omega_{n} & -\omega_{m}
\end{array}\right), \quad \mathbf{g}_{\mathrm{Nambu}}(\omega)=\frac{1}{\omega-\boldsymbol{\Lambda}_{\mathrm{Nambu}}}
$$

And finally:

$$
\mathbf{G}_{\text {Gorkov }}=\mathbf{Q}_{\mathrm{N}} \mathbf{g}_{\mathrm{N}}(\omega) \mathbf{Q}_{\mathrm{N}}^{\dagger} \text {. }
$$

One has to be careful with the down-spin part of the Green function as the block in Nambu-Gorkov notation amounts to $\left\langle\left\langle c_{a \downarrow}^{\dagger} c_{a \downarrow}\right\rangle\right\rangle$. Anticommuting these operators leads to a change in sign and to additional terms for local operators in the Hamiltonian, which can be accounted for in the $\mathbf{V}$ matrix. For example anticommutation of the operators in $\mathcal{G}^{\downarrow}$ leads in case of the chemical potential $\mu$ to a constant term $L \cdot\left(\mu-\mu^{\prime}\right)$.

\section{Particle-Hole Transformation to avoid Nambu Formulation}

As long, as one is not interested in any non-local spin-flip terms or spin-flipping Weiss fields, it is possible to avoid rewriting everything in Nambu's formulation by using an unitary transformation instead. Changing particle creation operators for one spin species, say down spin electrons, into hole destruction operators

$$
\hat{c}_{i \uparrow} \rightarrow \hat{c}_{i}, \quad \hat{c}_{i \downarrow}^{\dagger} \rightarrow \hat{d}_{i},
$$

changes a standard Hubbard model as follows

$$
\mathcal{H}_{\text {Hubbard }}=\sum_{\langle i, j\rangle} t_{i j}\left(c_{i}^{\dagger} c_{j}-d_{i}^{\dagger} d_{j}\right)-U \sum_{i} n_{i}^{c} n_{i}^{d}-\mu \sum_{i}\left(n_{i}^{c}-n_{i}^{d}\right)+\sum_{i}\left(U n_{i}^{c}-\mu\right) .
$$

The interaction is now attractive and the one-particle terms changed; the constant terms have to be taken care of when calculating expectation values or the self-energy functional. So far the Hamiltonian has only been rewritten. The advantage of the transformation lies 
in writing additional superconducting Weiss fields now as hybridization terms between $c$ and $d$ operators:

$$
\mathcal{H}_{\text {Weiss }, \mathrm{SC}}=D \sum_{i, j} \Delta_{i j}\left(c_{i}^{\dagger} d_{j}+d_{i}^{\dagger} c_{j}\right), \quad \mathcal{H}_{\text {Weiss }, \mathrm{AF}}=M \sum_{i, j} e^{i \mathbf{Q} \cdot \mathbf{R}}\left(n_{i}^{c}+n_{i}^{d}\right) .
$$

Therefore the matrix of one-body terms changes:

$$
\left(\begin{array}{cc}
\mathbf{t}_{\uparrow}-\mu+e^{i \mathbf{Q} \cdot \mathbf{R}} M & 0 \\
0 & \mathbf{t}_{\downarrow}-\mu-e^{i \mathbf{Q} \cdot \mathbf{R}} M
\end{array}\right) \longrightarrow\left(\begin{array}{cc}
\mathbf{t}_{\uparrow}+U-\mu+e^{i \mathbf{Q} \cdot \mathbf{R}} M & \boldsymbol{\Delta} \\
\boldsymbol{\Delta} & \mu-\mathbf{t}_{\downarrow}+e^{i \mathbf{Q} \cdot \mathbf{R}} M
\end{array}\right)
$$

In both cases, using the Gorkov-Green function and Nambu formulation or doing a particle-hole transformation, the variational cluster approximation can be used to investigate superconductivity. Before turning to the self-energy functional theory in section 3.2 and finally to the variational cluster approximation in 3.2.2, in the following subsection the cluster perturbation theory is introduced.

\section{Cluster Perturbation Theory: Introducing the Cluster Green}

\section{Function

Cluster methods such as the variational cluster approximation discussed in section 3.2.2 use a tiling of the original lattice system into a superlattice of clusters. In this subsection, which is based on reference [Sén08a], the concept of a cluster tiling and its consequences for the (cluster) Green function are discussed. In principle, these clusters can differ, but for the moment a tiling with identical clusters is considered, see figure 3.1.
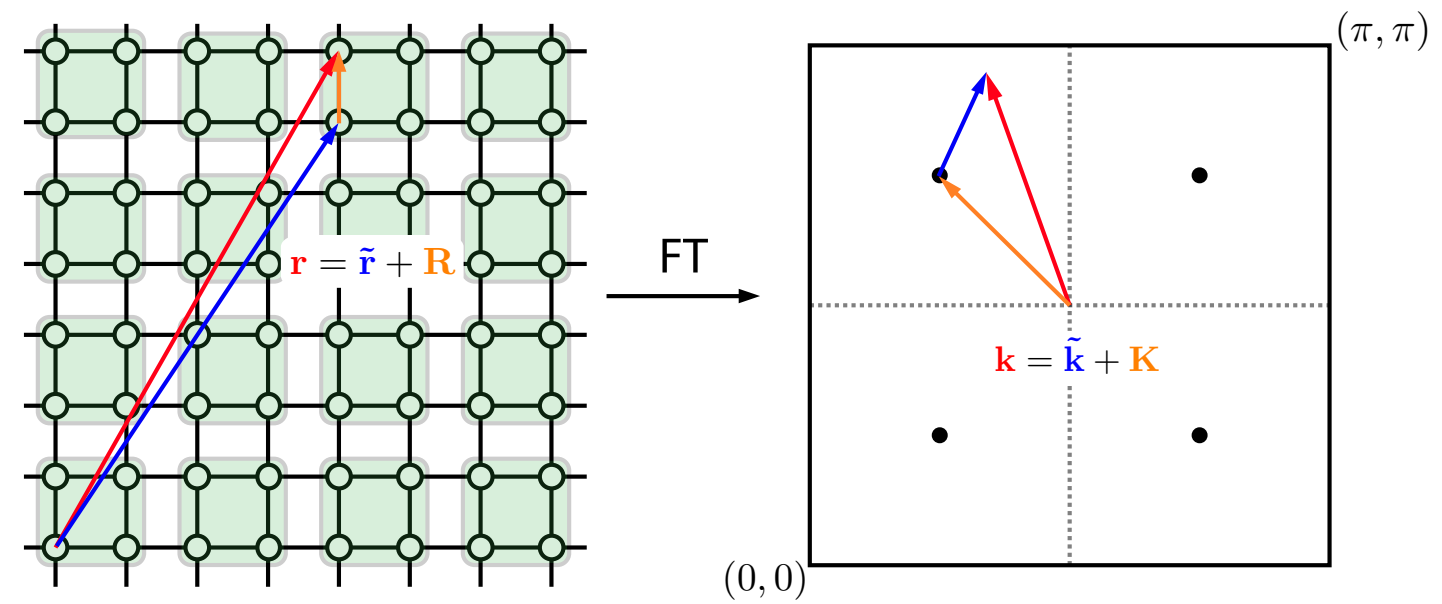

Figure 3.1: A two-dimensional square lattice is tiled into a superlattice of identical $2 \times 2$ clusters. Each lattice vector $\mathbf{r}$ can be then decomposed into a superlattice vector $\tilde{\mathbf{r}}$ and a cluster vector $\mathbf{R}$. After a Fourier transformation to momentum space each vector $\mathbf{k}$ can be similarly decomposed into vectors $\mathbf{K}$ and $\tilde{\mathbf{k}}$.

Therefore, new coordinates are introduced, which are made up of cluster vectors $\tilde{\mathbf{R}}$ and superlattice coordinates $\tilde{\mathbf{r}}$. With this tiling of the lattice the overall translational symmetry is broken, but there is still translational symmetry within the new superlattice. This 
symmetry is exploited by doing a partial Fourier transformation with respect to the superlattice coordinate $\tilde{\mathbf{r}} \longrightarrow \tilde{\mathbf{k}}$. If one would do a full Fourier transformation, the Brillouin zone would be made up of reciprocal superlattice vectors $\tilde{\mathbf{k}}$ and of vectors $\mathbf{K}$ which belong both to the original and the superlattice Brillouin zone, see figure 3.1. When applying the partial Fourier transformation both to the clustered and the original lattice system, all one-body terms that differ between these two systems can be cast into a $\tilde{\mathbf{k}}$-dependent matrix $V(\tilde{\mathbf{k}})$.

Within the so-called cluster perturbation theory (CPT) [GV93, SPPL00], a precursor of the variational cluster approximation which will be discussed later, this matrix enters the calculation of the Green function in first order perturbation theory as:

$$
\mathbf{G}_{C P T}^{-1}(\omega, \mathbf{k})=\mathbf{G}^{\prime-1}(\omega)-\mathbf{V}(\mathbf{k}) .
$$

Here, $\mathbf{G}^{\prime}$ denotes the cluster Green function, which can be expressed by the Q-matrices introduced before. The CPT Green function can then be written as [AAPH06a]:

$$
\begin{aligned}
\mathbf{G}_{C P T}(\omega, \mathbf{k}) & =\frac{1}{\left(\mathbf{Q g}(\omega) \mathbf{Q}^{\dagger}\right)^{-1}-\mathbf{V}(\tilde{\mathbf{k}})}, \quad \mathbf{g}(\omega)=(\omega-\mathbf{\Lambda})^{-1} \\
& =\mathbf{Q g}(\omega) \mathbf{Q}^{\dagger}+\mathbf{Q g}(\omega) \mathbf{Q}^{\dagger} \mathbf{V}(\mathbf{k}) \mathbf{Q g}(\omega) \mathbf{Q}^{\dagger}+\ldots \\
& =\mathbf{Q}\left(\mathbf{g}(\omega)+\mathbf{g}(\omega) \mathbf{Q}^{\dagger} \mathbf{V}(\mathbf{k}) \mathbf{Q g}(\omega)+\ldots\right) \mathbf{Q}^{\dagger} \\
& =\mathbf{Q} \frac{1}{\mathbf{g}^{-1}(\omega)+\mathbf{Q}^{\dagger} \mathbf{V}(\mathbf{k}) \mathbf{Q}} \mathbf{Q}^{\dagger}
\end{aligned}
$$

The eigenvalues of the matrix $\mathbf{L}=\mathbf{\Lambda}+\mathbf{Q}^{\dagger} \mathbf{V}(\tilde{\mathbf{k}}) \mathbf{Q}$ in the denominator correspond to the poles of the CPT Green function. It can be diagonalized using a hermitian matrix $\mathbf{U}$ :

$$
\mathbf{M}(\tilde{\mathbf{k}})=\mathbf{U}(\tilde{\mathbf{k}}) \mathbf{L}(\tilde{\mathbf{k}}) \mathbf{U}(\tilde{\mathbf{k}})^{\dagger} \quad, \mathbf{M}_{m n}=\delta_{m n} \bar{\omega}_{m} .
$$

Thereby the CPT Green function can be written in a form similar to $\mathbf{Q}$ matrix notation of the cluster Green function:

$$
\begin{aligned}
\mathbf{G}_{\mathrm{CPT}}(\tilde{\mathbf{k}}, \omega) & =(\mathbf{Q U}(\tilde{\mathbf{k}})) \frac{1}{\omega-\mathbf{M}(\tilde{\mathbf{k}})}(\mathbf{Q U}(\tilde{\mathbf{k}}))^{\dagger} \\
& =\overline{\mathbf{Q}}(\tilde{\mathbf{k}}) \frac{1}{\omega-\mathbf{M}(\tilde{\mathbf{k}})} \overline{\mathbf{Q}}(\tilde{\mathbf{k}})^{\dagger}
\end{aligned}
$$

In order to obtain the spectral function of the lattice system, one first of all has to restore translational invariance that is broken by the cluster tiling. Following the reperiodization prescription of Sénéchal et al. [SPPL00], this can be done approximatively via

$$
\begin{aligned}
G_{\text {rep. }}(\mathbf{k}, \omega) & =\frac{1}{L_{c}} \sum_{\mathbf{R}, \mathbf{R}^{\prime}} \mathrm{e}^{i \mathbf{k} \cdot\left(\mathbf{R}-\mathbf{R}^{\prime}\right)}\left(\mathbf{G}_{\mathrm{CPT}}\right)_{\mathbf{R}, \mathbf{R}^{\prime}}(\mathbf{k}, \omega) \\
& =\hat{Q}(\mathbf{k}) \frac{1}{\omega-\mathbf{M}(\mathbf{k})} \hat{Q}^{\dagger}(\mathbf{k})
\end{aligned}
$$

The matrices $\hat{\mathbf{Q}}(\mathbf{k})$ are the "periodized" $\overline{\mathbf{Q}}$ matrices:

$$
\hat{Q}_{l}(\mathbf{k})=\mathbf{P}_{m}^{T}(\mathbf{k}) \bar{Q}_{m l}(\mathbf{k}), \quad \mathbf{P}_{m}(\mathbf{k})=\frac{1}{\sqrt{L_{c}}} \mathrm{e}^{i \mathbf{k} \cdot \mathbf{R}_{m}}
$$


where $L_{c}$ denotes the number of lattice sites inside the cluster.

Together with the partial Fourier transformation with respect to the superlattice, the full Fourier transformation should be obtained in order to fully restore translational symmetry, that is

$$
G_{\mathbf{k}, \mathbf{k}^{\prime}}(\omega)=\frac{1}{N_{c} L_{c}} \sum_{\mathbf{R}^{\prime}, \tilde{\mathbf{R}}^{\prime}} \mathrm{e}^{i \mathbf{k} \cdot\left(\mathbf{R}^{\prime}-\tilde{\mathbf{R}}^{\prime}\right)} G_{\mathbf{R}^{\prime}, \tilde{\mathbf{R}}^{\prime}}\left(\mathbf{k}^{\prime}, \omega\right) .
$$

Here $N_{c}$ denotes the number of clusters of the lattice tiling. The reperiodization prescription amounts to taking only the diagonal part $G_{\text {rep. }}(\mathbf{k}, \omega)=\delta_{\mathbf{k}, \mathbf{k}^{\prime}} G_{\mathbf{k k}^{\prime}}(\omega)$ and setting the off-diagonal parts to zero. With this notation the spectral function reads

$$
\begin{aligned}
A(\mathbf{k}, \omega) & =-2 \lim _{\eta \rightarrow 0} \operatorname{Im} G_{\text {rep. }}(\mathbf{k}, \omega+i \eta) \\
& =-2 \lim _{\eta \rightarrow 0} \operatorname{Im}\left(\sum_{l} \frac{\left|\hat{Q}_{l}(\mathbf{k})\right|^{2}}{\omega+i \eta-\hat{\omega}_{l}}\right) \\
& =2 \lim _{\eta \rightarrow 0} \sum_{l} \frac{\left|\hat{Q}_{l}(\mathbf{k})\right|^{2} \eta}{\left(\omega-\hat{\omega}_{l}\right)^{2}+\eta^{2}} .
\end{aligned}
$$

Thereby, the spectral function is given by a sum of Lorentz peaks at positions $\hat{\omega}_{l}$ with weights $\left|\hat{Q}_{l}(\mathbf{k})\right|^{2}$. When plotting the spectral function, a finite broadening $\eta \sim 0.02-0.1$ is added to visualize the peaks.

In the next section, variational cluster approximation will be discussed. There, after a variational principle is applied, an approximated lattice Green function is found, from which the spectral function can be obtained according to the formulae that were shown in this subsection. Although it is in principle possible to reperiodize the self-energy instead of the Green function (both quantities are connected via the Dyson equation), it was shown in reference [Sén08a], that a reperiodization of the self-energy leads to inferior results, such as spectral weight in the Mott gap in case of the 1D Hubbard model. Before turning to self-energy functional theory and the variational cluster approximation, it is shown in the next subsection how cluster perturbation theory can be adapted to treat pure spin systems. There, the concept of supercluster constructions is used, which is explained in the following for standard CPT on the 1D Hubbard model.

\section{Supercluster Construction within Cluster Perturbation Theory}

In the following the 1D Hubbard model will be investigated with cluster perturbation theory (CPT) using superclusters. This was done first by Sénéchal et al. in 2002 [SPP02] and most of the notations are adopted here. Although CPT has been broadly applied to a lot of different model systems of strongly correlated electrons, the supercluster construction has not been used much since. Before the idea of using CPT with superclusters is transferred to spin systems in the next subsection, the technique and some numerical details are revisited here for the 1D Hubbard model.

Due to Umklapp scattering the 1D Hubbard model is gapped for all finite interaction strengths $U / t>0\left[\mathrm{EFK}^{+} 05\right]$. It can be solved exactly using the so-called Bethe ansatz [LW68, EFK ${ }^{+}$05], which goes back to Bethe who applied this way of parametrizing the eigenvectors to solve the one-dimensional Heisenberg model [Bet31].

One of the most important entities of this technique is the Green function $G(\mathbf{k}, \omega)$ and the spectral function $A(\mathbf{k}, \omega)=-2 \lim _{\eta \rightarrow 0} \operatorname{Im} G(\mathbf{k}, \omega+i \eta)$. Usually, one either uses a 
grand canonical formulation, where the chemical potential $\mu$ is already included in the Hamiltonian, or one shifts the spectral function by $\mu$. Here, the unshifted cluster Green functions and their spectral functions are used to construct a supercluster and to determine an effective chemical potential for the supercluster afterwards. However, it should be noted that compared to the variational cluster approximation, no variational scheme is applied within CPT. The parameters of the original system and the clustered reference system only differ in the inter-cluster hopping terms, which are treated in the first order perturbation theory, see figure 3.2.

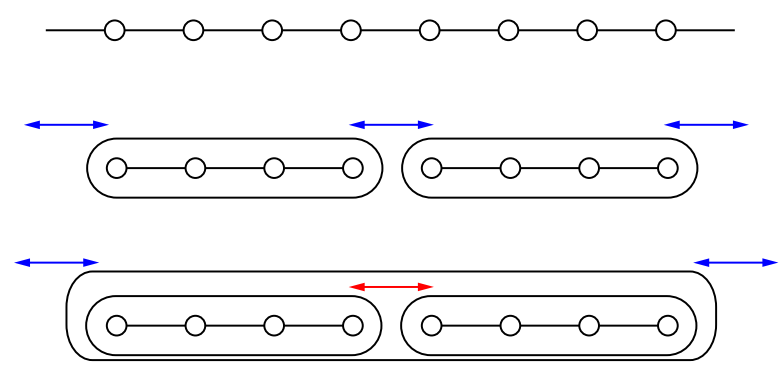

Figure 3.2: Tiling of the infinite 1D model (top) into identical clusters, which are then combined into superclusters (bottom) or used as single clusters (middle) to approximate the Green function via cluster perturbation theory. The arrows indicate inter-cluster hopping terms, which are treated in first order perturbation theory. Blue arrows which connect (super-)clusters lead after partial Fourier transformation to a k-dependence of $G(\mathbf{k}, \omega)$.

To derive the density of states (DOS) from the Green function, one usually integrates over k-space. After integrating the density of states from $-\infty$ to the chemical potential $\mu$, the electron density $n$ is obtained:

$$
\begin{gathered}
\operatorname{DOS}(\omega)=\int_{0}^{\pi} A(\mathbf{k}, \omega) \mathrm{d} k \\
n=\int_{-\infty}^{\mu} \operatorname{DOS}(\omega) \mathrm{d} \omega
\end{gathered}
$$

In the following this relation is used to determine the chemical potential. Starting from an arbitrary upper integration limit $\tilde{\mu}$ one can define $\mu$ by demanding that

$$
\tilde{n}(\tilde{\mu})=\int_{-\infty}^{\tilde{\mu}} \operatorname{DOS}(\omega) \mathrm{d} \omega \stackrel{!}{=} n, \quad \text { for } \tilde{\mu}=\mu .
$$

Figure 3.4 shows spectral function, DOS and integrated DOS for a single 12-site cluster at half-filling and $U / t=4.0$. Within the gap of the spectral function, the integrated DOS stays at $\tilde{n}=1$, which means that two values $\mu_{-}$and $\mu_{+}$characterize the region, where $\tilde{n}(\mu)=n$.

Returning to the electron density $n$, integration of the spectral function gives

$$
\begin{aligned}
n & =\int_{0}^{\pi} \mathrm{d} k \int_{-\infty}^{\mu} \mathrm{d} \omega A(\mathrm{k}, \omega) \\
& =2 \int_{0}^{\pi} \mathrm{d} k \sum_{l}\left|\hat{Q}_{l}(\mathbf{k})\right|^{2}, \quad \omega_{l}<\mu .
\end{aligned}
$$




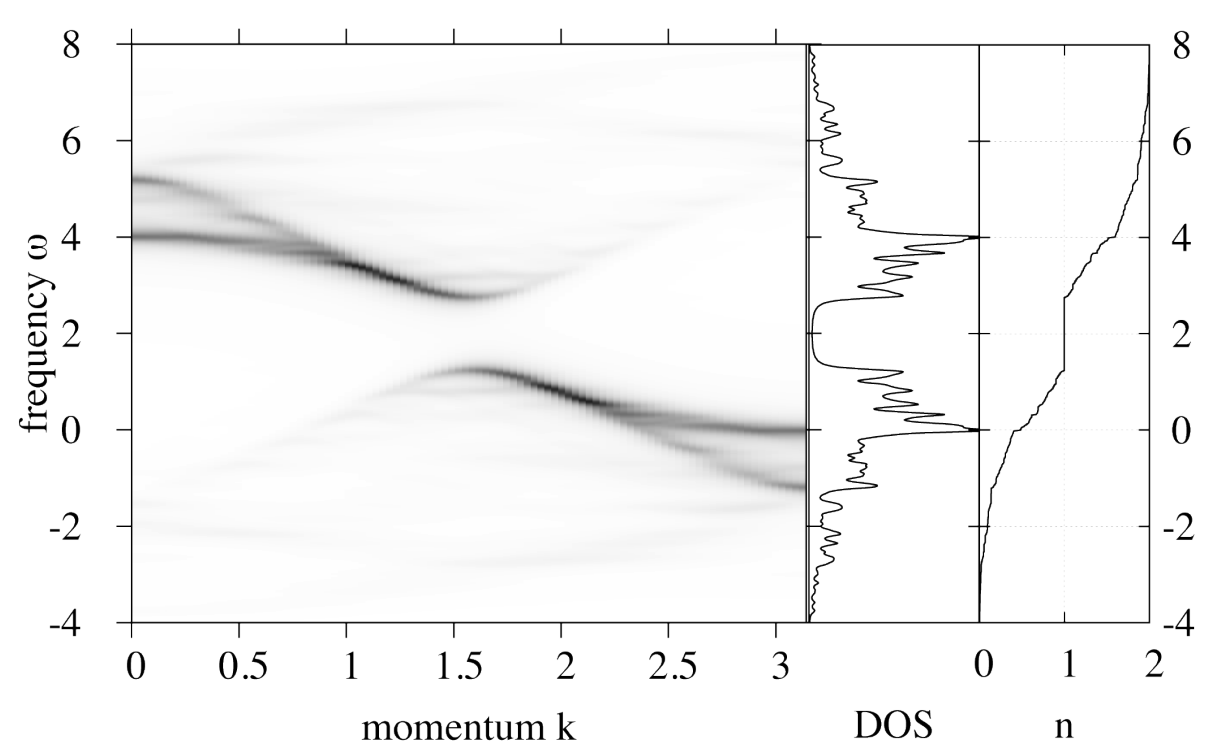

Figure 3.3: Spectral function $A(\mathbf{k}, \omega)$, density of states (DOS) and integrated spectral weight $n$ for a 12 -site cluster with $U / t=4.0$ and cluster filling $n_{c l}=1.0$. The broadening for the spectral function was set to $\eta=0.1$.

In practice, one can replace the integral over $k$ by a sum over $k$ if one treats a limited number of (super-)clusters. This translates into a finite resolution of the density of states and after integration over $\omega$ finally to an approximated electron density $n$, as can be seen in figure 3.4. For most calculations a number of $N_{k}=1000 \mathrm{k}$-points is enough to achieve a sufficient precision of $n$. However, as the deviations from the exact Bethe ansatz solution were found to be largest at intermediate interaction strength [SPP02], the figure shows results for $U / t=4$, where in case of the supercluster consisting of two and three clusters a value of $N_{k}=1000$ already leads to errors of $\Delta(\tilde{\mu} / t) \approx 0.005$.
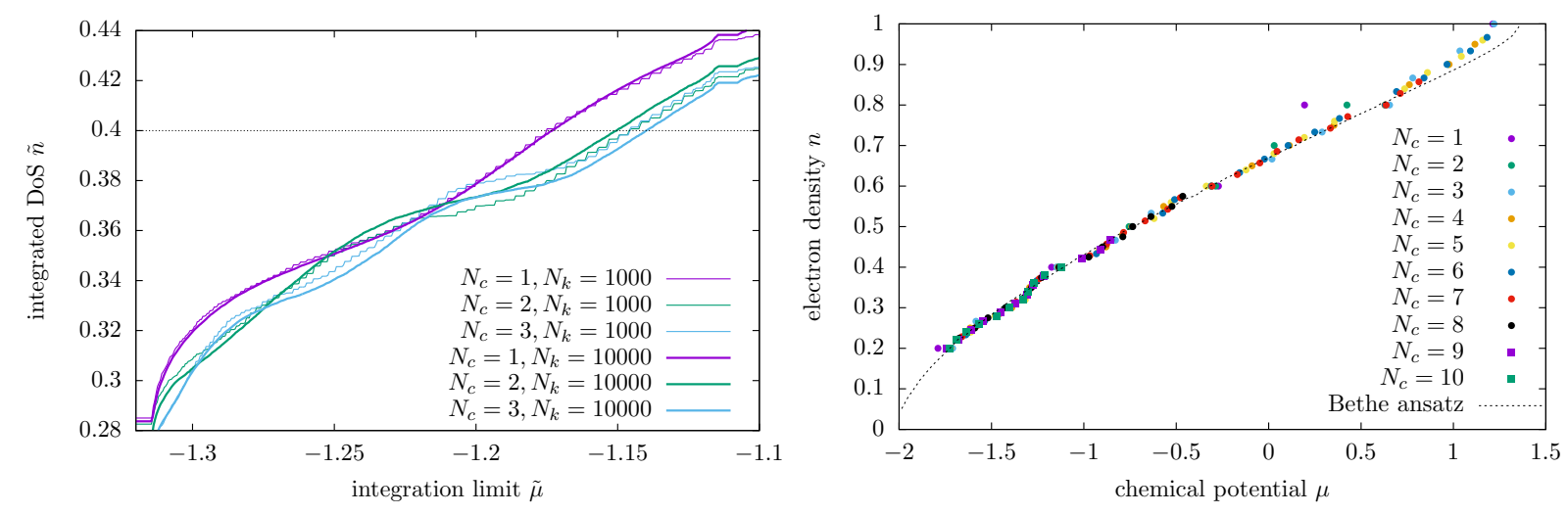

Figure 3.4: Left panel: Integrated density of states $\tilde{n}=\int_{-\infty}^{\tilde{\mu}} \operatorname{DOS}(\omega) \mathrm{d} \omega$ as a function of $\tilde{\mu}$. The chemical potential $\mu$ is determined via comparison of $\tilde{n}$ with the known supercluster density $n_{\mathrm{sc}}=0.4$. Two calculations with 1000 and $10000 k$-points are compared. Right panel: Electron density $n$ as a function of chemical potential $\mu$ for superclusters of $L_{c}=10$ site clusters. The data is compared to the Bethe ansatz solution, which is taken from reference [SPP02 $]^{1}$.

In addition to errors due to $k$-space discretization, finite-size effects might influence the determination of $n$, especially if the value of $\mu$ is e.g. close to a sharp peak of the DOS 
or close to a gapped region of the spectral function.

One possible way of avoiding these imprecisions is to change the cluster size and thereby the spectral function. Another possibility is the use of superclusters, as the supercluster construction also leads to a different spectral function.

In order to arrive at a cluster with total spin $S_{c}^{z}=0$, only clusters with an equal number of up- and down-spin electrons were used. For a 10-site cluster this leads to 11 possible electron densities. Due to particle-hole symmetry of the model, it is sufficient to investigate only half of the $n$-vs.- $\mu$ curve.

The right panel of figure 3.4 shows the electron density $n$ as a function of chemical potential $\mu$ for different superclusters, which all consist of 10-site clusters but differ in the number of clusters that make up the supercluster. Inside the supercluster, clusters with different electron filling can be combined to allow for more electron densities inside the supercluster than the $N_{n, c}$ densities that can be realized within one of the clusters. Thereby it is possible to realize $N_{n, c} \cdot N_{c}$ electron densities within the supercluster. Still, different combinations of cluster densities lead to the same supercluster density. The cluster densities of the superclusters of figure 3.4 were chosen such, that only clusters with two different electron densities constituted the supercluster. For example, for a supercluster with electron density $n=0.25$ consisting of $N_{c}=410$-site clusters, three clusters with electron density $n=0.2$ and one cluster with the next higher electron density of $n=0.4$ were combined into a supercluster.

\section{Treating Spins: Cluster Perturbation Theory for Spin Models $\quad 3.1 .4$}

A spin formulation of cluster perturbation theory was formulated in 2010 by Ovchinnikov et al., who applied it to a dimerized Heisenberg chain [OBS10]. In this model the exchange interaction $J_{i j}$ between nearest neighbor sites alternates. Even bonds have a strength of $J_{1}$, odd bonds a strength of $J_{2} \ll J_{1}$. By choosing a tiling of the infinite system into identical clusters such that inter-cluster interactions are always of strength $J_{2}$, they could motivate to treat these interaction terms within first-order perturbation theory and use spin cluster perturbation theory (SCPT).

For ordinary (an)isotropic Heisenberg chains the treatment of inter-cluster interactions by first order perturbation theory is not well justified, as intra- and inter-cluster interactions have the same strength $J$. Introducing the cluster tiling of the infinite system and using SCPT therefore leads to an error. However, as large cluster sizes can be treated within this method by means of an exact diagonalization solver (cluster sizes of $L>20$ can be used), the ratio of inter-cluster to intra-cluster bonds is small and the resulting additional errors become managable. In contrast to the spin-version of variational cluster approximation that was proposed by Filor and Pruschke [FP10, FP14], the spin cluster perturbation theory considered here works directly at zero temperature.

The idea of SCPT is the same as for standard CPT, which is why it will be described only briefly in the following. A more detailed discussion can be found in the original publication by Ovchinnikov et al. [OBS10]. One splits the system into clusters with

\footnotetext{
${ }^{1}$ Bethe ansatz curve taken from reference [SPP02]. The calculation follows the derivation of Lieb and $W u$ [LW68]; a detailed derivation can also be found in chapter 6 of the book by Essler et al. [EFK $\left.{ }^{+} 05\right]$.
} 
Hamiltonian $\mathcal{H}_{0}$ and an inter-cluster part $V$, therefore $\mathcal{H}=\mathcal{H}_{0}+V$. In contrast to CPT, $V$ contains inter-cluster spin interactions $V=\sum_{i, j} V_{a b}^{i j} S_{i a} S_{j b}$. Based on works by $V a k s$, Larkin and Pikin, who used spin diagram techniques to derive a spin self-energy [VLP67], an equation can be obtained which connects the Green function $G$ with the cluster Green function $G_{0}$ [IS88]:

$$
G^{-1}=G_{0}^{-1}-V
$$

This equation is known as Larkin equation. As the spin operator can be decomposed into a longitudinal and a transversal part, the (cluster) Green function is also split into these components:

$$
G_{i j}^{-+}(\tau)=-\frac{1}{2}\left\langle\left\langle S_{i}^{-}(\tau) S_{j}^{+}(0)\right\rangle\right\rangle, \quad G_{i j}^{z z}(\tau)=-\left\langle\left\langle S_{i}^{z}(\tau) S_{j}^{z}(0)\right\rangle\right\rangle .
$$

In this compact notation the time ordering and thermal average are included as $\langle\langle A(\tau) B(0)\rangle\rangle=$ $\operatorname{Tr}\left\{e^{-\beta H} \hat{T} A(\tau) B(0)\right\} / \operatorname{Tr}\left\{e^{-\beta H}\right\}$. Similar to an electronic Green function these can also be written in Lehmann representation at zero temperature:

$$
\begin{aligned}
G_{i j}^{-+}(\omega) & =\sum_{n} \frac{\left\langle 0\left|S_{i}^{-}\right| n\right\rangle\left\langle n\left|S_{j}^{+}\right| 0\right\rangle}{\omega+i \eta-E_{n}+E_{0}} \\
G_{i j}^{z z}(\omega) & =\sum_{n \neq 0} \frac{\left\langle 0\left|S_{i}^{z}\right| n\right\rangle\left\langle n\left|S_{j}^{z}\right| 0\right\rangle}{\omega+i \eta-E_{n}+E_{0}}
\end{aligned}
$$

However, for $G^{z z}$ it is important to get rid of the ground state contribution as one is only interested in excitations. The remaining weights $\left\langle 0\left|S_{i}^{z}\right| n\right\rangle$ can be calculated as follows:

$$
\begin{aligned}
\left\langle 0\left|S_{i}^{z}\right| n\right\rangle & =\sum_{a, b}\langle 0 \mid a\rangle\langle b \mid 0\rangle\left\langle a\left|S_{i}^{z}\right| b\right\rangle \\
& =\sum_{a, b} f(a)\langle 0 \mid a\rangle\langle b \mid 0\rangle \delta_{a, b}, \quad f(a) \delta_{a, b}= \pm \frac{1}{2}\langle a \mid b\rangle \\
& =\sum_{a} f(a)\langle 0 \mid a\rangle\langle a \mid m\rangle .
\end{aligned}
$$

Once the cluster Green function and the Fourier transformed $V$ matrix $V_{a b}(Q)=\sum_{R} V_{a b}^{0 R} e^{i R Q}$ are set up, the approximated Green function is calculated via the Larkin equation. Using the reperiodization prescription of reference [SPPL00], one periodizes the Green function with respect to the superlattice and obtains

$$
G_{C P T}^{\alpha}(k, \omega)=\frac{1}{L} \sum_{i, j=1}^{L} G_{i j}^{\alpha}(k, \omega) e^{-i k\left(r_{i}-r_{j}\right)}, \quad \alpha \in\{z z,-+\} .
$$

\section{Spin Structure Factor and Magnetization Curves within Spin Cluster Perturbation Theory}

Similar to the evaluation of the spectral function $A(k, \omega)$ for electronic systems, the spin structure factors $S^{z z}$ and $S^{-+}$are obtained from the longitudinal and transversal spin Green function:

$$
S^{\alpha}(k, \omega)=-\frac{1}{\pi} \lim _{\eta \rightarrow 0^{+}} \operatorname{Im} G_{C P T}^{\alpha}(k, \omega+i \eta), \quad \alpha \in\{z z,-+\} .
$$


In order to calculate the magnetization, one usually makes use of sum rules. This can be seen directly in the case of a finite-size chain which can be treated within exact diagonalization:

$$
\begin{aligned}
\frac{1}{\pi} \int_{0}^{\infty} d \omega \frac{1}{L} \sum_{k} S^{-+}(k, \omega)= & \frac{1}{\pi} \int_{0}^{\infty} d \omega \frac{1}{L^{2}} \sum_{k} \sum_{l, m} e^{i k(l-m)} \sum_{n} 2 \pi \delta\left(\omega-E_{n}+E_{0}\right) . \\
& \left\langle\Omega\left|S_{l}^{-}\right| n\right\rangle\left\langle n\left|S_{m}^{+}\right| \Omega\right\rangle \\
& \frac{1}{L} \sum_{l, m} \delta_{l, m} \sum_{n}\left\langle\Omega\left|S_{l}^{-}\right| n\right\rangle\left\langle n\left|S_{m}^{+}\right| \Omega\right\rangle \\
= & \frac{1}{L} \sum_{j}\left\langle\Omega\left|S_{j}^{-} S_{j}^{+}\right| \Omega\right\rangle, \quad S_{j}^{-} S_{j}^{+}=S_{x}^{2}+S_{y}^{2}-i \hbar\left(S_{y} S_{x}-S_{x} S_{y}\right) \\
= & \frac{1}{2}-\left\langle S_{z}\right\rangle
\end{aligned}
$$

Within spin cluster perturbation theory, instead of $G_{0}$ one has to use the CPT-Green function $G_{\mathrm{CPT}}=\sum_{l, m} e^{-i k(l-m)} \frac{G_{0}}{1-V(k) G_{0}}$ and it is far from clear whether the sum rules are still fulfilled. Nevertheless, by taking this equation one can define the approximated magnetization of SCPT as

$$
\left\langle S_{z}\right\rangle_{\mathrm{CPT}}=\frac{1}{2}-\frac{1}{\pi} \int_{0}^{\infty} d \omega \frac{1}{L} \sum_{k} S_{\mathrm{CPT}}^{-+}(k, \omega) .
$$

Using the Band Lanczos algorithm to determine the Green function via the Q-matrix formulation, the last term can be evaluated as

$$
\begin{aligned}
\frac{1}{\pi} \int_{0}^{\infty} d \omega \frac{1}{L} \sum_{k} S_{\mathrm{CPT}}^{-+}(\mathbf{k}, \omega) & =\frac{1}{\pi} \int_{0}^{\infty} d \omega \frac{1}{L} \sum_{k} \lim _{\eta \rightarrow 0^{+}} \sum_{r} \frac{1}{\pi} \frac{\left|\tilde{\mathbf{Q}}_{r}(\mathbf{k})\right|^{2} \eta}{\left(\omega-\tilde{\omega}_{r}(\mathbf{k})\right)^{2}+\eta^{2}} \\
& =\frac{1}{L} \sum_{k} \sum_{r}\left|\tilde{\mathbf{Q}}_{r}(\mathbf{k})\right|^{2} \\
& =\frac{1}{L} \int d \mathbf{k}|\tilde{\mathbf{Q}}(\mathbf{k})|^{2},
\end{aligned}
$$

where $\tilde{\mathbf{Q}}(\mathbf{k})=1 / \sqrt{L} e^{-i \mathbf{k} \cdot \mathbf{R}} \mathbf{Q} \mathbf{U}(\mathbf{k})$ includes both the inter-cluster interactions via $\mathbf{V}(\mathbf{k})$ in $\mathbf{U}(\mathbf{k})$ and the reperiodization factor $1 / \sqrt{L} e^{-i \mathbf{k} \cdot \mathbf{R}}$. As expected for every translation symmetric one-particle expectation value [Sén08a], the obtained magnetization is identical for ED and single-cluster SCPT, see figure 3.5.

Any finite cluster always leads to a discrete set of poles in $S(\omega)$, which is represented in $\tilde{\omega}_{r}$. If one now takes the limit $\eta \rightarrow 0^{+}$and performs the exact frequency integration, one arrives at the last line of the equation shown above. Due to the finite size of the cluster this treatment does not lead to a smooth magnetization curve, but instead to steps in the function (shown in figure 3.5), which merge only in the limit $L_{c} \rightarrow \infty$ to form a continuum.

So far, single cluster SCPT has been described. In the following subsection, it is explained how a supercluster construction can be used to obtain magnetization curves.

\section{Magnetization Curves via Superclusters}

The idea to access more magnetizations by using superclusters than via exact diagonalization goes back to the construction by Sénéchal et al. [SPP02]. In the case of SCPT 

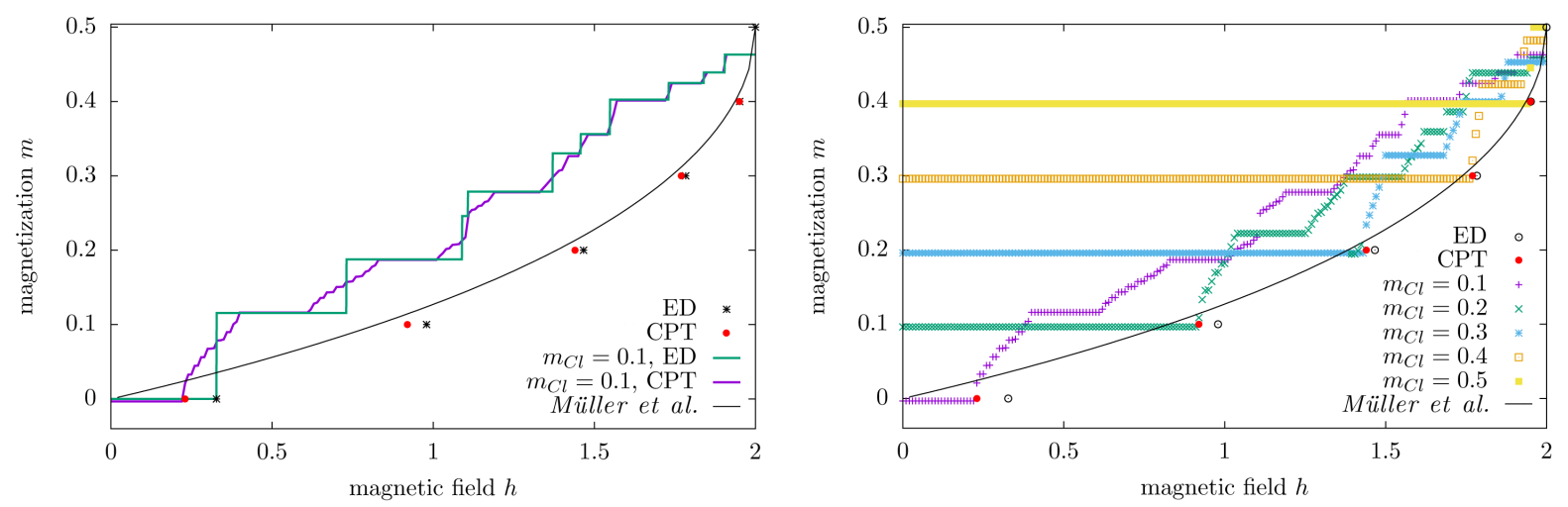

Figure 3.5: Left panel: Comparison of the magnetization as a function of magnetic field strength $h$ of a one-cluster CPT of $L_{c}=10$ sites (red dots) with ED (black dots) shows slight improvement. Right panel: When determining $h$ for each cluster magnetization, one notices that compared to the steps of ED within CPT the steps are often cosine-shaped, what then leads to a shift of $h$ (red vs. black dots). Both panels show results for the isotropic Heisenberg chain with a equidistant $k$-discretization with $N_{k}=200$ points. The solid curve is the approximate magnetization curve according to reference [MTBB81].

this is achieved by combining $M$ clusters of $L$ spins each, but with different magnetizations $m^{i}$ to one supercluster. The inter-cluster interaction is again treated in first order perturbation theory - therefore one uses no additional approximation, but simply takes advantage of the fact that the Green function is block diagonal. This supercluster is then repeated periodically as before.

When using superclusters which consist of clusters of different magnetization, one also has to think about how to take this into account when constructing the $\mathrm{V}$ matrix. Usually the $\mathbf{V}=\mathbf{t}-\mathbf{t}^{\prime}$ contains all differences in one-body terms between original and clustered system. When considering spin-spin interaction terms between clusters or superclusters, this is not much different to the treatment without superclusters. In addition, the difference in magnetization shows up in the diagonal terms, as they can be connected with an effective magnetic field in the $\mathrm{z}$ direction.

The magnetization of each individual cluster is set at the beginning and would necessitate a certain yet to be determined magnetic field $h$. This magnetic field is now rewritten in terms of $S^{-}$and $S^{+}$operators, which then enter in the $V$-matrices:

$$
\begin{aligned}
h S_{i}^{z} & =-i h\left[S_{i}^{x}, S_{i}^{y}\right]=S_{i}^{+} S_{i}^{-}-\left(S_{i}^{x}\right)^{2}-\left(S_{i}^{y}\right)^{2} \\
& =h\left(S_{i}^{+} S_{i}^{-}-\frac{1}{2}\right)=h\left(\frac{1}{2}-S_{i}^{-} S_{i}^{+}\right) .
\end{aligned}
$$


This means, that $\pm h$ enters the $V^{ \pm \mp}$ matrix on the diagonal. In case of a 2-cluster supercluster, the $V$ matrix looks like this:

$$
V^{-+}=\left[\begin{array}{cccc|cccc}
-h & 0 & \ldots & 0 & 0 & \ldots & 0 & J e^{-i L_{s c} k} \\
0 & \ddots & & \vdots & \vdots & & 0 & 0 \\
\vdots & & \ddots & 0 & 0 & 0 & & \vdots \\
0 & \ldots & 0 & -h & J & 0 & \ldots & 0 \\
\hline 0 & \ldots & 0 & J & -h & 0 & \ldots & 0 \\
\vdots & & 0 & 0 & & \ddots & & \vdots \\
0 & 0 & & \vdots & & & \ddots & 0 \\
J e^{i L_{s c} k} & 0 & \ldots & 0 & 0 & \ldots & 0 & -h
\end{array}\right] .
$$

For exact diagonalization the easiest way of obtaining the magnetization curve is to calculate the energy as a function of external magnetic field and determine the crossings between quantum sectors of different magnetization. However, here this is not easily possible as one works with an approximated Green function and not directly with ground state energies, so one has to find another way to determine the dependence $m(h)$. Instead, $h(m)$ is calculated, meaning that the supercluster magnetization

$$
m_{\text {supercluster }}=\frac{1}{M} \sum_{i=1}^{M} m_{\mathrm{CPT}}^{i}
$$

is fixed and the external magnetic field $h$ that is consistent with this is determined. This can be achieved by demanding

$$
m_{\text {supercluster }} \stackrel{!}{=} \frac{1}{2}-\frac{1}{\pi} \int_{h}^{\infty} \mathrm{d} \omega \frac{1}{N_{k}} \sum_{k} S_{\text {supercluster }}^{-+}(\mathbf{k}, \omega) .
$$

In order to determine $h$ for large superclusters, it can be useful to keep a small but finite $\eta$ to broaden the steps in $m$. Instead of an exact frequency integration one then turns to integrating the spin structure factor $S_{\text {supercluster }}^{-+}(k, \omega)$ over $k$ and $\omega$ numerically. This seems strange since an exact analytical treatment should usually be preferred to an approximate numerical treatment. Yet it turns out to have some advantages for the computational calculation. To see this one has to keep in mind, that the $\tilde{Q}$ matrix is of size $N \times R$, where $N=M \cdot L$ and $R=\sum_{i=1}^{M} R_{i}$ with Lanczos iteration numbers $R_{i}$ of typical size $R_{i} \sim 100-200$. Hence, each sub-matrix $Q_{i}$ is not much of a problem, but the resulting supercluster $\tilde{Q}$ matrix can become large and then leads to costly matrix manipulations.

By using numerical frequency integration and a finite artificial broadening $\eta$, one can calculate the Green function matrices $G_{i j}(\omega)$ for each frequency in the integration and set up the supercluster $G(\omega)$, which is now "only" a $N \times N$ matrix. For the $k$-sum one is left with matrix operations with this much smaller matrix size. Especially for superclusters which consist of many clusters, this seems to be necessary in order to keep the numerical cost manageable.

Another approach to combine clusters with different magnetization into one supercluster consists in shifting the energies of the cluster Green functions "by hand". As the individual 
cluster magnetization usually differs from the supercluster magnetization $m_{\mathrm{sc}}$, the energy difference through the applied magnetic field amounts to $h \cdot L_{c} \cdot\left(m_{\mathrm{sc}}-m_{c}\right)$. When taking this energy difference into account the magnetic field does not enter in the $\mathrm{V}$ matrix and the lower integration threshold in equation (3.1) should be again equal to zero. Still, the magnetic field strength $h$ has to be chosen in such a way that the equation is fulfilled. This second way of accounting for the inhomogeneous cluster magnetizations within a supercluster is also tested in the following subsection. Both ways of constructing a supercluster will lead to different results for the magnetization curve. This has to do with the fact that the "full" spin operator is split into the transversal and longitudinal part. Including a field in $S^{z}$ direction in the calculation of the spin structure factor $S^{-+}(k, \omega)$ is therefore different from electronic CPT where a chemical potential $\mu$ enters the (cluster) Green function directly.

\section{Spin Structure Factor and Magnetization Curve of the Heisenberg Chain}

Here, only some selected results for the spin structure factor and the magnetization curve are shown for the isotropic Heisenberg chain. This prototypical quantum spin model can be solved exactly using Bethe ansatz [Bet31]. Using this technique, magnetization curves [MTBB81] and even spectral functions [KMCvdB11, CKSW12] are accessible, which make it a good test case for SCPT.

For the plots of the spectral function the $k$-space has been discretized equidistantly with $N=200 \mathrm{k}$-points and an artificial broadening of $\eta=0.02$ is used. More results for the spin structure factor, i.a. for the anisotropic Heisenberg chain and the $J_{1^{-}} J_{2}$ Heisenberg ladder, are shown in the appendix, see B.
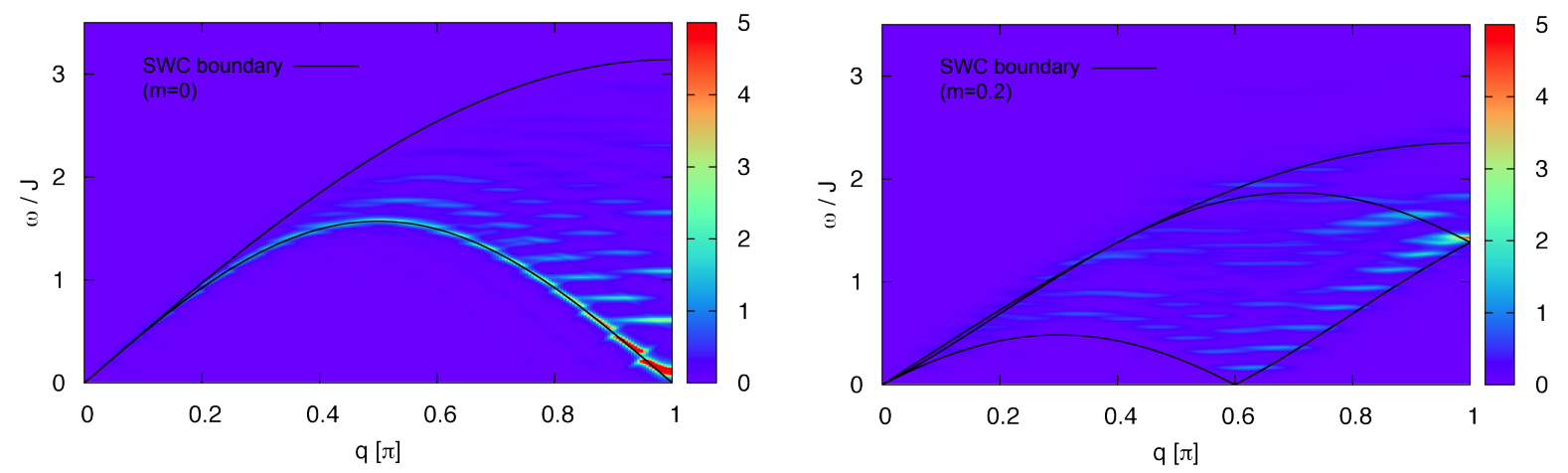

Figure 3.6: Spin structure factor $S^{z z}(q, \omega)$ using a 20-site cluster. The external magnetic field $h / J$ corresponds to magnetizations of $m=0$ (left panel) and $m=0.20$. For comparison, the limits of the spin wave continua contributing to $S^{z z}$ of an infinite Heisenberg chain are shown as obtained in reference [MTBB81].

In reference [MTBB81], Müller et al. derived upper and lower boundaries of the two spin wave continua (SWC) which they identified to contribute to the spin structure factor $S^{z z}$ of an infinite Heisenberg chain ${ }^{2}$. These limits are plotted for comparison in figure 3.6. As can be seen from the figure most of the weight of the spin structure factor stays within the SWC boundaries in the case of zero magnetic field. The qualitative features with respect to the relative positions of the poles of the spin structure factor and the

\footnotetext{
${ }^{2}$ More details on the spin wave continua of reference [MTBB81] are explained in the appendix, see B
} 
redistribution of weight for increasing magnetic field are well reproduced when comparing to Bethe ansatz spectra from reference [CKSW12]. However, when plotting the spectra logarithmically it is clearly visible that the structure factor shows small but finite weight within the forbidden region $\omega / J<\epsilon_{1 L}$ for $h=0$ (and $\omega / J<\epsilon_{2 L}$ for $h \neq 0$ ). This is due to the reperiodization of the cluster spin Green function that is necessary within SCPT. For larger clusters, the weight in this forbidden region becomes smaller, but still exists. To make the contributions of the $L_{c}$ different accessible $k$-points within the cluster visible, the broadening has been chosen rather small $(\eta=0.02)$. When increasing the broadening, the contributions smear out and form a more or less continuous spectrum.

For higher values of the magnetization $m$, deviations of the lowest excitations with considerable weight and the lower boundary of SWC become apparent. The fact that the lower boundary is not reached between $0<q<q_{0}$ is a phenomenon which has been encountered before in ED and is typical for finite-size chains [dCP62, MTBB81]. In fact, as the first SWC with higher excitations is negligible in the limit $L_{c} \rightarrow \infty$, one would expect that the spectrum lies within the boundaries of the second SWC when the cluster size becomes large.

When applying a magnetic field, an approximate formula for the magnetization curve was derived in reference [MTBB81] based on Bethe ansatz calculations. For a given magnetic field $h$, the magnetization $m=S_{\text {total }}^{z} / L$ is then given approximatively as

$$
m(h)=\frac{1}{\pi} \arcsin \frac{1}{1-\pi / 2+\pi / h}, \quad 0 \leq h \leq 2 .
$$
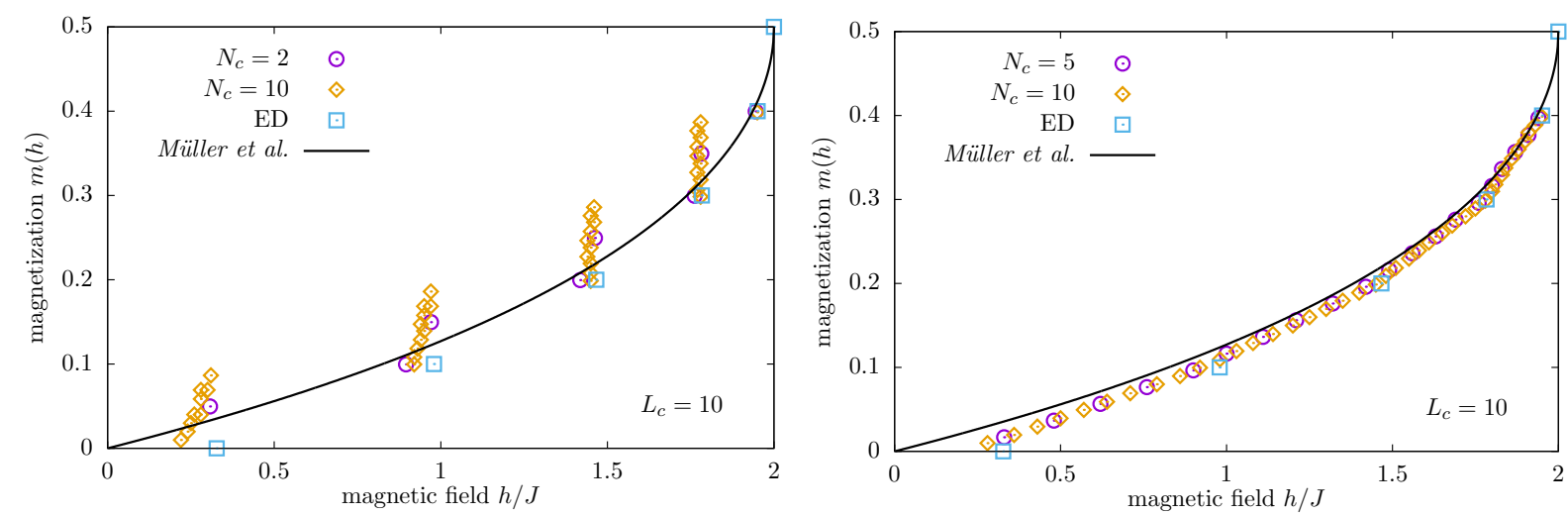

Figure 3.7: Magnetization as a function of external magnetic field $h / J$. The data was obtained by using superclusters consisting of $N_{c} 10$-site clusters. In the left panel, results from the first supercluster construction described in the text are shown, the right panel shows data for the second construction scheme. The solid curve is the approximate magnetization curve according to reference [MTBB81].

Figure 3.7 shows the magnetization curve obtained by SCPT for both ways of constructing a supercluster compared to the ED result. In contrast to standard cluster perturbation theory, using a supercluster construction does not improve the results much.

In the left panel, the way of constructing a supercluster presented first in the previous 
section has been used. The number of viable supercluster magnetizations is enhanced by a factor $N_{c}$ compared to single-cluster SCPT. However, the magnetic fields that were determined via equation (3.1) lead to only minor changes between the magnetizations that can be realized within a single cluster. At large magnetic fields, where the magnetization curve becomes quite steep, it is also difficult to achieve magnetic fields with reasonable precision to resolve this slope.

When using the second proposed scheme for constructing a supercluster, the magnetization curve seems to be much improved at first sight. The number of viable magnetizations is of course unchanged, but the magnetic field strengths form a seemingly smooth curve and do not lead to a step-like shape as expected from ED. Changing the number of clusters inside the supercluster reveals the somewhat artificial nature of its construction. Between magnetizations that can be realized on a single cluster, the supercluster construction leads to a nearly linear interpolation only. This can be explained by the way it is generated the cluster Green functions are shifted with respect to the average supercluster magnetization.

In this sense, no new information on the magnetization curve is gained from the supercluster construction, but it allows to access additional magnetization values via SCPT.

In the previous subsection a spin formulation of cluster perturbation theory has been introduced and some characteristic results for the isotropic Heisenberg chain have been presented. By investigating exactly solvable models like the isotropic and anisotropic (see appendix B) Heisenberg chain, SCPT was shown to be a computationally efficient tool to visualize the spin structure factor. Even the frustrated $J_{1^{-}} J_{2^{-}}$Heisenberg ladder has been successfully treated within spin cluster perturbation theory, see appendix B.

However, compared to techniques like exact diagonalization which have comparable computational cost, this (non-variational) cluster technique does not improve the results significantly. Instead, SCPT could be considered to be a physically motivated interpolation scheme between the available ED data points. Especially when adding an artificial broadening $\eta$, smooth spin structure spectra can be obtained.

As the number of accessible magnetizations is limited to the number of cluster sites $L_{c}+1$, a supercluster construction that proved to be useful in standard CPT was tested. The supercluster construction in standard CPT was revisited and applied for the first time to SCPT. Unfortunately, the supercluster construction lead only to minor improvements on the magnetization curve compared to exact diagonalization.

Spin CPT can be considered to fill the gap between exact diagonalization and more elaborate, but limited techniques like the spin VCA proposed in [FP14]. However, this approach to treat spin systems will not be further pursued within this thesis. Instead, the variational cluster approximation is introduced in the next section and afterwards used for the investigation of the Hubbard and Kondo lattice model. 


\section{Self-Energy Functional Theory and the Variational Cluster Approximation}

In the following section the self-energy functional theory (SFT) developed by Potthoff [Pot03a, Pot03b] is introduced. Subsequently the variational cluster approximation is introduced which is based on SFT. When including bath sites that are coupled to the cluster sites, variational cluster approximation is sometimes called cluster dynamical impurity approximation (CDIA) [Pot05] and the single-site version where the reference system consists of one interacting site coupled to a non-interacting bath site is called dynamical impurity approximation (DIA) [Pot03b]. Several conceptional and technical details of the technique and its implementation are presented. The following subsections on the self-energy functional and the variational cluster approximation are based on references [Pot03a, Pot03b, Sén08a, Pot11, Pot14].

\section{Self-Energy Functional Theory}

For the derivation of Potthoff's self-energy functional theory (SFT), one assumes a system with local interactions

$$
\mathcal{H}=\sum_{\alpha, \beta} t_{\alpha \beta} c_{\alpha}^{\dagger} c_{\beta}+\sum_{\alpha, \beta, \gamma, \delta} U_{\alpha \beta \gamma \delta} c_{\alpha}^{\dagger} c_{\beta}^{\dagger} c_{\gamma} c_{\delta}
$$

where $\alpha, \beta, \gamma, \delta$ can be composite indices consisting of spin, orbital and local degrees of freedom. Considering further more that the system is in thermal equilibrium, one can describe it by looking at the elementary one-body excitations. The natural quantity for this is the causal Green function at temperature $T$ and chemical potential $\mu$, which is defined via

$$
G_{\alpha \beta}(i \omega)=\left\langle\left\langle c_{\alpha} ; c_{\beta}^{\dagger}\right\rangle\right\rangle
$$

with the fermionic Matsubara frequencies $i \omega=i(2 n+1) T \pi, n \in \mathbb{N}$. All effects of higher order excitations, which can be obtained by repeatedly applying the equation of motion to the Green function to arrive at an interleaved series of many-body Green functions, are collected in the self-energy $\boldsymbol{\Sigma}$. The self-energy thereby connects the non-interacting Green function $\mathbf{G}_{\mathbf{0}}{ }^{-1}=[i \omega+\mu-\mathbf{t}]$ with the full one-body Green function $\mathbf{G}$ via the Dyson equation

$$
\mathrm{G}=\mathrm{G}_{\mathbf{0}}+\mathrm{G}_{\mathbf{0}} \boldsymbol{\Sigma} \mathrm{G} \text { or } \boldsymbol{\Sigma}=\mathrm{G}_{\mathbf{0}}{ }^{-1}-\mathrm{G}^{-1} \text {. }
$$

At thermodynamic equilibrium, the system is described by a grand canonical ensemble with the grand canonical potential $\Omega$. Luttinger and Ward showed [LW60] that the grand potential can be expressed by a functional that includes the Green function and a summation of skeleton diagrams

$$
\Omega^{\mathrm{BK}}=\Phi^{\mathrm{LW}}+\operatorname{Tr} \ln (-\mathbf{G})-\operatorname{Tr}\left(\left(\mathbf{G}_{\mathbf{0}}{ }^{-1}-\mathbf{G}^{-1}\right) \mathbf{G}\right) .
$$

It is referred to as Baym-Kadanoff functional following a paper by Baym and Kadanoff in 1961 [BK61], who approximated $\Omega^{\mathrm{BK}}$ with exactly known, but approximate functionals to obtain so-called conserving approximations. The trace $\operatorname{Tr}$ of an observable $O$ should be read as $\operatorname{Tr} \mathbf{O}=T \sum_{\alpha, \omega} O_{\alpha \alpha}(i \omega)$ and the first term of the equation denotes the so-called 
Luttinger-Ward functional $\Phi^{\mathrm{LW}}$. This functional, which was introduced by Luttinger and Ward already in 1960 [LW60], is the sum of all two-particle irreducible Feynman diagrams, sometimes called skeleton diagrams, shown in figure 3.8. Two-particle irreducible means, that each of the diagrams cannot be separated into two distinct parts by cutting two fermionic lines. The first two diagrams are the Hartree diagrams.

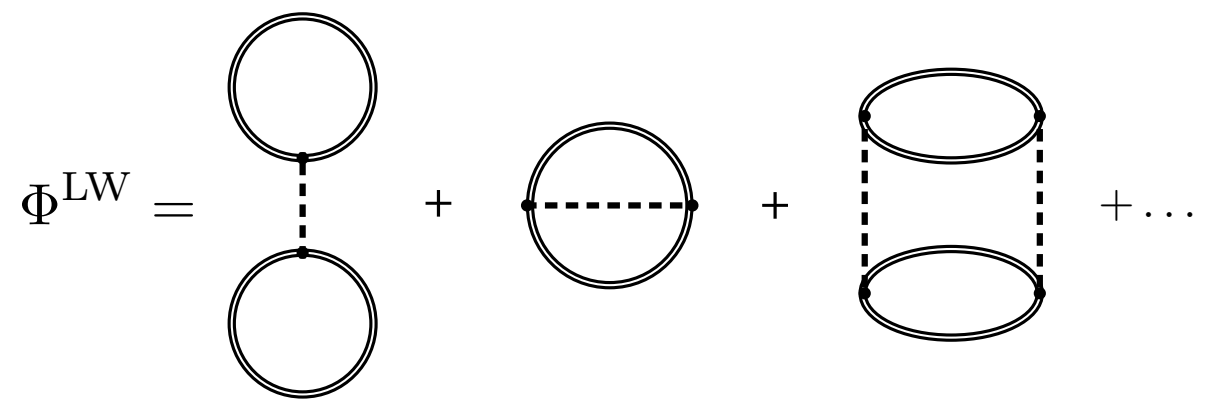

Figure 3.8: Definition of the Luttinger Ward functional $\Phi^{\mathrm{LW}}[\mathbf{G}, U]$ as the sum of all twoparticle irreducible Feynman diagrams. Double lines denote the interacting Green function $\mathbf{G}$ and the dashed lines the interaction $U$.

However, Potthoff showed in 2006 that the Luttinger-Ward functional can be derived within a functional-integral approach which does not rely on the skeleton-diagram expansion and is not perturbative [Pot06a].

If one cuts one fermionic line in the Luttinger-Ward functional, which corresponds to performing the functional derivative with respect to the Green function, one ends up with the diagrammatic series for the self-energy, which means that

$$
T^{-1} \frac{\delta \Phi^{\mathrm{LW}}[\mathbf{G}, U]}{\delta \mathbf{G}}=\Sigma[\mathbf{G}]
$$

Therefore, the Baym-Kadanoff functional can be used to derive the Green function $\mathbf{G}$ via a variational principle as

$$
\frac{\delta \Omega^{\mathrm{BK}}[\mathbf{G}, U]}{\delta \mathbf{G}}=T\left(\boldsymbol{\Sigma}-\mathbf{G}^{-1}-\mathbf{G}_{\mathbf{0}}^{-1}\right)=0
$$

At this point, it is necessary to do approximations as the Luttinger-Ward functional $\Phi^{\mathrm{LW}}$ cannot be calculated exactly for non-trivial systems. One possibility is to replace the Luttinger-Ward functional by an approximate functional that can be calculated, e.g. a sub-set of diagrams. Approximations that are obtained in this way are called $\Phi$-derivable, conserving [BK61, Bay62], or type-II approximations [Pot05] respectively. Examples are Hartree and Hartree-Fock approximations [BK61] and the fluctuation exchange (FLEX) approximation [BSW89]. Other possibilities for approximations can be grouped into two different categories [Pot05]. One category includes type-I approximations, where the Euler equation $\frac{\delta \Omega[\mathbf{G}]}{\delta \mathbf{G}}=\mathbf{\Sigma}-\mathbf{G}_{\mathbf{0}}{ }^{-1}+\mathbf{G}=0$ is approximated, e.g. by inserting the self-energy of the atomic limit as done by Hubbard in 1963 [Hub63]. By approximating the Euler equation one loses the connection to the conserving $\Phi$-functional. Hence, type-I approximations may lead to thermodynamical inconsistencies. The third category are so-called type-III approximations, where the full functional is used but the variational space is drastically reduced. This is the route that will be pursued in the next section within the variational 
cluster approximation.

However, the approximation is not applied to the Baym-Kadanoff functional directly, but to its Legendre transformed version, the Potthoff or self-energy functional (SEF). The reason is that the self-energy can be assumed to be more localized than the Green function, which holds not only in infinite dimensions, where it is found to be local and can be used to formulate DMFT [MV89, MH89], but also at finite dimensions [SC90, SC91]. In order to set up a functional based on the self-energy, one assumes that the relation between Green function and self-energy can be inverted such that $\boldsymbol{\Sigma}=\boldsymbol{\Sigma}[\mathbf{G}]$. This step has been criticized recently by Kozik et al. who showed that the self-energy calculated with diagrammatic Monte Carlo is not a single-valued functional of the Green function and that additional unphysical solutions might be caused by choosing the wrong branch of $\Sigma$ [KFG15]. In a comment on this paper Eder pointed out, that by using the analytical properties of the Green function and the self-energy one can show that there is a unique relation between $\mathbf{G}$ and $\boldsymbol{\Sigma}$ [Ede14]. Nevertheless, computing the self-energy numerically may lead to additional unphysical solutions. This is also the case for VCA, where artificial unphysical solutions have been reported when too many parameters were varied [Ede15]. Assuming the local invertibility of the Green function, one Legendre transforms the BaymKadanoff functional:

$$
\begin{aligned}
\Omega[\Sigma] & =\Phi^{\mathrm{LW}}[\mathbf{G}[\Sigma]]+\operatorname{Tr} \ln \left(-\left(\mathbf{G}_{\mathbf{0}}{ }^{-1}-\mathbf{\Sigma}\right)^{-1}\right)-\operatorname{Tr}(\boldsymbol{\Sigma} \mathbf{G}[\Sigma]) \\
& =F[\boldsymbol{\Sigma}]-\operatorname{Tr} \ln \left(\boldsymbol{\Sigma}-\mathbf{G}_{\mathbf{0}}{ }^{-1}\right),
\end{aligned}
$$

where $F[\Sigma]$ denotes the Legendre transformed Luttinger-Ward functional. Its derivative with respect to $\Sigma$ returns the Green function

$$
T^{-1} \frac{\delta F[\Sigma]}{\delta \Sigma}=T^{-1} \frac{\delta \Phi[\Sigma]}{\delta G} \frac{\delta G}{\delta \Sigma}-\mathbf{G}-\Sigma \frac{\delta \mathbf{G}}{\delta \Sigma}=-\mathbf{G} .
$$

Therefore

$$
\frac{\delta \Omega[\Sigma]}{\delta \Sigma}=T\left(-\mathbf{G}-\left(\boldsymbol{\Sigma}-\mathbf{G}_{\mathbf{0}}^{-1}\right)^{-1}=0\right.
$$

Up to this point, it was always assumed that the exact/physical Green function and selfenergy are used. The idea is now to use the stationarity condition of the self-energy functional to construct approximate self-energies $\Sigma$ in order to approach the system. Therefore, one chooses a reference system $\mathcal{H}^{\prime}=\mathcal{H}_{U}+\mathcal{H}_{\mathbf{t}^{\prime}}$ which has the same interaction term $\mathcal{H}_{U}$ as the original system and uses this reference system to provide trial self-energies $\Sigma^{\prime}$ for the original problem. First of all, one constructs the self-energy functional for the reference system, denoted here by $\Omega^{\prime}\left[\Sigma^{\prime}\right]$ :

$$
\Omega^{\prime}\left[\Sigma^{\prime}\right]=F^{\prime}\left[\Sigma^{\prime}\right]-\operatorname{Tr} \ln \left(\boldsymbol{\Sigma}^{\prime}-\mathbf{G}_{\mathbf{0}}^{\prime-1}\right) .
$$

At this point it is worth taking a look at the functional dependencies of the Legendre transformed Luttinger-Ward functional. It only depends on the interaction $U$ and the interacting Green function $\mathbf{G}$ and not explicitly on the one-body parameters (which could have shown up in form of $\mathbf{G}_{\mathbf{0}}$ ). Due to this universality of the Luttinger-Ward functional one can rewrite it as $\Phi_{U}^{\mathrm{LW}}$. As the reference system was chosen to have the same interaction terms as the original system, its (Legendre transformed) Luttinger-Ward functional is identical to the one of the original system when evaluated at the same self-energy:

$$
\Omega^{\prime}\left[\Sigma^{\prime}\right]+\operatorname{Tr} \ln \left(\Sigma^{\prime}-G_{0}^{\prime-1}\right)=F^{\prime}\left[\Sigma^{\prime}\right]=F\left[\Sigma^{\prime}\right]=\Omega\left[\Sigma^{\prime}\right]+\operatorname{Tr} \ln \left(\Sigma^{\prime}-G_{0}^{-1}\right) .
$$


It is therefore possible to rewrite the self-energy functional with help of the reference system:

$$
\Omega\left[\Sigma^{\prime}\right]=\Omega^{\prime}\left[\Sigma^{\prime}\right]+\operatorname{Tr} \ln \left(\Sigma^{\prime}-G_{0}^{-1}\right)-\operatorname{Tr} \ln \left(\Sigma^{\prime}-G_{0}^{\prime-1}\right) .
$$

The reference system differs from the original system in its one-body parameters $\mathbf{t}^{\prime}$ which can be used to parametrize the self-energies of the reference system: $\Sigma^{\prime}=\Sigma^{\prime}\left(\mathbf{t}^{\prime}\right)$. To fulfill the stationarity condition of the self-energy functional of the reference system one has to vary these one-body parameters to find a stationary solution:

$$
\frac{\delta \Omega[\Sigma]}{\delta \mathbf{t}^{\prime}}=\frac{\delta \Omega[\Sigma]}{\delta \Sigma} \frac{\delta \Sigma}{\delta \mathbf{t}^{\prime}}=0 .
$$

So far, there have been no approximations and the theory is still exact. As briefly discussed for the case of the Baym-Kadanoff functional, it is necessary to do approximations at this point. This leads to the variational cluster approximation.

\section{The Variational Cluster Approximation}

If one would not restrict the space of self-energies and allow all possible reference systems, this theory would be exact. At this point, a Hubbard-III approximation is done which amounts to restricting the variational space to a subset of self-energies.

In case of the variational cluster approximation (VCA) the reference system is chosen to be a clustered version of the original system, some examples are shown in figure 3.9. By dividing the lattice into a superlattice consisting of small clusters, the reference systems lacks some of the hopping terms of the original system. These missing terms can be formally collected into a matrix $\mathbf{V}=\mathbf{t}-\mathbf{t}^{\prime}$. Within this notation, the Hamiltonian of the original system can be rewritten as $\mathcal{H}=\mathcal{H}^{\prime}+\mathbf{V}$. When considering again the self-energies of the reference system, this choice of a certain cluster geometry and cluster size is already an approximation: The self-energies $\Sigma^{\prime}$ have been restricted to those which are representable on one of the small clusters. This sometimes goes by the name of $\mathbf{t}^{\prime}-$ representability [Pot03a].
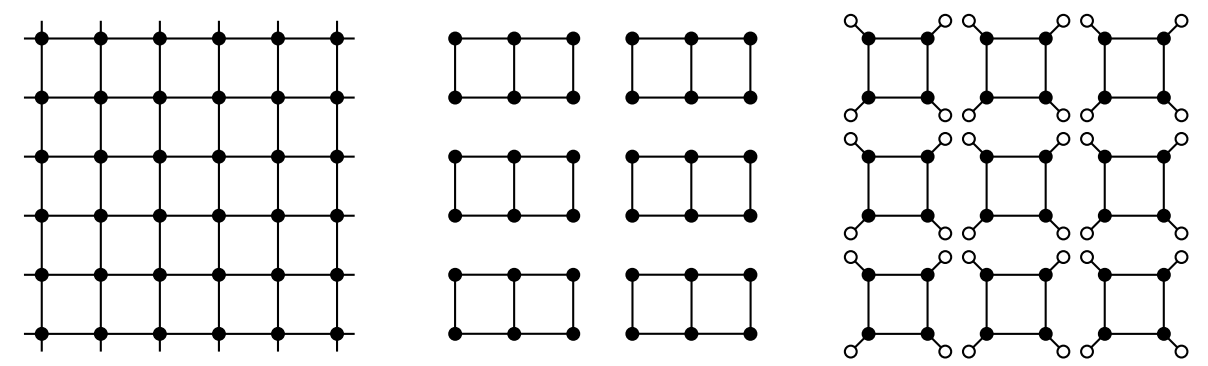

Figure 3.9: Tiling of a square lattice (left panel) into identical $3 \times 2$ clusters (center panel) and into $2 \times 2$ plaquette clusters with one additional (non-interacting) bath site per correlated site (right panel). In both lattice tilings the (local) interaction terms of the tiled system are the same as the ones of the original lattice system.

Hopping terms are just one possible choice for one-body terms in which original and reference system are allowed to differ. One of the big advantages of VCA is its ability to treat 
phases with broken symmetries by adding so-called Weiss fields to the reference system $\left[\mathrm{DAH}^{+} 04\right]$. Some specific choices of additional Weiss field terms will be discussed later. Before, one should note that the space of $t^{\prime}$-representable self-energies is still too large and one has to do an additional approximation to drastically reduce the set of variational parameters. In order to distinguish this sub-set of one-body parameters from the full set of possible one-body terms $\mathbf{t}^{\prime}$ of the reference system, from now the set of variational parameters will be denoted by $\xi^{\prime}$. The prime ( $\left.I\right)$ is simply added for consistency as these are parameters of the reference system. One should, however, note that there are no variational parameters in the original system.

At this point it is also interesting to draw a comparison to the cluster perturbation theory discussed in subsection 3.1.3, which can be regarded as a precursor of VCA. It does not involve a variational principle at all, but also contains the idea of partitioning the lattice into clusters which provide an expansion for the self-energy.

In subsection 3.1.3 the CPT Green function was rewritten in compound indices of the cluster and the superlattice. In this mixed representation it reads

$$
\mathbf{G}^{-1}(\tilde{\mathbf{k}}, \omega)=\mathbf{G}_{\mathbf{0}}{ }^{-1}(\omega)-\mathbf{V}(\tilde{\mathbf{k}})
$$

Comparing this with the definition of the self-energy $\boldsymbol{\Sigma}=\mathbf{G}_{\mathbf{0}}{ }^{-1}-\mathbf{G}^{-1}$ leads to the equality

$$
\mathrm{G}_{0}^{-1}-\mathbf{\Sigma}=\mathrm{G}^{-1}-\mathrm{V}
$$

This very equation can be used to write the expression of equation (3.2) even more compact:

$$
\begin{aligned}
\Omega\left[\boldsymbol{\Sigma}^{\prime}\right] & =\Omega^{\prime}\left[\boldsymbol{\Sigma}^{\prime}\right]+\operatorname{Tr} \ln \left(\boldsymbol{\Sigma}^{\prime}-\mathbf{G}_{\mathbf{0}}^{-\mathbf{1}}\right)-\operatorname{Tr} \ln \left(-\mathbf{G}^{\prime}\right) \\
& =\Omega^{\prime}\left[\boldsymbol{\Sigma}^{\prime}\right]+\operatorname{Tr} \ln \left(\mathbf{V}-\mathbf{G}^{\prime-1}\right)-\operatorname{Tr} \ln \left(-\mathbf{G}^{\prime}\right) \\
& =\Omega^{\prime}\left[\boldsymbol{\Sigma}^{\prime}\right]-\operatorname{Tr} \ln \left(\mathbf{1}-\mathbf{V G}^{\prime}\right) \\
& =\Omega^{\prime}\left[\boldsymbol{\Sigma}^{\prime}\right]-T \sum_{\omega_{n}} \sum_{\tilde{\mathbf{k}}} e^{i \omega_{n} 0^{+}} \ln \operatorname{det}\left(\mathbf{1}-\mathbf{V}(\tilde{\mathbf{k}}) \mathbf{G}^{\prime}(\omega)\right) .
\end{aligned}
$$

Here, the identity $\operatorname{Tr} \ln =\ln$ det has been used in the last step. The first term reduces simply to the ground-state energy $E_{0}$ at zero temperature and the second term can be evaluated purely numerically or semi-analytically, where the sum over Matsubara frequencies is calculated analytically. Before discussing further calculations of the self-energy functional, the $\mathbf{V}$ matrix is inspected and different Weiss fields are discussed.

\section{Weiss Fields}

One of the deficiencies of cluster techniques such as the cluster perturbation theory is their incapability to treat phases with broken symmetries properly. Phases such as magnetically long-range ordered or superconducting phases are simply not within reach. Inspired by the mean-field treatment of ferromagnetism by Weiss [Wei07], the variational cluster approximation allows for the study of these phases by using Weiss fields. Nevertheless, the VCA treatment should not be confused with such a static mean-field theory.

Any additional one-body term can be added to the reference system, but in order to ensure thermodynamical stability, its strength should be included in the set of variational 
parameters $\xi^{\prime}$, see subsection 3.2.2. In case of magnetic ordering, the Weiss field can be written generally as

$$
\mathcal{H}_{\mathrm{M}}=M \sum_{i} e^{i \mathbf{r}_{\mathbf{i}} \cdot \mathbf{Q}}\left(\hat{n}_{i \uparrow}-\hat{n}_{i \downarrow}\right)
$$

where $\hat{n}_{i \sigma}$ is the density operator for electrons with spin $\sigma$ on site $i$. The magnetic ordering vector $\mathbf{Q}$ determines the kind of magnetic ordering: For example, $\mathbf{Q}=(\pi, \pi)$ amounts to (Néel-type) antiferromagnetic and $\mathbf{Q}=(0,0)$ to ferromagnetic order. Choosing $\mathbf{Q}=(\pi, 0)$ would correspond to a staggered order in $x$-direction and ferromagnetic ordering in $y$ direction which in the end amounts to a stripe geometry in $x$-direction. It is important to note at this point, that $M$ is determined via the variational principle and in general different from the expectation value $\langle m\rangle$ of the corresponding magnetic ordering. This is a major difference to static mean-field theory, where $M$ denotes the strength of the molecular magnetic field (mean-field) acting on site $i$ and hence $M=\langle m\rangle$. Still, when one considers length scales that exceed the cluster size, VCA could be viewed as a mean-field approach and some typical artifacts of mean-field theory might become visible [DAH $\left.{ }^{+} 04\right]$. In order to identify and hopefully reduce such artifacts, increasing the cluster size is a typical procedure.

Even superconductivity can be included within VCA by introducing a proper Weiss field. The pairing field in general has the following form:

$$
\mathcal{H}_{D}=D \sum_{i, j} \Delta_{i j}\left(c_{i \uparrow} c_{j \downarrow}+c_{j \downarrow}^{\dagger} c_{i \uparrow}^{\dagger}\right)
$$

where the $\Delta$ matrix describes its symmetry. For local s-wave superconductivity it simply becomes

$$
\Delta_{i j}=\delta_{i j}
$$

but for nodal superconductivity it involves more than one site at a time. In the case of d-wave superconductivity there are two different symmetries, namely $d_{x^{2}-y^{2}}$ symmetry, where

$$
\Delta_{i j}=\left\{\begin{aligned}
1 & , \mathbf{r}_{\mathbf{i}}-\mathbf{r}_{\mathbf{j}}= \pm \mathbf{e}_{\mathbf{x}} \\
-1 & , \mathbf{r}_{\mathbf{i}}-\mathbf{r}_{\mathbf{j}}= \pm \mathbf{e}_{\mathbf{y}}
\end{aligned}\right.
$$

and $d_{x y}$ symmetry with

$$
\Delta_{i j}=\left\{\begin{array}{rl}
1 & , \mathbf{r}_{\mathbf{i}}-\mathbf{r}_{\mathbf{j}}= \pm\left(\mathbf{e}_{\mathbf{x}}+\mathbf{e}_{\mathbf{y}}\right) \\
-1 & , \mathbf{r}_{\mathbf{i}}-\mathbf{r}_{\mathbf{j}}= \pm\left(\mathbf{e}_{\mathbf{x}}-\mathbf{e}_{\mathbf{y}}\right)
\end{array} .\right.
$$

It is also possible to treat non-local s-wave superconductivity with a suitably chosen Weiss field.

As Weiss fields are intruduced to treat broken symmetry phases by using a finite size cluster, the strength of the Weiss field should scale to zero if one increases the cluster size.

\section{Bath Sites}

Besides using one-body terms of the original cluster Hamiltonian and additional Weiss fields in the variation, it is also possible to couple the cluster to additional bath sites. These bath sites are non-interacting and therefore do not change the Luttinger Ward functional - the clustered system stays a valid reference system for the self-energy functional theory. The idea behind the introduction of bath sites is taken from other embedding 
theories such as (cellular) DMFT [KSPB01], where the full lattice problem is mapped to an impurity or cluster that is embedded in a bath of non-interacting electrons.

In VCA, adding additional bath sites to the cluster leads not only an allowed reference system, but also increases the number of degrees of freedom. In this case, at least the hybridization between cluster and bath sites should be included in the set of variational parameters. It turns out that it is necessary to include bath sites in order to be able to detect first order (Mott) metal-insulator transitions in the Hubbard model [BKS $\left.{ }^{+} 09\right]$. Without bath sites or (e.g. magnetic) Weiss fields, the system is either metallic or insulating and can hence only show continuous metal-insulator transitions. This can be seen if one considers a minimal set of variational parameters, namely only the chemical potential of the cluster $\mu^{\prime}$, which is necessary to obtain thermodynamically stable values of the electron density $n$ [AAPH06a]. In the case of the two-dimensional Hubbard model with nearest-neighbor hopping, the filling can be fixed to half-filling by choosing the chemical potential $\mu=U / 2$. If the hopping on the cluster is then isotropically set to $t^{\prime}=t=1$, one finds a maximum of the self-energy functional with respect to the cluster chemical potential. At large $U / t$ the system is gapped and hence an insulator, but this gap shrinks when $U / t$ is reduced. It goes continuously to zero at a critical point $U_{c}$, where the system turns into a metal (see e.g. section 4.1).

The situation is quite different if one adds non-interacting bath sites with chemical potential $\mu_{b}=0$ which are coupled to the correlated bath sites via a hopping term $V$. This hybridization $V$ depicts an additional degree of freedom, which can lead to two stable solutions and in the end allows for the coexistence of metal and insulator.

\section{Evaluation of the Self-Energy Functional}

The central quantity of VCA is the self-energy functional as given by equation (3.2). It consists of three terms which can be further transformed in order to calculate the functional in practice [Pot03a]. In the following, the semi-analytical calculation of the functional is discribed. At the end of this subsection, a brief comparison with and comment on the alternative evaluation of equation (3.2) in a purely numerical way is given. The first term is the grand potential of the reference system, which is given by

$$
\Omega^{\prime}\left[\Sigma^{\prime}\left(t^{\prime}\right)\right]=\Omega^{\prime}\left(t^{\prime}\right)=-T \ln \operatorname{tr}^{\prime} e^{-\frac{\mathcal{H}^{\prime}-\mu N^{\prime}}{T}},
$$

where $N^{\prime}$ denotes the total particle number operator. As the cluster should be chosen such that its eigenenergies can be still computed exactly (using full diagonalization or Lanczos techniques), it is possible to write the trace explicitely by using the eigenenergies of the cluster:

$$
\Omega^{\prime}\left(t^{\prime}\right)=-T \ln \sum_{m} e^{-\frac{E_{m}^{\prime}-\mu N_{m}^{\prime}}{T}}
$$

Once $\mathcal{H}^{\prime}-\mu N^{\prime}$ is fully diagonalized, the grand potential can be calculated for arbitrary temperatures. If the focus is put at low temperatures $T$, the lowest eigenenergies contribute the most and it is possible to calculate only a subset of eigenenergies, for example using a finite-temperature Lanczos algorithm [ADEvdL03]. At zero temperature the grand potential reduces to the ground state energy of the cluster $E_{0}^{\prime}-\mu N_{0}^{\prime}$.

The second term of equation (3.2) is more complicated to calculate. Here, only the main 
steps to arrive at the final expression are outlined. A more detailed calculation which includes some technical aspects is given in Appendix C. The term becomes:

$$
\operatorname{Tr} \ln \left(\frac{-1}{\omega+\mu-\mathbf{t}-\mathbf{\Sigma}(\omega)}\right)=-2 L \sum_{k} \sum_{m} T \ln \left(1+e^{-\omega_{m}(k) / T}\right)+C_{\Sigma} .
$$

The frequencies $\omega_{m}$ are the eigenenergies of the Green function $\left(\mathbf{G}_{\mathbf{0}}{ }^{-1}-\Sigma(\omega)\right)^{-1}$ and $C_{\Sigma}$ is a term that depends on the self-energy and which cancels when the second and third term of equation (3.2) are combined.

Similary, the last term can be rewritten as

$$
\operatorname{Tr} \ln \left(\frac{-1}{\omega+\mu-\mathbf{t}^{\prime}-\mathbf{\Sigma}(\omega)}\right)=-2 L \sum_{m} T \ln \left(1+e^{-\omega_{m}^{\prime} / T}\right)+C_{\Sigma} .
$$

This time, $\omega_{m}^{\prime}$ are the poles of the cluster Green function $\mathbf{G}^{\prime}$. As both the second and the third term contain the cluster self-energy, the term $C_{\Sigma}$ is the same in both terms and cancels when calculating the self-energy functional.

In the end, the self-energy functional can be rewritten as

$$
\begin{aligned}
\Omega\left[\Sigma^{\prime}\right]= & -T \ln \sum_{m} e^{-\left(E_{m}^{\prime}-\mu N_{m}^{\prime}\right) / T}-2 L \sum_{m} T \ln \left(1+e^{-\omega_{m} / T}\right) \\
& +2 L \sum_{n} T \ln \left(1+e^{-\omega_{n}^{\prime} / T}\right) .
\end{aligned}
$$

At zero temperature this equation simplifies drastically to

$$
\Omega\left[\Sigma^{\prime}\right]=F_{0}^{\prime}-2 L \sum_{k} \sum_{\omega_{m}(k)<0} \omega_{m}(k)+2 L \sum_{\omega_{n}^{\prime}<0} \omega_{n}^{\prime}
$$

It is this very formula that will be used throughout the remainder of this thesis to evaluate the self-energy functional.

However, this way of calculating the SEF requires precise knowledge of the poles $\omega_{m}$ and $\omega_{n}^{\prime}$. Within a Band-Lanczos calculation of the cluster Green function this is not much of a problem, but it turns into one when calculating the Green function within a continued fraction representation, see Appendix A. It turns out, that for using the Q-matrix notation of the Green function (see section 3.1.1) a common basis for the excitations of the system is necessary. In contrast to the calculation of $\mathbf{G}^{\prime}$ with the Band-Lanczos algorithm, where multiple starting vectors converge simultaneously, the continued fraction representation of $\mathbf{G}^{\prime}$ via the Lanczos algorithm uses a different Lanczos basis for each component of the cluster Green function. A second problem consists in the form of the continued fraction representation itself - weights and poles of the cluster Green function cannot be obtained easily. One would have to try to extract them from broadened data, which is a hard problem on its own.

The second way of evaluating the SEF consists in calculating equation (3.3) by numerical integration over frequencies $\omega$ and wave numbers $\mathbf{k}$. In this case, the frequency sum is not done analytically and it is sufficient to be able to calculate the Green function at a given frequency $\omega$. This can be done using a continued fraction representation of the cluster Green function and for instance the Lanczos algorithm can be used. In reference [Sén08a], Sénéchal compared both ways of calculating the SEF with respect to accuracy and runtime. As the purely numerical calculation involves only linear-algebraic operations 
on the Green function, it is considerably faster than the semi-analytic calculation scheme once large clusters are involved. However, for small clusters both schemes are comparable with respect to speed of the calculation. In addition, a proper treatment of the numerical frequency integration is essential to obtain precise values for the SEF, whereas the semianalytic calculation treats the frequency summation exactly. Furthermore, the explicit knowledge of the poles within the semi-analytic scheme allows for a study of quantities like the spectral function without any artificial broadening. This can become important for instance when determining phase transitions precisely.

\section{Evaluation of Expectation Values}

After finding a stationary point of the self-energy functional, one is often interested in expectation values of one-body terms at this stationary point. In the following, the evaluation of expectation values by using the Green function at a stationary point is summarized. A more detailed discussion can be found in references [Sén08a, Pot12].

In general, the one-body term can be written as

$$
\mathcal{O}=\sum_{\alpha \beta} o_{\alpha \beta} c_{\alpha}^{\dagger} c_{\beta}
$$

where $o_{\alpha \beta}$ includes site, band and spin indices $\alpha=(i, l, \sigma)\left(\beta=\left(j, m, \sigma^{\prime}\right)\right)$ and has to be chosen accordingly for each one-body observable. As no multi-band models are treated within this thesis, the band index will be skipped from here on. To give a few examples, the electron density $n=\sum_{i, \sigma} c_{i \sigma}^{\dagger} c_{i \sigma}$ would lead to $o_{\alpha \beta}=\delta_{i j} \delta_{l m} \delta_{\sigma \sigma^{\prime}}$. A magnetically long-range ordered state with the magnetic ordering vector $\mathbf{Q}$ can be treated with a proper Weiss field. The strength of the resulting staggered magnetization $m=\delta_{i j} \delta_{l m} \delta_{\sigma \sigma^{\prime}}(-1)^{\sigma} e^{i \mathbf{Q} \cdot \mathbf{r}}$ can be treated as well as the kinetic energy term $E_{\text {kin }}=\sum_{\alpha \beta} \mathbf{t}_{\alpha \beta} c_{\alpha}^{\dagger} c_{\beta}$. When using Nambu's notation or a particle-hole transformation on down spin electrons instead, it is possible to express superconducting Weiss field terms in a similar way and consequently calculate superconducting expectation values.

In the end all these one-body terms have corresponding expectation values which can be calculated as

$$
\begin{aligned}
\langle\mathcal{O}\rangle & =\frac{1}{N} o_{\alpha \beta}\left\langle c_{\alpha}^{\dagger} c_{\beta}\right\rangle \\
& =\frac{1}{N} \operatorname{Tr}(\mathbf{o G})=\frac{T}{N} \sum_{m} e^{i \omega_{m} 0^{+}} \operatorname{tr}(\mathbf{o G}), \quad i \omega_{m}=\pi(2 n+1) .
\end{aligned}
$$

The expectation value $\left\langle c_{\alpha}^{\dagger} c_{\beta}\right\rangle$ can be calculated by using a contour integration of the Green function:

$$
o_{\alpha \beta}\left\langle c_{\alpha}^{\dagger} c_{\beta}\right\rangle=T \oint_{\mathcal{C}} \frac{\mathrm{d} z}{2 \pi i} \operatorname{tr}(\mathbf{o G}) f^{\mathrm{FD}}(z)
$$

Analogously to the calculation in the appendix, one inserts two unitary matrices $\mathbf{U U}^{\dagger}=\mathbf{1}$ which diagonalize $(\mathbf{o G})^{-1}$ and obtains its poles $\xi_{m}$ and weights $a_{m}$. Due to the residue theorem one then transforms the contour integration into a sum over the residues of the integrand which coincide with the poles of $\mathbf{G}$. In addition, one changes into the previously mentioned mixed representation of cluster and superlattice indices and does a Fourier transformation with respect to the superlattice. By doing this, the matrices $\mathbf{o}(\tilde{\mathbf{k}})$ and $\mathbf{G}(\tilde{\mathbf{k}}, z)$ are still matrices with respect to the cluster sites, but are now functions of 
superlattice momentum $\tilde{\mathbf{k}}$. In the end, the integration thus amounts to a summation of the product of the Fermi-Dirac distribution and the residues $R_{m}(\tilde{\mathbf{k}})$ that are connected with the poles $\xi_{m}(\tilde{\mathbf{k}})$ over these poles:

$$
\langle\mathcal{O}\rangle=\frac{T}{N} \sum_{\tilde{\mathbf{k}}} \sum_{m} R_{m}(\tilde{\mathbf{k}}) f^{\mathrm{FD}}\left(\xi_{m}(\tilde{\mathbf{k}})\right) .
$$

At zero temperature the contour used previously reduces to a contour that surrounds the poles of the Green function on the negative real axis counterclockwise. This is due to the fact that only excitations from the ground state to states with higher energy are allowed and hence energy differences and thereby excitation energies which correspond to poles of $\mathbf{G}$ have to be negative. Another way to see this is to consider the zero temperature limit of the Fermi-Dirac distribution:

$$
\lim _{T \rightarrow 0}\left(e^{z / T}+1\right)^{-1}=\left\{\begin{array}{ll}
0 & , \operatorname{Re} z>0 \\
1 & , \operatorname{Re} z<0
\end{array} .\right.
$$

Similar to the calculation of the self-energy functional at zero temperature, the calculation of one-body expectation values simplifies to

$$
\langle\mathcal{O}\rangle=\frac{1}{N} \sum_{\tilde{\mathbf{k}}} \sum_{\omega_{m}(\tilde{\mathbf{k}})<0} R_{m}(\tilde{\mathbf{k}}) .
$$

In the case of an $L$-site cluster with two spin species $N=2 L$ and the residues $R_{m}$ can be expressed by the spectral weights of the Green function. Using the $\mathbf{Q}_{\mu n}$ matrices of section 3.1.1 the residues can be rewritten as

$$
R_{m}(\tilde{\mathbf{k}})=\sum_{\alpha \beta}\left(\mathbf{o}_{\alpha \beta}(\tilde{\mathbf{k}}) \overline{\mathbf{Q}}_{\alpha m}(\tilde{\mathbf{k}}) \overline{\mathbf{Q}}_{\beta m}^{*}(\tilde{\mathbf{k}})\right)=\operatorname{tr}\left(\overline{\mathbf{Q}} \mathbf{o}^{*}\right)(\tilde{\mathbf{k}}) .
$$

As long as the ,strength" of the one-body term is part of the set of variational parameters, the corresponding expectation value fulfills thermodynamic stability. This means that evaluating the expectation value as a trace over the Green function or as a functional derivative of the grand potential leads to the same result [AAPH06a]:

$$
\frac{1}{N} \operatorname{Tr}(\mathbf{o G})=\langle\mathcal{O}\rangle=\frac{\partial \Omega}{\partial o}
$$

\section{Details of the Numerical Approach}

In this subsection several numerical details for the implementation of VCA are discussed that have been used to obtain the results shown in the following chapters. In the program that I wrote to do the calculations, whose results are used and discussed in the following chapters, a few libraries were used. Those for numerical integration and numerical optimization are discussed below. In addition to that, the Eigen matrix library $\left[\mathrm{GJ}^{+} 10\right]$ in combination with the intel Math Kernel Library (MKL) has been used for efficient and parallelized matrix and vector operations. However, for matrix diagonalizations the $L A$ $P A C K$ routines which are also included in MKL were used instead of the diagonalization routines of Eigen. 
Legendre Transformation of the Grand Potential. For most of the calculations that follow in the next chapters the electron density $n$ of a model system should stay fixed to a certain value $n_{f}$. One possibilty of realizing this density would be to calculate the stationary point of the SEF for different values of the chemical potential $\mu$ and determine the value where the corresponding electron density $n(\mu)=n_{f}$. An easier method consists in doing a Legendre transformation of the grand potential to the free energy $F=\Omega+\mu N$, where $N$ denotes the particle number inside the cluster, as shown in reference [BP10]. In addition to the stationarity condition for the SEF $\partial \Omega / \partial \xi^{\prime}=0$ the Legendre-transformed functional also has to fulfill the condition $\partial \Omega / \partial \mu=0$ in order to have the correct particle number $\left\langle\sum_{i} n_{i}\right\rangle=N$. In practice, it turns out that the SEF usually has a maximum with respect to both the chemical potential of the cluster $\mu^{\prime}$, and the chemical potential $\mu$.

Treatment of the Superlattice Moment k. After tiling the system into a superlattice of identical clusters, one does a Fourier transformation on the superlattice coordinate $\tilde{\mathbf{r}}$, which is then denoted by $\tilde{\mathbf{k}}$. For an infinite system one would arrive at a continuum of $\tilde{\mathbf{k}}$-values and after reperiodizing the cluster Green function any point in the Brillouin zone can be realized, see figure 3.1 in subsection 3.1.3.

An approximation which is often used consists in considering a finite number of clusters that make up the superlattice. For example taking $N=(101)^{2} 2 \times 2$ clusters that form a two-dimensional square lattice would lead to a discretized Brillouin zone with an equidistant grid of $N$ points. It can be further improved by doing an interpolative k summation instead, as proposed by Balzer and Potthoff [BP10]. This approximation is justified for most situations if one is interested in calculating quantities like spectral functions, but it can also be used to calculate the self-energy functional or expectation values at the stationary point of the SEF. However, in some cases it is not sufficient to include ,only" 10,000 points inside the Brillouin zone in order to reach a certain precision of the self-energy functional. The position of the stationary point would then still change considerably when increasing the number of $k$-points. Therefore, it would be necessary to check in every situation which number of $k$-points is necessary in order to achieve a satisfactory precision for the calculation of the SEF.

For example, when studying the Mott metal-insulator transition in the next chapter the curvature of the SEF changes when crossing the transition line - the SEF becomes very flat with respect to some variational parameters like the chemical potential of the cluster. This has first of all consequences for the convergence criteria that are applied for finding stationary points in the SEF landscape and it also implies that it can be difficult to find a number of $k$-points that is sufficient for calculating the SEF with reasonable precision throughout a parameter scan. Determining this number of $k$-points by hand for ,characteristic" parameter sets of the calculation is on the other hand not desirable.

An easier approach consists in directly demanding a certain precision of the value of the SEF after the summation over $k$-values of the Brillouin zone. Therefore, the summation over discrete $k$-points is replaced by the numerical integration over $\mathbf{k}$ in the Brillouin zone. In practice the numerical integration is done in my program by using the so-called CUHRE-routine of the CUBA library which was developed by Hahn [Hah05, Hah06]. This routine goes back to the (DC)UHRE algorithm by Berntsen, Espelid and Genz [BEG91b, BEG91a] which uses cubature rules to refine the integration grid by globally adaptive subdivision. Iteratively the region with the largest estimated error is bisected 
and the two new subregions are added to the set of regions which are considered for the next iteration. Once the estimated error falls below a given threshold value or a maximal iteration number is exceeded, the algorithm stops. Its advantage over most other integration routines lies in its precise estimation of the error and its efficiency in low dimensions. Furthermore, the integration routine is already parallelized which leads to a significant speed up.

Usually it proved to be sufficient to require a relative error of less than $10^{-6}$ for the calculation of the SEF. The number of $k$-points then turns out to be normally between 2000 and 150,000 points. In some cases the relative error had to be lowered to $10^{-8}$, but a maximal iteration number of 200,000 $k$-points was rarely exceeded.

In this way, the precision of the calculation of the SEF can be controlled well. The following subsection shows how the stationary point of the SEF landscape is determined.

Determination of Stationary Points of the Self-Energy Functional. Within the variational principle of self-energy functional theory the one-body parameters that enter the set of variational parameters have to be varied in order to identify stationary points of the self-energy functional. In practice it turns out that stable stationary points are in general saddle points with respect to the variational parameters, i.e., a maximum with respect to a subset and and a minimum with respect to the rest of the parameters. The search for stationary points can be done in different ways. Besides the choice of a specific (usually local, iterative) optimization algorithm, one has to decide whether to use a multi-dimensional or nested one-dimensional optimization schemes.

After testing different optimization algorithms, the optimization for low-dimensional variational spaces was finally done by using the „constrained optimization by linear approximations"by Powell described in [Pow94] in the implementation of NLopt used [Joh]. Using the Nelder-Mead simplex method described in [NM65] and the principal axis method of Brent [Bre73] from the same library also lead to good results. However, these algorithms for local optimization are gradient-free and only work well and efficiently if the starting point is chosen close to the stationary point. After a stationary point is found one usually does parameter scans, which explains why gradient-free optimization algorithms work so well. If the new parameter set does not differ too much from the previous one, it can be expected that the position of the stationary point also changes little. Hence taking the values of the previous stationary point are already a good starting point for the new configuration.

Using optimization algorithms which include the gradient such as (quasi-)Newton algorithms, especially the BFGS algorithm [BGLS03], proved to be more efficient when the position of the stationary point was not known. Especially combining quasi-Newton algorithms with a principal axis transformation as proposed by Balzer and Potthoff [BP10] should be more efficient in general. However, as an approximation of the gradient of the SEF has to be calculated numerically which requires the calculation of the SEF at additional points, these algorithms are mostly useful for high-dimensional sets of variational parameters. Especially when separating the variational parameters $\xi_{\max }^{\prime}$ which lead to a maximum of the SEF and those $\xi_{\text {min }}^{\prime}$ which lead to a minimum of the SEF the dimension of the subsets is usually not large enough to render quasi-Newton algorithms efficient. Usually the SEF shows a maximum with respect to the chemical potential of the cluster $\mu^{\prime}$ and, if the functional is Legendre transformed, also with respect to the chemical potential $\mu$. With respect to other variational parameters it is often a minimum.

One possibility of judging the "quality" of the determination of the stationary point is 
to check for thermodynamical stability of expectation values that are connected with the variational parameters, i.e. to check whether using the self-energy functional and the Green function to evaluate the expectation value leads to the same result. In this way it is also possible to find reasonable values for the convergence criteria of the optimization. For most of the cases studied in the following chapters, it was sufficient to demand an absolute precision of $10^{-5}$ for the chemical potential and an absolute precision of $10^{-3}$ for other variational parameters to reach thermodynamical stability. The higher precision in the determination of the chemical potential has to do with the flat SEF with respect to $\mu^{\prime}$ and $\mu$.

In order to achieve stability when using high-dimensional variational spaces, it furthermore proved to be important to do a nested optimization: In each step of the minimization of the SEF with respect to $\xi_{\text {min }}^{\prime}$ the SEF was first optimized with respect to $\mu^{\prime}$ (and $\mu$ ). Especially when treating superconductivity this was necessary to stabilize the electron density $n$ within the minimization procedure.

Calculation of Fermi Surfaces. Once the stationary point of the SEF has been found, the approximate lattice Green function allows for the calculation of the spectral function as discussed in section 3.1.3. For stationary points that amount to a metallic solution of the system, it is possible to obtain the Fermi surface from the spectral function. Within the definition used here, the Fermi energy corresponds to $\omega=0$. In a two-dimensional Brillouin zone the Fermi surface then amounts to the intersection of the spectral function with the $\omega=0$ plane, that is

$$
\mathbf{k} \in \mathrm{BZ}, \quad \text { s.t. } \quad A(\mathbf{k}, \omega=0) \neq 0 .
$$

Two aspects are important to determine the Fermi surface numerically. When using the Q-matrix notation for the spectral function, the poles and weights are given explicitly without any broadening. In practice the condition that the weight has to be non-zero is hard to fulfill exactly as numerical errors might lead to small but finite artifacts. To circumvent this problem the Fermi surface is either plotted color-coded or a threshold value $\epsilon$ is introduced and the condition weakened to $A(\mathbf{k}, \omega=0)>\epsilon$, with $\epsilon \sim 10^{-3}$. The other aspect relates to the finite resolution when choosing a discretization in $k$, e.g. with an equidistant grid. It is then nearly impossible to fulfill the condition that a pole of the spectral function coincides with the Fermi energy. A possible way out consists in introducing a small energy window with width $\tilde{\epsilon}$ around the Fermi energy and to demand that $A(\mathbf{k},|\omega|<\tilde{\epsilon})=\epsilon$. This in turn poses the problem that low-lying electron-/holeexcitations are included. A too large $\tilde{\epsilon}$ therefore results in the wrong shape of the "Fermi surface". To exclude this, a careful analysis of the emerging Fermi surface has to be done. This is done in the following chapters by checking that reducing the width of the energy window and increasing the number of $k$-points in the grid does not change the Fermi surface qualitatively. For most cases a width of $\tilde{\epsilon}=10^{-1}$ was sufficient for an equidistant grid of 40, $000 k$-points. 
Treating Local Spin Interactions: Application of the

\section{Variational Cluster Approximation to the Kondo Lattice}

\section{\begin{tabular}{l|l} 
Model & 3.2 .3
\end{tabular}}

In section 3.1.4, it was already shown that perturbative cluster approaches can be used to describe pure spin systems, but the investigation of phases with broken symmetry is not included in such a treatment. Although a derivation of a spin self-energy functional and a spin-VCA based on this functional is in principle possible (see e.g. [FP14]), the appearance of complex excitations leads to a breakdown of the theory at small temperatures [FP14].

The approach to magnetism and superconductivity that will be pursued here is to use the variational cluster approximation for the Kondo lattice model. Therefore, first of all one has to revisit the foundations of self-energy functional theory to check whether VCA can be applied to this model.

Self-energy functional theory is based on the Legendre-transformed Luttinger-Ward functional. The Luttinger-Ward functional is defined for electronic Green functions, i.e. correlators of the type $\left\langle\left\langle c_{\alpha}^{\dagger} c_{\beta}\right\rangle\right\rangle$. Operators that act on the local spins of the Kondo lattice model are therefore not covered. In contrast to the Hubbard model, not only creation and annihilation of electrons, but also spin-flip operations of the f-spins should be considered for the Kondo lattice model.
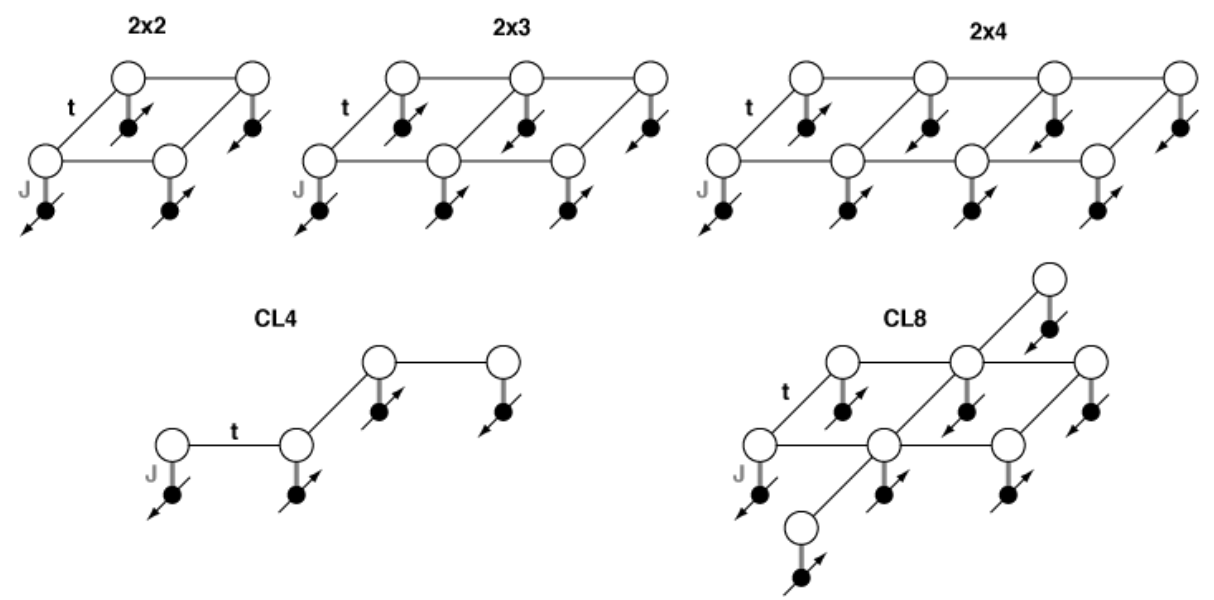

Figure 3.10: The cluster geometries that will be considered for investigating the Kondo lattice model within VCA. As the lower clusters have sites with minimal coordination number, they are somewhat pathological. In contrast, the upper clusters can be grouped together: They are all $L \times x$ clusters and it is this set of clusters for which most of the results will be shown.

A pragmatic approach used in this thesis is to use standard variational cluster approximation in terms of the Green function and the self-energy of the electronic degrees of freedom only. Yet the "full" Kondo lattice Hamiltonian is included for the calculation of quantities on the cluster. This means that the Green function still includes only correlators for conduction electrons and no correlators between the f-spins or between electrons and spins. 
When using the Lehman representation of the Green function, the eigenenergies $E_{m}$ of the Hamiltonian as well as the eigenstates $|m\rangle$ enter the calculation, see section 3.1.1. They are now calculated with the Hamiltonian of the Kondo lattice model. In order to determine these eigenenergies a basis has to be used which also includes all possible configurations of the f-spins on the cluster. The local basis for states of this Hamiltonian has therefore size eight: On each site there are up to two electrons and (exactly) one spin with two different configurations.

One way of thinking about this approach would be to consider the spin degrees of freedom to be integrated out such that only the electronic degrees of freedom remain in thermodynamic quantities, which can be derived from the VCA Green function and/or the approximated grand potential. Still, the interactions with the f-spins are included in the cluster self-energy. It is only the spin-flip excitations that do not enter explicitly via some spin self-energy. As the coupling to f-spins is local, the tiling of the two-dimensional lattice into a superlattice of identical clusters still only "cuts" hopping terms between these clusters. The coupling to the spins is the same in the original and the reference system.

However, as the Kondo coupling term between conduction electrons and f-spins is not of the form of electron-electron interactions which were used in the derivation of the Luttinger-Ward functional, the validity of the approximation would have to be checked with a thorough derivation of a similar functional which includes the coupling term.

Although no Coulomb interaction is present in the model, the self-energy functional theory cannot be automatically considered to be exact. This can be seen when considering the Kondo lattice model as the limiting case of the periodic Anderson model.

The periodic Anderson model is made up of two electron bands, a conduction band with 'c'-electrons and a second band which models the f-shell and is therefore called 'f'-band. Electrons can move inside and between the bands via suitably chosen hopping parameters. A local Coulomb interaction term acts on the f-band only, whereas the electrons on the 'c'-band move freely. When sending the Coulomb repulsion to infinity and demanding half-filling on the f-band, the Kondo lattice model can be derived as an effective model via a Schrieffer-Wolff transformation [SW66, SN02].

As the periodic Anderson model includes interacting electrons for which the LuttingerWard functional is well-defined, it could be assumed that the self-energy functional theory is still valid in the limit $U \rightarrow \infty$. Although the interaction which enters the LuttingerWard functional acts on the f-band which after mapping corresponds to the spins of the KLM, it also leads to the coupling term between these spins and conduction electrons. Therefore part of the interaction is still present in the KLM albeit not connecting two electronic propagators that take part in the Green function.

At this point it has to be noted, that within this thesis the Kondo lattice model and not the periodic Anderson model is treated within the approximation described in the previous paragraphs.

This motivates the choice of the variational cluster approximation for the electronic degrees of freedom of the model. In reverse, this means that only electronic degrees of freedom can be described in a thermodynamically stable way. Other quantities like the (staggered) magnetization of the f-spins can only be calculated on the cluster as the spin operators do not enter the Green function. Still, the local correlator between electrons and the f-spin on site $i$ can be obtained as a derivative of the energy with respect to the 
coupling strength, $\left\langle\mathbf{s}_{i}^{c} \mathbf{S}_{i}^{f}\right\rangle=\partial E_{0} / \partial J$.

For the electronic degrees of freedom standard VCA applies, including additional Weiss fields that allow for the study of symmetry broken phases. Due to the increased Hilbert space dimension only small clusters are treatable. Most of the clusters that will be used for analyzing the Kondo lattice model are shown in figure 3.10. Especially the clusters without „dangling“ sites such as the $2 \times 2$ and $3 \times 2$ cluster will be considered.

\section{\begin{tabular}{l|l} 
Survey of Other Numerical Approaches & 3.3
\end{tabular}}

Different numerical approaches have been used to study the two-dimensional Hubbard and Kondo lattice model. Some of them are briefly discussed in the following. The results of these techniques will be used afterwards as a reference for results obtained within this thesis using variational cluster approaches.

The first subsection focuses on techniques that were used to particularly study the universality class of the Mott transition in two-dimensional Hubbard models. In the second subsection a survey of numerical approaches to the two-dimensional KLM sets the stage for the investigations on the KLM in chapter 5 .

\section{The Mott Transition in Two-Dimensional Hubbard Models}

In order to understand the ground-phase diagram of the organic charge-transfer salts and the Mott transition, a lot of numerical studies investigated the half-filled Hubbard model on an anisotropic triangular or a frustrated square lattice. This includes meanfield and Hartree Fock treatment [MYI06], U(1) gauge theory [LL05], fluctuation exchange (FLEX) approximation [KK98, KM99], variational Monte Carlo (VMC) studies [LST05, WYTI06], dynamical mean-field theory (DMFT) [KLR00], cellular DMFT [KT06, SWGK11, ST12, SHT12, $\mathrm{ARC}^{+}$16], VCA and Dual Fermions [LTP+15], dynamical cluster approximation [PBK04, $\left.\mathrm{DXC}^{+} 15\right]$, exact diagonalization [KMF07] and path integral renormalization group [KI01, MWI02].

Due to deficiencies of the techniques when it comes to critical exponents, there are only very few numerical studies on lattice systems which make a statement on the universality class of the Mott transition. One of the first of those studies is the work by Assaad and Imada [AI96], who used determinantal Quantum Monte Carlo (QMC) to investigate the Green function $G(\mathbf{r}, \omega)$ of large lattices (up to $16 \times 16$ ) directly at zero temperature. They extracted the localization length $\xi_{l}$ from calculating the Green function at long distances within the Hubbard gap, where $G(\mathbf{r}, \mu) \propto e^{-|\mathbf{r}| / \xi_{l}}$. For the localization length they then found critical behavior $\xi_{l} \propto\left|\mu-\mu_{c}\right|^{-\nu}$ with $\nu=0.26 \pm 0.05$, which agrees with the expectation from hyperscaling, namely $\nu=1 / 4$ and a dynamical exponent of $z=4$.

In a subsequent QMC study together with Furukawa [FAI96] they accompanied their results of the insulating side by calculations of the compressibility $\kappa=\partial \delta / \partial \mu$ in the metallic phase, where $\delta$ stands for the doping and $\mu$ denotes the chemical potential. In the metallic phase close to half-filling, the doping $\delta=1-n$ is given as the derivative of 
the singular part of the free energy $f_{s}$ with respect to the chemical potential $\mu$. By assuming hyperscaling, they were then able to connect the doping to the shift of the chemical potential $\Delta=\mu-\mu_{c}$ via $\delta \propto|\Delta|^{\nu d}$. As the compressibility allows to determine this shift via $\kappa \propto|\Delta|^{-1 / 2}$, they could determine the correlation length exponent in another way by approaching the critical point from the metallic side and obtained $\nu=0.21 \pm 0.04$.

Misawa and Imada investigated quantum criticality around the metal-insulator transition within a frustrated extended Hubbard model on a square lattice with Hartree-Fock approximation [MI07]. In their paper they studied the metal-insulator transition in symmetrybroken phases, which are in the case of the extended Hubbard model a charge ordered phase, when the nearest neighbor interaction $V$ dominates over local Coulomb interaction $U$, and a antiferromagnetic ordered phase for $U>4 V$. Within the mean-field approximation transitions from both insulating phases into the metallic phase should have the same nature. Considering a free-energy expansion and numerically solving Hartree-Fock self-consistency equations they then investigated critical exponents when approaching the marginal quantum critical point along several trajectories. Both approaches led to the same sets of critical exponents, which differed on the insulating and metallic side.

At high temperature, their calculations gave classical Ising exponents, but at zero temperature the transition was found to be continuous. These two regions are separated by a marginal quantum critical point which leads to different critical exponents. These exponents on the metallic side, especially $(\beta, \gamma, \delta)=(1,1,2)$, match those of the "unconventional" criticality that was found experimentally in the group of Kanoda [KMK05, KMK09, $\left.\mathrm{FMT}^{+} 15\right]$. At small temperatures they observed a crossover from Ising to unconventional critical exponents.

In the end the authors argue, that their assumption of a preexisting gap does not have to be caused by symmetry breaking such as charge or antiferromagnetic ordering, but could also be the correlation-induced gap of a Mott insulator at low dimensions (without long-range order), which could not have been described within their mean-field treatment. As a consequence they expect the critical exponents also to be found when investigating the Mott transition beyond mean-field theory.

Using single-site dynamical mean-field theory for the Hubbard model, Kotliar et al. [KLR00] assumed the existance of a finite temperature transition and derived a Landau functional to extract the critical exponent $\delta=3$ analytically. This result is not surprising, as the critical exponent for Ising universality at infinite dimensions coincides with the one of mean-field criticality, where it is known that $\delta=3$ [Gol92].

Nevertheless, there exist two papers which study spin and charge criticality for the $2 \mathrm{D}$ Hubbard model using plaquette C-DMFT [SWGK11, ST12], a real-space cluster generalization of DMFT, but albeit using the same technique on the same model, they come to different conclusions. In the work by Sentef et al. the extracted exponents close to the second-order end point of the Mott transition seem to fit Ising universality [SWGK11]. Sémon and Tremblay investigated the importance of subleading corrections for the Mott critical point using C-DMFT and found that fitting with a single exponent over a broad region away from the critical point leads to a critical exponent of $\delta \approx 2$. When taking into account subleading corrections to the Mott transition, $\delta=3$ gave much better agreement with the data both for DMFT and C-DMFT [ST12]. However, as these techniques are mean-field techniques, its value for the discussion of the universality class of the Mott 
transition is questionable.

In a detailed study, Sato et al. used cellular dynamical mean-field theory and included vertex corrections inside the cluster [SHT12]. When investigating thermodynamic criticality by looking at the double occupancy $d$ at both the insulating and the metallic side of the transition they found mean-field behavior near the critical end point, namely $\left(1 / \delta^{-}, 1 / \delta^{+}\right)=(0.32 \pm 0.05,0.30 \pm 0.04)$. However, when studying electronic proporties via the optical conductivity they tried to fit the Drude weight with a scaling function as $\left|D-D_{c}\right| \propto\left|U-U_{c}\right|^{1 / \delta_{c}^{ \pm}}$. Using the jackknife method for an error analysis to estimate the critical exponent with the optical weight led to $\left(1 / \delta^{-}, 1 / \delta^{+}\right)=(0.15 \pm 0.04,0.16 \pm 0.03)$, which is unconventional in the sense that it does not match mean-field or Ising exponents. Even within the scaling theory of Papanikolaou et al. $\left[\mathrm{PFF}^{+} 08\right]$, where the conductivity scales with the energy density of the Ising universality class could not reproduce the mean-field exponent that was extracted from the double occupancy data.

To conclude this survey, different numerical approaches have been applied to the twodimensional Hubbard model. Even within the same technical framework, different results are obtained for the universality class of the Mott transition, see table 3.1. Besides differences in the techniques and their application, the discrepancy of these studies are also due to the different approaches to extracting meaningful exponents. Based on the assumptions that enter the scaling function, different quantities that allow for scaling are used.

\begin{tabular}{|l|l|l|}
\hline Technique/ Theory & Critical exponents & Ref. \\
\hline \hline QMC & $\nu=0.26 \pm 0.05$ & {$[$ AI96] } \\
QMC & $\nu=0.21 \pm 0.04$ & {$[$ FAI96] } \\
\hline Hartree Fock & $(\beta, \gamma, \delta)=(1,1,2)$ to $(\beta, \gamma, \delta=)(1 / 2,1,3)$ & {$[$ MI07] } \\
CDMFT & $\delta=1.64 \pm 0.13,1.72 \pm 0.17,2.08 \pm 0.10$ & {$[$ SWGK11] } \\
\hline DMFT & $\delta=3$ & {$[$ KLR00] } \\
CDMFT & $\delta=3$ & {$[$ ST12] } \\
CDMFT & $1 / \delta=0.15 \pm 0.04,1 / \delta=0.32 \pm 0.05$ & {$[$ SHT12] } \\
\hline
\end{tabular}

Table 3.1: Summary of the critical exponents of the theoretical studies mentioned in the text. The two studies in the middle support unconventional criticality $((\beta, \gamma, \delta)=(1,1,2))$, the last three find mean-field universality $(\beta, \gamma, \delta)=(1 / 2,1,3)$.

However, none of these numerical studies investigated the anisotropic two-dimensional Hubbard model with frustration with respect to the Mott transition. The route that is pursued in chapter 4 to account for quantum criticality is different as the interchain hopping $t_{\perp}$ is used as a control parameter. Nevertheless, the results will be compared to some of the just mentioned numerical studies when possible, e.g. at the isotropic point of the model $\left(t_{\perp}=t\right)$. 


\section{\begin{tabular}{l|l} 
The Kondo Lattice Model & 3.3 .2
\end{tabular}}

The Kondo lattice model has the already discussed limits of infinitely large coupling, where local singlets between conduction electrons and f-spins form, and of weak interaction, where effective RKKY interactions determine the physics. In between, the Doniach diagram describes the competition of both processes and gives a rough picture, which nevertheless is only based on comparing the energy scales of both limiting cases.

Due to the lack of (exact) analytical tools to tackle the full Kondo lattice problem, one has to resort to approximate numerical techniques to piece together a consistent picture of the region of intermediate coupling strengths. Nevertheless, before numerical tools became powerful enough to approach the Kondo lattice model, analytical or semi-analytical approaches lead to valuable insights. Here, some of the results that have been obtained with numerical techniques and which will prove important for comparisons in the subsequent subsections are summarized. What follows is not a complete list but rather a selection of studies which highlights the most important and reliable results out of a large set of techniques.

One of the most important ideas that also inspired a lot of numerical techniques is the large-N expansion [And81]. Motivated by the large magnetic spin degeneracy of $\mathrm{f}$ moments $N=2 j+1$ in heavy fermion systems Anderson proposed to use $1 / N$ expansions, which was later used to construct various approximations, such as slave boson mean-field theories. For the Anderson lattice (PAM) in the $U \rightarrow \infty$ limit Lavagna et al. used such a Kondo-boson $1 / N$ expansion and found a d-wave superconducting instability [LML87]. This superconductivity was shown to emerge due to slave-boson exchange and not to be associated with spin fluctuations.

One of the first approximations which has been applied to solve the Kondo lattice problem is mean-field theory. Already in 1979 Lacroix and Cyrot found that for a three-dimensional Kondo lattice at zero temperature three different phases emerge as a function of Kondo coupling $J$ and electron filling $n$ [LC79]. Their phase diagram shows an antiferromagnetic phase at small coupling strength down to a critical electron filling $n_{c}$ below which the system orders ferromagnetically. This phase extends at small fillings even to quite high coupling strengths. From a certain coupling strength on, the ground state of the system turns out to be a Kondo singlet state.

In a thorough variational Monte Carlo study by Asadzadeh et al. the two-dimensional Kondo lattice model was for comparison also revisited by means of mean-field theory [ABF13]. There, the antiferromagnetic region for electron fillings $n \gtrsim 0.8$ was found to be split into a region with small Fermi surface (' $\mathrm{AF}_{s}$ ') for small to intermediate coupling strengths and a region with hole-like Fermi surface (' $\mathrm{AF}_{h}$ ') close to the transition to the paramagnet. The existence of the $\mathrm{AF}_{s}$ phase is in agreement with previous mean-field approaches, see e.g. references [ZY00, SVS04], where regions were identified in which the antiferromagnetic metal also has a small Fermi surface and Kondo screening is absent. A drawback of all these mean-field studies is the overestimation of antiferromagnetic order (the critical value $J_{c}$ differs by a factor of roughly two) [CA01] and at half-filling it is problematic to fulfill the condition of having exactly one f-spin per site locally and not only in average [WO07]. 
Already in 2007 Watanabe and Ogata investigated the KLM via variational Monte Carlo (VMC) and studied the Kondo screening which shows in different Fermi surfaces of the antiferromagnetic metal [WO07]. Within VMC one or several trial wave functions are used in combination with a variational principle. The technique can be applied at zero temperature, but in the end the results depend on the variational wave function that is used. They are therefore usually biased towards one of the phases that is realized in the system. In the VMC phase diagram, the paramagnetic and ' $\mathrm{AF}_{h}$ ' phases are also found and the qualitative picture is even similar to the mean-field one. A big difference to meanfield theory is the existence of an antiferromagnetic metal with a small electronic Fermi surface ('AF ${ }_{e}^{\prime}$ ), which is in parts made up of the f-spins. That means that f-spins and conduction electrons hybridize and in contrast to $\mathrm{AF}_{s}$ Kondo screening is still present. However, the absolute values differ roughly by a factor of two and close to half-filling even the curvature of the transition lines is different [ABF13]. Within VMC the transition between $\mathrm{AF}_{h}$ and the paramagnetic metal turn out to be of second order, but the transition from $\mathrm{AF}_{h}$ to $\mathrm{AF}_{e}$ and below $n \approx 0.8$ from paramagnetic metal directly to $\mathrm{AF}_{e}$ were of first order. At half-filling this continuous transition was first found by Wang et al. in 1994 [WLL94]. In the VMC study of Asadzadeh et al. d-wave superconductivity was found in the paramagnetic, but not inside the antiferromagnetic phase [ABF13]. Later, the same authors showed that superconductivity could enter in the transition region between paraand antiferromagnet if magnetic frustration is introduced in the system [AFB14].

A technique which is conceptually similar to VCA, so-called dynamical cluster approximation (DCA) [HMJK00, MJPH05], was used by Martin et al. to investigate the evolution of the Fermi surface when crossing the antiferromagnetic transition line [MA08, MBA10]. DCA can be considered as a cluster extension of DMFT. It is a cluster technique that works directly in momentum space and coarse grains the Brillouin zone. Although DCA still shows finite-size effects, the use of quantum Monte Carlo solvers at finite temperature allows to use quite large clusters. The authors of [MA08, MBA10] found evidence for three distinct regions in the phase diagram and showed that the Fermi surface topologies of the two antiferromagnetic metal phases are continuously connected and correspond to those that can be obtained by using a spin-density wave approximation. The transition from para- to antiferromagnet is found to be continuous, and at all finite coupling strengths no Kondo breakdown was observed. Later, Bercx and Assaad could show that instead of a Kondo breakdown scenario, the Fermi surface topologies change according to a Lifshitz transition [BA12].

Recently, Peters and Kawakami used a real-space formulation of dynamical mean-field theory to investigate the evolution of the Fermi surface again [PK15]. They found metallic spin-density wave (SDW) phases away from half-filling. Inside this SDW phase the Fermi surface (FS) changed from small to large when increasing the coupling strength, similar to the Fermi surface evolution in the antiferromagnetic case discussed before. However, only the transition from the SDW phase with large FS to the paramagnetic metal is of second order. At small electron filling the transition from the SDW phase with small FS to the paramagnet is first order and a quantum critical point is avoided.

The density matrix renormalization group (DMRG) technique [Whi92], which is one of the most powerful numerical tools for the study of one-dimensional systems and which has been used in recent years also to investigate two-dimensional systems [YHW11, DMS12, 
SW12] has also been applied to Kondo ladders. In case of N-leg Kondo ladders at halffilling Xavier calculated spin and charge gaps and found evidence for the presence of a quantum critical point [Xav03]. However, close to half-filling the investigation of the binding energy of two holes does not support superconductivity. In a follow-up study of the KLM with additional antiferromagnetic coupling between localized spins Xavier and Dagotto instead found indications for the existence of d-wave superconductivity close to half-filling, which is mediated by antiferromagnetic fluctuations [XD08]. Unfortunately, a careful two-dimensional finite size scaling in two dimensions was beyond their capabilities as energies did not converge well. The precise determination of $J_{c}$ was also not possible [XD08].

In 2013, Bodensiek et al. used dynamical mean-field theory (DMFT) with a numerical renormalization group (NRG) solver and unexpectedly found robust s-wave superconductivity, which is connected to the formation of heavy fermion bands and strong local spin fluctuations $\left[\mathrm{BŽV}^{+} 13\right]$. The anomalous expectation value of this superconducting phase was found to be maximal at a coupling strength of $J / t \approx 2.4$ and a filling of $n \approx 0.9$. For large $J / t$ the authors motivated the pairing mechanism perturbatively and supported their numerical study by a spin-fluctuation theory based on Eliashberg theory, which after certain uncontrolled approximations allows for s-wave superconductivity [BŽ $\left.{ }^{+} 13\right]$. Although in the mean-time the DMFT $+\mathrm{NRG}$ results could be verified with an independent program [Pet14], no other numerical technique has found similar local s-wave superconductivity since.

Furthermore, it can be shown that in contrast to the case of d-wave superconductivity, the equations of motion of the non-retarded gap equation for local s-wave superconductivity is zero [Gez16]. In the scenario put forward in reference $\left[\mathrm{BŽV}^{+} 13\right]$ to give a possible explanation for the formation of s-wave superconductivity retardation effects are indeed essential. Still, one might want to ask whether both retarded and non-retarded s-wave superconductivity should not coincide in the thermodynamic limit.

The result which is closest to the unconventional local s-wave superconductivity is the s-wave superconductivity that was found by Masuda and Yamamoto in 2015 [MY15]. In their study they used VCA for the periodic Anderson model (PAM) and found s-wave superconductivity between c- and f-electrons. However, as they studied the PAM at small interaction strengths only and as their s-wave superconductor seems to be conceptionally different, it is far from clear that it will survive in this form in the limit $U \rightarrow \infty$. Besides analyzing the difference of the s-wave superconductor of the PAM and the potential s-wave superconductor of the KLM, checking whether other kinds of superconductivity superpose the s-wave superconductor in case of the PAM remains an open question.

In 1995 Shi et al. used high order series expansions in $t / J$ (up to 8th order) and found evidence for a continuous phase transition from Kondo insulator to antiferromagnetic state around $J_{c} \sim 1.43$ for a square lattice [SSGW95]. At half-filling, numerically exact and therefore most reliable results for the Kondo lattice model have been obtained by Assaad in 1999 with an auxiliary-field quantum Monte Carlo method (QMC) [Ass99]. This work at zero temperature was then extended to finite temperatures by Capponi and Assaad using the same technique [CA01]. One of the main findings was the precise determination of the critical coupling strength $J_{c} / t=1.45 \pm 0.05$ at which the continuous transition from an antiferromagnetic to spin-gapped insulator takes place. The finite-size extrapolated 
values of the quasiparticle gap are also considered to be exact and used as a reference later in this chapter.

Considering the investigation of superconductivity in the Kondo lattice model one of the most significant studies is the one by Otsuki [Ots15] who applied the dual-fermion approach, which can be viewed as a diagrammatic extension of DMFT [RKL08, RKLG09]. Within this approach at finite temperatures, the critical value of the quantum critical point between the antiferromagnetic and the paramagnetic phase could be improved a lot with respect to DMFT. The extrapolation to zero temperature leads to a value of $J_{c} / t=$ $1.35 \pm 0.05$ which agrees within error bars with the QMC result of Assaad. By measuring the Fermi surface area down to the lowest accessible temperatures $(T \lesssim 0.01)$, it was furthermore possible to identify small and large Fermi surfaces as well as a crossover region between them. The key point of this study is the comparison of the leading eigenvalues for all possible superconducting pairing types. Otsuki finds d-wave superconductivity at weak coupling and low temperatures, but at intermediate coupling strengths the ground state is a p-wave spin-singlet superconductor. He concludes that in this region superconductivity emerges from the incomplete quasiparticles of the crossover region between small to large Fermi surface, which undergo critical antiferromagnetic fluctuations. 



\section{Mott Quantum Criticality in Two Dimensions}

In this chapter, the anisotropic frustrated two-dimensional Hubbard model (see eq. 2.1) introduced in section 2.1 is investigated. The focus is set on the Mott metal-insulator transition (MIT) and a different route is proposed to account for its low critical end point $T_{c}$. Considering the fact that quantum fluctuations are enhanced in low-dimensional systems with spatial anisotropy, the interchain hopping $t_{\perp}$ is used as a tuning parameter. The effect of this anisotropic hopping amplitude is investigated and the location of the putative quantum critical point at $T=0$ is estimated in the phase diagram.

Throughout this chapter, the next-nearest-neighbor hopping $t^{\prime}$ is always chosen proportional to the interchain hopping $t_{\perp}$ in order to maintain a certain relative frustration when tuning $t_{\perp}$. Both the paramagnetic phase, where the transition happens between a metal and a Mott-Hubbard insulator, and the antiferromagnetic phase, where the metal goes over to a Mott-Heisenberg insulator, are discussed.

The outline of this chapter is as follows. In the first two sections $(4.1,4.2 .5)$, the paramagnetic phase of the model is investigated and the next-nearest-neighbor hopping is fixed to $t^{\prime}=-0.25 t_{\perp}$.

The first section contains a preliminary study using standard variational cluster approximation to study the Mott transition. In this treatment, the reference system consists of a cluster without Weiss fields or bath sites and phase diagrams for different cluster sizes are calculated. Finally, the paramagnetic phase is studied within VCA using a $2 \times 2$ plaquette with one bath site per correlated site in section 4.2. The metal-insulator transition is found to be discontinuous for large $t_{\perp}$, but for small interchain hopping it becomes continuous. In both regions, the insulating and metallic phases are investigated and the zero-temperature VCA data is compared to finite-temperature cellular dynamical meanfield theory (CDMFT) results of M. Raczkowski. A consistent picture emerging from these two techniques is presented and the connection of the results to measurements on layered organic conductors is discussed. The extraction of critical exponents from VCA data is shown for the sake of completeness in section 4.2 .5 and its validity is commented on.

In section 4.3, the antiferromagnetic phase of the model is investigated for two different relative frustration strengths $t^{\prime} / t_{\perp}$. There, the insulator is of Mott-Heisenberg type and the position of the metal-insulator transition line is determined. Differences between the phase diagram of the paramagnetic and antiferromagnetic case are discussed. Finally, the findings are analyzed in context of putative marginal quantum criticality and further studies are motivated.

Parts of this chapter, especially of section 4.2, were published as „Mott Quantum Criticality in the Anisotropic 2D Hubbard Model", Physical Review Letters 116, 086403 (2016) in collaboration with Salvatore R. Manmana, Thomas Pruschke, Fakher F. Assaad and Marcin Raczkowski. Section 4.2.5 was motivated by one of the referee reports for this paper in which the referee explicitly asked for critical exponents at the MIT. The figures in this publication were prepared by me, nevertheless figures in this thesis that are 
taken unchanged from the publication are indicated as such. Since section 4.2 is based on the publication, parts of it might resemble its manuscript. All calculations using variational cluster approximation were done by me using my own program code. The CDMFT calculations at finite temperature were done by Marcin Raczkowski, who also initiated the project. The manuscript was mainly written by me and Marcin Raczkowski, but all authors were included in the scientific discussion and contributed to the manuscript.

\section{First Steps Towards the Mott Transition}

Before including bath sites in the reference cluster, in this section the anisotropic frustrated two-dimensional Hubbard model is investigated with different clusters that do not include uncorrelated bath sites. The focus is set on the metal-insulator transition in the paramagnetic phase. By choosing different clusters, the dependence of the phase diagram on the cluster size can be analyzed. The clusters used in this section all have a rectangular geometry and are shown in the left panel of figure 4.1.

However, before studying a metal-insulator transition, the criterion that allows to discriminate both phases from each other is revisited.

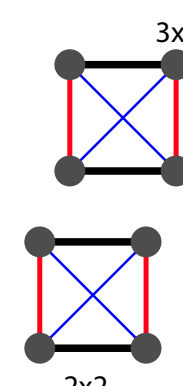

$2 \times 2$
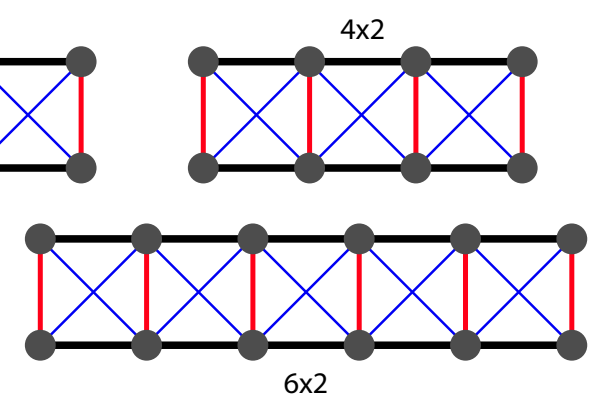

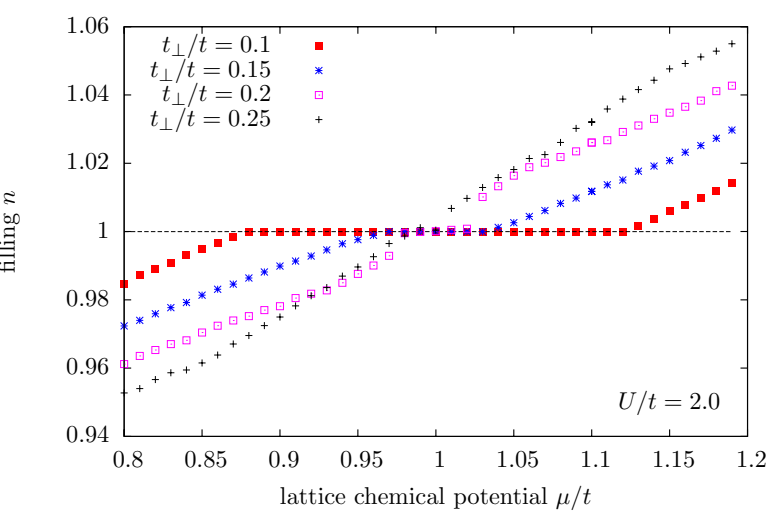

Figure 4.1: Left panel: Different clusters without additional bath sites: $2 \times 2$ plaquette, $2 \times 4$ cluster, $4 \times 2$ cluster, $6 \times 2$ cluster. Right panel: The electron density $n$ as a function of chemical potential $\mu$ at $U / t=2.0$ for a $4 \times 2$ cluster. Curves are shown for different values of interchain coupling $t_{\perp} / t$ close to the metal-insulator transition. For small $t_{\perp}$, there exists a distinct plateau and the system is insulating, for large interchain hopping it is metallic.

As already mentioned in section 2.1, one of the quantities that are often used to distinguish metal from insulator is the compressibility $\kappa=-\partial n / \partial \mu$, where $n$ denotes the electron filling and $\mu$ is the chemical potential. In case of an insulator, the compressibility is zero; for a metal it is finite. When plotting the electron density as a function of chemical potential, the insulator shows accordingly a plateau at half-filling $(n=1)$, whereas the metal possesses a finite slope.

Another possibility consists in investigating the Drude weight. An investigation of the spectral function and the density of states $(\operatorname{DoS}(\omega))$ is possible within VCA and has been described in section 3.1.3. Checking whether the system has a gapped density of states around $\omega=0$ at half-filling or whether $\operatorname{DoS}(\omega=0) \neq 0$ without a gap is therefore a valid alternative.

Here, the compressibility is used as a criterion, but it was checked that investigating the 
density of states leads to the same results.

In case of the isotropic unfrustrated two-dimensional Hubbard model, choosing $\mu=U / 2$ leads to half-filling. Here, the model contains different nearest- and next-nearest-neighbor hopping terms and, as a consequence, the chemical potential $\mu$, which corresponds to halffilling, has to be determined. When scanning a parameter range for the correct chemical potential, the $n$-vs.- $\mu$ curve is obtained as a side-product and can be used to determine the compressibility.

The smallest set of variational parameters that are necessary to obtain a thermodynamically stable solution within VCA consists in taking the chemical potential of the cluster $\mu^{\prime}$ only [AAPH06a]. In the following, only $\mu^{\prime}$ is used as a variational parameter and the electron density $n$ is determined as a function of the chemical potential $\mu$. The right panel of figure 4.1 shows the electron density as a function of $\mu$ at half-filling for a $4 \times 2$ cluster at $U / t=2.0$ for different interchain hopping strengths $t_{\perp} / t$. For small values of $t_{\perp} / t$, a pronounced plateau is visible, which shrinks in size when increasing the interchain hopping. In this region, the compressibility is zero and the system is hence insulating. Finally, at a certain value $t_{\perp}^{c} / t$ the plateau is gone and the system is metallic.

Using the compressibility as a criterion, one can investigate the model as a function of Coulomb repulsion $U / t$ and interchain hopping $t_{\perp}$. For the isotropic two-dimensional model, it is expected that the system is metallic for small interaction strength. At some critical value $U_{c} / t$, the system should then turn into a Mott-Hubbard insulator. In the other limit of decoupled chains $\left(t_{\perp} / t=0\right)$, the system is gapped for any finite interaction strength $U / t$, and only at $U=0$ metallic. As the model allows to continuously change the parameter $t_{\perp} / t$, it can be expected that the value of $U_{c} / t$ smoothly becomes smaller when reducing $t_{\perp}$.

Figure 4.2 shows the phase diagrams for this model obtained by using $4 \times 2$ and $6 \times 2$ clusters.
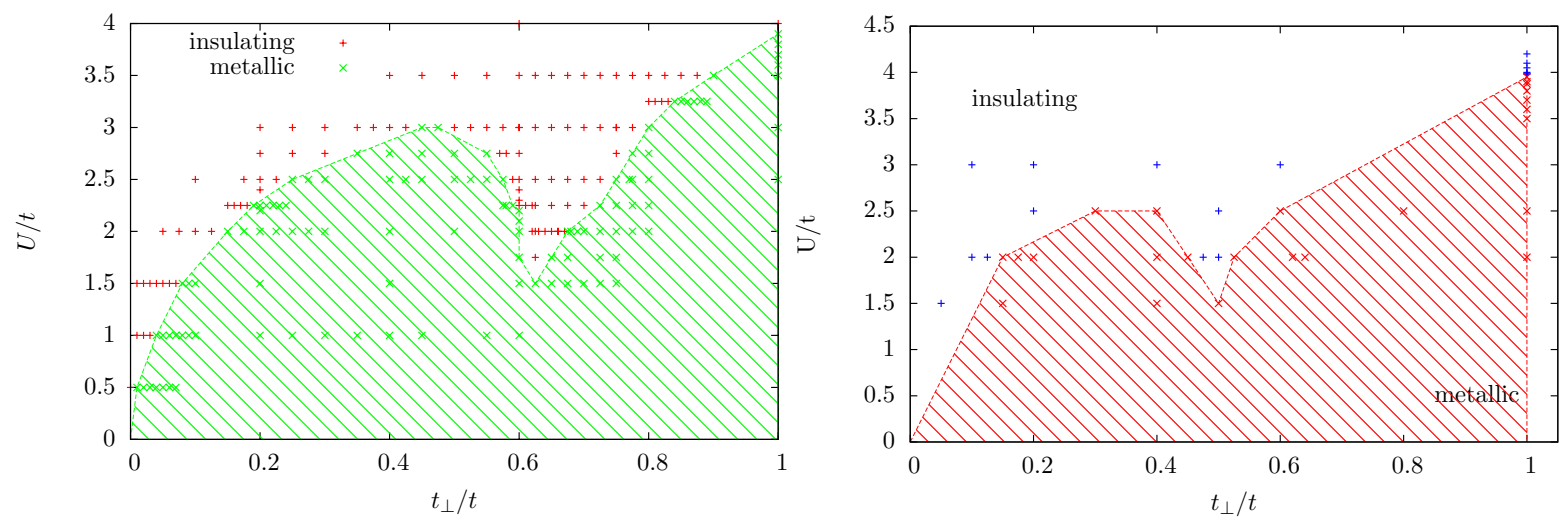

Figure 4.2: Phase diagram for a $4 \times 2$ cluster (left panel) and a $6 \times 2$ cluster (right panel). Remarkably, there is a dip at intermediate interchain coupling strengths, which in the second case is close to $t_{\perp} / t=0.5$. Lines are a guide to the eye only.

Focussing on the results using the $4 \times 2$ cluster first, the transition at small interchain hopping strengths $0<t_{\perp} / t \lesssim 0.4$ shows the expected behavior. The critical interaction strength $U_{c} / t$ increases smoothly when raising $t_{\perp}$. Close to the transition, it turned out 
to be difficult to distinguish a plateau from a linear segment with small but finite slope. In the region of large hopping strengths $0.8 \lesssim t_{\perp} / t \leq 1$, the results also match the expectations. $U_{c} / t$ decreases as the interchain hopping is reduced. The metal shows a large slope in the $n$-vs.- $\mu$ curve around $n=1$ and is quite easily distinguished from the insulator.
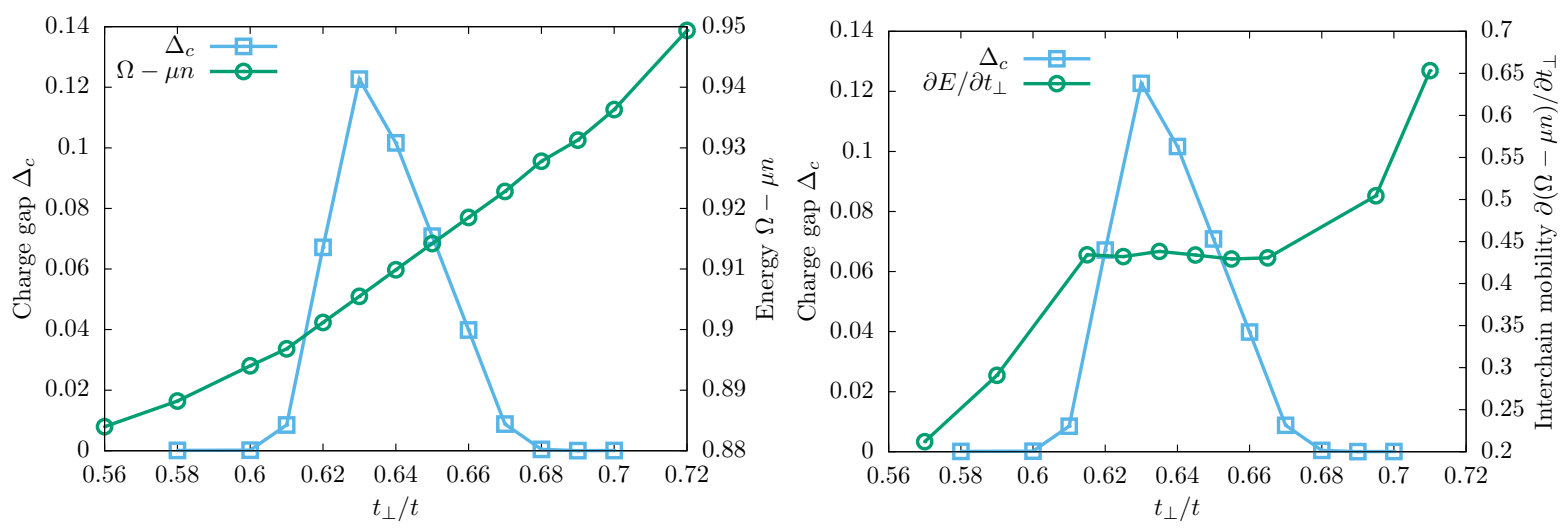

Figure 4.3: Left panel: The charge gap $\Delta_{c}$ and the energy $\Omega-\mu n$ as a function of interchain hopping $t_{\perp} / t$ in the regime of the unexpected insulating region at fixed $U / t=2$. Right panel: Interchain mobility $\partial(\Omega-\mu n) / \partial t_{\perp}$ in the same region; the charge gap is shown again for comparison. Data for both panels were obtained with a $4 \times 2$ cluster.

However, in the region of intermediate hopping strength $0.4 \lesssim t_{\perp} / t \lesssim 0.8$ the phase diagram shows a dip at $t_{\perp}^{d} / t \approx 0.62$. This unexpected extend of the insulating region down to an interaction strength of $U / t=1.75$ is robust and can also be observed in other quantities than only the compressibility. Figure 4.3 shows the energy and the electron mobility in interchain direction $\partial E / \partial t_{\perp}$ for a sweep of $t_{\perp}$ through the dip region at constant interaction strength $U / t=2$. The size of the plateau in the $n$-vs.- $\mu$ curve is identified as the charge gap $\Delta_{c}$ and is shown in the figure for comparison. In all three quantities, the metal-insulator transitions can be identified. The plateau size is finite in the insulating region from $t_{\perp}^{-} \approx 0.61$ to $t_{\perp}^{+} \approx 0.67$. Within this region, the energy increases linearly with the interchain hopping, as can be seen in the constant interchain mobility $\left\langle t_{\perp}\right\rangle=\partial E / \partial t_{\perp}$. This is in contrast to the metallic phases that connect to this phase both for larger and smaller interchain hopping.

In order to clarify whether the metallic phases for small and large interchain hopping differ, their Fermi surface is investigated. Figure 4.4 shows characteristic Fermi surfaces for both metallic regions at $U / t=2$. The value, for which the Fermi surface is shown in the right panel of figure 4.4, is deep in the metallic phase. There, most of the weight of the spectral function at $\omega=0$ closely follows the non-interacting dispersion. For large interchain hopping strengths, the Fermi surface resembles the large Fermi surface of an ordinary metal.

However, as a finite number $N_{c}$ of 12 -site clusters is used to calculate the spectral function, an artifact of the approximate reperiodization of the cluster Green function (see section 3.1.3) is clearly visible in the plot. Due to the 12 sites inside the cluster, the Brillouin zone is made up of 12 patches with $N_{c} \mathbf{K}$-vectors. Hence, only when increasing the number of clusters, the proximate region around the centers of these 12 patches (corresponding to $\mathbf{K}=0$ ) can be investigated. As a consequence, the Fermi surface on the right side of 
figure 4.4 is split into several pieces. Nevertheless, the similarity to the non-interacting dispersion and the big differences to the Fermi surface in the left panel are striking. For small values of $t_{\perp} / t$, the Fermi surface close to the transition shows closed patches, so-called electron and hole pockets. In reference [ET05], Essler and Tsvelik describe weakly coupled one-dimensional Hubbard chains and also find these kind of pockets. They argue that adding interactions to the warped one-dimensional Fermi surface leads to the formation of hole and electron Fermi pockets of a higher-dimensional compensated metal [ET05]. In a previous analysis of the same model that is studied here, these pocket structures were found at finite temperatures and interpreted as being remnant 1D signatures of Umklapp scattering [RA12]. There, the region of intermediate hopping strengths was not investigated and the pockets were less pronounced. Simple tight-binding calculations also show that dispersions with structures similar to the pockets rather stem from one-dimensional than from two-dimensional order D.

Finding these pocket structures within VCA at zero temperature up to quite large hopping strengths of $t_{\perp} / t \approx 0.5$ is quite remarkable. When adopting the interpretation of being remnant 1D features, this would imply that one-dimensional fluctuations are still the crucial factor at intermediate hopping strengths. The pocket structure of the metal at small interchain coupling will be investigated more thoroughly in section 4.2.
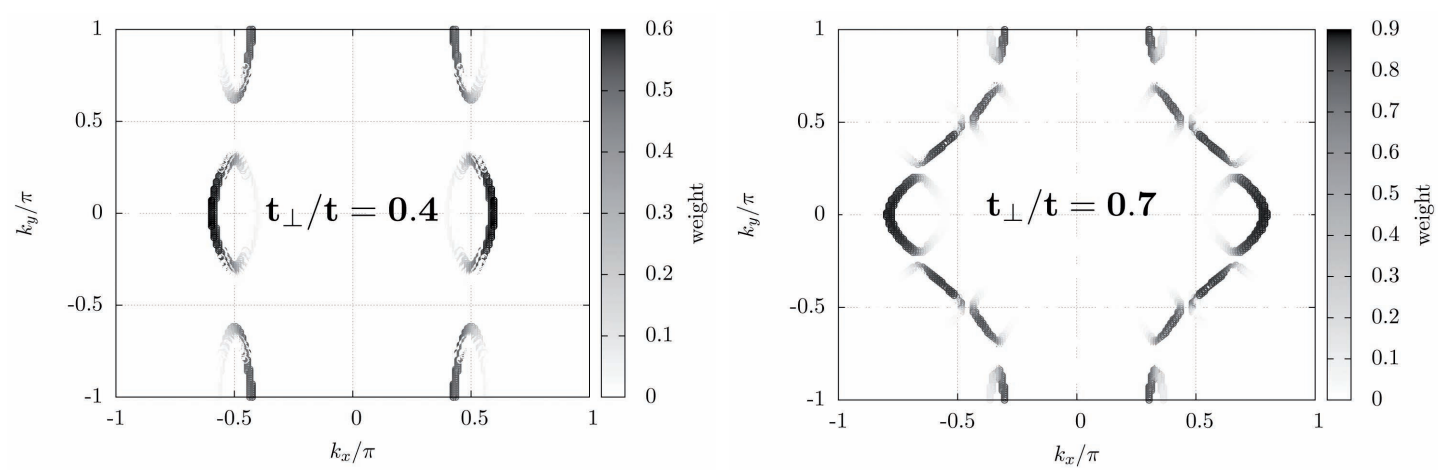

Figure 4.4: Fermi surfaces at $U / t=2$ to the left side of the dip $\left(t_{\perp} / t=0.4\right.$, left figure) and to the right side of the dip $\left(t_{\perp} / t=0.7\right)$ using a $6 \times 2$ cluster.

It was shown that the metals at $U / t \approx 2$ for $t_{\perp}<t_{\perp}^{-}$and $t_{\perp}>t_{\perp}^{+}$differ in their Fermi structure. However, at small interactions such as $U / t=1$ they are not separated anymore by an insulating region. A smooth change between the Fermi surfaces has to be expected and might explain the dip in the transition line. It has to be noted that the non-interacting Fermi surface changes qualitatively at $t_{\perp} / t=2 / 3$ (compare with figure 2.1). A Van Hove singularity appears at the point where the warping of the Fermi surface, due to $t_{\perp}$, leads to an intersection of the dispersion with the Brillouin-zone boundary at $k_{x}= \pm \pi$. For larger interchain hopping, the Fermi surface extends over the full range of $\mathbf{k}_{x}$ and $\mathbf{k}_{y}$ values - it changes its character from quasi-1D to quasi-2D. Such a Lifshitzlike transition at finite interaction strength could be an explanation for the dip in the phase diagram. As the pocket structure of the Fermi surface at small interchain coupling only shows close to the transition, i.e. for large interaction strength, and also follows the non-interacting dispersion closely for small interaction strength, a precise determination of such a transition at intermediate interaction strength is difficult. 
When linking the existence of electron and hole pockets of the Fermi surface to the presence of remnant one-dimensional scattering, the large value of $t_{\perp}^{d}$ is surprising. However, most of the discussion was based on a $4 \times 2$ cluster only, but finite-size effects have to be considered. Figure 4.2 shows phase diagrams for the two largest clusters with ladder geometry that were investigated. Compared to the $4 \times 2$ cluster, the shape of the transition line for the $6 \times 2$ cluster is qualitatively the same, but the position of the dip in the transition line is shifted to $t_{\perp}^{d} / t \approx 0.5$.

Also, the size of the dip seems to decrease when increasing the cluster size, but a true finite-size extrapolation would necessitate larger clusters. A combination of VCA with another cluster solver than Band Lanczos might allow for such an extrapolation in future studies. In reference [RA12], Raczkowski and Assaad found less pronounced pockets at finite temperature via cluster dynamical mean-field theory where they could use a quantum Monte Carlo solver. They found the pockets using a $8 \times 2$ and a $4 \times 4$ cluster.

As the metallic region with Fermi-surface pockets is quite broad for the largest cluster size used here, it is plausible that the region even exists for much larger clusters and is not an artifact of small cluster size or the ladder geometry of the cluster.

To summarize the results shown in this subsection, the phase diagram of the model has been determined in the paramagnetic phase; figure 4.2 shows examples for the two largest clusters used. The system is metallic for small $U / t$ and turns into a Mott-Hubbard insulator at a critical interaction strength $U_{c}\left(t_{\perp}\right)$, which defines the transition line. For small interchain hopping strength the metal was shown to have a Fermi surface with electron and hole pockets. At large $t_{\perp}$ the Fermi surface does not show these pockets, but forms the common Fermi surface of a two-dimensional metal instead.

However, when investigating the order of the metal-insulator transition, it turns out that it is continuous for all interchain hopping strengths $t_{\perp}$. Unfortunately, this is not necessarily rooted in the physical model, but can rather be an artifact of the method.

When using VCA without bath sites, the system turns out to be either metallic or insulating. An adequate way for the determination of the transition point is to calculate the compressibility. As this amounts to probing for a plateau in electron density $n$ as a function of chemical potential $\mu$, and as $n(\mu)$ should be unique around half-filling, it becomes clear that anything but a continuous vanishing of the plateau is not possible. This changes when non-interacting bath sites and an hybridization between correlated sites and bath sites are added to the cluster. In the following, this route is pursued to investigate the order of the Mott transition. Results obtained for the simplest quantum cluster technique, which contains the coupling to a bath site, so-called dynamical impurity approximation (DIA), are shown in the appendix E. Remarkably, it is shown in E that the finite-temperature picture of a first-order transition with a pronounced coexistence region breaks down when zero temperature is approached. However, as DIA does not include spatial fluctuations, a different variational cluster approach is used for further studies. Section 4.2 addresses the problem with a cluster that includes bath sites and is sufficiently large to allow for Umklapp scattering and antiferromagnetic fluctuations. 

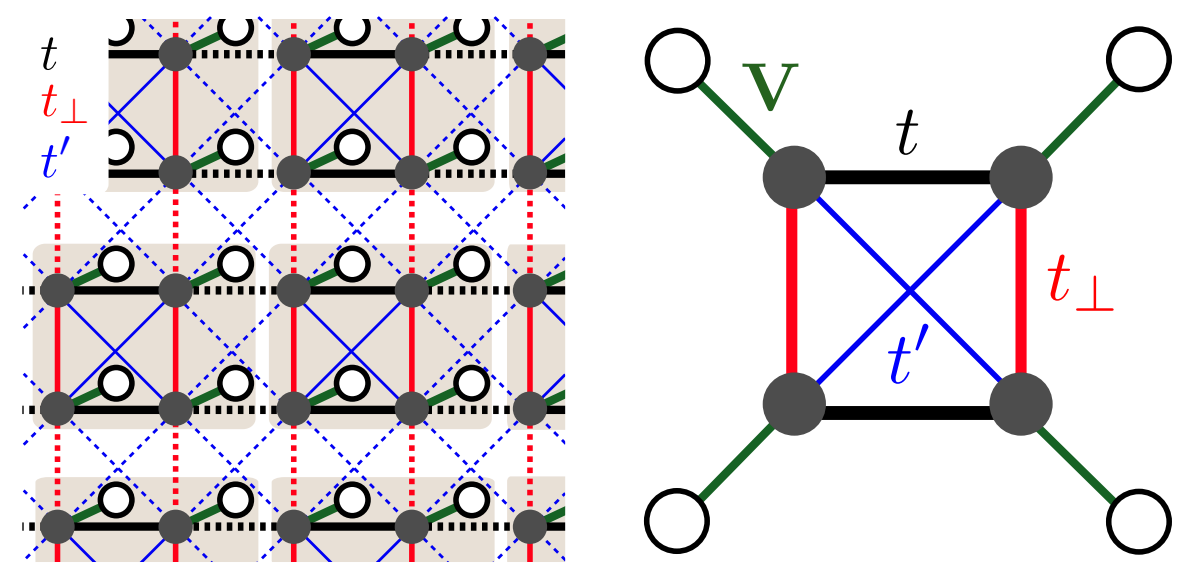

Figure 4.5: Cluster tiling of the lattice system with the reference cluster used in this section. In the $2 \times 2$ plaquette cluster, each correlated site is additionally coupled to a non-interacting bath site (open circles) with hybridization strength $V$.

\section{Mott Transition in the Paramagnetic Phase $\mid 4.2$}

In this section, the results of studying the paramagnetic phase are presented right at the beginning in subsection 4.2.1. Starting from the phase diagram, the different quantities that have been analyzed are discussed in subsection 4.2.2 and it is explained how they lead to the transition line presented in 4.2.1. These include the ground state energy, the double occupancy, and the $V$-dependence of the self-energy functional. In subsection 4.2.4, the Fermi surface of the metallic phase is analyzed and discussed in the context of the order of the Mott transition. Afterwards, the results are compared with finite-temperature CDMFT results. Section 4.2.5 shows how insights into the critical exponents of the Mott transition can be gained in principle and how far VCA can contribute to the discussion of its universality class.

Throughout this section, the Mott transition of the model is investigated in its paramagnetic phase using a $2 \times 2$ cluster with one additional bath site per correlated site, see figure 4.5. This cluster allows one to capture both the 1D Umklapp scattering process opening a gap in the half-filled band $\left[\mathrm{CCK}^{+} 04\right]$ and short-range 2D AFM spin fluctuations, and has to be considered as being the minimal unit cell that is suitable. It has been used already within the variational cluster approximation to investigate the isotropic unfrustrated Hubbard model on the square lattice $\left[\mathrm{BKS}^{+} 09\right]$. In this study the Mott transition was found to be of first order. As the uncorrelated bath sites have to be included when setting up the Hilbert space and diagonalizing the cluster Hamiltonian, it is an effective eight-site cluster and also has an corresponding computational cost. Still, its moderate size allows to scan a broad parameter space. This is even more important when including an antiferromagnetic Weiss field in the next section, as the minimal necessary set of variational parameters contains four parameters there.

Here, the hybridization $V$ between correlated and bath sites, as well as chemical potentials of the reference system $\mu^{\prime}$ and the lattice system $\mu$, respectively, are chosen as variational parameters. The influence of additionally varying the hopping terms on the cluster was checked for different interchain hopping strengths. It did not lead to qualitative differ- 
ences of the results shown in the following compared to a variation of $\mu, \mu^{\prime}$ and $V$ only. Therefore, the hopping terms were chosen to be equal to their lattice analogues and not treated as additional variational parameters.

The VCA results at zero temperature are in the following compared to finite-temperature results obtained by $M$. Raczkowski $\left[\mathrm{LMP}^{+} 16\right]$ with CDMFT [KSPB01]. Both techniques can be described within the framework of self-energy functional theory and can be viewed to be complementary. In CDMFT, a cluster of $N_{c}$ interacting impurities is dynamically coupled to an effective bath. Using a quantum Monte Carlo (QMC) solver, the impurity problem can be solved and the coupling to the bath is determined self-consistently. The drawback of using a QMC solver for this model is the fermionic sign problem, which renders large clusters and low temperatures not accessible. With the $2 \times 2$ plaquette cluster and a Hirsch-Fye solver temperatures down to $T=t / 40$ could be reached.

\section{\begin{tabular}{l|l} 
The Phase Diagram & 4.2 .1
\end{tabular}}

The main results of this section are summarized in the ground-state phase diagram in figure 4.6. It shows the estimate of the critical interaction strength $U_{c}$, at which the system undergoes a transition between Mott-insulating and metallic phases, the transition line, in the full range of $t_{\perp} / t$ between the 1D and 2D regimes. From reference [BHP08] it is known that VCA yields the Mott phase for any finite interaction strength $U>0$ in the 1D limit. This is in agreement with the exact Bethe-ansatz solution [LW68] and the bosonization approach [Gia97].

As shown in figure 4.6, this changes dramatically upon coupling the chains via $t_{\perp} / t>0$. The single-particle hopping $t_{\perp}$ shifts the critical interaction $U_{c}$ towards a finite value and enables the interaction-driven MIT. Initially, $U_{c}$ increases steeply with $t_{\perp}$, but then grows nearly linearly, as it is expected for the MIT controlled by the ratio of Coulomb interaction to kinetic-energy gain. For $t_{\perp} / t>t_{\perp}^{c} / t \approx 0.2$, the MIT line is found to be of first order, consistent with former studies on the frustrated 2D Hubbard model [PBK04, KT06, WYTI06, OMTK08, YKK09, LIM09, DXC ${ }^{+}$15]. In contrast, in the strongly anisotropic case with $t_{\perp} / t \leq 0.2$, it marks a smooth metal-insulator crossover even at zero temperature. The systematic reduction of the critical end point $T_{c}$ identified within CDMFT supports this scenario (see the inset of Fig. 4.6). All of these aspects consistently suggest that $t_{\perp}$ is a control parameter that tunes the nature of the Mott transition from strong first order in the $2 \mathrm{D}$ limit to continuous at $t_{\perp}^{c} / t \simeq 0.2$.

The phase diagram is complemented by insets that show the Fermi-surface topology for values of the interaction close to the transition $U \lesssim U_{c}$. It changes when tuning $t_{\perp} / t$, and three different regions can be identified. For small to intermediate $t_{\perp}$ the $1 \mathrm{D}$ FS is warped and due to the presence of interactions, electron and hole Fermi pockets of a higherdimensional compensated metal are formed [ET05]. In the region $0<t_{\perp} / t / t<t_{\perp}^{c} / t$, the area of the surface pockets vanishes smoothly when approaching the transition line, while for values $t_{\perp}^{c} / t<t_{\perp} / t \lesssim 0.7$, the area jumps abruptly to zero when tuning the interaction strength over the critical value $U_{c} / t$. In the third region $\left(t_{\perp} / t \gtrsim 0.7\right)$, the compensated metal structure of the FS disappears and goes over to a conventional large FS, which coincides with the topological Lifshitz transition of the noninteracting FS. 


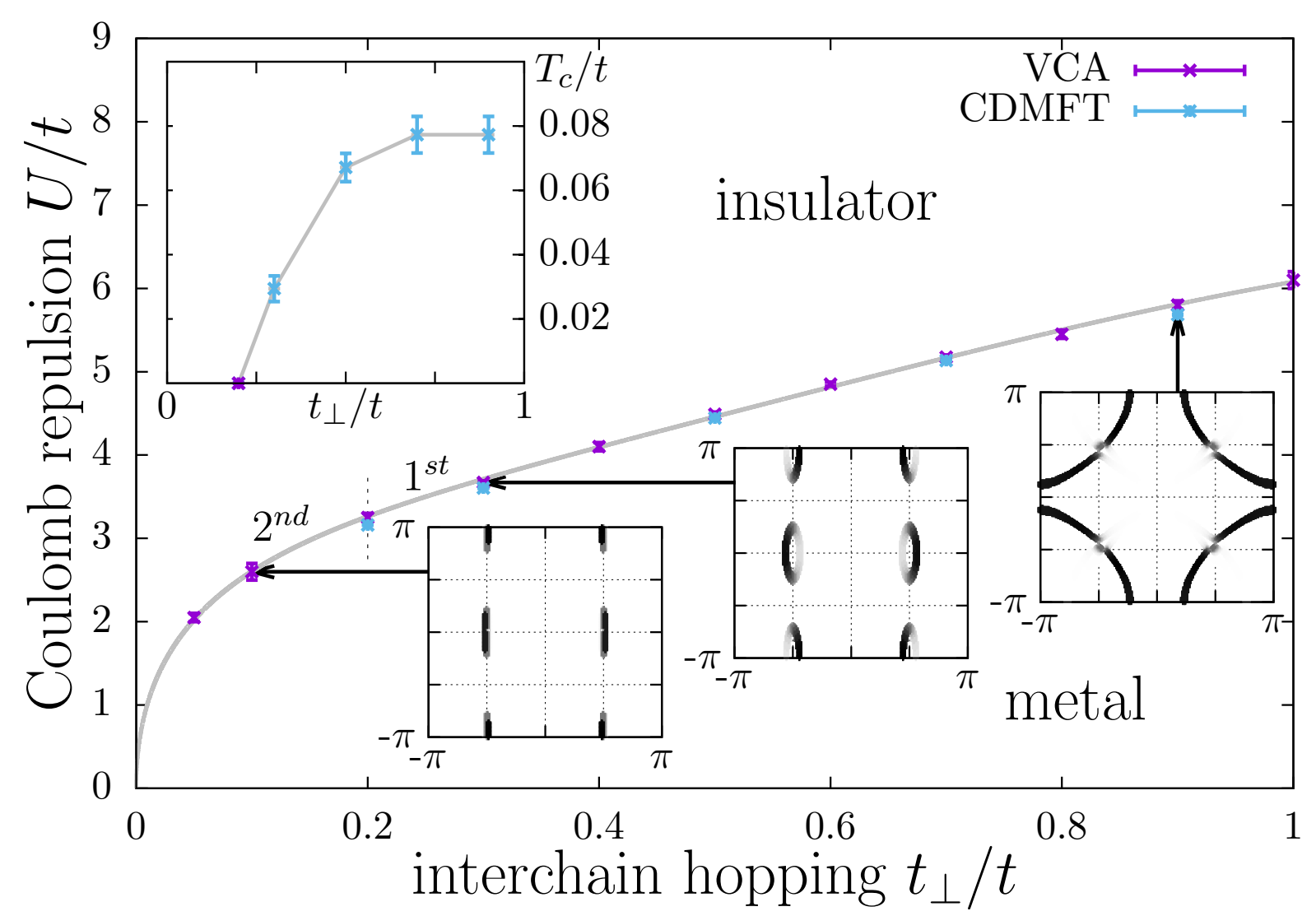

Figure 4.6: Metal-insulator phase diagram of the half-filled Hubbard model as obtained by the zero-temperature VCA and CDMFT at $T=t / 40$. (Top inset) The combined VCA and CDMFT estimate for the critical temperature $T_{c}$ terminating the first-order MIT; $T_{c}$ is driven down to zero at $t_{\perp}^{c} / t \simeq 0.2$, thus providing evidence for the quantum critical nature of the MIT. (Bottom insets) FS topology close to the critical interaction $U_{c}$ in different regions of the phase diagram indicated by arrows. CDMFT data obtained by M. Raczkowski. Figure published in ref. $\left[\mathrm{LMP}^{+} 16\right]$.

\section{Obtaining the Phase Diagram}

Here, the numerical results that lead to the above phase diagram are described. Metal and insulator are again identified by looking at the electron filling $\langle n\rangle$ as a function of chemical potential $\mu$. It shows a plateau at half-filling for large Coulomb repulsion $U$, which corresponds to a vanishing charge compressibility $\kappa=-\frac{\partial\langle n\rangle}{\partial \mu}$ and indicates an insulator, whereas, for a metal, no plateau occurs.

Self-Energy Functional. Furthermore, VCA provides the possibility of identifying and tracing competing phases by analyzing the self-energy functional $\Omega\left(\mu, \mu^{\prime}, V\right)$. Figure 4.7 shows the self-energy functional $\Omega$ as a function of hybridization $V / t$ for two different interchain hopping strength that correspond to the different regions of the phase diagram. For all values of $\Omega(V)$, the functional has already been optimized with respect to $\mu$ and $\mu^{\prime}$. The stationary points of $\Omega\left(\mu, \mu^{\prime}, V\right)$ indicated by arrows are hence maxima with respect to $\mu$ and $\mu^{\prime}$, but minima with respect to hybridization strength $V$. For small interchain coupling $t_{\perp} / t=0.2$, one cannot resolve two disjoined SEF minima and the value of 


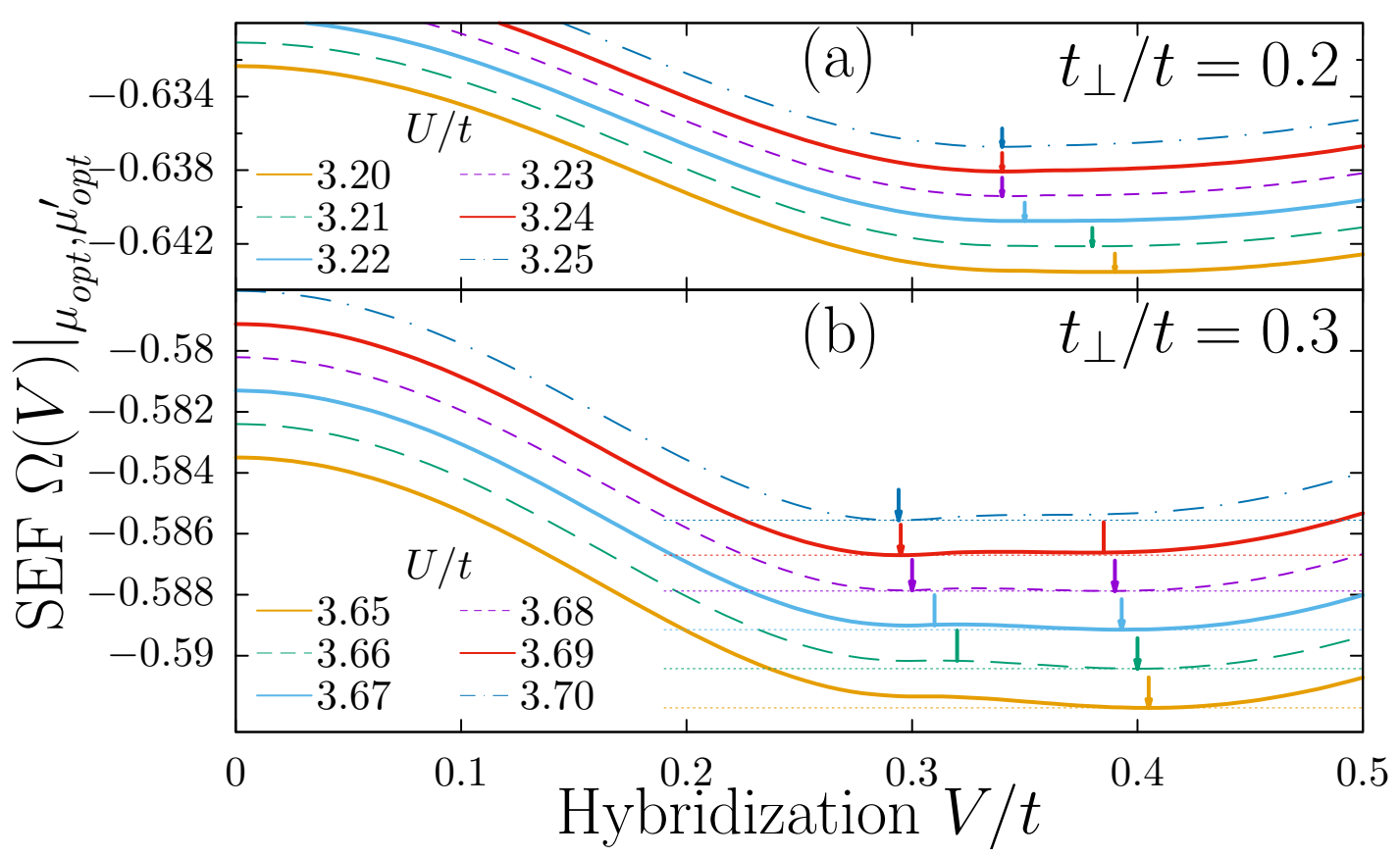

Figure 4.7: VCA self-energy functional $\Omega$ in the proximity of the MIT as a function of hybridization $V / t$ for (a) $t_{\perp} / t=0.2$ and (b) $t_{\perp} / t=0.3$. In (b) stable minima are indicated by thick arrows; thinner ones mark unstable solutions. Figure published in ref. [LMP $\left.{ }^{+} 16\right]$.

$V$ is thus expected to change continuously across the critical interaction $U_{c}$; see thick arrows in figure $4.7(\mathrm{a})$. In contrast, for $t_{\perp} / t \gtrsim 0.3$, the SEF has four saddle points, of which two (indicated by arrows) correspond to stable phases close to the phase transition: one corresponding to the metallic, the other to the insulating solution. The existence of two distinct minima in the SEF landscape shown in figure 4.7(b) results in a jump of hybridization $V$ when tuning across $U_{c}$, and thus signals the first-order nature of the MIT.
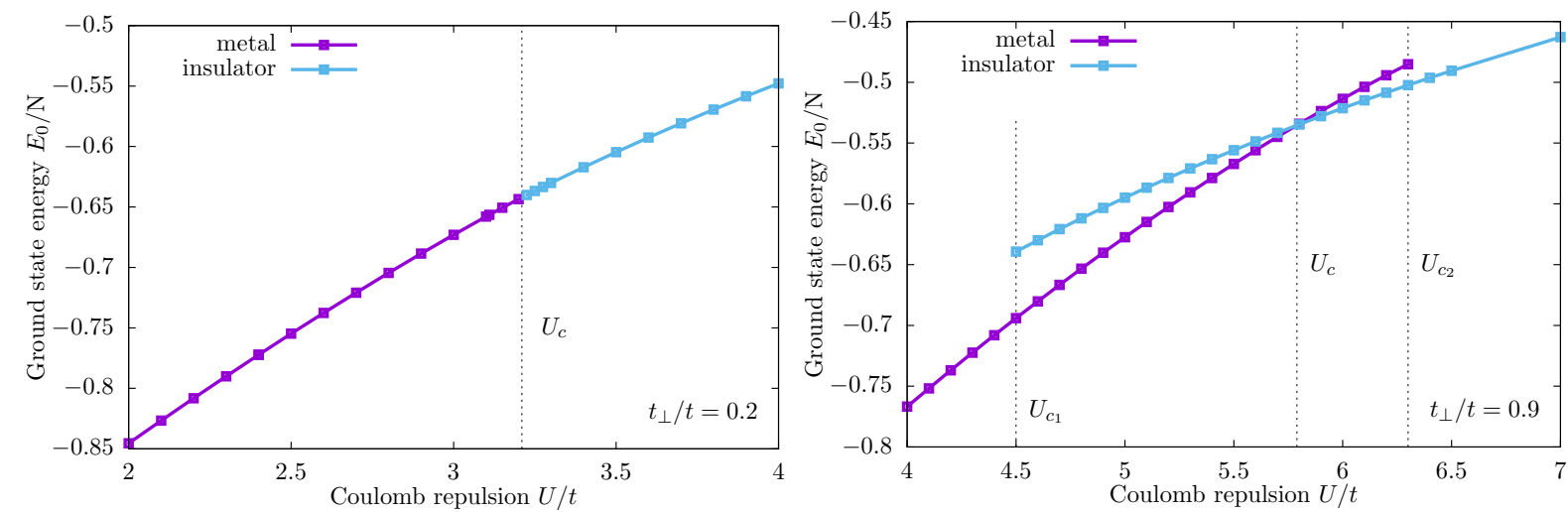

Figure 4.8: Ground-state energy $E_{0}$ as a function of interaction strength $U / t$ for $t_{\perp} / t=0.2$ (left panel) and $t_{\perp} / t=0.9$ (right panel). For large interchain hopping, the energies of the insulating and metallic solutions cross at $U_{c}$, which leads to a kink in the ground-state energy and indicates the discontinuous transition. At small interchain hopping, it is not possible to identify a coexistence region anymore and it is barely possible to make a statement on the absence or existence of a kink at $U_{c}$. 
Ground-State Energy $E_{0}$. Next, the focus is set on the ground-state energy $E_{0}$ at the stationary point(s). Figure 4.8 shows $E_{0}$ as a function of interaction strength $U / t$ for two different values of the interchain hopping strengths. In case of small $t_{\perp} / t=0.2$, the system is insulating down to the critical interaction strength and metallic for $U<U_{c}$. The ground-state energy seems to be a smooth curve without distinct kink at the transition, which indicates a continuous transition. Specifically, no insulting (metallic) solution is found for $U<U_{c}\left(U>U_{c}\right)$, as can be seen in the self-energy functional (top panel of figure 4.7).

In contrast, the quasi-2D region shows a clear kink in the ground-state energy $E_{0}$, see right panel of figure 4.9 for $t_{\perp} / t=0.9$. It arises from a level crossing of the insulating and metallic solutions, which both exist in a finite interaction range $U_{c_{1}}<U<U_{c_{2}}$. Following the solution of lowest energy across the transition leads to a kink in $E_{0}$ and indicates a discontinuous transition. Although a weak kink in $E_{0}$ is also resolved for intermediate values of $t_{\perp}$, the coexistence region shrinks when reducing $t_{\perp} / t$ and finally vanishes at $t_{\perp}^{c} / t \simeq 0.2$.

Double occupancy. Finally, the double occupancy $d$ is investigated. It is obtained as the derivative of the grand potential $\Omega$ with respect to Coulomb repulsion $d=\frac{\partial \Omega}{\partial U}$. The kink in energy $E_{0}$ for large and intermediate $t_{\perp} / t$ gives rise to a jump in the double occupancy $d$ at $U_{c}$, as shown in Fig. 4.9. It also exhibits hysteresis in the region with two solutions, as expected for a first-order transition. When reducing the interchain hopping, both the size of the jump in double occupancy at $U_{c}$ and the hysteresis region shrink. At $t_{\perp} / t$, it is already barely possible to identify a hysteresis region and at $t_{\perp}^{c} / t$, no jump is visible anymore. For intermediate and large $t_{\perp}$, the metallic and insulating solutions both exist in close vicinity of the transition and the jump can be read off immediately, see top right panel of figure 4.9 In the case of small $t_{\perp}$, it is necessary to fit double occupancies of the metallic and insulating solutions to read off the jump at the transition (see inset in Fig. 4.9). Although an exponentially small jump for $t_{\perp} / t \lesssim 0.2$ can still not be ruled out in this way, a fit of the jump sizes for $t_{\perp} / t>0.2$ agrees with a vanishing jump size for small $t_{\perp}$.

The absence of a jump in double occupancy and a single minimum in the self-energy functional yield strong evidence for the continuous nature of the metal-insulator transition at small interchain hopping strengths $t_{\perp}<t_{\perp}^{c}$. For intermediate and large $t_{\perp}$ the first order transition is clearly visible.

\section{\begin{tabular}{l|l} 
Comparison to Cellular Dynamical Mean-Field Theory & 4.2 .3
\end{tabular}}

The CDMFT results discussed here to compare with the VCA results at zero temperature were obtained by M. Raczkowski. Within CDMFT with a QMC solver, the model system can be analyzed at finite temperatures $T$. Before turning to the finite-temperature consequences of the continuous transition seen at $T=0$, the results of CDMFT at the lowest reached temperature $T=t / 40$ are compared with the VCA results. Figure 4.11 shows the double occupancy $d$ both for VCA (lines) and CDMFT (points) in the center panel. The qualitative behavior is the same in both techniques: Within finite-temperature CDMFT, the double occupancy $d=\frac{1}{N_{c}} \sum_{\boldsymbol{i}}\left\langle n_{\boldsymbol{i} \uparrow} n_{\boldsymbol{i} \downarrow}\right\rangle$ shows a jump in the quasi-2D regime, too, which gradually decreases when reducing $t_{\perp}$. Finally, it converts into a crossover at $t_{\perp}^{c} / t=0.2$. 
However, the values of $U_{c}$ and the height of the jump in $d$ at the transition are similar but do not coincide. The discrepancy in $U_{c}$ can be explained by taking into account that both techniques are biased into different directions when it comes to determining $U_{c}$ : When reducing the temperature within CDMFT, the value of $U_{c}$ becomes smaller, as can be seen from figure 4.10, where the double occupancy is shown as a function of interaction $U$ and temperature $T$. Therefore, the $U_{c}$ value for zero temperature within CDMFT is expected to be smaller than the one at $T=t / 40$ shown in the plot. For VCA on the other side, it is known that using only one bath site per correlated site slightly favors the metallic region $\left[\mathrm{BKS}^{+} 09\right]$. This bias towards the metallic phase results in a too large critical interaction strength $U_{c}$.

The finite temperature within CDMFT also leads to a reduction of $\Delta d$, which explains the difference to zero-temperature VCA. However, the effect of these thermal fluctuations becomes important even at $T=t / 40$ when turning to small interchain hopping. As there
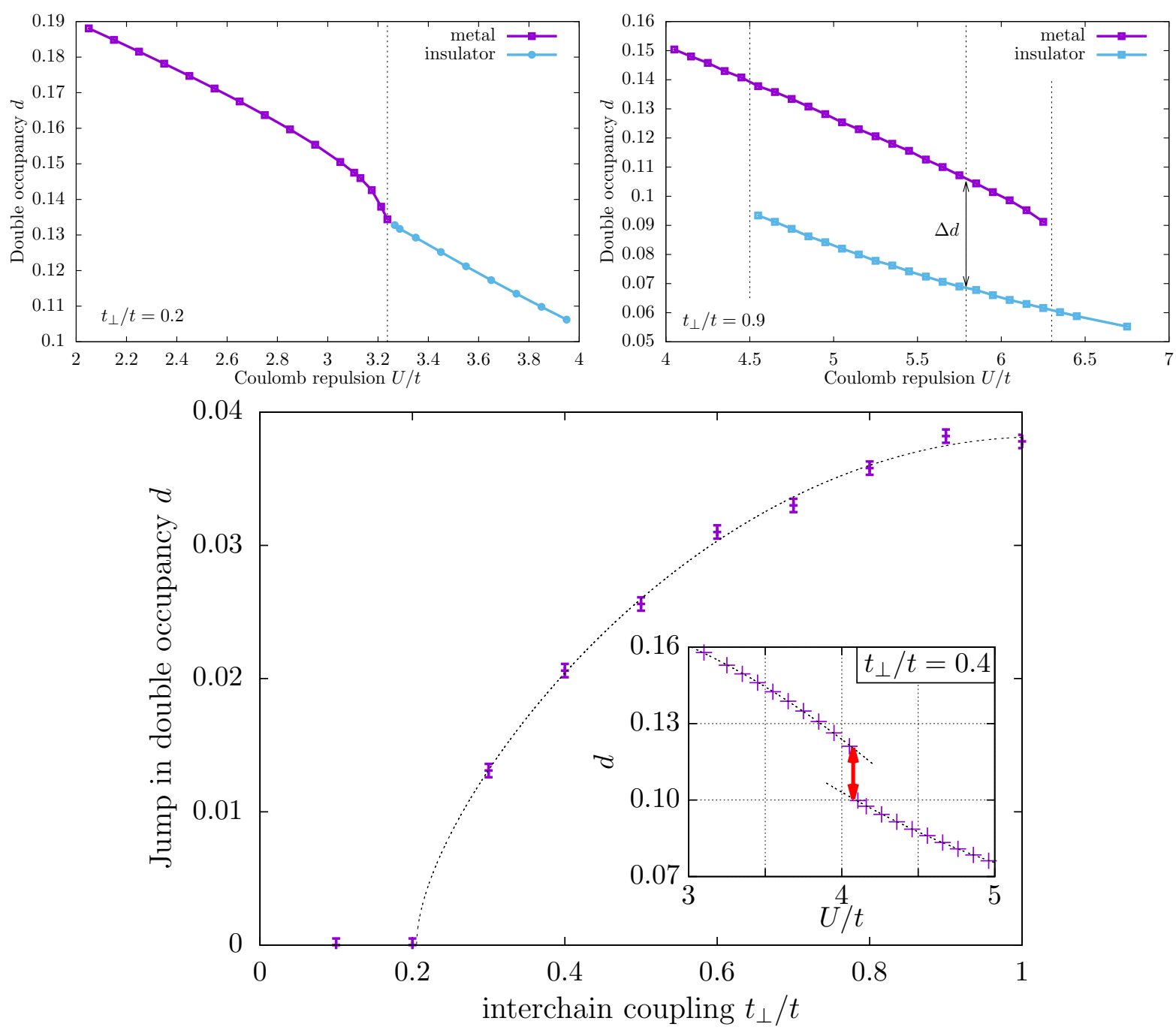

Figure 4.9: Double occupancy $d=\partial \Omega / \partial U$ as a function of interaction strength $U / t$ for $t_{\perp} / t=0.2$ (top left panel) and $t_{\perp} / t=0.9$ (top right panel). For $t_{\perp}>0.2$ the double occupancy shows hysteresis between $U_{c_{1}}$ and $U_{c_{2}}$ and a jump in $d$ at $U_{c}$. At small $t_{\perp}$, both hysteresis and a jump are absent. Bottom panel: Size of the jump in double occupancy at the MIT. The dashed line is a rough fit and only meant as a guide to the eye. Bottom panel published in ref. [LMP $\left.{ }^{+} 16\right]$. 

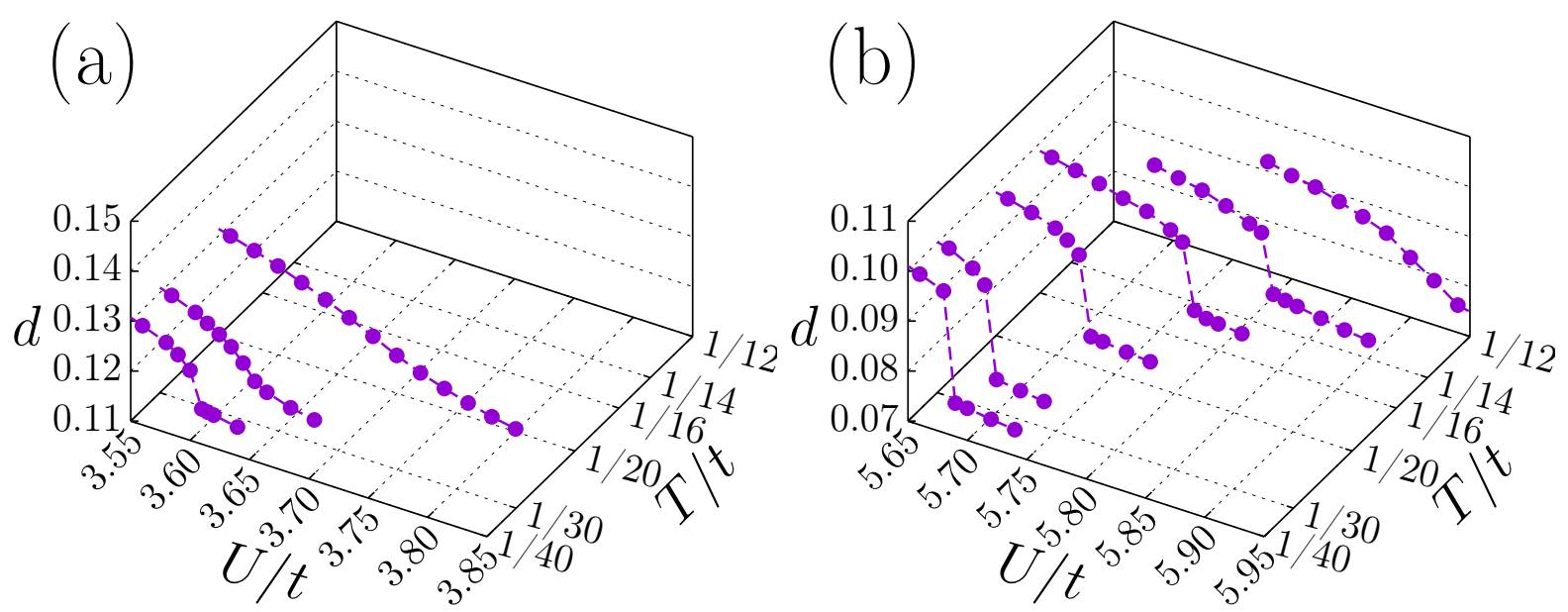

Figure 4.10: Double occupancy $d$ as a function of interaction $U / t$ obtained in CDMFT for (a) $t_{\perp} / t=0.3$ and (b) $t_{\perp} / t=0.9$. The low- $T$ jump in $d$ signaling the first-order MIT turns into a crossover above the critical end point $T_{c}$. CDMFT data obtained by $M$. Raczkowski. Figure published in ref. $\left[\mathrm{LMP}^{+} 16\right]$.

is no jump in $d$ within CDMFT for small interchain couplings $t_{\perp} / t \lesssim 0.2$ down to the lowest accessible temperature $T=t / 40$, the VCA data at $T=0$ is crucial to demonstrate the existence of quantum criticality at small $t_{\perp}$.

Within CDMFT, one can estimate the $t_{\perp}$ dependence of the critical-endpoint temperature $T_{c}$, where thermal fluctuations turn a discontinuous transition at $T<T_{c}$ into a continuous crossover at $T>T_{c}$. This estimate of $T_{c}$ at a given $t_{\perp}$ was obtained by monitoring $d$ as a function of $U / t$ at fixed temperature $T$, as shown in figure 4.10. The low- $T$ jump in $d$ signals the first-order MIT and remains up to $T_{c}$. For larger temperatures $T>T_{c}$ it turns into a smooth crossover. As shown in the left panel of figure 4.10, for small $t_{\perp} / t=0.3 \mathrm{a}$ smooth behavior in $d$ is already recovered at $T=t / 30$. In contrast, for $t_{\perp} / t=0.9$, the jump converts into a crossover at much higher temperature $T=t / 12$. By repeating the above analysis for intermediate values of $t_{\perp}$, the $t_{\perp}$ dependence of the critical temperature $T_{c}$ can be extracted (see the inset in Fig. 4.6).

The bottom panel of figure 4.11 shows the cluster spin susceptibility $\chi_{s}(\boldsymbol{q})$ at the AF wave vector $\boldsymbol{q}=(\pi, \pi)$ and at the vector $\boldsymbol{q}=(\pi, 0)$ for the CDMFT data at $T=t / 40$. It is defined via $\chi_{s}(\boldsymbol{q})=\frac{1}{N_{c}} \int_{0}^{\beta} d \tau \sum_{i \boldsymbol{j}} e^{i \boldsymbol{q} \cdot(\boldsymbol{i}-\boldsymbol{j})}\left\langle\boldsymbol{S}_{\boldsymbol{i}}(\tau) \boldsymbol{S}_{\boldsymbol{j}}(0)\right\rangle$. At the AF wave vector, the susceptibility shows a jump at the transition, which means that the level crossing in the ground state is also reflected in the spin sector. For small interchain hopping $t_{\perp} / t=0.2$, no clear distinction can be made between the response in $\chi_{s}(\boldsymbol{q})$ at $\boldsymbol{q}=(\pi, 0)$ and $\boldsymbol{q}=(\pi, \pi)$ wave vectors. This indicates that, at small $t_{\perp} / t$, remnant $1 \mathrm{D}$ effects indeed play an important role.

\section{Spectral Function and Fermi Surface Pockets}

To gain further insights into the microscopic origin of the continuous Mott transition for $t_{\perp} / t \lesssim 0.2$, the single-particle spectral function $A(\boldsymbol{k}, \omega)=-\frac{1}{\pi} \operatorname{Im} G(\boldsymbol{k}, \omega)$ is investigated, 


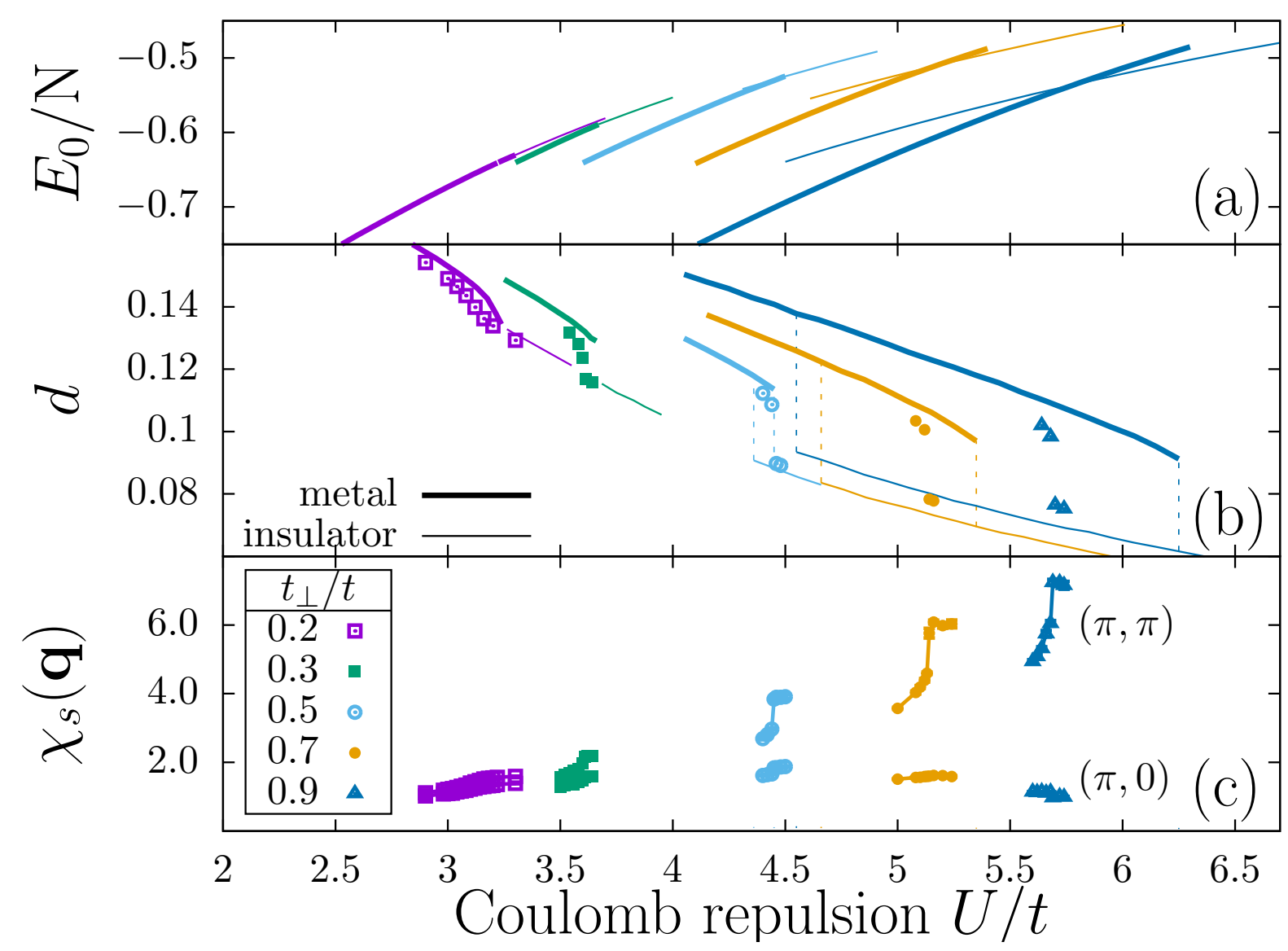

Figure 4.11: ((a) VCA ground-state energy $E_{0}$ as a function of Coulomb repulsion $U / t$. Filled (dashed) lines indicate metallic (insulating) solutions for $t_{\perp} / t=0.2,0.3,0.5,0.7$, and 0.9 (from left to right). (b) VCA double occupancy $d$ across the MIT at $T=0$; symbols stand for CDMFT results at $T=t / 40$. (c) Cluster spin susceptibility $\chi_{s}(\boldsymbol{q})$ within CDMFT at $T=t / 40$; the upper curves correspond to spin fluctuations at the AFM wave vector $\boldsymbol{q}=(\pi, \pi)$ and the lower ones to remnant $1 \mathrm{D}$ fluctuations at $\boldsymbol{q}=(\pi, 0)$. CDMFT data obtained by $M$. Raczkowski. Figure published in ref. $\left[\mathrm{LMP}^{+} 16\right]$.

where $G(\boldsymbol{k}, \omega)$ denotes the lattice Green's function.

In figure 4.12, the low-energy part of the spectral function is shown along a path in $k_{x^{-}}$ direction inside the first Brillouin zone around $(\pi / 2,0)$. For small interchain hopping $t_{\perp} / t=0.2$ (top panels in the figure), the spectral function intersects with the Fermi energy $\omega=0$ at two k-points, once with large, the other time with reduced weight. These intersections define the Fermi surface in the first Brillouin zone. As a result, the intersections form elliptic electron and hole pockets around the $\boldsymbol{k}=(\pi / 2,0)$ and $(\pi / 2, \pi)$ points. At $t_{\perp} / t=0.2$, the MIT happens at a critical interaction strength $U_{c} / t=3.24$. The figure shows the spectral function in the metallic phase (left panel), close to the transition (center panel) and in the insulating phase. While increasing the interaction strength, the spectral function smoothly moves to higher energies and the pocket structures at the Fermi surface only shrink first, then become very shallow and vanish at $U_{c}$.

In contrast, for larger $t_{\perp} / t=0.5$ (bottom panels), the volume reduction of the Fermi surface pockets is cut off by a first-order transition at $U_{c}=4.50$. Hence, the size of the pockets jumps abruptly from a finite size (center panel) to zero in the insulator (right 


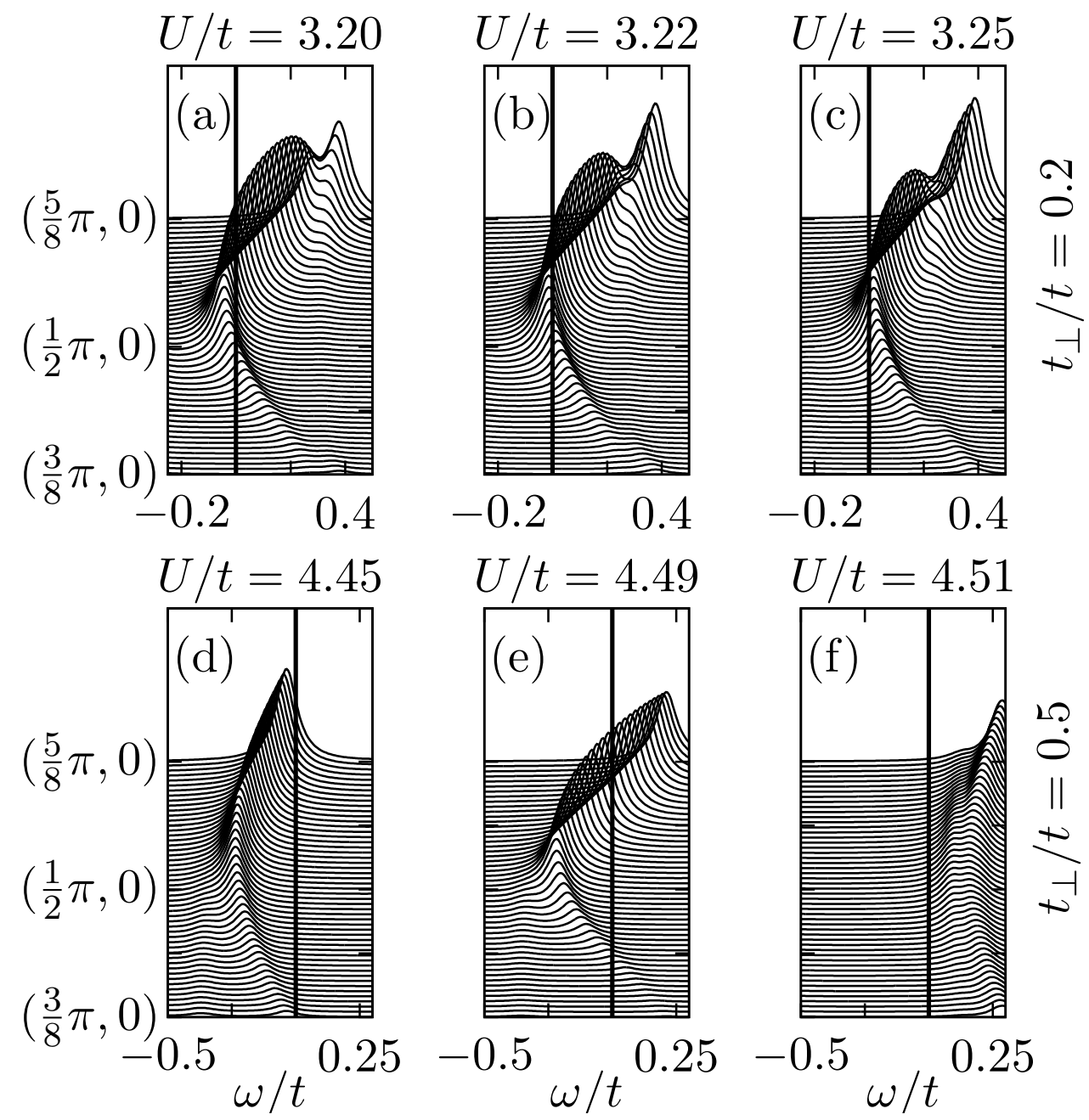

Figure 4.12: Low-energy part of the single-particle spectral function $A(\boldsymbol{k}, \omega+i \eta)$ for $t_{\perp} / t=0.2$ (top panels) and $t_{\perp} / t=0.5$ (bottom panels) obtained within VCA at $T=0, \eta=0.05$. The FS pockets are found (a),(d) for $U<U_{c}$ and (b),(e) at $U \lesssim U_{c}$; in the insulator at (c),(f) $U>U_{c}$, their disappearance signifies the vanishing of the FS, and hence the MIT. Figure published in ref. $\left[\mathrm{LMP}^{+} 16\right]$.

panel).

Figure 4.13 shows the evolution of $A(\boldsymbol{k}, \omega)$ upon increasing the interaction strength $U / t$ at fixed $t_{\perp} / t=0.2$. At small interaction strengths, the FS still follows the tight binding dispersion closely. When increasing $U$, the destruction of the FS starts at momenta $\boldsymbol{k}=(\pi / 2, \pm \pi / 2)$, where the interchain-hopping matrix elements vanish. This is in agreement with random-phase approximation studies [ET02, RSP11]. However, in contrast to a study on coupled spinless fermionic chains [BGBG06], which also showed pockets, a striking feature of the pockets here is their symmetric form. This symmetry can be ascribed to quasiparticle scattering off short-range $1 \mathrm{D}$ spin fluctuations with $\boldsymbol{q}=(\pi, 0)$. At intermediate interaction strengths, the main part of the FS carrying most of the quasiparticle weight still follows closely the noninteracting FS. When approaching the transition line, the pockets shrink and continuously vanish at $U_{c} / t=3.24$. This continuous vanishing of their volume implies the second-order nature of the MIT. It also offers a possibility to study the critical behavior of the transition: Since the inverse width of the hole or 
electron pockets defines a characteristic length scale, $\xi$, one should be able to extract the correlation length exponent, $\nu$ [IFT98]. A preliminary study on the critical behavior of the volume of the pocket as one approaches $U_{c}$ is shown in subsection 4.2.5.

Figure 4.14 shows the Fermi surface for different values of $t_{\perp} / t$ close to the critical value $U_{c}$. When comparing to the tight-binding limit, the main effect of the interaction is the formation of additional shadow-like bands, and the creation of gaps in the originally continuous structure. Both effects form the compensated metal structure of the FS with hole and electron pockets in the region $t_{\perp} / t<0.7$. However, the weight of the shadow-like bands decreases for larger interchain hopping. This is consistent with the assumption of the formation of shadow-like bands being a result of $1 \mathrm{D}$ fluctuations. For larger $t_{\perp} / t>0.7$, the pocket structures disappear and a conventional large Fermi surface is formed. In the case of a continuous transition for $t_{\perp} / t \leq 0.2$, the volume of the hole and electron Fermi pockets shrinks to zero at the critical interaction strength $U_{c}$.

So far, all plots on the spectral function and the emerging Fermi surface pockets were obtained from VCA results at zero temperature. Figure 4.15 shows a comparison between VCA data (top panels) and CDMFT spectra at $T=t / 40$ (bottom panels) for three different interchain hopping strengths. For the latter stochastic analytic continuation [Bea04] has been applied to the imaginary-time QMC data. It can be seen that both techniques give the same qualitative picture: Shallow FS pockets with similar weight on the main and the shadow-like band are found close to the transition for small $t_{\perp} / t=0.2$. At $t_{\perp} / t=0.3$, the Fermi-surface pocket size is finite at the transition and both sides of the pocket already show an appreciable asymmetry in the weights, which can be read off as the peak heights of the two intersections of $A(\mathbf{k}, \omega+i \eta)$ and $\omega=0$.
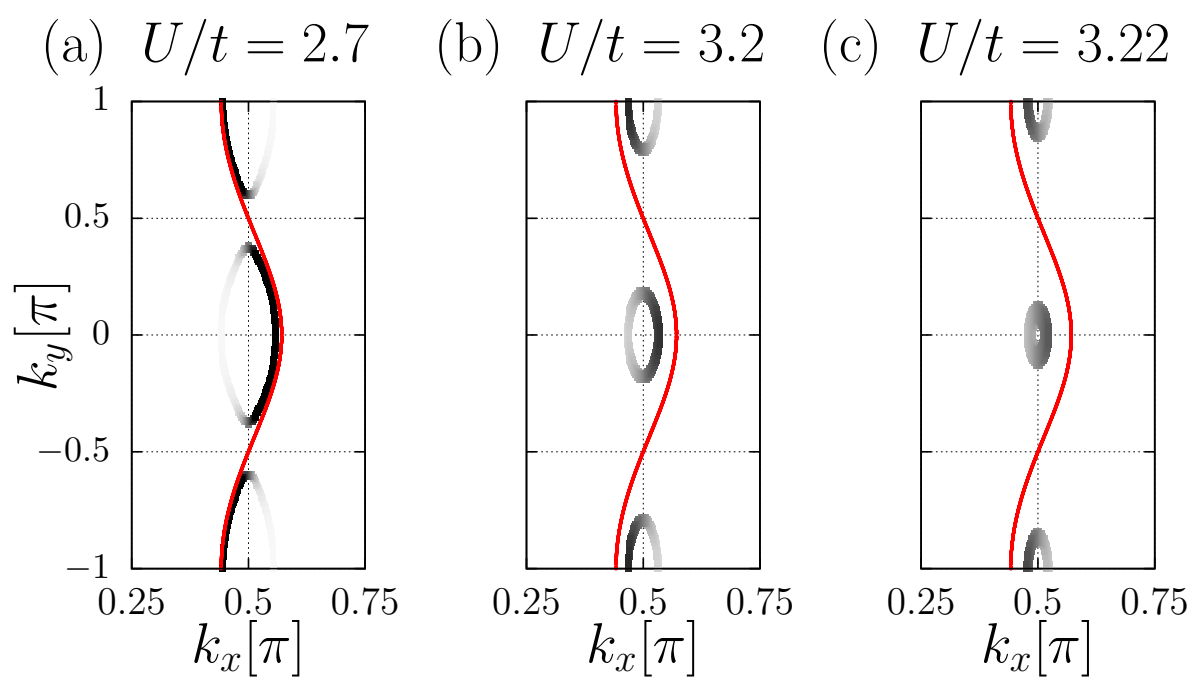

Figure 4.13: Evolution of the FS with electron and hole pockets (see the text) for $t_{\perp} / t=0.2$ when approaching $U_{c} / t \simeq 3.22$ from below: (a) $U / t=2.7$, (b) $U / t=3.2$, and (c) $U / t=3.22$. Red solid lines show the noninteracting dispersion. Figure published in ref. [LMP $\left.{ }^{+} 16\right]$. 


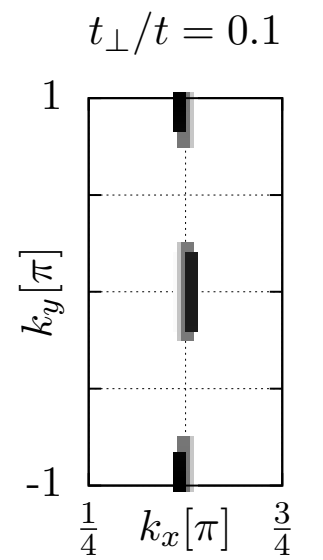

$$
t_{\perp} / t=0.2
$$

$$
t_{\perp} / t=0.3
$$

$$
t_{\perp} / t=0.5
$$

$$
t_{\perp} / t=0.9
$$
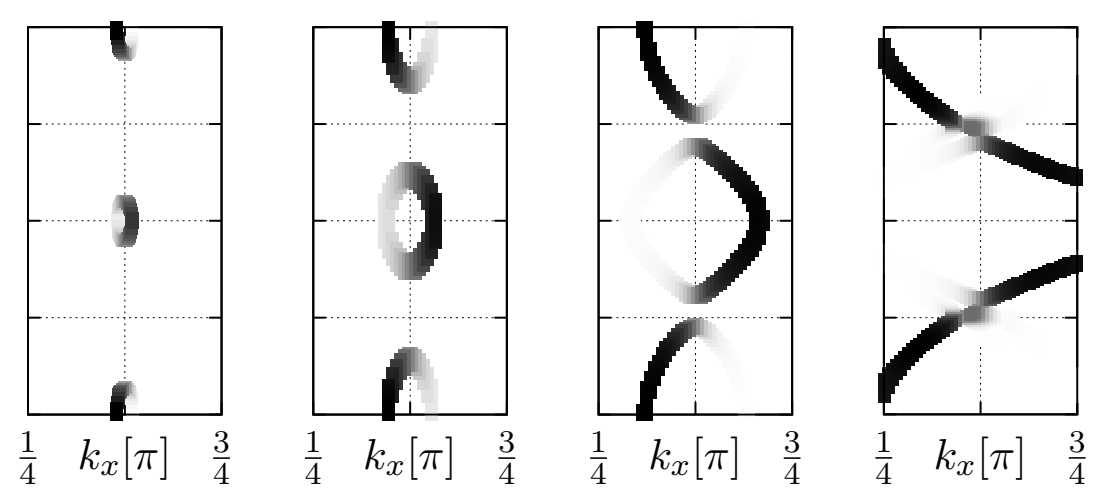

Figure 4.14: Fermi-surface topology for several values of the interchain coupling $t_{\perp} / t$ close to the critical interaction strength $U_{c}$. For $t_{\perp} / t>0.7$ the compensated metal structure of the FS with hole and electron pockets disappears going over to a conventional large Fermi surface. Figure published in supplemental material to ref. $\left[\mathrm{LMP}^{+} 16\right]$. 


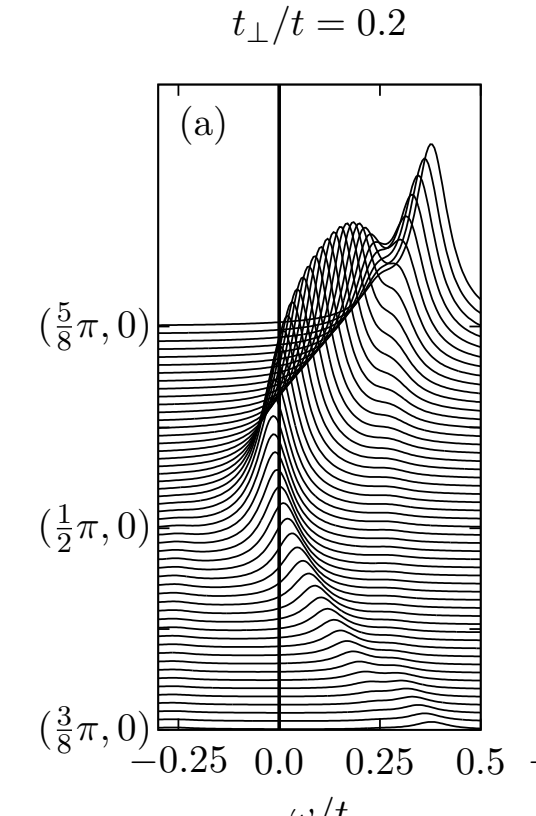

$t_{\perp} / t=0.3$

$t_{\perp} / t=0.5$
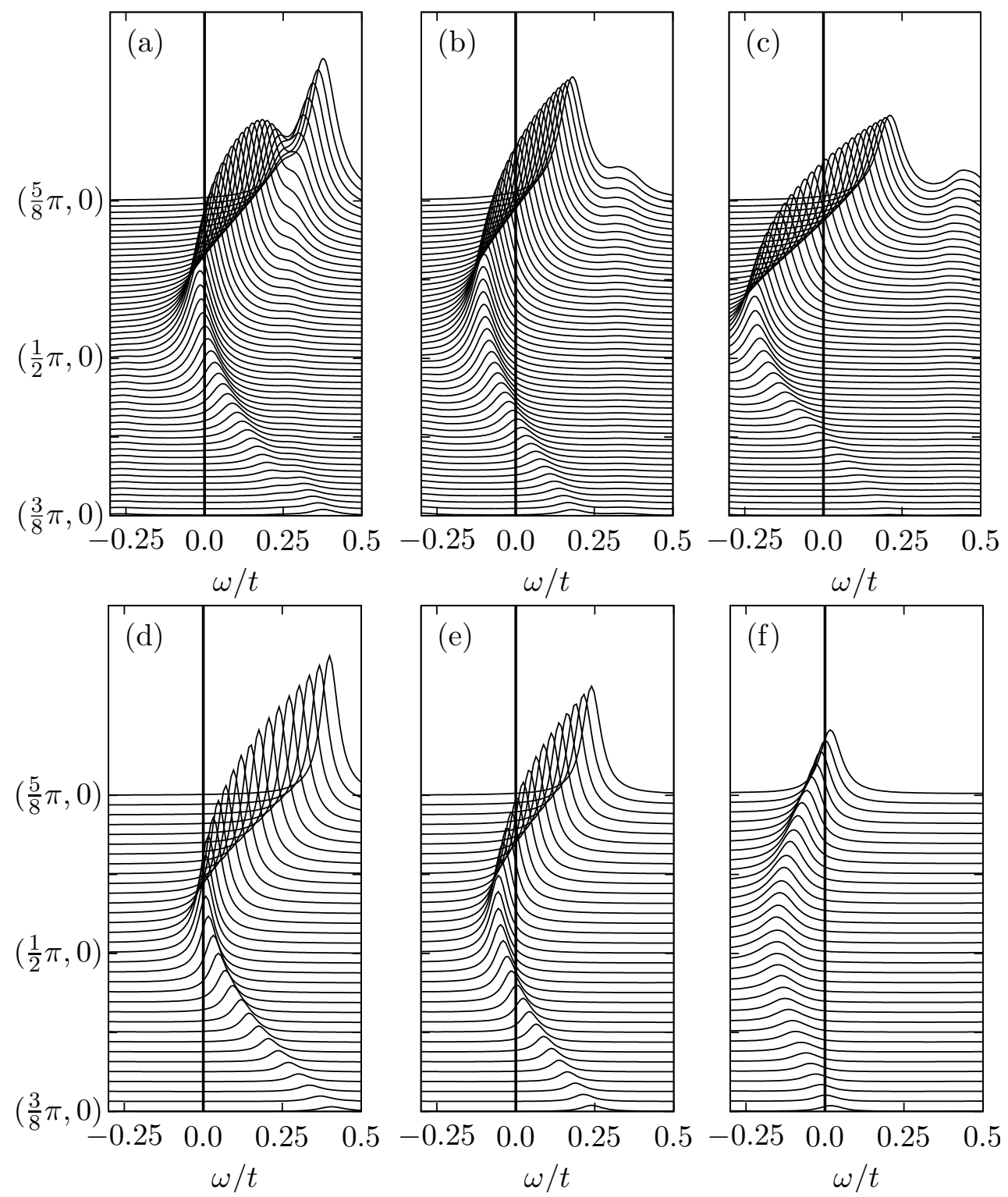

Figure 4.15: Low-frequency part of the single-particle spectral function $A(\boldsymbol{k}, \omega+i \eta)$ in the metallic phase close to the critical interaction $U_{c}$ for different $t_{\perp}$ obtained within VCA at $T=0$ (top) and CDMFT at $T=t / 40$ (bottom): (a,d) $t_{\perp} / t=0.2$, (b,e) $t_{\perp} / t=0.3$, and (c,f) $t_{\perp} / t=$ 0.5. In VCA, small broadening $\eta=0.05$ was used; stochastic analytic continuation [Bea04] of the imaginary-time QMC data was applied within CDMFT. CDMFT data obtained by $M$. Raczkowski. Figure published in supplemental material to ref. [LMP $\left.{ }^{+} 16\right]$. 


\section{Critical Exponents at the Mott Transition $\mid 4.2 .5$}

Albeit all the advantages of VCA, the fate of mean-field-like techniques, to which up to some extend one has to count the variational cluster approximation, too, is their incapability to obtain the correct critical exponents of the physical system that is approximated. For quantum cluster techniques like VCA or CDMFT the correlation length is exactly taken into account only within the cluster. Beyond this length scale, the mean-field(like) construction leads to mean-field exponents. This limitation becomes important once divergent correlation lengths, as for example in second-order phase transitions, are considered. Still, one can ask the question, whether one can learn something with respect to criticality from quantum cluster methods like VCA.

Indeed, aspects of the data can already provide an understanding of the quantum critical behavior at $T=0$. In the following, two different quantities are analyzed in order to gain insights into critical exponents. The first paragraph shows how the size of the Fermi surface pockets can be used to make statements on the correlation length exponent $\nu$. Afterwards, hyperscaling is assumed and the double occupancy is used to extract the dynamical exponent $z$ by assuming a mean-field exponent of $\nu=1 / 2$.

Characteristic length scale of the pockets. To quantify the interaction-driven renormalization of the FS warping for $t_{\perp} / t=0.2$, the left panel of figure 4.16 shows the width of the electron pocket as a function of $U / t$. The change in the nature (second vs. first order) of the MIT with increasing interchain coupling is also seen in the $t_{\perp}$ dependence of the pocket width as a kink at $t_{\perp} / t=0.2$. It marks the crossover between the regime where the volume of hole and electron Fermi pockets vanishes continuously at the second-order Mott transition and the region where the volume reduction of the pockets is cut off by a first-order transition. For $U \ll U_{c}$, the main part of the FS carrying most of the quasiparticle weight closely follows the tight-binding dispersion. Increasing the interaction strength results in a measurable deviation from the tight-binding disper-
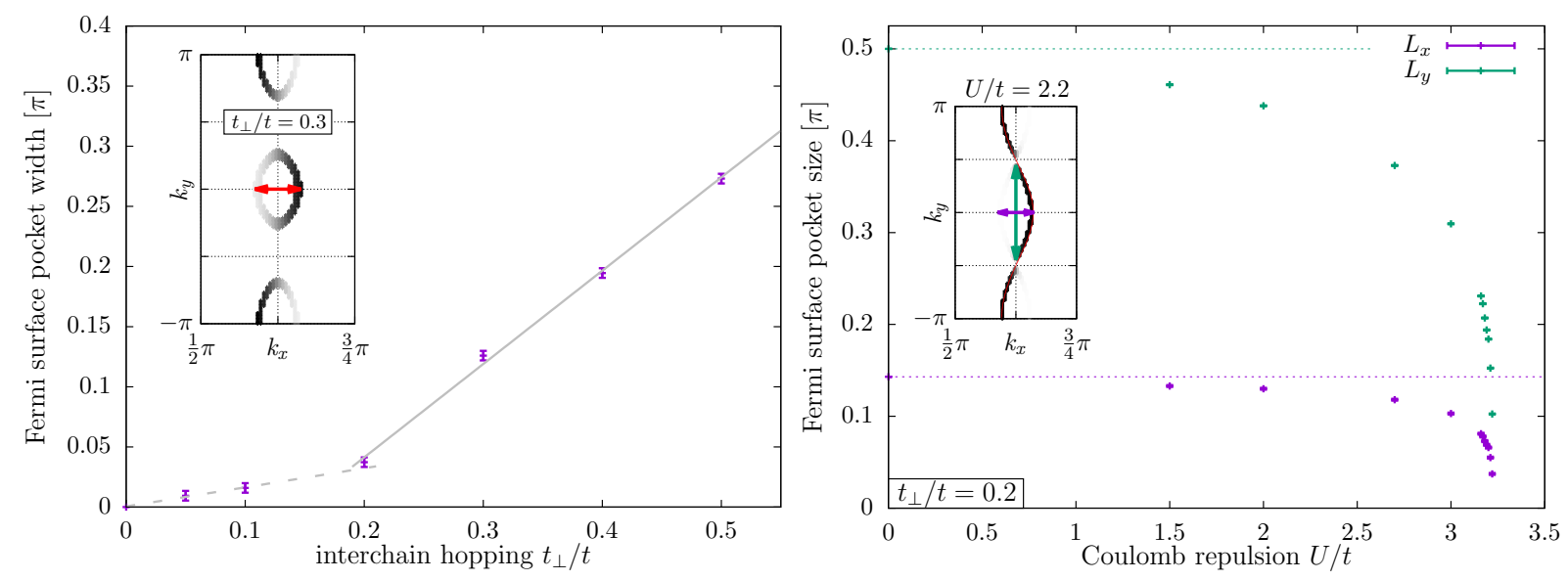

Figure 4.16: (Figures published in supplemental material to ref. $\left[\mathrm{LMP}^{+} 16\right]$ ) Left panel: Width of the electron pocket as a function of $t_{\perp} / t$ at $U=U_{c}-0.01$. The location of the kink agrees with the change in the nature of the MIT. Right panel: Size of the electron pocket for $t_{\perp} / t=0.2$ as a function of $U / t$ (top panel); the length of the pocket $L_{y}$ has been divided by a factor of two. The tight-binding limit $U \rightarrow 0$ is indicated by the dashed lines. 

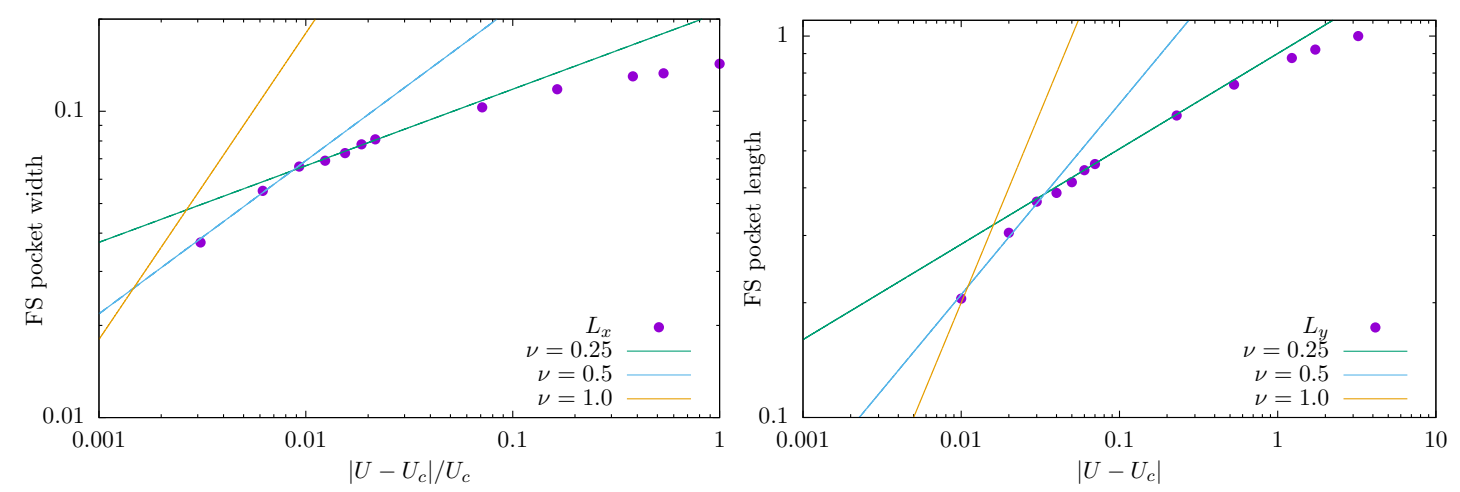

Figure 4.17: Width $L_{x}$ (left) and lenght $L_{y}$ (right) of the pockets for $t_{\perp} / t=0.2$ and $U_{c} / t=$ 3.23. Close to $U_{c}$ the data seems to agree with an exponent of 0.5 , whereas an exponent of 0.25 fits well far off the transition. However, quality and number of accessible points is not sufficient to make a definite statement.

sion. There, the continuous metal-to-insulator quantum phase transition corresponds to the shrinkage of the volume of the Fermi pockets when approaching the critical point $U_{c}$. Since the inverse width of the hole or electron pockets defines a characteristic length scale, $\xi$, the correlation length exponent $\nu$ can be extracted [IFT98].

However, it is difficult to obtain a precise value of the electron- and hole-pocket sizes, especially close to the phase transition, where the pockets vanish. As a consequence, only few points are available to fit the data and to extract the exponent $\nu$, see the right panel of figure 4.17.

Far away from the transition, the available data both for the width and the length of the pockets suggests an exponent of $\nu=0.25$, but close to $U_{c}$ rather an exponent of $\nu=0.5$ is possible. Larger critical exponents are not supported by the data, but a definite statement on the precise value of $\nu$ can be barely made from this analysis.

One of the drawbacks of taking the size of the Fermi-surface pockets are the additional errors that enter the calculation by reading off quantities from the broadened spectral function, which is based on the reperiodized Green function and a discretization of the Brillouin zone. A more direct way of accessing critical exponents consists in studying the double occupancy $d$ and is presented in the following paragraph. In order not to confuse it with the dimension $d=2$ of the system, the double occupancy is in the remainder of this chapter denoted with a capital letter $(D)$.

Hyperscaling and double occupancy. Under the assumption of hyperscaling, see, e.g., M. Imada et al. [IFT98], the singular part of the free energy should scale as,

$$
f \propto\left|U-U_{c}\right|^{\nu(d+z)}
$$

where $d=2$ is the dimension. Since the double occupancy reads

$$
D=\partial f / \partial U
$$

it should scale as

$$
D \propto\left|U-U_{c}\right|^{\nu(d+z)-1}
$$

This relation provides an opportunity to extract the dynamical exponent $z$. If the transition point $U_{c}$ and the corresponding double occupancy $D_{c}$ is determined precisely, this 

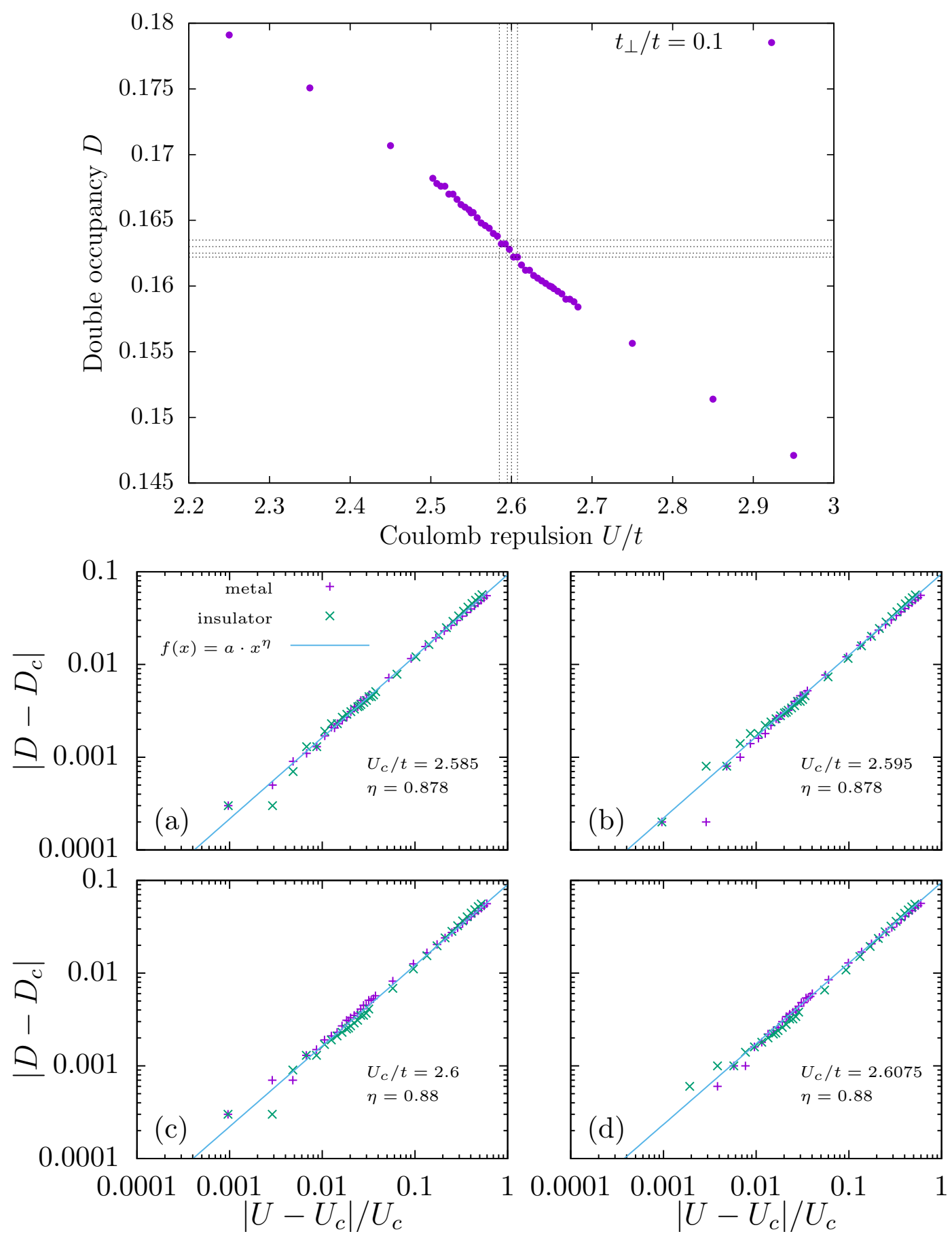

Figure 4.18: Top panel: Double occupancy $D$ as a function of $U / t$ for $t_{\perp} / t=0.1$ using VCA at $T=0$. Bottom panel: The data of both the metal and the insulating solution are fitted with the function $f(x)=a \cdot x^{\eta}$ after substracting the constant $D_{\text {c }}$ indicated by dashed lines in the top panel for different $U_{c} / t$.

allows for a scaling of $\left|D-D_{c}\right|$ with $\left|U-U_{c}\right|$, down to values close to the transition.

As the double occupancy changes continuously at $U_{c}$ and as it is already obtained as a numerical derivative of the self-energy functional $\Omega$ with respect to the Coulomb repulsion $U$, a precise determination of $U_{c}$ is challenging. As can be seen in figure 4.18, different 
values of $U_{c}$ are conceivable and also lead to qualitatively similar double-logarithmic plots of $\left|U-U_{c}\right|$ against $\left|D-D_{c}\right|$. However, in all four cases shown in the figure, the data collapses onto one line both for the insulating and the metallic side as it is expected from hyperscaling theory. The analysis of the double occupancy at $t_{\perp} / t=0.1$ and close to $U_{c}$ hence yields the fitted exponent around 0.88. Considering that cluster methods neglect critical spatial fluctuations $\left[\mathrm{DAH}^{+} 04\right]$, it is reasonable to assume a mean-field value of $\nu=1 / 2$, which in turn allows one to extract the estimate of $z \simeq 2 * 0.88=1.76$.

Thus, this preliminary study of the critical exponent indicates that the MIT might fall into the universality class of the transition between the band insulator and a metal with a small Fermi surface, i.e. $\nu=1 / 2$ and $z=2$, such that $D \propto\left|U-U_{c}\right|$, cf. [IFT98]. However, in order to make a definite statement, it is necessary to elaborate on this issue along the whole quantum critical line below $t_{\perp} / t=0.2$. This as well as an investigation of the influence of finite-size effects on the extraction of critical exponents is left for future studies. 


\section{Mott Transition into the Antiferromagnetic Phase}

In the previous section, the focus was set on the paramagnetic case. However, for the isotropic frustrated Hubbard model on a square lattice small frustration still leads to antiferromagnetic order at strong coupling. Only at comparably strong frustration, the antiferromagnetic long-range order on the insulating side of the metal-insulator transition is lost [NSST08, YSEO13].

In this section, first results are shown for the antiferromagnetic phase. The first subsection briefly discusses how antiferromagnetism is included in the investigation of the Mott transition via VCA. For two different relative frustration strengths the emerging phase diagram is shown and discussed in the subsequent subsections. It is shown that a continuous Mott transition might even prevail for the antiferromagnetic case at small interchain hopping. Although the data discussed in this section suggest such a scenario, an exponential decay of the jump in double occupancy and staggered magnetization in this region indicating a weak first-order transition cannot be excluded.

\section{Preliminary Assessment of the Inclusion of Magnetism $\mid 4.3 .1$}

Variational cluster approximation allows for the investigation of magnetism via suitably chosen Weiss fields. A reference cluster, which includes additional non-interacting bath sites, offers two different ways of applying such Weiss fields. Either a staggered magnetic Weiss field is applied to the correlated cluster sites or it is applied on the bath sites only. In this section, the latter is done, as it has been shown that applying the Weiss field to the bath sites leads to better results in the large $U / t$-limit [Bal08]. Furthermore, this way of including symmetry breaking allows for a more direct comparison with techniques like CDMFT where the antiferromagnetism is also included via the bath.

In the following, two different relative next-nearest-neighbor hopping strengths $t^{\prime}=-t_{\perp} / 4$ and $t^{\prime}=-t_{\perp} / 2$ are investigated. Previous VCA studies on the isotropic frustrated Hubbard model on a square lattice indicate that a stronger frustration of $t^{\prime} \approx 0.8 t$ would be necessary to destroy antiferromagnetic order in the ground state [NSST08, YSEO13]. In

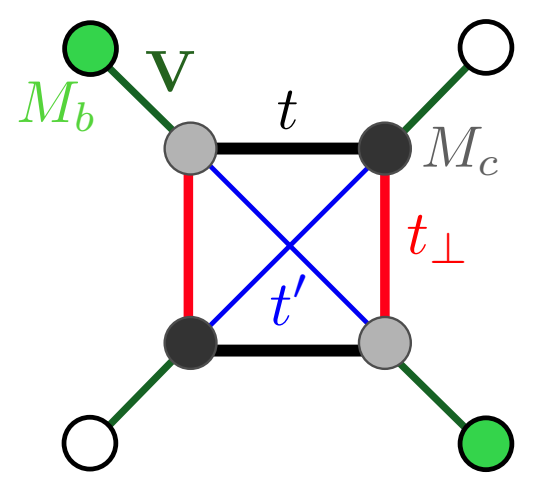

Figure 4.19: Reference cluster with additional Weiss fields on the correlated sites (field strength $M_{c}$ ) and on the bath sites (field strength $M_{b}$ ). 
these studies, even larger frustration leads to a collinear order along the $(\pi, 0)$ ordering vector. Although there no bath sites were included and the position of the transition $U_{c}$ therefore differs in the case studied here, similar behavior with respect to the formation of ordered phases can be expected. The left panel of figure 4.20 insinuates a similar behavior within VCA with bath sites included at strong coupling when including Weiss fields both on the cluster and on the bath. At $t^{\prime} / t \approx 0.8$ antiferromagnetic order is destroyed.

In the right panel, solutions corresponding to phases with collinear order are plotted in addition to the antiferromagnetic insulator and paramagnetic metal, which are discussed in this section. The phase with $(\pi, 0)$ order has been found in references [NSST08, YSEO13] for strong frustration. As can be seen from the plot, even for the larger frustration of the two values studied here, their energy is always higher than the one of the antiferromagnetic insulator. Therefore, these phases are not realized at the two frustration strengths $t^{\prime} / t_{\perp}$ used here and are not further investigated.

For both values of $t^{\prime} / t$ studied here, the solution at large interaction strengths corresponds to the antiferromagnetic insulator. In the following, it is analyzed how anisotropy influences the transition to a paramagnetic metal at smaller interaction. Therefore, the interchain hopping $t_{\perp} / t$ is again used as a continuously tunable control parameter.

\section{Phase Diagram $\mid 4.3 .2$}

The main findings of the preliminary study shown in this section are summarized in figure 4.21. In the left panel, the transition lines between the paramagnetic metal and the Mott-Heisenberg insulator are shown for both investigated frustration strengths. At large
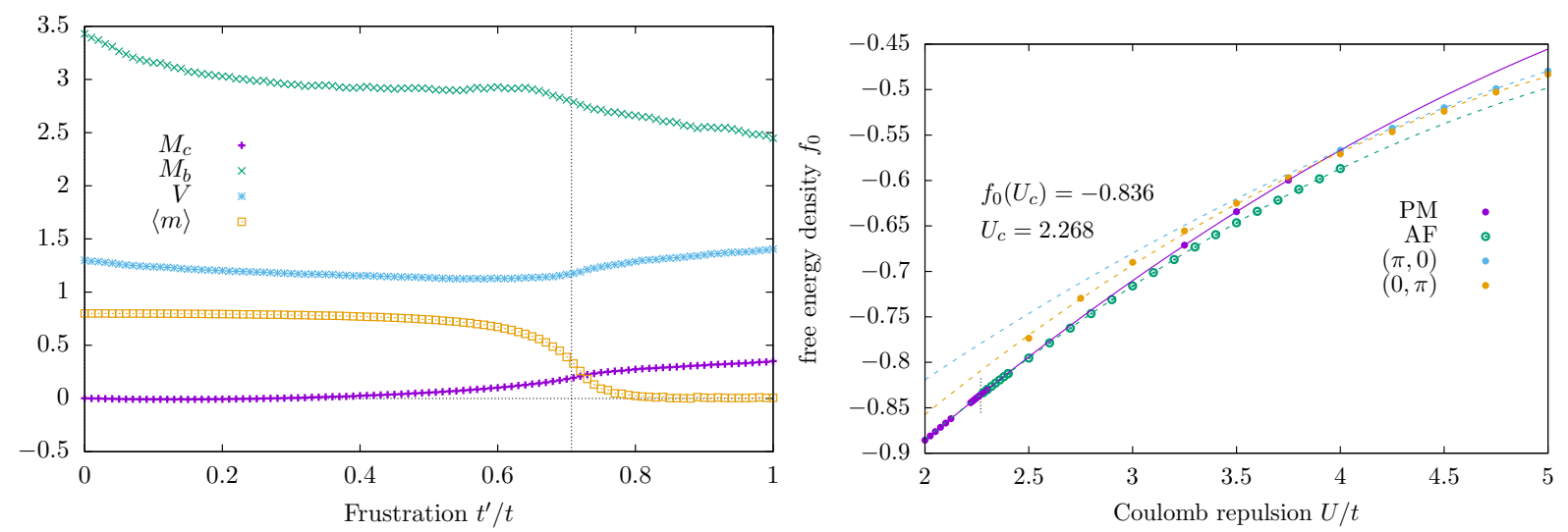

Figure 4.20: Left panel: Investigation of the isotropic Hubbard model $t_{\perp} / t=1.0$ as a function of next-nearest-neighbor hopping $t^{\prime} / t$ at strong interaction $U / t=8$. Staggered magnetic fields have been used both on the correlated sites $\left(M_{c}\right)$ and on the bath sites. The staggered magnetization indicates a vanishing of antiferromagnetism at $t^{\prime} / t \sim 0.7$. For $t^{\prime} / t \geq 0.8$, the system is not ordered antiferromagnetically anymore. Right panel: Ground-state energy $E_{0}$ as a function of interaction strength at $t_{\perp} / t=0.4, t^{\prime}=-t_{\perp} / 2$. In addition to the paramagnetic and antiferromagnetic phases discussed in this section, also phases with collinear-ordering vectors $(\pi, 0)$ and $(0, \pi)$ are shown. Their energy is always higher than the one of the antiferromagnetic insulator and they are hence not realized in this parameter regime of the model. Lines are fits to the data, but do not reflect the extend of the corresponding phases. 

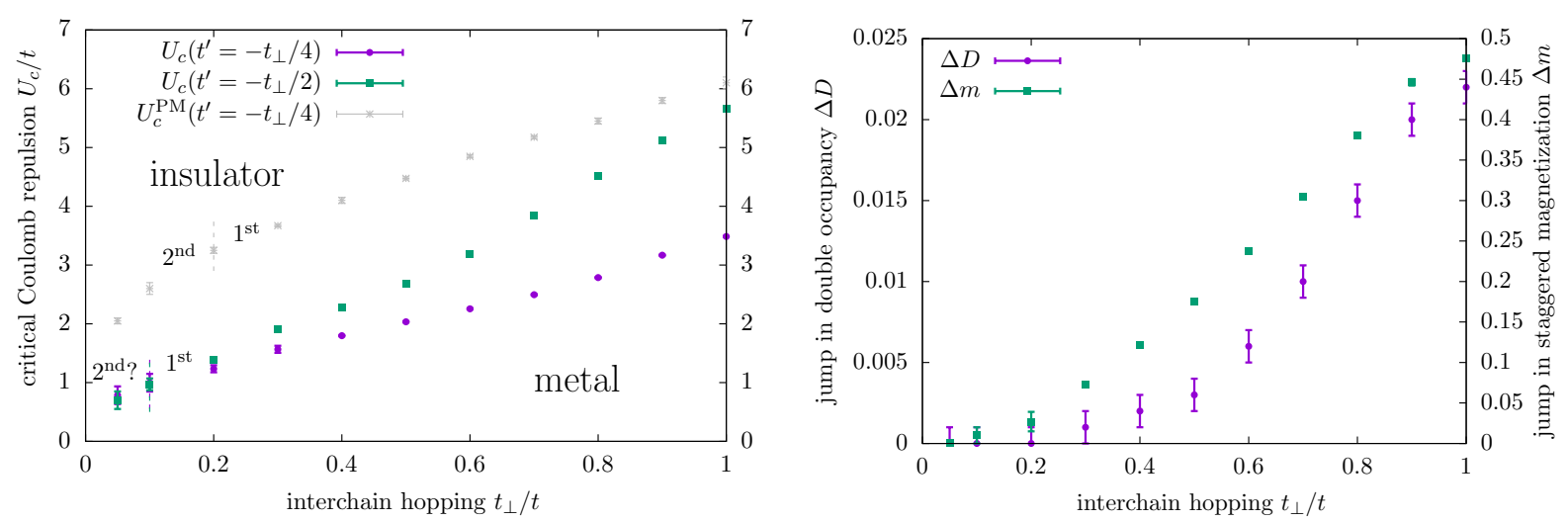

Figure 4.21: Critical interaction strength $U_{c}$ of the Mott transition between paramagnetic metal and Mott-Heisenberg insulator for two different frustration strengths. The right panel shows the jump in double occupancy $D$ and staggered magnetization $m$ for $t^{\prime}=-t_{\perp} / 4$. Absence of a jump in double occupancy suggests a continuous transition to be present at small interchain hopping, but extraction of a jump in magnetization is challenging and does not exclude a exponential decrease when approaching the decoupled case $t_{\perp} / t=0$.

and intermediate interchain hopping strengths $t_{\perp} / t$ the transition is of first order. This shows in a crossing of the energies of the two solutions. Following the solution of lowest energy results in a kink at the transition, which is accompanied by a jump in the double occupancy $D$ and in the staggered magnetization $m$. In the nearly isotropic regime $t_{\perp} / t \lesssim 1$, both solutions are found even at interaction strengths close to the transition $U \lesssim U_{c}$

The size of the jumps $\Delta D$ and $\Delta m$ at the transition reduces for smaller $t_{\perp} / t$. At small interchain hopping $t_{\perp} / t \lesssim 0.1$, no distinct jump is visible in neither of the two quantities, which suggests a continuous transition. For intermediate $t_{\perp} / t$, the jump size increases nearly linearly with the interchain hopping. However, when increasing the anisotropy, a tail is visible and it is barely possible to exclude the possibility of an exponentially small jump in $D$ and $m$ at small interchain hopping. Therefore, the regime of weakly coupled chains has to be studied further before making a definite statement on possible quantum critical behavior in this region.

The following subsection discusses some of the aspects that lead to the overall phase diagram in more detail.

\section{Obtaining the Phase Diagram $\mid 4.3 .3$}

The phase diagram shown in the previous subsection is based mainly on three quantities. Comparing the energy of the metal and the antiferromagnetic insulator allows to determine the crossing point $U_{c}$, which amounts to the critical interaction strength for the Mott transition. The double occupancy can be evaluated as the numerical derivative of the grand potential with respect to the interaction strength $U$ and is one of two indicators to determine whether the transition is discontinuous. In contrast to the previous section, where both the insulator and the metal had no long-range magnetic order, the staggered magnetization $m$ has a finite value in the Mott-Heisenberg insulator. The behavior of 


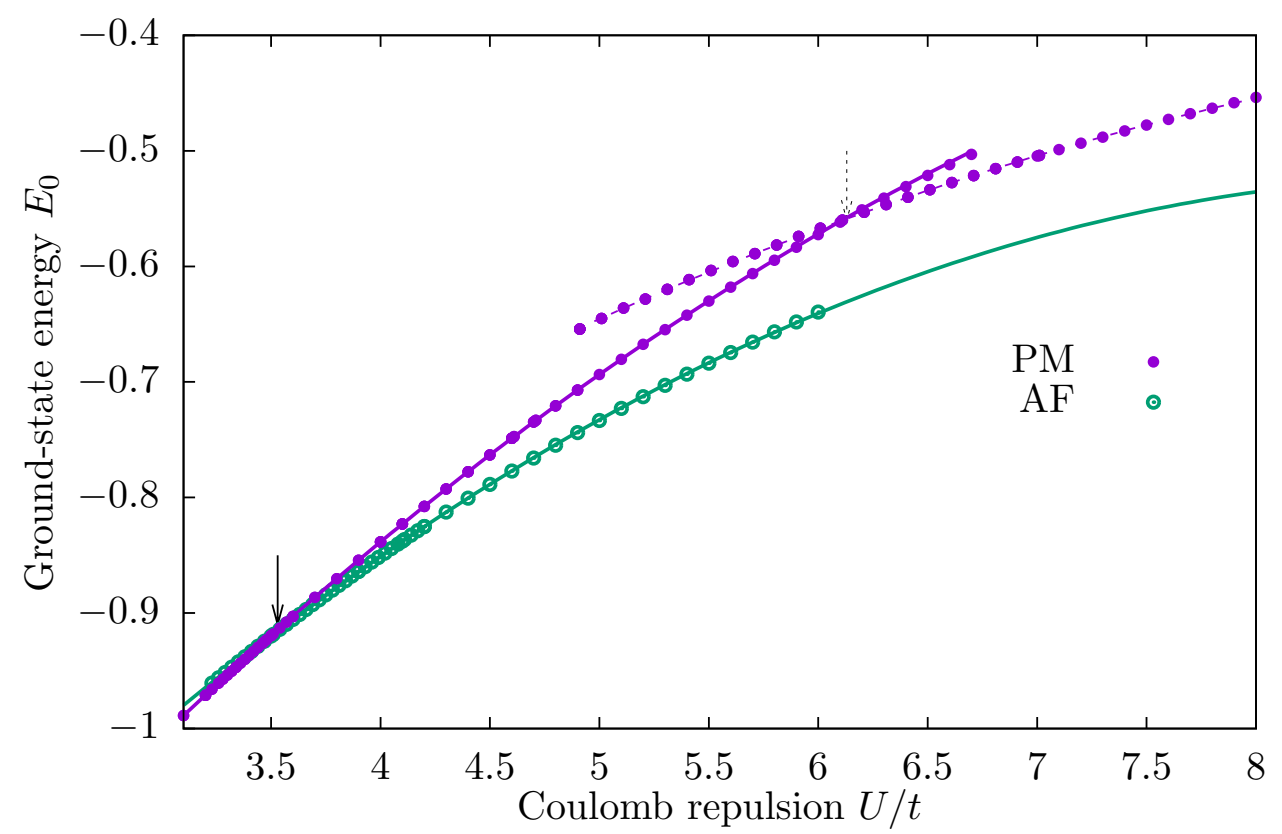

Figure 4.22: Ground-state energy $E_{0}$ as a function of interaction strength $U$ at $t_{\perp} / t=1.0$, $t^{\prime}=-t_{\perp} / 4$. At large interaction, the paramagnetic solution is insulating and changes for smaller $U$ to a paramagnetic metal at $U_{c}^{\mathrm{PM}}$, which is indicated by a dashed arrow. This transition has been studied in the previous section. The antiferromagnetic insulator is lowest in energy for all interactions $U>U_{c}$ and therefore realized. At the critical point $U_{c}$ indicated by a full arrow shows the transition from this Mott-Heisenberg insulator to a paramagnetic metal.

$m$ when approaching the transition therefore allows to distinguish a first-order transition from a continuous one.

Ground-state energy. Figure 4.22 shows the ground-state energy $E_{0}$ of three different phases as a function of Coulomb repulsion $U / t$ at the isotropic point $t_{\perp} / t=1.0$. The transition from a paramagnetic insulator to a paramagnetic metal, which has been discussed in the previous section, is indicated by a dashed arrow for comparison. However, by including the antiferromagnetic Weiss field on the bath sites, an additional solution with even lower energy exists for large interaction strength. For all interaction strengths, its energy is lower than the one of the paramagnetic insulator (data points connected by a dashed line in the figure), which is not realized anymore in the phase diagram. The new solution amounts to an antiferromagnetic insulator and reaches down to intermediate coupling strengths such that its energy crosses with the one of the paramagnetic metal. This transition indicated by an arrow in figure 4.22 is investigated in this section.

At large interchain coupling, the antiferromagnetic solution exists also for interaction strengths $U<U_{c}$. This means that the self-energy functional shows two energetically degenerate stationary points at the transition that differ considerably in the values of the variational parameters. Therefore, considering only the energy already indicates the discontinuous nature of the Mott transition at large $t_{\perp} / t$.

However, when reducing $t_{\perp}$, the region $U<U_{c}$, where two different stationary points can be clearly identified, shrinks. At small interchain hopping strengths, no coexistence region can be observed and the energy of the antiferromagnet seems to smoothly approach 

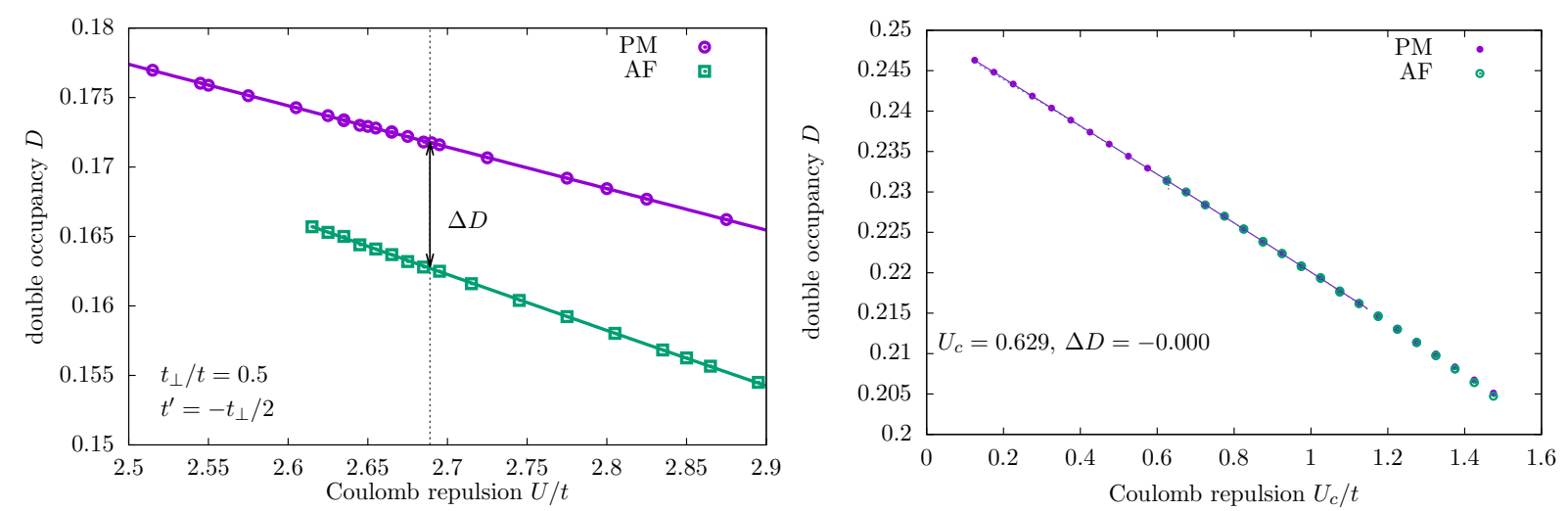

Figure 4.23: Jump in double occupancy $D$ as a function of interaction strength $U$ for frustration $t^{\prime}=-t_{\perp} / 2$. In the left panel $t_{\perp} / t=0.5$ and the double occupancy jumps at the transition $U_{c}$. For interaction strengths $U \lesssim U_{c}$ the antiferromagnetic insulator still exists. The right panel shows $t_{\perp} / t=0.05$ where neither a jump in $D$, nor a coexistence region is visible.

the one of the paramagnetic metal so that a clear kink can not be identified at $U_{c}$. This necessitates the investigation of double occupancy $D$ and staggered magnetization $m$ close to the transition.

Double occupancy. In case of the paramagnet, the double occupancy $D=\partial \Omega / \partial U$ allows to identify a discontinuous Mott transition via a jump at $U_{c}$ and suggests to investigate the transition from antiferromagnetic insulator to paramagnetic metal, too. Compared to the paramagnetic transition, the jump in double occupancy is much smaller at the transition studied here. This can be seen in figure 4.22 when comparing the slopes of the energies for the three phases close to the two different transitions. Figure 4.23 shows the double occupancy in close vicinity of the transition for two different hopping strengths.

At intermediate $t_{\perp} / t=0.5$, a clear jump in double occupancy can be identified at $U_{c}$. In the quasi-one-dimensional case $t_{\perp} / t=0.05$ (right panel), the double occupancy of both solutions nearly coincides already for $U>U_{c}$, and right at the transition $U_{c}$ the difference of $D$ is within the error bars of the calculation of $D$ as a numerical derivative.

The results of this analysis are summarized in figure 4.24, which shows the jump in $D$ at the transition for the two different values of relative frustration used. In both cases, the jump size seems to be proportional to $t_{\perp} / t$ for intermediate interchain coupling. However, for small interchain hopping $t_{\perp} / t \sim 0.4$, the jump size decays much slower leading to an extended tail region down to $t_{\perp} / t \sim 0.2$. For even smaller $t_{\perp} / t$, the double occupancy suggests a continuous Mott transition.

Staggered magnetization. In contrast to the double occupancy, which has to be calculated as a numerical derivative of the grand potential, the staggered magnetization can be accessed directly from the lattice Green function. As the metallic phase is paramagnetic, the staggered magnetization of the antiferromagnetic insulator at the transition $U_{c}$ corresponds also to the jump in magnetization. In addition, the jump in double occupancy turned out to be very small even at intermediate hopping strengths. It turns out that the staggered magnetization has a comparably large value and is therefore better suited to 


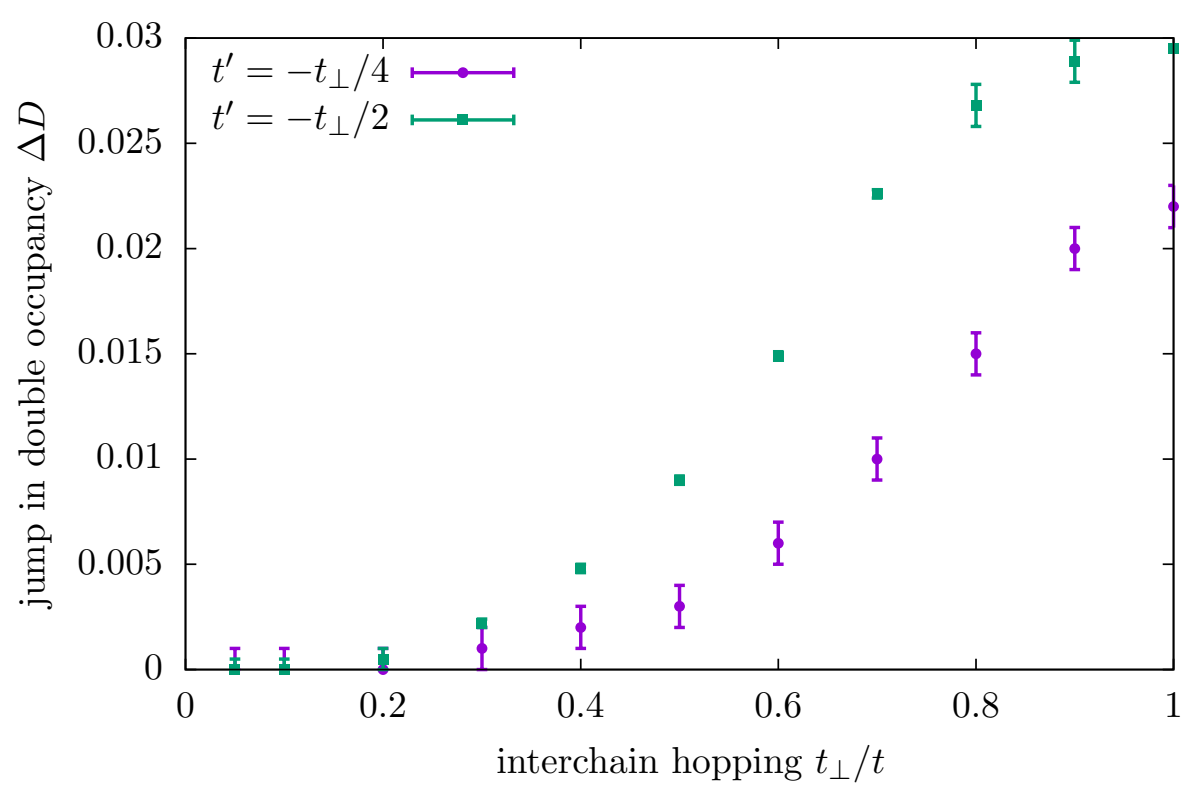

Figure 4.24: Jump in double occupancy $D$ at the transition $U_{c}$ as a function of interchain hopping $t_{\perp}$. Results are shown for two different relative frustration strengths.

investigate a putative continuous transition at small $t_{\perp} / t$.

Figure 4.25 shows the jump in magnetization at $U_{c}$ for both frustration values. Just like the jump in double occupancy, $\Delta m$ also shows a nearly linear increase with $t_{\perp} / t$ for intermediate interchain hopping. In contrast to $\Delta D$, the magnetization allows to identify a finite jump size even at $t_{\perp} / t=0.2$. The trend of a slower decay of the jump size at small $t_{\perp} / t$ is also found in the staggered magnetization and asks for an investigation of larger clusters to analyze the influence of finite-size effects on this region. Especially for the smaller relative frustration of $t^{\prime}=-t_{\perp} / 4$, an exponential decay of $\Delta m$ in the limit $t_{\perp} \rightarrow 0$ is not excluded.

At first sight, it might seem odd that the jump size in $m$ is larger for the stronger frustrated system, as frustration is found to suppress antiferromagnetic order at sufficiently strong frustration. However, figure 4.25 shows the staggered magnetization of the antiferromagnetic insulator at the transition $U_{c}$. As can be seen in the left panel of figure 4.21, the transition lines differ significantly for the two values of relative frustration, especially at intermediate and large $t_{\perp} / t$. The higher critical interaction strength for $t^{\prime}=-t_{\perp} / 2$ and the first-order transition in this region causes a transition from a paramagnetic metal to an insulator with pronounced antiferromagnetic order. When comparing with the much smaller critical interaction strength $U_{c}$ for the lower frustrated case, it is not too surprising that the staggered magnetization is larger at the transition. 


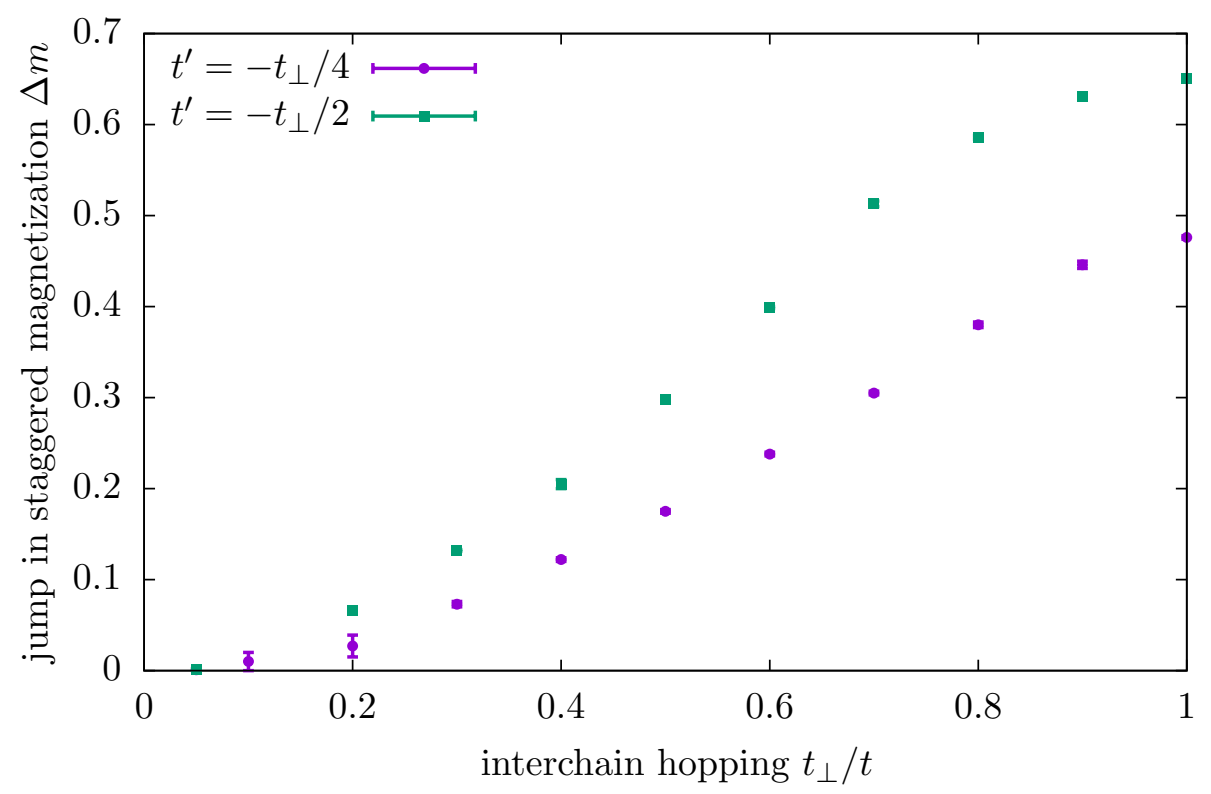

Figure 4.25: Jump in staggered magnetization $m$ at the transition $U_{c}$ as a function of interchain hopping $t_{\perp}$. Results are shown for two different relative frustration strengths.

\section{\begin{tabular}{l|l} 
Summary & 4.4
\end{tabular}}

The results of this chapter can be related to recent experiments on organic conductors with a half-filled band, where putative quantum critical behavior in the crossover region is discussed $\left[\mathrm{FMT}^{+} 15, \mathrm{AJKW}^{+} 15\right]$. Both $\kappa$ - $(\mathrm{BEDT}-\mathrm{TTF})_{2} \mathrm{Cu}_{2}(\mathrm{CN})_{3}$ and $\mathrm{EtMe}_{3} \mathrm{Sb}\left[\mathrm{Pd}(\mathrm{dmit})_{2}\right]_{2}$ are thought to be layered systems, with Hückel parameters close to an equilateral triangular lattice [KK11]. For the latter compound, careful ab-initio calculations [NYI12] instead show an appreciable 1D anisotropy with a ratio of interchain to intrachain transfer around 0.82 .

This asymmetry is taken into consideration in the anisotropic Hubbard model investigated in this thesis. The combination of VCA at $T=0$ and CDMFT at finite $T$ gives strong evidence for Mott quantum criticality in coupled Hubbard chains at half-filling. By using the interchain hopping $t_{\perp}$ as a control parameter, the second-order critical end point $T_{c}$ of the interaction-driven MIT could be tuned down to zero in the presence of strong anisotropy. Above a threshold value of $t_{\perp}^{c} / t \simeq 0.2$, the transition is found to be discontinuous, which shows in a jump in the double occupancy and at large interchain coupling the self-energy functional even has two distinct minima that indicate a pronounced coexistence region. In the nearly isotropic case, the Fermi-surface topology is the one of a conventional metal, but for $t_{\perp} / t \lesssim 0.7$ it changes to a Fermi surface with electron and hole pockets. At intermediate $t_{\perp}$, the volume of the pockets shrinks when approaching the MIT and is cut off at $U_{c}$ by the first-order transition. This is in contrast to the strongly anisotropic case $t_{\perp} / t \lesssim 0.2$. There, the volume of the Fermi pockets continuously vanishes at the transition and neither a jump in the double occupancy nor a coexistence region in the SEF is visible. 
In the crossover region at high temperature $T \gg T_{c}$, the scaling behavior of resistivity curves is usually attributed to hidden 2D Mott quantum criticality [TVTD11, VTRD15]. Future studies on the continuous Mott transition for $t_{\perp} / t \lesssim 0.2$ at zero temperature could help to gain further insights into this issue. As a similar breakup of the Fermi surface into pockets was found in coupled spinless fermionic chains [BGBG06], another interesting question is whether quantum critical behavior also emerges in a spinless system.

Both in the VCA and the CDMFT studies, a $2 \times 2$ plaquette cluster was used, which is known to overestimate the singlet formation [MJPH05]. As former CDMFT studies on the same model using larger clusters of up to 16 sites provided evidence for a continuous dimensional-crossover-driven MIT down to the lowest accessible temperatures [RA12], the quantum critical behavior for $t_{\perp} / t \lesssim 0.2$ is expected to be robust nevertheless. When reducing the range of antiferromagnetic spin fluctuations, this scenario should not be restricted to quantum cluster descriptions of the system but also emerge in lattice simulations. This could be realized by geometrical frustration such as a stronger diagonal hopping [KT06, NSST08, YSEO13] or by disorder [BHV09, $\mathrm{FMI}^{+}$15]. However, when introducing frustration a severe sign problem renders lattice QMC simulations very expensive [RAP15]. In this respect, a promising route is offered by tensor network methods [Orú14] adapted recently to fermionic systems [CV09, CEVV10, Cor16].

A first step into this direction consists in comparing the VCA results at zero temperature to density-matrix renormalization group (DMRG) [Whi92, Sch05, Sch11] studies on Hubbard ladders with the same hopping matrix as the model studied here. Albeit being mainly used for the investigation of one-dimensional models, DMRG has been applied successfully to two-dimensional models in recent years, too [YHW11, DMS12, SW12]. This route is pursued in collaboration with Georg Ehlers and Reinhard Noack, who developed an efficient hybrid $k$-space formulation of DMRG that allows the treatment of comparably large lattice sizes [ESLN15].

Another way of approaching the problem with lattice simulations is to use variational Monte Carlo techniques (VMC). In collaboration with Marcin Raczkowski, Fakher Assaad and the group of Masatoshi Imada, VCA, CDMFT, and VMC are compared in order to approach the question of putative Mott criticality in the antiferromagnetic phase. A quantum critical line as observed in the paramagnetic case would allow for the study of a marginal quantum critical point (MQCP) [Ima04]. As Hartree Fock calculations for such a MQCP lead to unconventional exponents in agreement with those obtained from experiments, an investigation of a MQCP with techniques beyond Hartree Fock is desirable. In order to clarify the influence of finite-size effects on the behavior of the MIT in the antiferromagnetic phase for small interchain hopping strengths, the investigation of larger clusters within VCA is needed. This might even necessitate a combination of VCA with other efficient cluster solvers that can be used at zero temperature, e.g. DMRG.

The results motivate adding the anisotropy $t_{\perp}$ as a new axis in the phase diagram, along which the critical end point $T_{c}$ can be tuned to zero. It still has to be verified in future studies whether the quantum critical behavior can explain the unconventional Mott criticality that has been observed recently in layered organic conductors. The findings for the paramagnetic case and the preliminary results for the antiferromagnetic phase encourage future studies along this line. 


\section{\begin{tabular}{r|r} 
Unconventional Phases in the Kondo & \\
Lattice Model & 5
\end{tabular}}

In this chapter, different unconventional phases of the Kondo lattice model are investigated by variational cluster approximation. A publication containing the VCA results of this chapter in collaboration with Salvatore R. Manmana and Riccardo Gezzi, who provided calculations on superconductivity using the equation of motion technique, is in preparation and about to be finalized. The chapter is structured as follows.

First, section 5.1 shows results that are obtained within variational cluster approximation for the paramagnetic phase. A focus is set on Kondo singlet formation at strong coupling. In the second section (5.2) the antiferromagnetic solution at weak to intermediate coupling strengths is investigated. The solution is analyzed with respect to its Fermi surface topology and results are compared to those of other numerical methods.

Section 5.3 addresses the question of whether s-wave pairing is present in the Kondo lattice model. A seemingly superconducting solution is identified to be a mean-field-like artifact and no robust s-wave superconductivity is found.

Finally, in section 5.4 the model is probed for d-wave superconductivity. A stable solution is identified over a broad parameter range and its interplay with antiferromagnetism at small interaction strengths is analyzed.

Section 5.5 summarizes the results of this chapter.

\section{\begin{tabular}{r|r} 
The Paramagnetic Phase of the Kondo Lattice & Model \\
M.1
\end{tabular}}

In this section, the paramagnetic phase of the Kondo lattice model is investigated using the variational cluster approximation.

As VCA does not allow for phases with broken symmetries unless proper Weiss fields are added, it is possible to investigate the paramagnetic solution at all coupling strengths irrespective of the "true" ground state of the system. That means that although the system might be antiferromagnetic at weak coupling, without adding a Weiss field the stationary point of the self-energy functional will always correspond to a paramagnetic solution.

As will be seen later, this phase is the correct/physical solution at large coupling close to and at half-filling. However, even for other parameter regimes, where the next sections will show that phases with broken symmetries are realized, the paramagnetic solution serves as a starting point and always has to be considered as a reference. In the end, even if additional solutions with broken symmetry such as antiferromagnetic or superconducting order are found, it has to be checked, whether their energy is lower than the "trivial" solution without broken symmetry.

The section is structured as follows. In the first subsection the influence of including different sets of intra-cluster hopping terms into the set of variational parameters on the stationary point of the self-energy functional (SEF) is scrutinized. Especially for the $3 \times 2$ cluster that is excessively used in subsequent calculations investigating the effect of using anisotropic hopping inside the cluster is important. The second subsection shows results 
for the Kondo insulator at half-filling. In the large coupling region where the solution corresponding to this stationary point turns out to be the physical one, these results are compared with other state-of-the-art numerical techniques. Finally, the paramagnetic solution in the doped region is analyzed and sets the basis for the discussion of the Fermi surface in section 5.2.2.

\section{Influence of the Intra-Cluster Hopping $\mid 5.1 .1$}

Especially for small clusters the influence of the variation of the intra-cluster hopping on resulting observables has to be checked before adding symmetry breaking Weiss fields. One would expect, that for small interactions $J / t \rightarrow 0$ the technique becomes exact with the approximated self-energy going to zero. When investigating the SEF it turns out that there is one stationary point where $t^{\prime}$ minimizes $\Omega$ and one where it maximizes the functional. For both solutions the energy is shown as a function of coupling strength $J / t$ in figure 5.1. The solution with $t^{\prime} \approx t$ in the large $J$ limit is more intuitive than the one with $t^{\prime}=0$ as the cluster decoupling of the system only slightly changes the intra-cluster hopping. Nevertheless, for small interactions $J / t<J_{c}^{t} / t=1.75$ the hopping increases rapidly, which can be explained with the fact, that the self-energy (and hence the parameter of the reference system) does not influence the observables much. Still, it is not plausible or intuitive, why such a huge intra-cluster hopping as $t^{\prime}=10 t$ should be a physically meaningful choice. Instead, one has to be careful as perhaps finite-size effects dominate in this region.

In case of the $2 \times 2$ cluster an inflection point is found at a value $J_{c}^{t} / t \approx 1.75$, which could be taken as a naive lower limit from which on the approximation is justified. For the larger clusters considered in figure 5.1 there is even a sharp kink in the intra-cluster hopping when it comes to small couplings $J_{c}^{t} / t \lesssim 0.84$. Again, for larger $J / t$ the small deviation of $t^{\prime}$ from $t$ seems to be reasonable, but the steep increase for small $J / t$ after the kink has to be explained.

As will be shown in the next section, the quasiparticle gap vanishes for weak coupling $J<J_{c}^{t}$ and the paramagnetic system becomes metallic. This transition not only explains the kink in the value of $t^{\prime}$, but also the large values of $t^{\prime}$ for small $J$ could be explained by considering a Fermi liquid picture, where electronic fluctuations inside the clusters are enhanced. It is furthermore quite remarkable how close the free energies per site are for $3 \times 2$ and $4 \times 2$ clusters.

Looking at the corresponding energies of both solutions, one notes that the solution with $t^{\prime} \approx 1$ is always (slightly) lower and should therefore be preferred. For small interactions the energies approach each other as expected: In the limit $J / t \ll 1 \mathrm{VCA}$ becomes exact as the self-energy goes to zero. Both solutions should, in this limit, give the same result. In the limit of large interactions the energies of the solutions are close to each other. This makes sense, as one expects to see the singlet insulator, where the hopping is suppressed and does not influence the resulting observables too much. Indeed, when calculating the spin-spin correlation $\left\langle s^{c} S^{f}\right\rangle=\partial F / \partial J$ per site in the limit of large $J / t$, the value approaches $\left\langle s^{c} S^{f}\right\rangle=-3 / 4$, which is expected in the case of singlet formation.

Using clusters that are not as symmetric as the $2 \times 2$ plaquette also poses the question of how to deal with this anisotropy when varying the intra-cluster hopping. Varying all hopping parameters inside the cluster separately of course results in the same optimized 

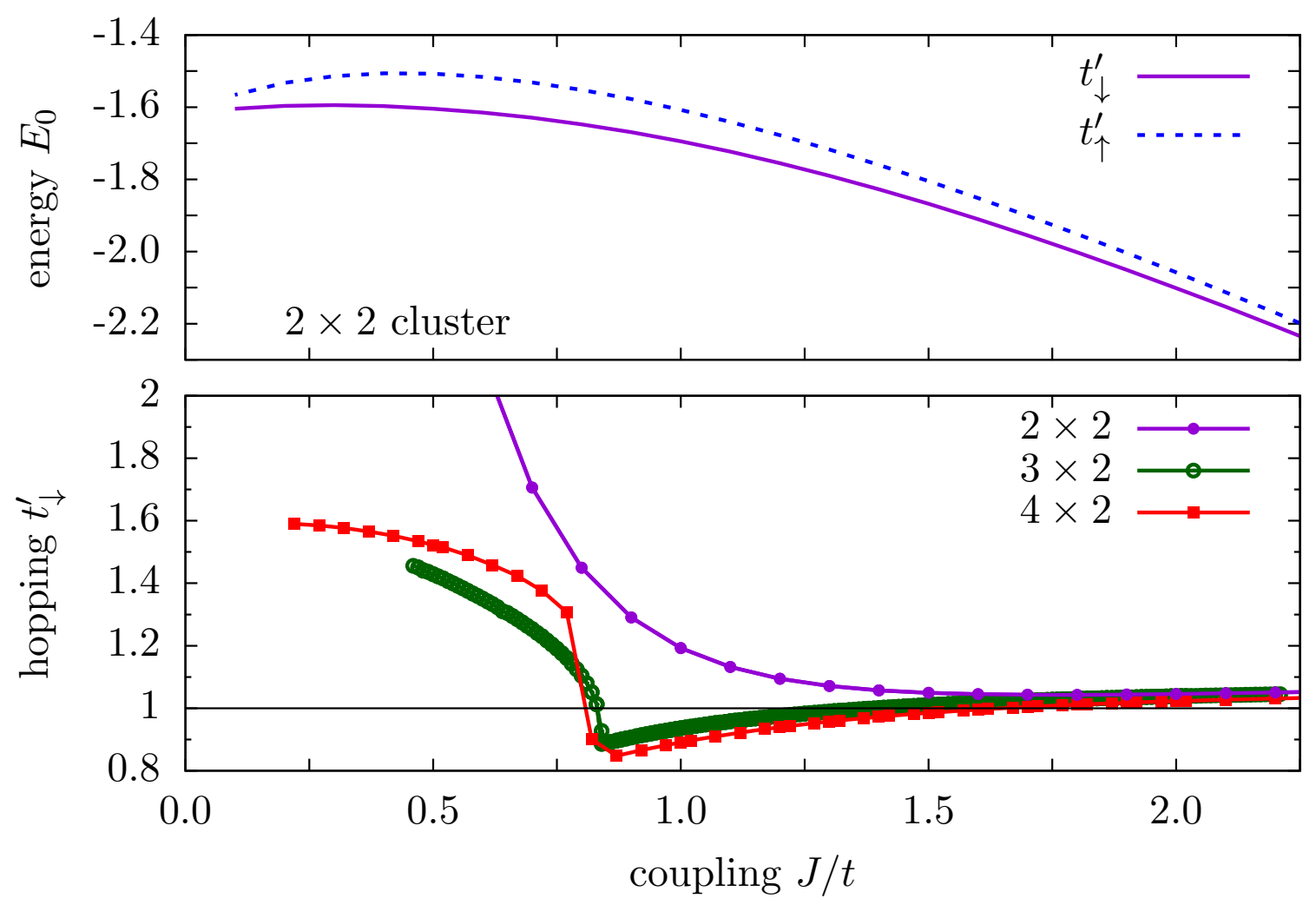

Figure 5.1: In the top panel, the energy for both stationary points of the SEF are shown: The SEF has a maximum with respect to the cluster hopping at $t_{\uparrow}^{\prime}=0$ and a minimum at $t_{\downarrow}^{\prime} \neq 0$. In the bottom panel the intra-cluster hopping for $2 \times 2,3 \times 2$ and $4 \times 2$ clusters is plotted as a function of $J / t$. Only the solution with $t_{\downarrow}^{\prime}$ is shown.

hopping values for hopping terms that are connected with a space symmetry. Considering the $3 \times 2$ cluster this means, that one is still left with three hopping parameters that in principle should be varied separately, see Fig. 5.2.

The anomaly in the intra-cluster hoppings remains, especially the vertical hopping strength inside the cluster changes drastically for small interaction strengths. In the end, one has to check the behavior of the different $t^{\prime}$ values for small interactions in the antiferromagnetic solution to see whether this anomaly is due to the finite size of the cluster or due to the (artificial and unphysical) choice of the paramagnet in this region. This will be done in section 5.2.3, where the question of the influence of $t^{\prime}$ on the quality of the results is readdressed.

\section{The Kondo Insulator at Half-Filling}

In the Kondo lattice model the simplest kind of insulator is the (atomic) Kondo insulator at half-filling that consists of local singlets between f-spins and conduction electrons. Here, we continue the investigation of the paramagnetic solution (see section 5.1.1) by doping the system away from half-filling to determine the spectral gap $\Delta \mu=\Delta_{q p} / 2$. One characteristic of an insulator is a vanishing electronic compressibility $\kappa=-\frac{\partial n}{\partial \mu}, n$ being the charge carrier density and $\mu$ the chemical potential. This corresponds to a plateau at half-filling in a $n$-vs.- $\mu$ plot, which we investigate as a function of $J / t$ and cluster size for 


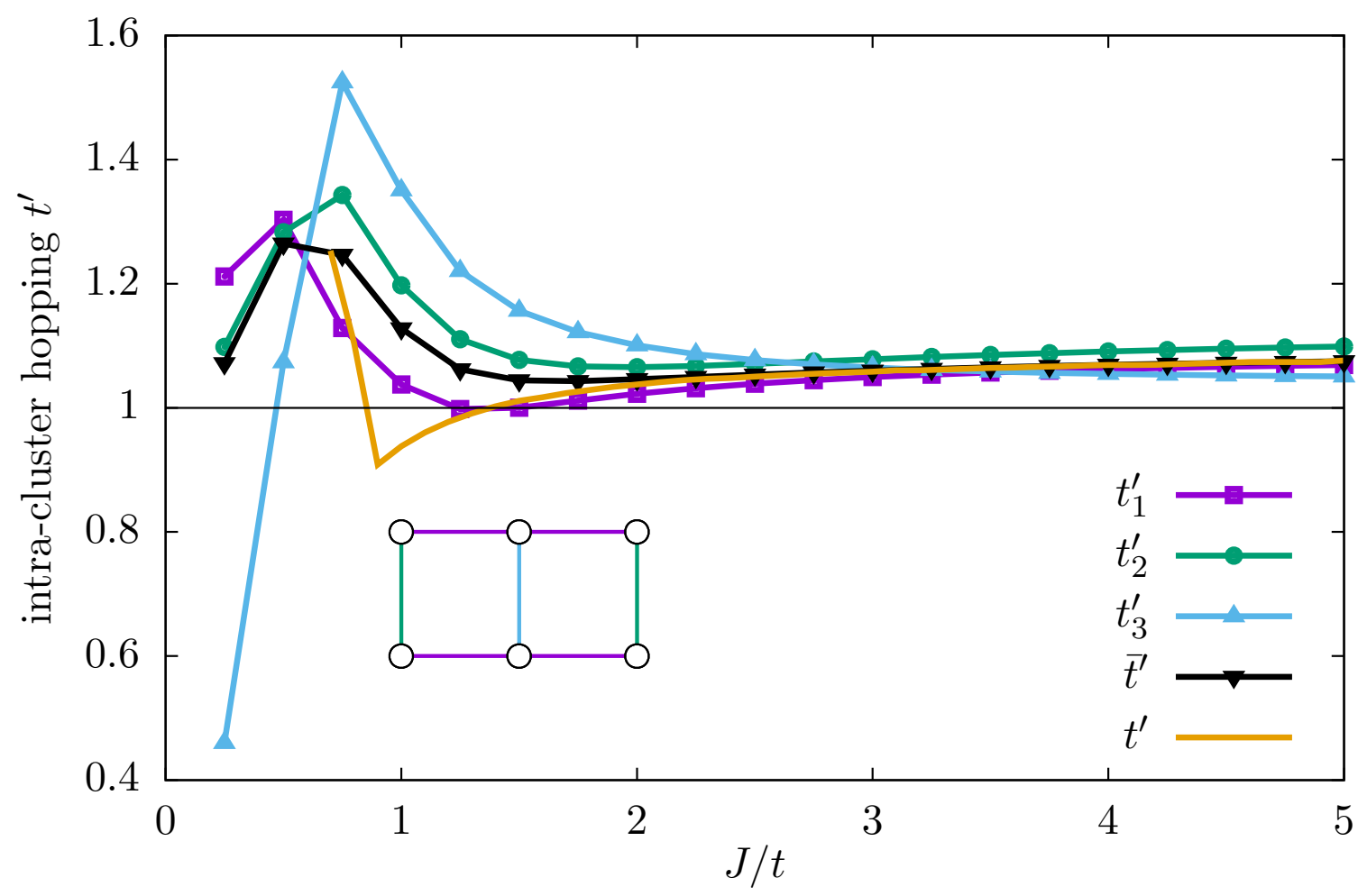

Figure 5.2: Optimized intra-cluster hopping strengths inside a $3 \times 2$ cluster as opposed to a single hopping parameter varied for the same cluster at half-filling in the paramagnetic solution. The mean value $\bar{t}^{\prime}=\left(4 \cdot t_{1}^{\prime}+2 \cdot t_{2}^{\prime}+t_{3}^{\prime}\right) / 7$ is plotted for comparison.

the paramagnetic solution. Details of the calculations off half-filling are given in the next subsection. Here, we focus only on the consequences for the system at half-filling such as the just mentioned plateau. Based on the exact, finite-size extrapolated QMC results of Assaad [Ass99] one expects to find a finite quasiparticle gap for all positive (that is antiferromagnetic) values of $J / t$ if considering the correct ground state for every $J / t$. This is an important point as only for $J>J_{c}$ we expect the paramagnetic solution to be the ground state, below $J_{c}$ the system orders antiferromagnetically and the paramagnet is not the ground state solution at $T=0$. The antiferromagnet will be discussed in the next section.

Instead of calculating the electron filling as a function of the chemical potential, it is possible to obtain the quasiparticle gap by doping the system a bit away from half-filling. Using $\Delta_{\mathrm{qp}}=\lim _{\epsilon \rightarrow 0^{+}} \mu(n=1+\epsilon)$ it is possible to extract the quasiparticle gap more efficiently.

To do so, one Legendre transforms the self-energy functional with respect to the electron density $n$ and adds the chemical potential $\mu$ to the set of variational parameters. Thereby, it is possible to set the electron filling to a certain value and determine the chemical potential via the stationarity principle. In order to determine the quasiparticle gap one then calculates the stationary points at $n=1+\epsilon$ as a function of $J / t$.

The choice of a finite $\epsilon$ leads to an error in $\Delta_{\mathrm{qp}}$, but for fillings close to half-filling (e.g. for $n=1.001)$ the system is metallic and the chemical potential nearly coincides with the quasiparticle gap $\Delta_{\text {qp }}$ at half-filling. For instance, in figure 5.3 the plateau in the $n$-vs.- $\mu$ curve ends at $\mu= \pm \Delta_{\mathrm{qp}}$, but choosing $\mu(n=1.001)$ results in an error of $\Delta\left(\Delta_{\mathrm{qp}}\right)<0.01$. 


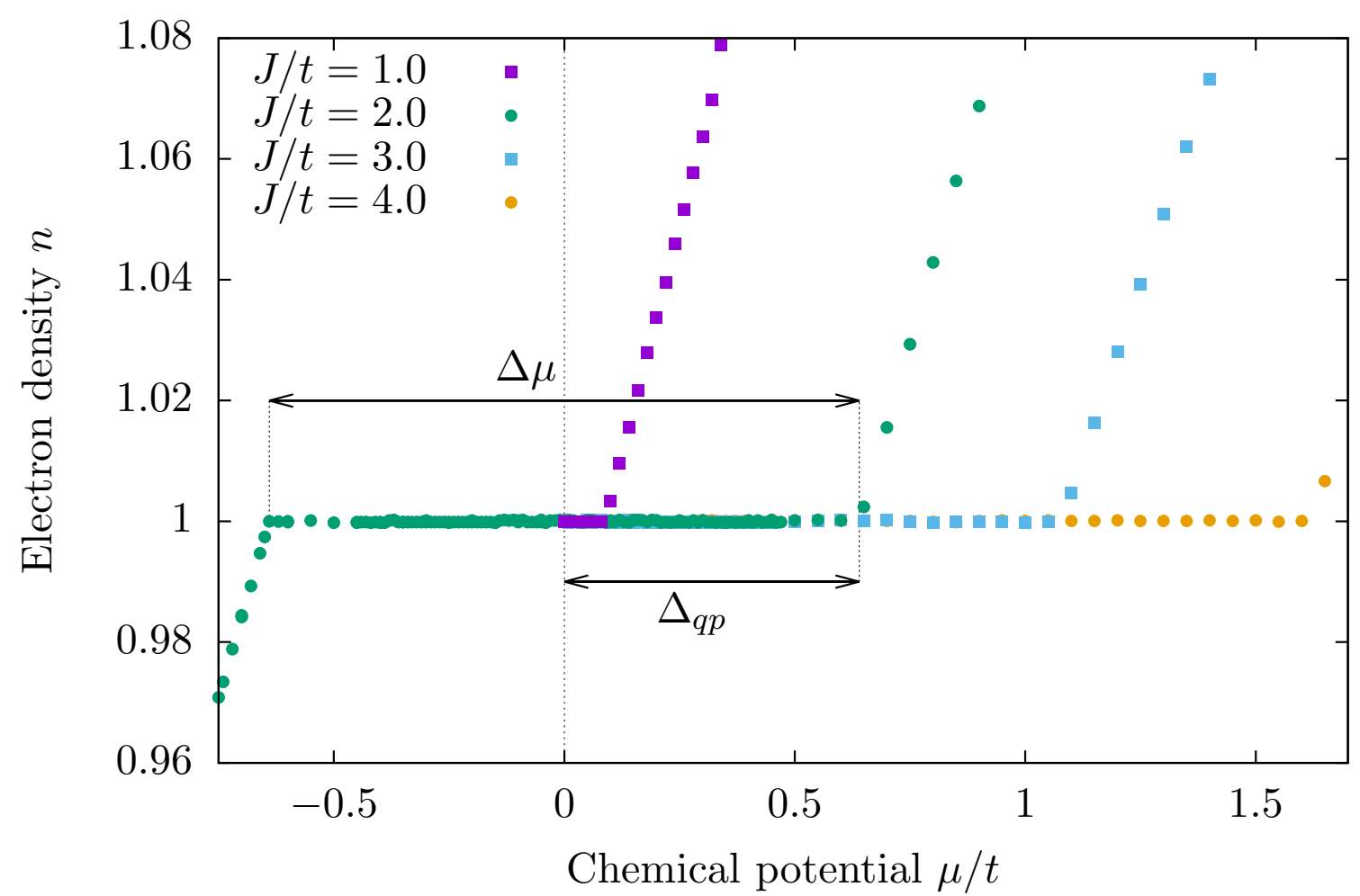

Figure 5.3: Plateau in the $n$-vs.- $\mu$ plot at half-filling using a $3 \times 2$ cluster with variation parameters $t^{\prime}$ and $\mu^{\prime}$.

Figure 5.4 shows the quasiparticle gap as a function of $J / t$ for $2 \times 2$ and $3 \times 2$ cluster, $\mu(n=1.001)_{3 \times 2}$ is shown for comparison. As expected the system is a paramagnetic insulator for large interactions and down to intermediate $J$ a finite gap is observed. The gap depends only little on the cluster size for large and intermediate $J$, but one has to note that the $2 \times 2$ cluster gives deviating results for interactions $J / t \lesssim 1.8$, which is close to the minimum of $t^{\prime}$ that we encountered already in section 5.1.1, figure 5.1. Down to $J / t \approx 1.6$ the gap of the $3 \times 2$ cluster can be fitted nicely with a second order polynomial, but for smaller $J$ it deviates, too. Comparing the quasiparticle gap to exact quantum Monte Carlo (QMC) data of reference [Ass99], it shows good agreement down to $J_{c} / t_{\mathrm{QMC}}=1.45$, where the ground state becomes antiferromagnetic. However, as we should see in the next subsection, the critical interaction strength $J_{c} / t$ within VCA using these clusters is larger than 1.45 and for a comparison one should consider the VCA gaps of the antiferromagnetic solution for $J<J_{c}$. Due to the different - finite-size affected critical $J_{c}$ values one would also have to extrapolate the VCA results in order to compare the antiferromagnetic gaps.

In case of the $3 \times 2$ cluster, the paramagnetic gap reduces for even smaller values of $J / t$ and finally vanishes at $J / t \approx 0.84$. This value coincides with the anomaly in $t^{\prime}$, which is shown in figure 5.2. The transition from the paramagnetic insulator to a paramagnetic metal for $J / t<0.84$ quite naturally explains the kink in the cluster hopping and shows that the proper treatment of antiferromagnetism is needed for small $J$. That means that within VCA the minimal coupling strength $J_{\mathrm{KI}}$ which is needed to form the (Kondo) insulator is finite. 


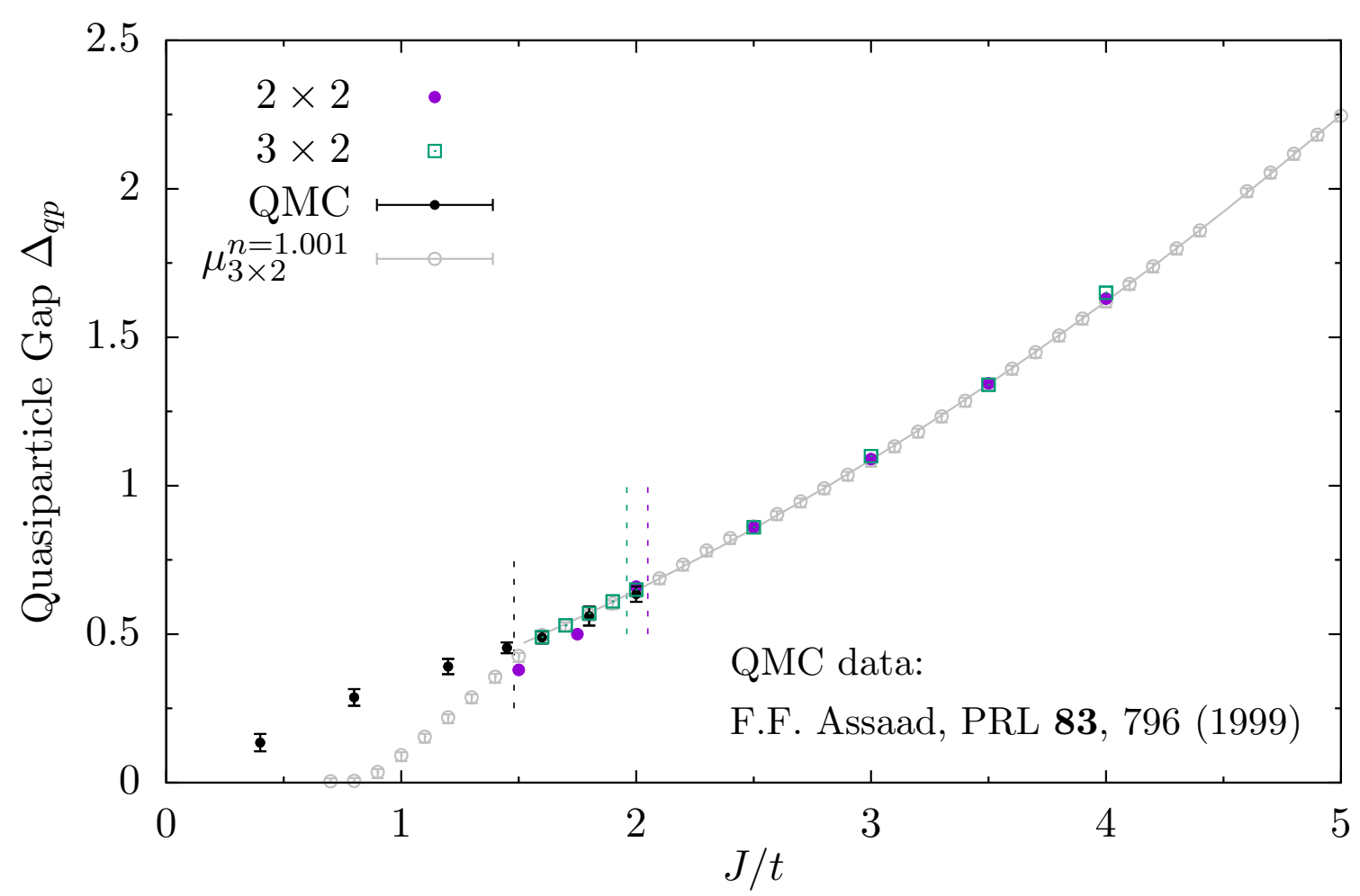

Figure 5.4: Quasiparticle gap of the paramagnetic solution at half-filling using the $2 \times 2$ and $3 \times 2$ cluster with variational parameters $t^{\prime}, \mu^{\prime}$. At the dashed lines the system changes from paramagnetic $\left(J>J_{c}\right)$ to antiferromagnetic, thus the paramagnetic solution for $J<J_{c}$ is not realized, which will be discussed in the next subsection. The circles denote the chemical potential $\mu(n=1.001)$ using the $3 \times 2$ cluster, the solid line is a fitted parabola and black dots indicate exact QMC results by Assaad taken from reference [Ass99].

\section{Doping the Paramagnet $\mid 5.1 .3$}

To dope the system away from half-filling $(n \neq 1)$ one needs to include $\mu^{\prime}$ as a variational parameter [AAPH06a]:

$$
\mathcal{H}_{\mu^{\prime}}=\mu^{\prime} \sum_{\mathbf{R}} n_{\mathbf{R}}
$$

Only then thermodynamical consistency for the estimate of the electron density $n$ is ensured, namely:

$$
\langle n\rangle=-\frac{\partial \Omega}{\partial \mu} \stackrel{\mu^{\prime}=\mu_{\mathrm{opt}}^{\prime}}{=} \frac{1}{N} \int_{\mathcal{C}<} \frac{d z}{2 \pi i} \operatorname{Tr}\left[\delta_{R R^{\prime}} \delta_{\sigma \sigma^{\prime}} \mathbf{G}_{R \sigma, R^{\prime} \sigma^{\prime}}(\mathbf{k}, z)\right]
$$

It was shown in the previous section that for strong coupling $J$ the ground state of the Kondo lattice model at half-filling is paramagnetic. To be more precise, it consists of singlets between the local spins and the conduction band electrons. At large $J$ all electrons are bound into such singlets and the ground state is hence insulating.

This changes once the system is doped away from half-filling, as Kondo singlets are broken up and electrons can again move through the system. The result is a metal which naturally displays a Fermi surface, e.g. figure 5.5 shows the Fermi surface at $J / t=3.0$ and an electron filling of $n=0.90$. Despite the low electron filling the Fermi surface is large and 

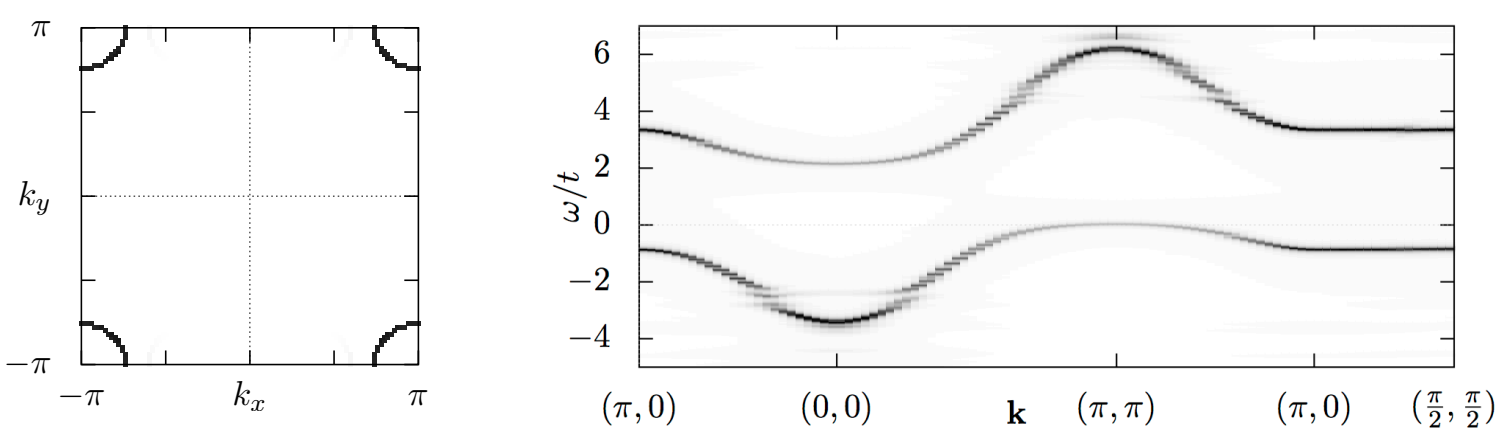

Figure 5.5: Left panel: Large Fermi surface deep in the paramagnetic phase $(J / t=3.5)$ away from half-filling $(n=0.9)$. Right panel: Corresponding spectral function $A(\mathbf{k}, \omega)$ along a certain path in the Brillouin zone. Dashed lines only indicate the zeros; the data shown was obtained with a $3 \times 2$ cluster.

measuring its area gives a value of $n_{\mathrm{FS}} \approx 1.9$. As already mentioned in section 1.3 .2 not only conduction electrons, but also f-spins participate in the charge transfer process for strong coupling. The reason is that conduction electrons can move through the lattice and form local Kondo singlets with the f-spins. Although only the electrons are mobile, effectively this can be viewed as moving Kondo singlets. Hence, the Fermi surface is made up of both electrons and f-spins, which means that $n_{\mathrm{FS}}=n_{\mathrm{el}}+n_{\mathrm{f}}=n+1$. For $\mathrm{J} / t=3$ the value of $n_{\mathrm{FS}} \approx 1.9$ suggests that one is already in the region of a (nearly perfect) heavy Fermi liquid with a large Fermi surface.

In the strong coupling regime, two resonances in the density of states are identified, see figure 5.6. For $J / t=8$ and $n=0.95$ they are separated by a value of $1.475 J$. This agrees with the value of $3 / 2 J$ which is expected in the limit $J / t \gg 1$ with minimal doping, see the discussion of the Kondo insulator in section 2.3.

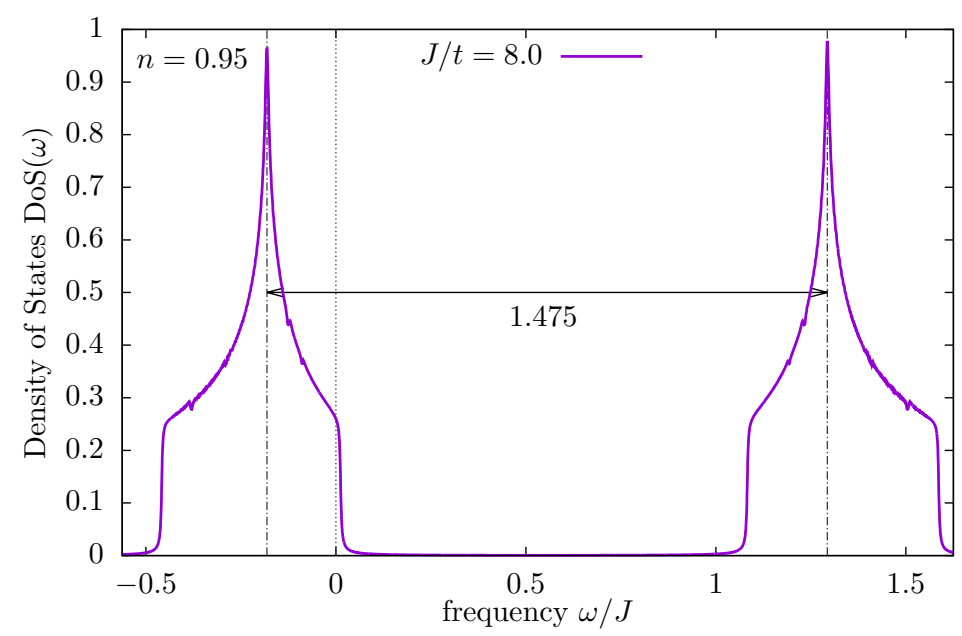

Figure 5.6: Density of states as a function of frequency $\omega$ for strong coupling $J / t=8$ close to half-filling $n=0.95$ using a $3 \times 2$ cluster. The two distinct resonances are separated by $1.475 J$. Doping a perfect Kondo insulator would lead to a value of $3 / 2 J$. 


\section{Magnetic Phase Diagram of the Kondo Lattice Model}

The competition between Kondo singlet formation and RKKY interaction sets the stage for Doniach's phase diagram [Don77]. One possibility to study this competition is the investigation of antiferromagnetic correlations, as RKKY interaction mediates an antiferromagnetic ordering of the f-spins and conduction electrons respectively. At half-filling, these antiferromagnetic correlations are expected to be suppressed from a certain $J_{c} / t$ on, as at this point Kondo singlet formation is energetically favorable and removes free electrons which could mediate the antiferromagnetic ordering.

When reducing the electron filling drastically, the model is expected to order ferromagnetically [PP07]. However, in this thesis only fillings close to half-filling will be considered such that this phase is not investigated.

To allow for the possibility of magnetic order on the cluster one has to add a fictitious Weiss field to the cluster Hamiltonian which takes the following form:

$$
\mathcal{H}_{\mathrm{AF}}=M \sum_{\mathbf{R}} e^{i \mathbf{Q} \cdot \mathbf{R}}\left(n_{\mathbf{R} \uparrow}-n_{\mathbf{R} \downarrow}\right) .
$$

In the case of Néel antiferromagnetism the wavevector is $\mathbf{Q}=(\pi, \pi)$. The strength $M$ of this field is then used as a variational parameter. Finally, the staggered magnetization of c-electrons can be obtained at the stationary point as

$$
m^{c}=\frac{1}{L} \int \frac{d z}{2 \pi i} \operatorname{Tr}\left[e^{i \mathbf{Q} \cdot \mathbf{R}}(-1)^{\sigma} G_{\mathbf{R} \sigma, \mathbf{R} \sigma}(z)\right]
$$

Unfortunately, the staggered magnetization of f-spins is only available on the cluster as the Green function of the system does only contain excitations with respect to the conduction electrons:

$$
m_{c l}^{f}=\frac{1}{L} \sum_{\mathbf{R}} e^{i \mathbf{Q} \cdot \mathbf{R}}\left\langle n_{\mathbf{R}, \uparrow}^{f}-n_{\mathbf{R}, \downarrow}^{f}\right\rangle .
$$

In the following, the antiferromagnetic solution of the Kondo lattice model is first of all investigated at half-filling. As it is possible to limit the minimal set of variational parameters to the strength of the antiferromagnetic Weiss field and the isotropic hopping strength of the cluster, even large clusters up to eight physical sites $(4 \times 2$ cluster $)$ are used. Therefore, a rough finite-size extrapolation of the critical coupling strength $J_{c}$ is evaluated and compared with exact QMC results. In the subsequent paragraph the ground state is further analyzed and a focus is set on the spin-spin correlator and the density of states. It is argued that inside the antiferromagnetic phase two distinct phases can be identified, which are separated roughly at the coupling which corresponds to maximal staggered magnetization.

The next step is then to leave the half-filled case in subsection 5.2.2, where the antiferromagnetic metal and finally a full phase diagram down to fillings of $n=0.8$ are investigated. Off half-filling, it is possible to identify two antiferromagnetic metals which correspond to the two regions that are found at half-filling. The evolution of the Fermi surface when tuning through the two antiferromagnetic and the adjacent paramagnetic metal as well as its implications to the Kondo singlet formation are discussed subsequently in paragraph 5.2 .2 . 
Finally, the study of section 5.1.1 is continued to the antiferromagnetic phase and the effect of choosing anisotropic hopping on the reference cluster both at and off half-filling is analyzed. Subsection 5.2.3 closes this chapter by showing that using an anisotropic cluster does not change the qualitative picture obtained with isotropic clusters.

\section{The Antiferromagnet at Half-Filling $\mid 5.2 .1$}

In the previous section the paramagnet was analyzed at half-filling and showed discrepancies from exact results for small and intermediate coupling strengths. This manifested in form of deviating quasiparticle gaps at intermediate couplings and even lead to metallic behavior for weak coupling in case of the $3 \times 2$ cluster.

When including an antiferromagnetic Weiss field, an antiferromagnetic phase is found for small coupling strengths. Here, the antiferromagnetic ordering and the perfect nesting of the corresponding Fermi surface leads to an antiferromagnetic gap, even at very small $J$. This insulator is therefore conceptually different from the paramagnetic insulator, which is formed due to spin singlet formation.

At some critical coupling strength $J_{c}$ the magnetization vanishes and the self-energy functional shows only one stationary point, namely the "trivial" one corresponding to the afore discussed paramagnetic insulator. For $J<J_{c}$ the system is antiferromagnetically ordered with a finite gap. Therefore, VCA correctly shows an insulator for all finite values of $J$.
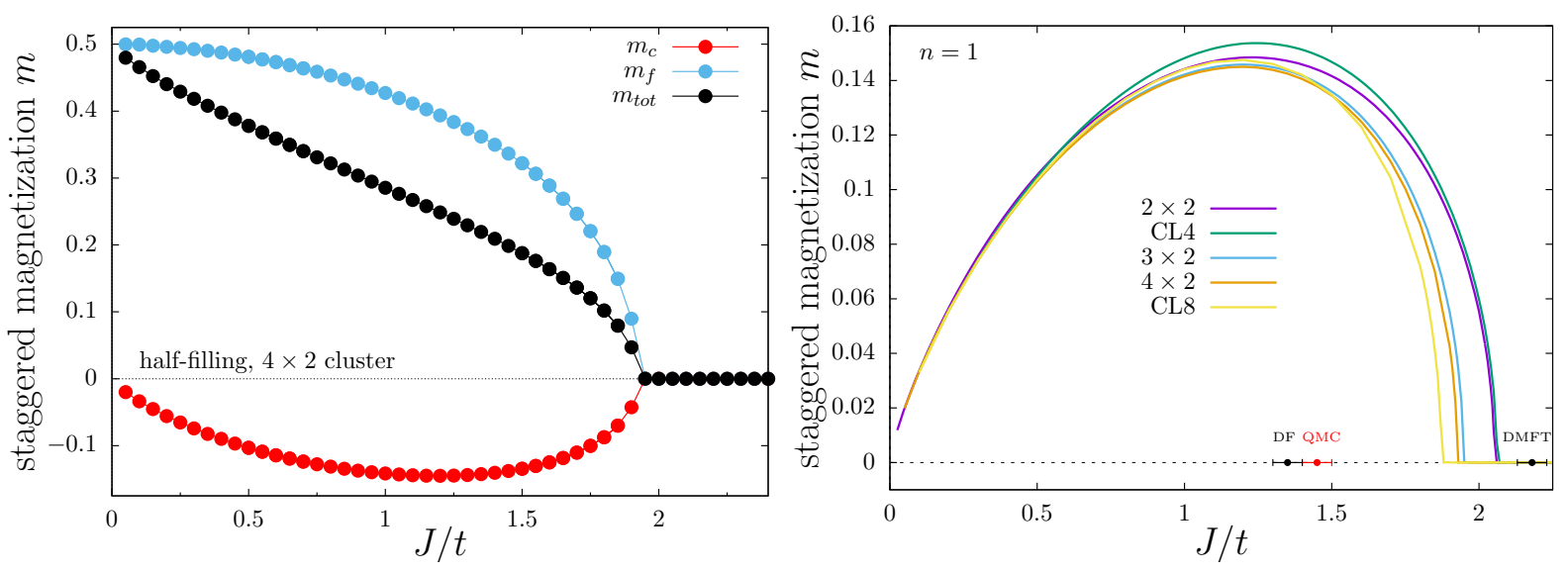

Figure 5.7: Left panel: Staggered magnetization of the conduction electrons $m_{c}$, the f-spins $m_{f}$ and the total staggered magnetization as a function of $J / t$ at half-filling using a $4 \times 2$ cluster. Right panel: Staggered magnetization $m$ for different cluster sizes and geometries, shown in figure 3.10. As a reference, values of $J_{c} / t$ obtained with Dual Fermions [Ots15], Quantum Monte Carlo [Ass99] and DMFT+NRG [Bod13] are shown.

Figure 5.7 shows in the left panel the staggered magnetization of the conduction band electrons $m$ and of the f-spins $m_{f}$, as well as the total magnetization $m^{\text {tot }}=m+m_{f}$ using a $4 \times 2$ cluster. However, it has to be noted that the staggered magnetization of the localized spins $m_{f}$ is calculated on the cluster only as the Green function contains only information of the conduction electrons. For this reason, the discussion will from here on focus on the staggered magnetization of the conduction band electrons $m$. In order to show the antiferromagnetic ordering between conduction electrons and f-spins, the staggered magnetization $m$ is plotted with a negative sign. As the choice of sign is 
somewhat arbitrary (the self-energy functional is symmetric with respect to the Weiss field strength $M_{c}$ ) subsequent plots will use positive values of the staggered magnetization $\mathrm{m}$. The strength of $m$ increases for small couplings with the coupling strength and reaches a maximum at $J_{\max }$. For larger $J$ it decreases rapidly and vanishes smoothly at the critical coupling $J_{c}$, where the system stays insulating, but without magnetic order.

Compared to the DMFT results of reference [Bod13], where a NRG solver was used to precisely capture the low energies inherent to Kondo (lattice) systems, the magnetization curve has the same characteristics, but the absolute values differ. In particular the critical coupling strength $J_{c}$ is lowered. This can be understood by recalling that the electronic fluctuations inside the $4 \times 2$ cluster are a natural antagonist of antiferromagnetic order. By increasing the size of the cluster, the spatial extend of the fluctuations which enter the reference self-energy grows and thereby changes the variational space for the determination of stationary points. Systematically increasing the cluster size can allow to determine the value of $J_{c}$ after a finite-size extrapolation.

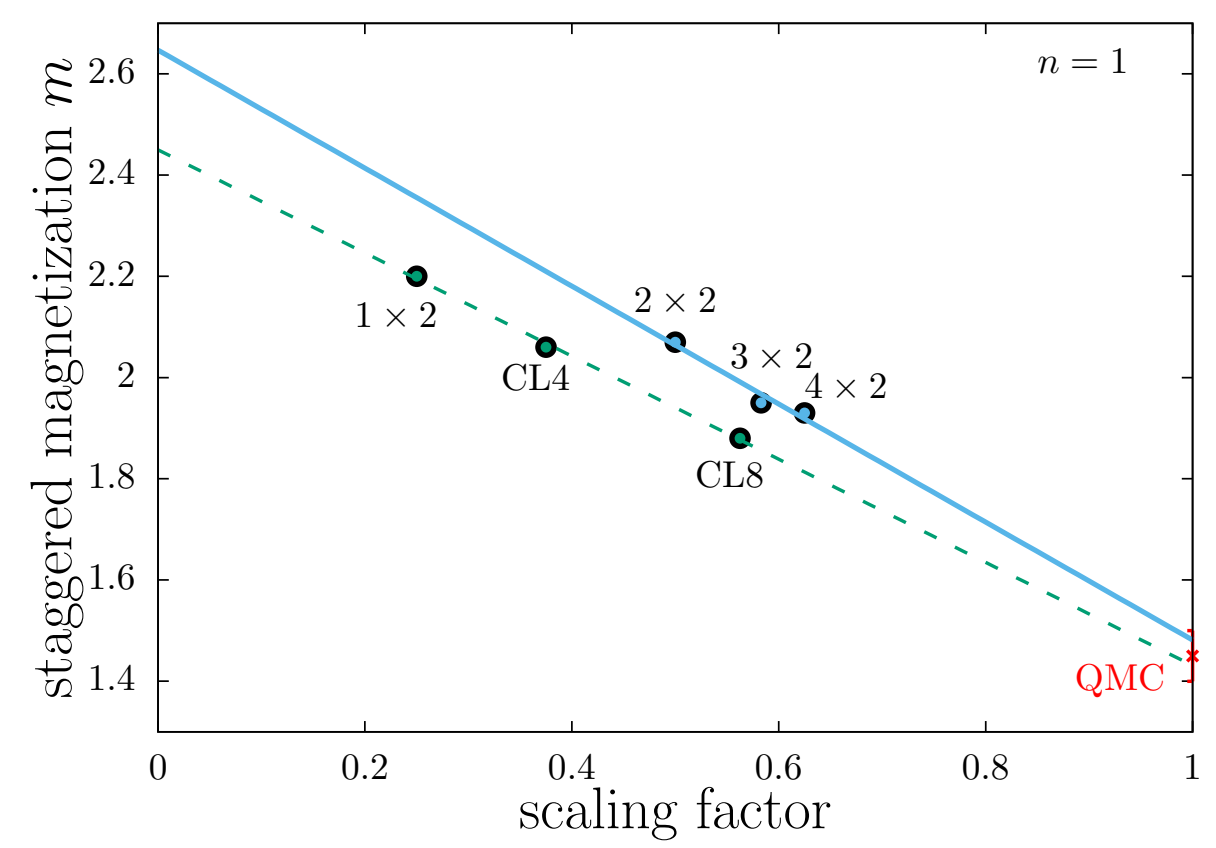

Figure 5.8: Attempts to finite-size-extrapolate the critical $J_{c} / t$ to the infinite-size cluster limit. The scaling factor equals the number of intra-cluster links devided by twice the number of cluster sites - a scaling factor of 1 amounts to the infinite-size-cluster limit. A fit to the $J_{c} / t$-values of the ladder clusters $N \times 2$ leads to a value $J_{c} / t=1.48 \pm 0.28$, which is close to the QMC result by Assaad [Ass99] (shown in red).

In order to do a finite-size scaling, the best approach would be to use clusters with different size which follow a systematic series, such as quadratic clusters. Unfortunately, the accessible clusters are limited to small clusters and one has to resort to other geometries, e.g. ladder geometries. In reference [Sen08b] Sénéchal compared different scaling factors and tested the outcome of a finite-size extrapolation in case of the Hubbard model on a two-dimensional square lattice, where larger clusters could be used due to the smaller local basis. The most promising scaling factor of this comparison, which is also applied in figure 5.8, consists in taking the number of links on the cluster and dividing it by twice the number of cluster sites. It scales to one in the limit of infinite cluster size and takes the ratio of boundary and bulk into account. 
Extrapolation by considering only the "ladder" clusters $2 \times 2,3 \times 2$ and $4 \times 2$ (blue fit in Fig. 5.8) results in an infinite-cluster size value of $J_{c} / t=1.48 \pm 0.28$. However, as quite pathological clusters as $1 \times 2$ and $C L 4$, which include dangling sites, have been used it is not a surprise, that the critical $J_{c}$ spread a lot depending on cluster geometry. In the end, larger clusters are needed to confirm the result of the ladder cluster extrapolation and improve this rough fit.

The critical value obtained by this rough fit is much improved compared to the one of $\mathrm{DMFT}+\mathrm{NRG}$. However, the reference value of $J / t=1.45 \pm 0.05$ is the one obtained via quantum Monte Carlo (QMC) by Assaad [Ass99], which is finite-size extrapolated and can be considered to be exact. Although extrapolated with a rough fit, the value of VCA agrees within error bars with this exact value. Recently, in a study by Otsuki the dual fermion technique was applied to the Kondo lattice model [Ots15]. Its value of the critical coupling strength also agrees with the QMC value and [Ots15] will be used as a reference for superconductivity in section 5.4.1 later.

\section{Ground State Analysis}

At half-filling QMC finds an insulator or more precisely a non-zero quasiparticle gap for all finite $J / t$ [Ass99]. A quantity that will also be used later to compare putative superconducting ground states is the local spin-spin correlator between conduction electrons and localized moments $\left\langle\mathbf{S}_{\mathbf{i}} \cdot \mathbf{s}_{\mathbf{i}}\right\rangle$. As will be shown in the following, this quantity is also useful to gain valuable insight in the nature of the ground state when not allowing for superconductivity.

Here, the ground state of a $4 \times 2$ cluster at various $J / t$ is analyzed by looking at this correlator, which is defined in $\mathrm{F}$ and shown in the left panel of figure 5.9. Due to the higher computational cost for the $4 \times 2$ cluster only $M_{c}$ has been used as a variational parameter. In the next subsection the influence of including the cluster hopping $t^{\prime}$ into the set of variational parameters will be discussed.
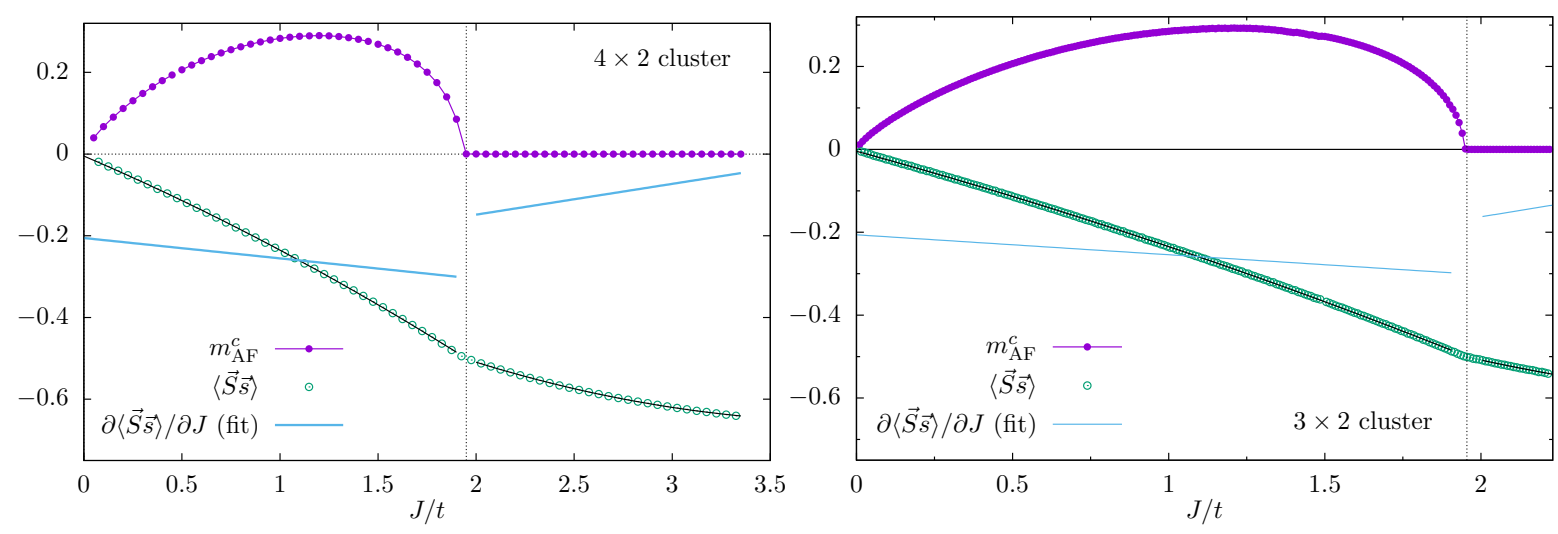

Figure 5.9: Staggered magnetization and expectation value of the Spin-spin correlator $\langle\mathbf{S} \cdot \mathbf{s}\rangle=$ $\partial \Omega / \partial J$ as a function of $J / t$ for a $4 \times 2$ (left) and a $3 \times 2$ (right) cluster at half-filling. Black lines are fits of the spin-spin correlator, blue lines denote their derivatives. They have been fitted with second order polynomials in the antiferromagnetic and paramagnetic regions up to $J_{c} / t \pm 0.05$. For the $4 \times 2$ cluster only $M_{c}$, for the $3 \times 2$ cluster both $M_{c}$ and $t^{\prime}$ were used as variational parameters. 
As far as the staggered magnetization and the transition from paramagnetic to antiferromagnetic insulator is considered, the spin-spin correlator seems to be a good measure of the transition point $J_{c}$ : At $J_{c}$ the gradient of $\left\langle\mathbf{S}_{\mathbf{i}} \cdot \mathbf{S}_{\mathbf{i}}\right\rangle$ jumps and its curvature changes sign. It is remarkable that the transition point $J_{c}$ coincides with a value of -0.5 of the spin-spin correlator. However, on the right side of figure 5.9 the same quantities are shown for a $3 \times 2$ cluster, this time also including the cluster hopping $t^{\prime}$ into the set of variational parameters. Figure 5.8 already showed that the $J_{c}$ values of both clusters are only slightly different. What is striking is the fact that also for the $3 \times 2$ cluster the transition seems to coincide with $\langle\mathbf{S} \cdot \mathbf{s}\rangle=-0.5$, where the curvature of the spin-spin correlator changes sign.

In the strong coupling limit the spin-spin correlator converges to a value of $-3 / 4$ per site which is expected for Kondo singlets. When approaching zero coupling, the correlator goes to zero as well, which means that electrons and f-spins are uncorrelated. In this sense, the spin-spin correlator shows the expected behavior in the limits of $J \rightarrow 0$ and $J \rightarrow \infty$. Away from this limits, the values differ from zero and $-3 / 4$. This is due to the fact that electron hopping not only counteracts the Kondo singlet ordering tendencies induced by the coupling term, but also mediates the effective RKKY interaction between the f-spins that causes the antiferromagnetic order.

For perfect Néel order, the f-spins and conduction electrons order and a spin-spin correlator of $-1 / 4$ per site would be expected as a staggered Weiss field breaks the local spin symmetry. At the transition to antiferromagnetic ordering the correlator shows a kink, but it is smooth below $J_{c}$ without evident kinks. That is surprising as the investigation of the paramagnet showed a breakdown of the insulator at $J \approx 0.8$. Simply assuming the paramagnetic contribution to be unchanged should naively result in a change of the ground state at roughly this coupling strength. However, the inclusion of antiferromagnetism allows for new many-body ground states, which means that the paramagnetic results cannot be transferred one-to-one to this situation. On the one hand, the onset of antiferromagnetism could destroy singlets and thereby cause a Kondo breakdown scenario at even larger coupling strengths $J \gtrsim 0.8$. On the other hand, it is not necessarily the case that singlets have to be broken up as one is far from a full singlet ground state and the magnetization never reaches saturation, ergo there would be enough "free" electrons to form the antiferromagnet. It is even possible to think of a scenario where the mobile electrons that counteracted the Kondo singlet formation in case of the paramagnetic study are now frozen out in an antiferromagnetic Néel state. In this case the breakdown of the paramagnetic insulator would be moved to weaker coupling or even avoided at all.

Assuming for $0<J<J_{c}$, that the ground state is a mixture of three different states, corresponding to a state without correlation between electron and f-spin, an antiferromagnetically ordered Néel state and a Kondo singlet state, clarifies the situation close to the transition: At least in the region with $\langle\mathbf{S} \cdot \mathbf{s}\rangle\langle-0.25$ a considerable admixture of Kondo singlets has to be present in the ground state. Even for small coupling strengths where the spin-spin correlator is larger than that, the absence of a kink for $J<J_{c}$ casts the occurrence of a sudden Kondo breakdown into doubt. However, considering only the spin-spin correlator in principle still renders two scenarios possible: Either Kondo singlets survive down to small coupling strengths within the whole antiferromagnetic phase or they smoothly decay when reducing the coupling strength.

As a first attempt one could assume that the contribution of antiferromagnetic states to the ground state is proportional to the staggered magnetization $m$. Without any further 
contributions, the spin-spin correlator should always stay non-positive when subtracting the contribution of antiferromagnetically ordered states. It is from the form of the magnetization curve already obvious that it is not possible to obtain a vanishing correlator for all coupling strengths up to some $J_{K}$, where Kondo singlet formation would set in, when subtracting the antiferromagnetic contribution in this form from the spin-spin correlator. Of course this is far from a proof as the true contribution of the antiferromagnetic part does not have to be proportional to the order parameter in this phase, but it at least renders the scenario of persistent Kondo singlets more plausible.
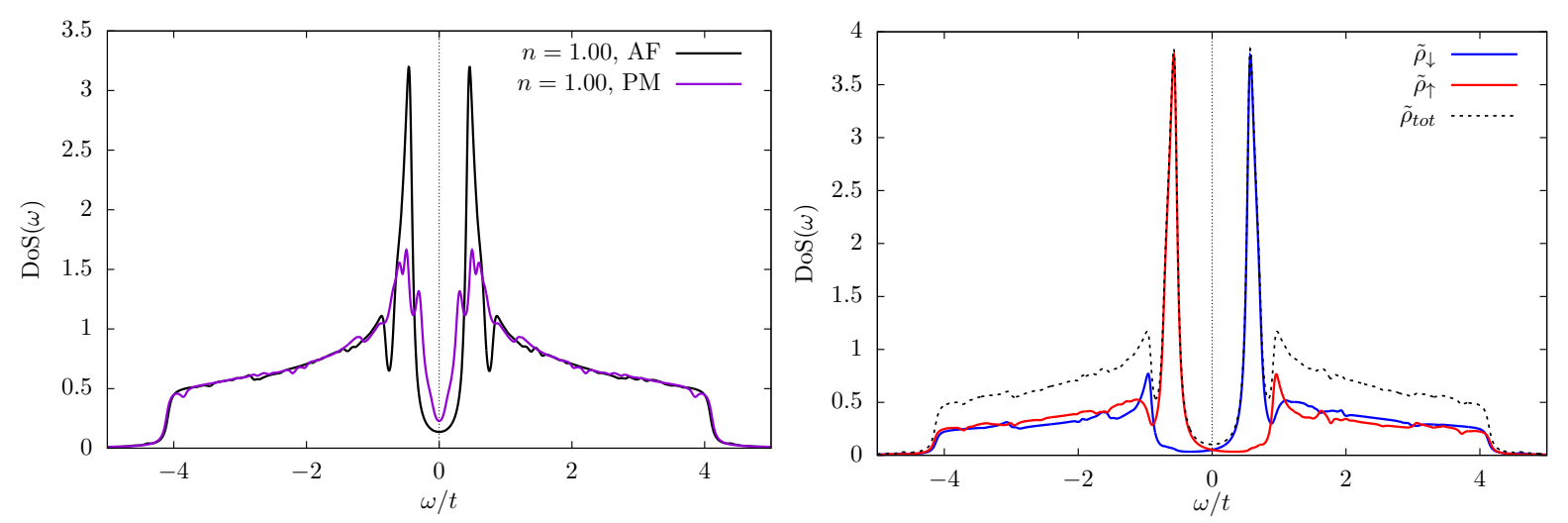

Figure 5.10: Left: Density of states (DoS) at $J / t=1.0$ and half-filling comparing the antiferromagnetic and paramagnetic insulator. Right: Composition of the antiferromagnetic total local DoS (dashed line) by up- and down-spin densities $\tilde{\rho}_{\uparrow}$ (red) and $\tilde{\rho}_{\downarrow}$ (blue), which are explained in the text. The DoS has been obtained on a $k$-grid of $100 \times 100$ points by using the $3 \times 2$ cluster and an artificial broadening of $\eta=0.05$.

Another quantity which shows the difference between the antiferromagnetic (AF) ground state and the paramagnetic alternative solution at half-filling for $J<J_{c}$ is the density of states (DoS). Although the paramagnetic (PM) solution is not realized at small coupling, both the AF and PM solution are compared at the same coupling strength to show several differences in the DoS that are characteristic for the AF and PM solution respectively. In figure 5.10, the DoS is shown at $J / t=1.0$ for both solutions. It has to be pointed out, that the DoS is obtained by integrating the spectral function $A(k, \omega)$ over the Brillouin zone. Here, the integration has been replaced by a summation over $k$-points of an equidistant two-dimensional grid of 10000 points that covers the Brillouin zone.

Furthermore, the $Q$-matrix formalism of VCA allows for the determination of the peak positions and corresponding weights of the spectral function. In order to make the deltapeaks visible, an additional artificial broadening of $\eta=0.05$ has been used for the spectral function and thereby also enters the DoS. As a consequence of the broadening, both solutions seem to have a finite DoS at the Fermi energy $\omega=0$. However, when reducing the broadening or analyzing the not broadened data both solutions show no states at the Fermi energy, but a quasiparticle gap instead.

Getting back to the structure of the DoS, the analysis of the DoS will from here on most of the times focus on the frequency window close to the Fermi energy, in this case $\omega \in(-1.5,1.5)$. Before turning to the peak structure in this region, it should be pointed 
out that due to the calculation of the Green function with the Band Lanczos method small contributions to the spectrum are lost. This becomes noticeable in the form of small asymmetries when comparing the electron- and hole-part of the spectrum. Half-filling is the particle-hole symmetric point of the (unfrustrated) KLM, hence perfect symmetry of the spectra would be expected. It also shows up in slight differences between the up- and down-spin part of the densities, which should be identical in the paramagnetic spectrum. Both of these discrepancies do not show up in the energy window close to the Fermi energy, but at larger energies. The features of the DoS that will be discussed in the following are therefore not affected.

Due to the particle-hole symmetry of the DoS at half-filling, the discussion will focus on the electronic part only. In case of the antiferromagnet, the DoS is characterized by a sharp resonance right at the border of the quasiparticle gap, followed by a side resonance at larger frequency. The paramagnet has a smaller quasiparticle gap and three distinct peaks can be identified. Compared to the antiferromagnet, where the main resonance was separated from the side resonance by a dip, the three peaks of the paramagnet are quite close to each other.

The paramagnetic DoS consists in equal parts of the DoS of electrons with up- and down-spin. In contrast, the antiferromagnetic DoS has different contributions for upand down-electrons when focusing on one of the sublattices A and B as shown in the right panel of figure 5.10. Due to the antiferromagnetic order the density of up-spin electrons on the A-sublattice has to be equal to the density of down-spin electrons on the B-sublattice. Therefore it makes sense to consider the staggered-average local DoS, $\tilde{\rho}_{\sigma}:=\frac{1}{N_{c}} \sum_{\mathbf{r}} e^{i \mathbf{r} \cdot \mathbf{Q}} \rho_{\sigma \mathbf{r}}$, to investigate the change of the DoS when approaching the phase transition $J \rightarrow J_{c}$. Still, at half-filling the total $\operatorname{DoS}=\tilde{\rho}_{\uparrow}+\tilde{\rho}_{\downarrow}$ is symmetric with respect to $\omega=0$, but the contributions $\rho_{\uparrow}$ and $\rho_{\downarrow}$ do not show this symmetry any more. In order to fulfill the symmetry of the total DoS the relation $\rho_{\uparrow}^{A}(\omega)=\rho_{\downarrow}^{A}(-\omega)$ holds at half-filling. At small interaction strength two pronounced main resonances (MR) at the band edges are visible, which are separated by the quasiparticle gap, and right next to them exist small side resonances (SR), see e.g. $J / t=0.8$ in figure 5.12. If one limits the discussion to the filled part of the DoS for one of the sublattices, the main resonance is mainly made up of the majority spin electrons and the side resonance predominantly of minority spin electrons, although with a considerable admixture of majority spin electrons. Increasing $J$ affects the main and side resonance differently: The weight of the main resonance first increases but then decreases again with a maximum at $J \approx 1.2$, which coincides with the maximal staggered magnetization. For $J>1.2$, the main peak starts to split into two peaks $\mathrm{MR}_{1,2}$, which have initially similar weight. When approaching the critical interaction strength $J_{c}$ the weight of the peaks diminishes and redistributes between $\mathrm{MR}_{1}$ and $\mathrm{MR}_{2}$ such that the peak $\mathrm{MR}_{1}$ closer to the side resonance retains more weight. The side resonance moves to larger frequencies when $J$ is increased and gains weight.

In figure 5.12 the peak position devided by $J$ is plotted against the coupling strength. Following the resonance $\mathrm{MR}_{1}$ to lower coupling insinuates that the main contribution of the joint peak for $J / t \lesssim 1.5$ stems from $\mathrm{MR}_{1}$ rather than from $\mathrm{MR}_{2}$.

Close to the transition the densities $\tilde{\rho}_{\uparrow}$ and $\tilde{\rho}_{\downarrow}$ converge to the same density DoS $/ 2$. At the transition, the side resonance of the minority electrons and $\mathrm{MR}_{1}$ of the majority electrons merge to one new side resonance and the resonance $\mathrm{MR}_{2}$ is made up equally from both spin-up and spin-down electrons.

This reshuffling of weight when approaching the phase transition reflects the competition 

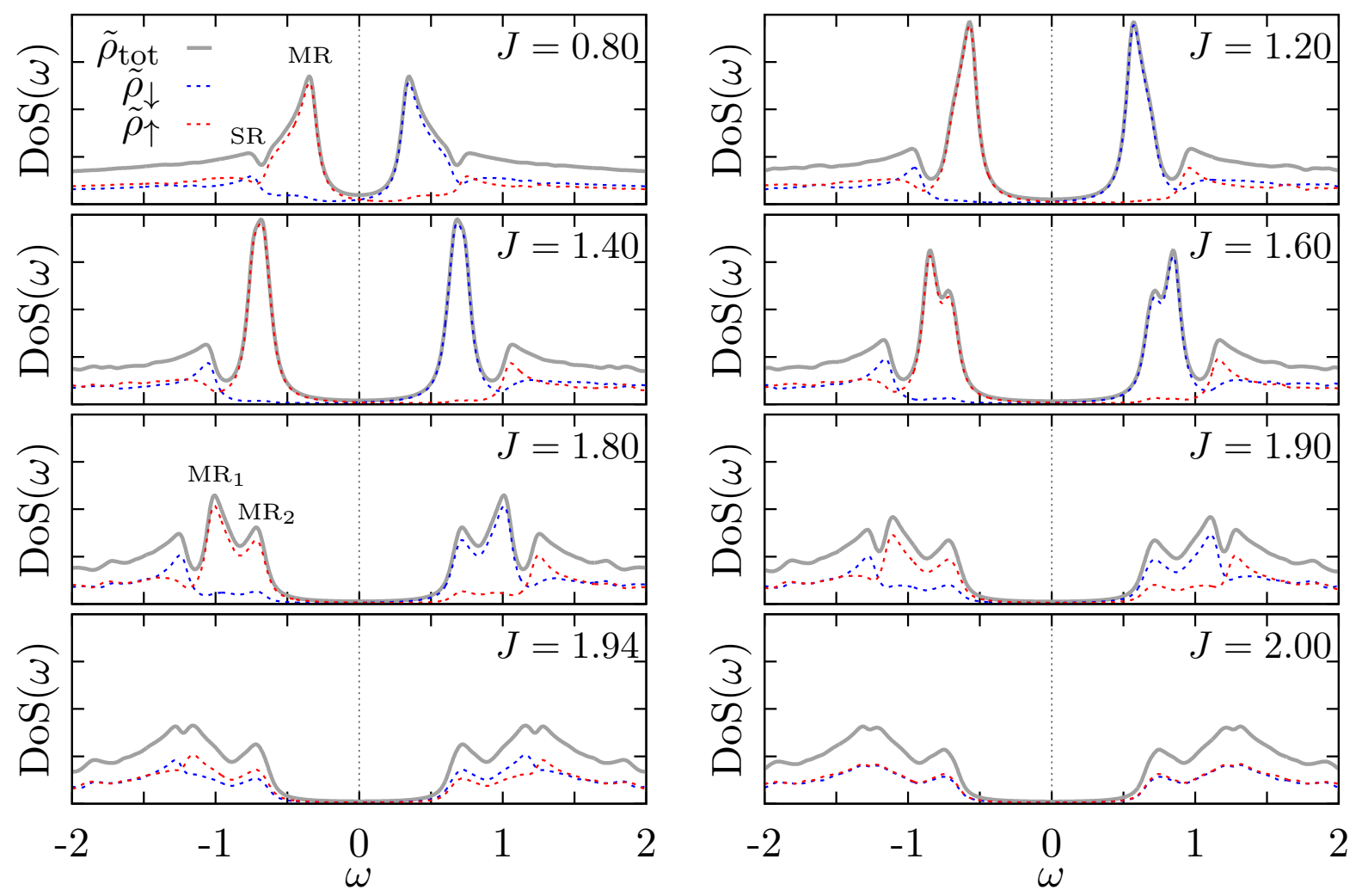

Figure 5.11: Density of states (DoS) at half-filling for several $J / t$ in the antiferromagnetic phase. The rest of the parameters are the same as in figure 5.10 and the scale of $\operatorname{DoS}(\omega)$ is kept the same for all panels. The total DoS $\tilde{\rho}_{\text {tot }}$ is made up by up- and down-spin densities $\tilde{\rho}_{\uparrow}$ (red) and $\tilde{\rho}_{\downarrow}$ (blue).

between the two different mechanisms that are responsible for the formation of a quasiparticle gap. In the antiferromagnetic region close to the transition there are already strong precursors of the paramagnetic insulator visible in form of a notable contribution of minority spin electrons to the peak at the gap edges.

Reconsidering the Kondo insulator at strong coupling which was briefly introduced in section 1.3.2, one can try to identify the peak which is responsible for Kondo singlet destruction. As the singlets have an energy of $-3 / 4 J$ the destruction of such a singlet by creating or annihilating a conduction electron comes with an energy cost of $3 / 4 \mathrm{~J}$ and should therefore show up in the density of states. Indeed, the energy of the merged resonances $\mathrm{MR}_{1}$ and $\mathrm{SR}$ roughly agrees with this energy at stronger coupling (for example at $J / t=3.0)$. When identifying the resonance with a Kondo singlet peak, it should thereby be possible to trace the existence of Kondo singlets even back to the antiferromagnetic phase.

As the antiferromagnetic order is far from perfect, there is no contradiction, but instead the ground state in the antiferromagnetic region can be interpreted as still having a finite amount of Kondo singlets. However, as the resonance splits up into two peaks close to the transition, which for weaker coupling strengths $J<J_{c}$ behave differently, it is not possible to clearly identify the existence of Kondo singlets in the antiferromagnetic region in this way. This will be discussed in more detail in subsection 5.2.2. 


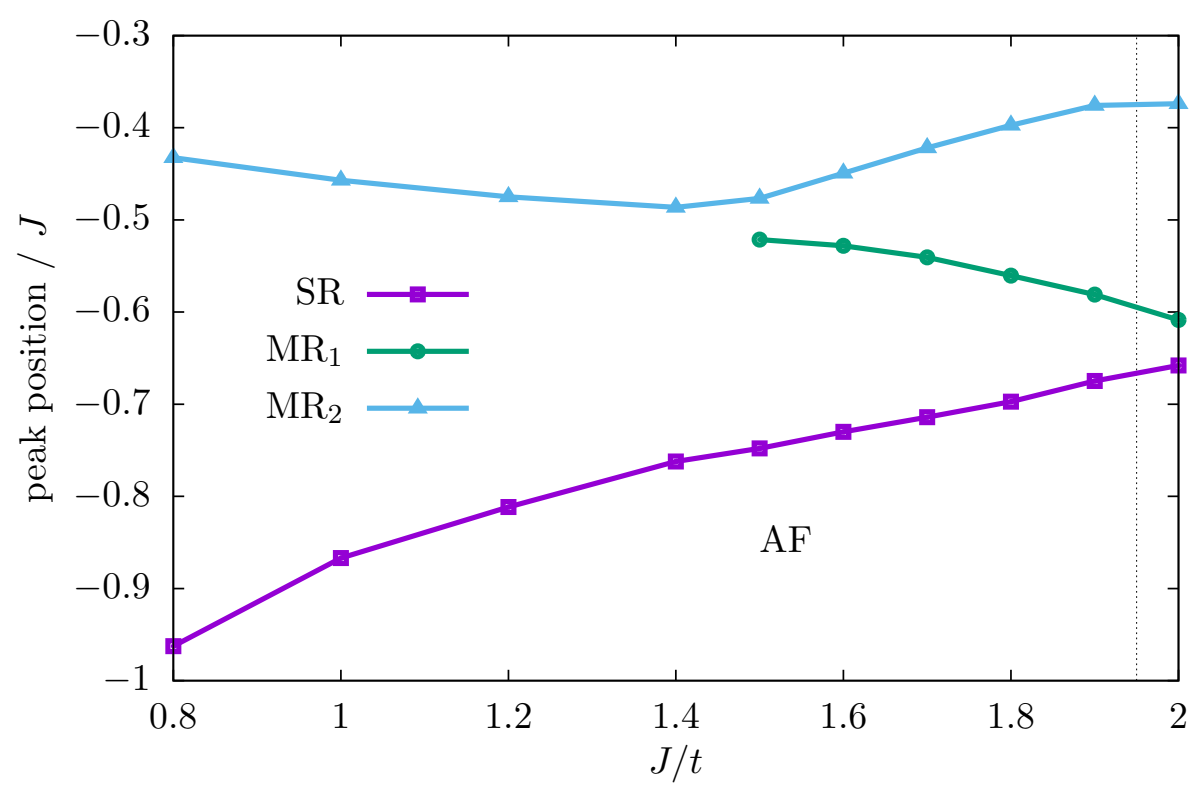

Figure 5.12: Relative position of the resonances $\mathrm{MR}_{1}, \mathrm{MR}_{2}$ and $\mathrm{SR}$ in the density of states defined in figure 5.12. For values $J<1.5$ the two peaks $\mathrm{MR}_{1}$ and $\mathrm{MR}_{2}$ can not be distinguished any more and only one peak position is shown.

\section{Doping the Antiferromagnet $\mid 5.2 .2$}

The situation in the half-filled Kondo lattice model is quite special: In the ground state, every local moment can be screened in the large $J$ limit with exactly one electron to form a singlet at each site. As already mentioned in section 2.3, removing conduction electrons from the half-filled system creates unpaired local moments which can be interpreted as spinfull c-holes. In the paramagnetic case, Kondo singlets can still be formed for sufficiently large Kondo coupling, although the electrons gain mobility due to vacancies in the conduction band, see section 5.1.3. For smaller values of $J$ doping the system is also interesting as the number of electrons which mediate the antiferromagnetic order of the local spins via RKKY interaction is then reduced. Naively, one would hence expect, that the antiferromagnetic correlations diminish when the system gets doped.

From the previous subsections it is known that the ground state for a $3 \times 2$ cluster is antiferromagnetically ordered in the half-filled case for $J<J_{c} \approx 1.95$, see for instance figure 5.9. In this region, one expects that the antiferromagnetic ordering will be destroyed at a sufficiently large doping as the conduction electrons, which function as mediators for the RKKY interaction between the local moments are partly removed. The focus is set on an intermediate Kondo coupling of $J=1.6 t=0.2 \mathrm{~W}$ at which we can directly compare with DMFT results of reference $\left[\mathrm{BŽV}^{+} 13\right]$.

The left panel of figure 5.13 compares the energy of the antiferromagnetic and paramagnetic solutions as a function of electron filling. It can be seen that the antiferromagnetic solution exists down to a critical electron filling of $n_{c} \approx 0.915$. When approaching this filling from above, the staggered magnetization of the AF solution vanishes continuously and the corresponding energy approaches the one of the PM solution. As its energy is always lower than the one of the paramagnet, the antiferromagnetic metallic phase is realized close to half-filling and continuously goes over to a paramagnetic phase when 

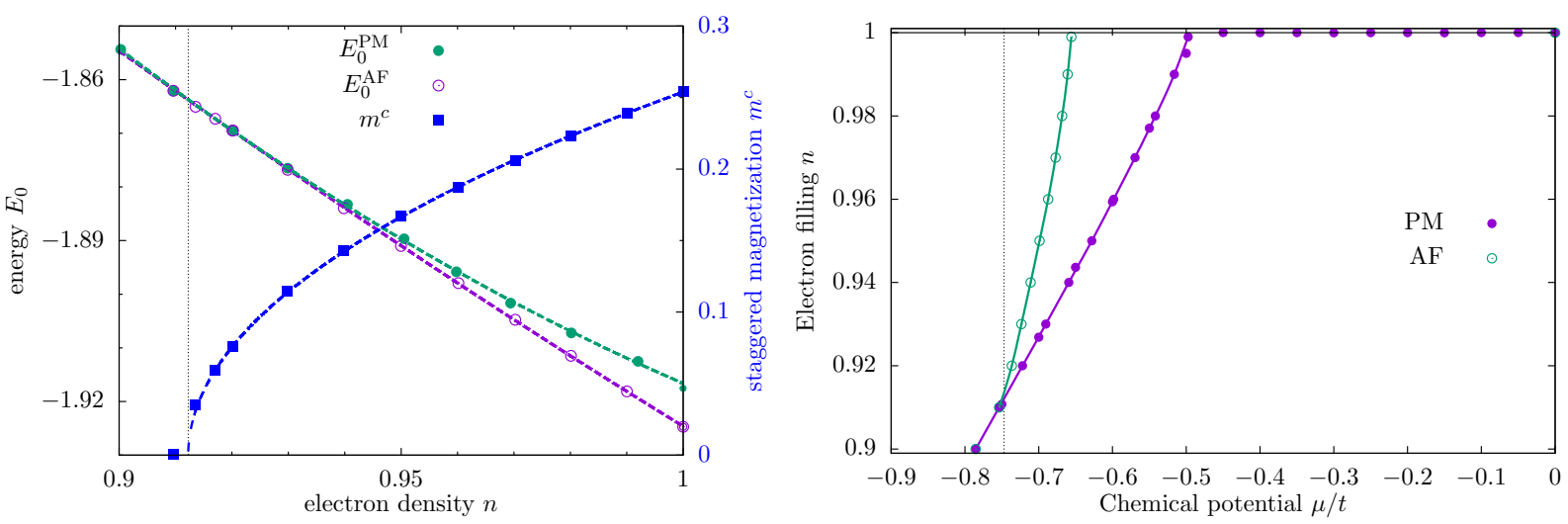

Figure 5.13: Left panel: Comparison of the normal/paramagnetic (PM) and the antiferromagnetic (AF) solution at $J / t=1.6$ as a function of electron filling $n$. For $n \geqslant 0.91$ the antiferromagnetic solution is energetically preferred, but at $J_{c}^{\mathrm{AF}}$ the staggered magnetization $m^{c}$ is zero and the solution is therefore paramagnetic. The dashed line is a square root fit to the staggered magnetization data. Right panel: Electron filling $n$ at $J / t=1.6$ as a function of chemical potential $\mu$ as obtained with a $3 \times 2$ cluster. Two solutions are shown: One including an antiferromagnetic Weiss field ('AF'), the other one showing the paramagnetic solution for comparison. In the region $\mu<\mu_{c} \approx-0.74$ only the paramagnetic solution is realized, but for $\mu>\mu_{c}$ the antiferromagnetic solution has lower energy. Lines are guides to the eye only.

approaching $n_{c}$.

In a DMFT study by Otsuki et al. [OKK09] the critical electron density down to which the antiferromagnetic ground state holds for a Kondo coupling of $J=0.2 \mathrm{~W}$ was found to be $n_{c}(J=0.2 W) \approx 0.9$. As will be shown in section 5.2.3 including more than one (isotropic) hopping parameter of the cluster into the set of variational parameters leads to a slight change of $n_{c}$, which is why the comparison with DMFT will be discussed there.

The right panel of figure 5.13 shows the electron density $n$ as a function of chemical potential $\mu$ for both the antiferromagnetic and the paramagnetic solution and one immediately notes the change in $n$-vs. $-\mu$ when including antiferromagnetism. The paramagnet shows a plateau corresponding to zero compressibility which indicates an insulator and introducing an antiferromagnetic Weiss field correctly produces an antiferromagnetic insulator at half-filling, which has a larger quasiparticle gap. In the paramagnetic case the quasiparticle gap turned out to be too small in the region $J<J_{c}$ when compared to QMC (see figure 5.4). Although the gap of the antiferromagnetic solution is larger, unfortunately the gap depends stronger on the size of the cluster than the gap of the paramagnetic solution for $J>J_{c}$ which makes a comparison with the QMC data difficult. When considering only the data of the $3 \times 2$ cluster, the quasiparticle gap is too large and a finite-size scaling of the gaps would be necessary, which in the half-filled case already showed indications for reproducing the exact critical coupling strength $J_{c}$.

In figure 5.14 the staggered magnetization and the spin-spin correlator are shown at $n=$ 0.95 as a function of coupling strength. The antiferromagnetic region $\left(J / t<J_{c} / t \sim 1.72\right)$ is found to be split into two different phases at $J / t=1.24$. Two stationary points exist for $J / t=1.24$ and the corresponding solutions differ in their staggered magnetization indicating a discontinuous transition between two antiferromagnetic phases, in the following denoted by $\mathrm{AF}_{1}$ and $\mathrm{AF}_{2}$, see figure 5.14. The discontinuous nature of the transition 


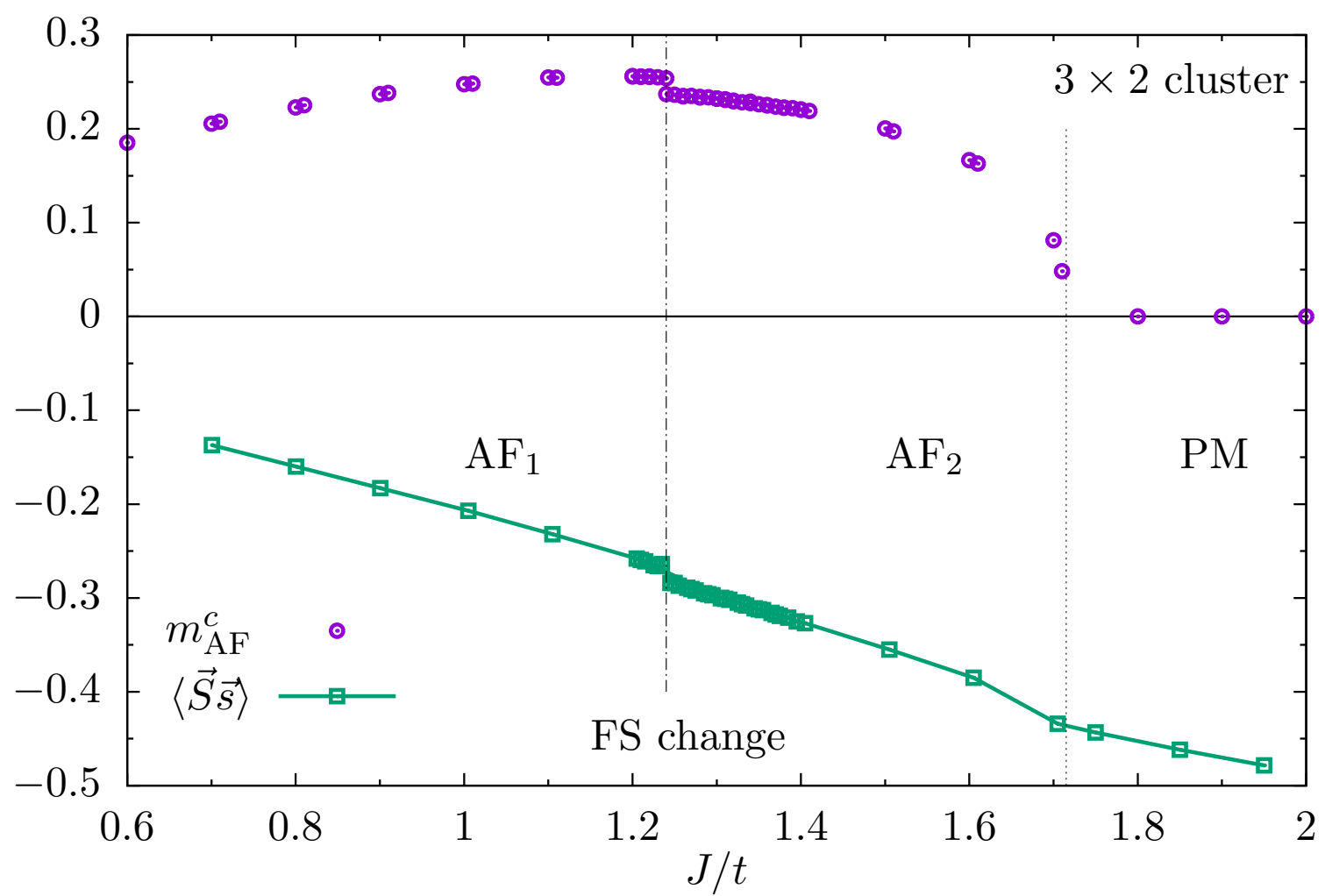

Figure 5.14: Staggered magnetization (top) and spin-spin correlator (bottom) of the antiferromagnetic (AF) solution as a function of coupling strength $J / t$ at $n=0.95$ using a $3 \times 2$ cluster with variational parameters $\mu, \mu^{\prime}, t^{\prime}$ and $M_{c}$. As can be seen from the jump in staggered magnetization at $J / t=1.24$ the system shows a first order transition between two antiferromagnetic phases.

also shows in a jump of the spin-spin correlator. In the following it is shown that the two phases differ in their Fermi surface topology.

To summarize the findings discussed so far, figure 5.15 shows the magnetic phase diagram close to half-filling using a $3 \times 2$ cluster. The grey regions denote paramagnetic solutions and the antiferromagnetic region is shown in blue, where the staggered magnetization is color coded. At half-filling, the data corresponds to the one already shown in figure 5.21 where only one cluster hopping parameter was varied. There, the chemical potentials of the system and the cluster had not to be varied and $\mu=\mu^{\prime}=0$.

Besides the magnetic properties of the system it is also interesting to investigate the metal-insulator transition as a function of electron density $n$. In the half-filled case it was shown in the previous subsection that the antiferromagnetic insulator goes over to an Kondo insulator at $J_{c}$. When doping the paramagnetic (Kondo) insulator it was also shown that the system turns metallic once the system is doped away from half-filling. Here, the "new" transitions are the one from an antiferromagnetic insulator to an antiferromagnetic metal for $J<J_{c}$ at $n=1 \rightarrow 1-\epsilon$ and from an antiferromagnetic to a paramagnetic metal for $J<J_{c}$ at $n_{c, \mathrm{AF}}$. The difference between these three phases can be nicely seen when looking at the density of states (DoS) in figure 5.16. It shows the DoS for $J / t=1.6$ at three different electron densities that are indicated by red cir- 


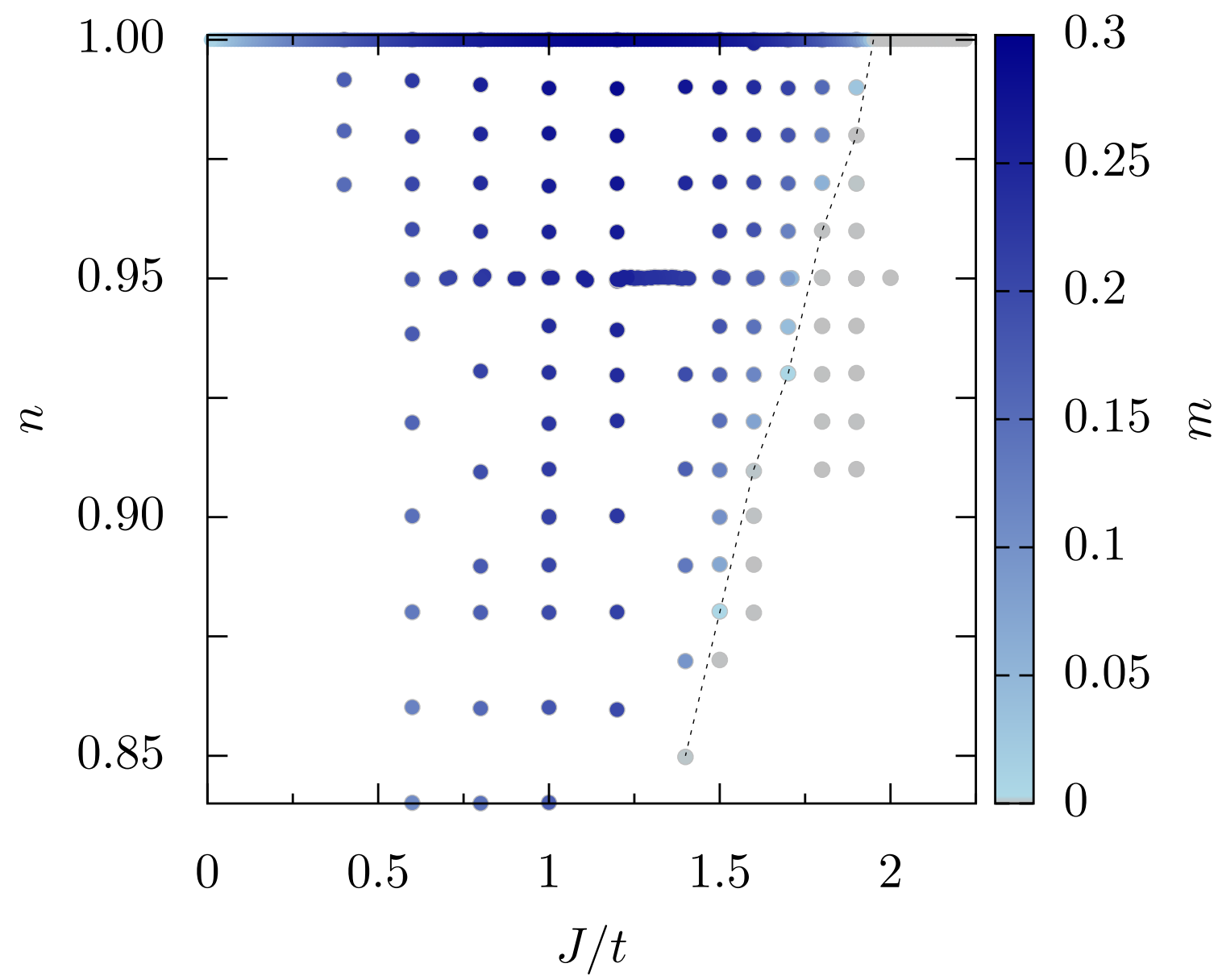

Figure 5.15: Staggered magnetization of the antiferromagnetic (AF) solution as a function of electron filling $n$ and $J / t$ using a $3 \times 2$ cluster with variational parameters $\mu, \mu^{\prime}, t^{\prime}$ and $M_{c}$. Circles denote data points, the value of the staggered magnetization $m$ is color-coded. The dashed line denotes the continuous phase transition between AF and PM metal.

cles in figure 5.22 and that correspond to the antiferromagnetic insulator $(n=1.0)$, the antiferromagnetic metal $(n=0.95)$ and the paramagnetic metal $(n=0.90)$.

In order to make the difference between antiferromagnetic metal and paramagnetic metal better visible, figure 5.16 shows the DoS at $J / t=1.6$ for different electron densities $n$. In contrast to figure 5.12, where the transition from AF to PM was studied at half-filling as a function of coupling strengths, the spectrum is not particle-hole symmetric. Still, the redistribution of the electron density between up- and down-electrons when approaching the magnetic order-disorder transition is similar. The relative position of the three peaks does not change much when reducing the electron filling, but their size changes from the pronounced two main resonance structure known from the half-filled case to a roughly equally sized three-peak structure close to the transition. Already at $n=0.99$ the main resonances are not entirely made up from the majority spins, but also carry little weight from the minority spins.

Again, the third peak SR is a candidate for a Kondo singlet peak: The difference between the peak position and the middle of the gap at positive frequencies is at $n=0.99$ roughly 1.15 which is close to the value of $3 / 4 J=1.2$. When doping further away from half-filling the relative peak position changes and differs more and more from $3 / 4 J$. This is somewhat 

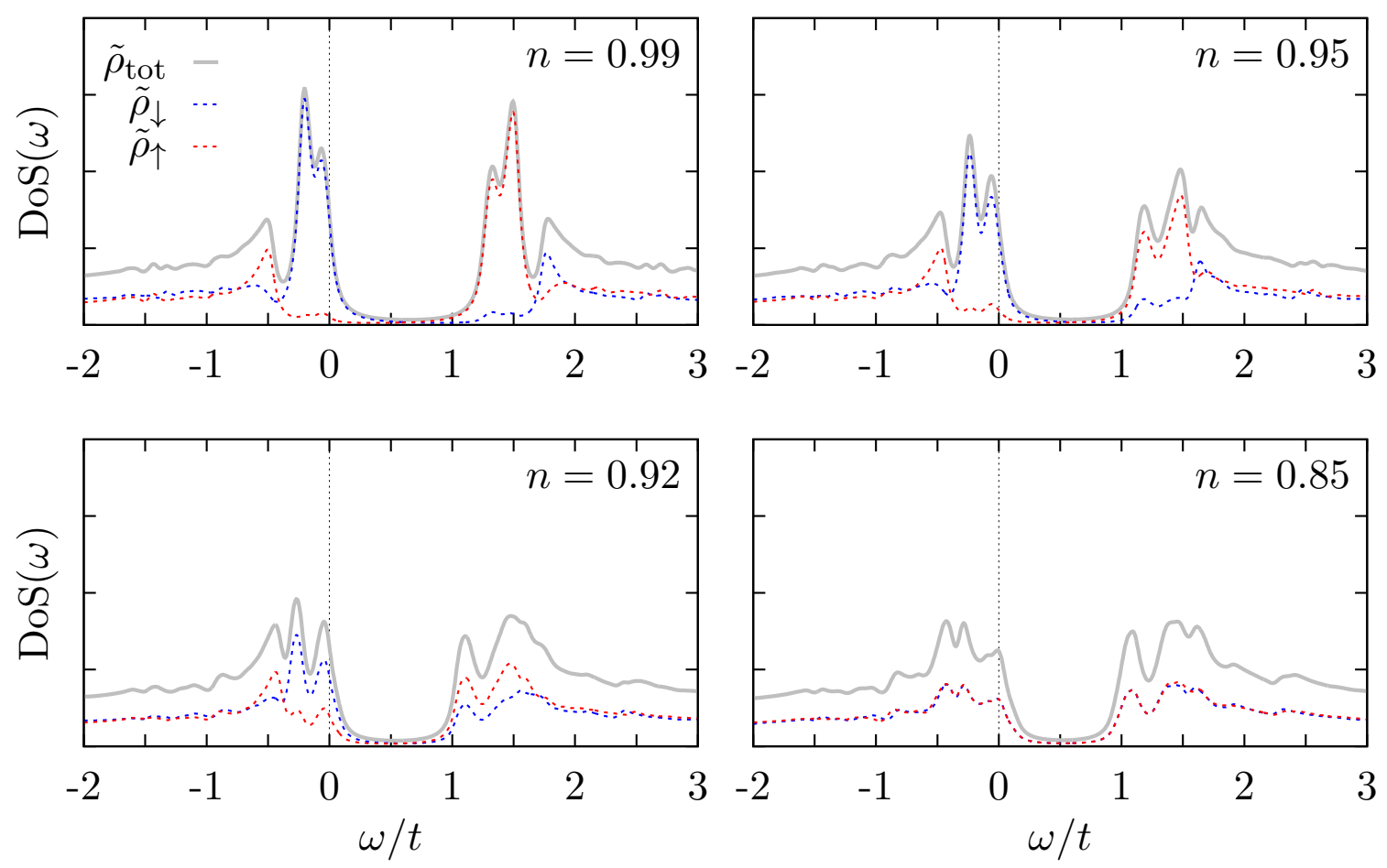

Figure 5.16: Density of states (DoS) for $J / t=1.6$ using the $3 \times 2$ cluster with $\left\{\mu, \mu^{\prime}, t^{\prime}, M_{c}\right\}$ as variational parameters. The electron fillings $0.91<n<1$ correspond to the antiferromagnetic metal phase and the first three panels show fillings close to the antiferromagnetic insulator $(n=0.99)$, close to the paramagnetic metal $(n=0.92)$ and right in the middle of the phase $(n=0.95)$. In the last panel $n=0.85$, which is deep in the paramagnetic metal phase. The DoS has been obtained on a $k$-grid of $100 \times 100$ points and an artificial broadening of $\eta=0.05$ has been added.

expected as the value of $3 / 4 J$ was obtained by assuming a perfect Kondo insulator. Once electrons are removed even at strong coupling the singlets are mobile and the resulting dispersion changes. Finally, in the paramagnetic region electrons of both spins contribute at each energy equally to the density of states.

Figure 5.17 shows the position of the resonances in the DoS at fixed electron density to $n=0.95$ as a function of $J / t$. In order to compare the positions at different coupling strengths their relative position with respect to the middle of the gap in the DoS has been devided by the coupling strength. At a value of $J / t=1.24$ the trend of all peak positions changes. This is due to the first order transition into a different antiferromagnetic phase that was shown already in figure 5.14. Compared to figure 5.12 where the peak positions are shown at half-filling, the change in the side resonance SR is much stronger. When extrapolating the trend of the position of the merged resonance ' $\mathrm{MR}_{2}$ ' at small coupling to couplings $J / t>1.24$ one ends up at the position of the resonance ' $\mathrm{MR}_{1}$ '. In contrast to the half-filled case, the merging of the two resonances at $J / \approx 1.25$ seems to coincide with a first order phase transition. At half-filling the antiferromagnetic insulator does not show any jump or kink in the staggered magnetization which indicates the importance of a change in the Fermi surface for the first order transition off half-filling. 


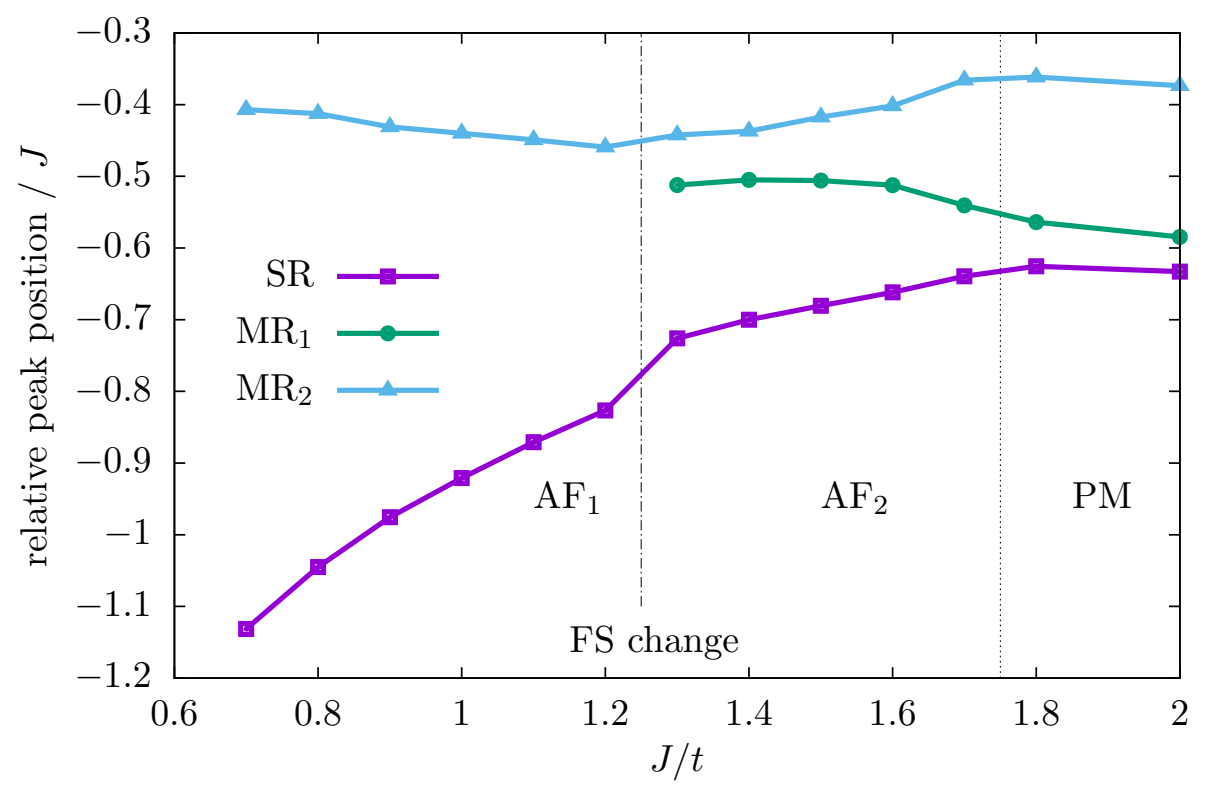

Figure 5.17: Relative positions of the resonances 'MR ${ }_{1}$, ' $M R_{2}$ ' and 'SR' with respect to the center of the gap at filling $n=0.95$ as a function of coupling strength, for more details see in the text. For $J / t<1.3$ the resonances ' $\mathrm{MR}_{1}$ ' and ' $\mathrm{MR}_{2}$ ' can not be distinguished any more.

The redistribution of weight inside the density of states is also reflected in the composition of the Fermi surface, which is discussed in the following subsection.

\section{Changing Fermi Surface Area}

Once the system is doped away from half-filling it is metallic and hence possesses a Fermi surface. Deep in the paramagnetic phase (strong coupling $J$ ) the Fermi surface has been briefly discussed in subsection 5.1.3 and its large area was explained by taking into account that both electrons and f-spins form the FS in form of mobile Kondo singlets.
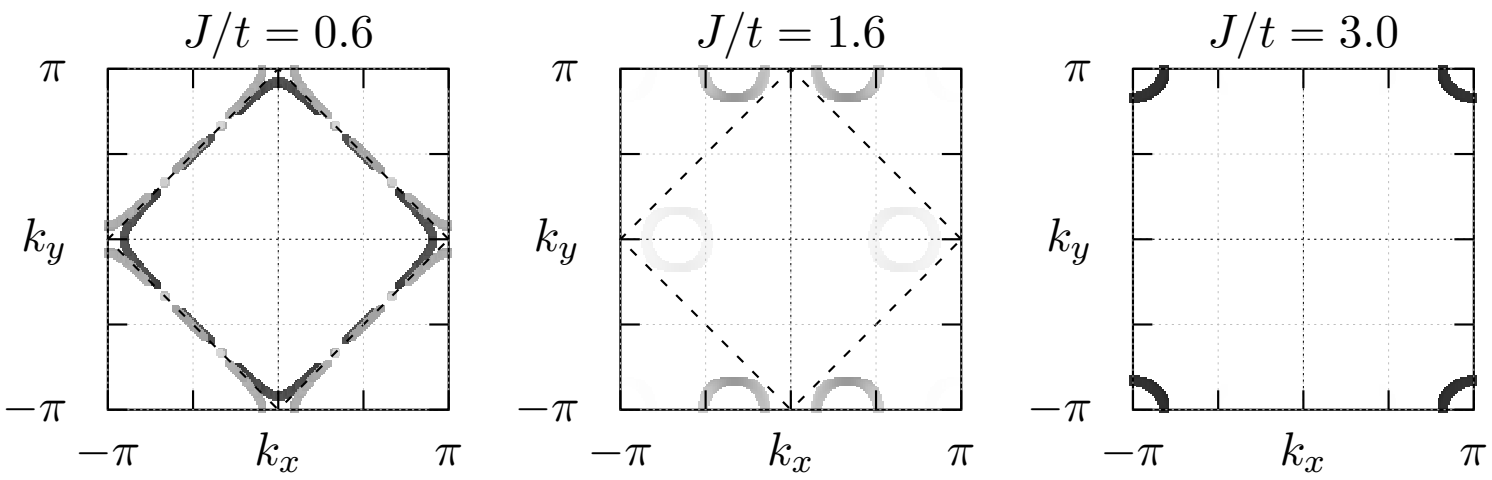

Figure 5.18: Characteristic Fermi surfaces (FS) for the three regimes that can be identified off half-filling, here shown is an electron density of $n=0.95$. At weak coupling the antiferromagnetic metal has a small Fermi surface (left panel), at intermediate coupling the Fermi surface volume jumps to a much higher value, but the system stays antiferromagnetic (center panel) and at strong coupling the system is paramagnetic with a large Fermi surface (right panel). 
Figure 5.18 shows characteristic Fermi surfaces close to half-filling $(n=0.95)$ for three different regimes. Besides the already described paramagnetic case, one can identify two distinct phases by looking at the Fermi surface of the resulting metallic solutions. In the antiferromagnetic phase the Brillouin zone doubles, which is in the figure indicated by a dashed line. At small coupling strengths, when focusing on the inner sheet of the Fermi surface it resembles the small closed Fermi surface that is found in the weak coupling region within other numerical techniques such as Dual Fermions [Ots15], RDMFT [PK15] or variational Monte Carlo [WO07, ABF13]. For larger coupling strengths close to the transition to the paramagnet the Fermi surface topology is different (middle panel of figure 5.18). Small closed structures appear in the Fermi surface, but the doubling of the Fermi surface that corresponds to antiferromagnetic long-range order still persists.

It is this very region close to the transition to the paramagnet where the spin-spin correlator at half-filling clearly indicated a considerable admixture of Kondo singlets in the ground state. At filling $n=0.95$ this is still the case, but at $J / t=1.24$ the correlator jumps to a higher value that is only a bit smaller than $-1 / 4$, see figure 5.14 . This indicates that at least a small admixture of Kondo singlets is still present in the antiferromagnetic phase $\mathrm{AF}_{1}$ close to the transition. As the spin-spin correlator is larger than $-1 / 4$ for weak coupling strengths, other quantities have to be analyzed in addition to resolve the question whether Kondo singlets exist even down to coupling strengths of the order of $J / t=0.6$, where a small Fermi surface is found.

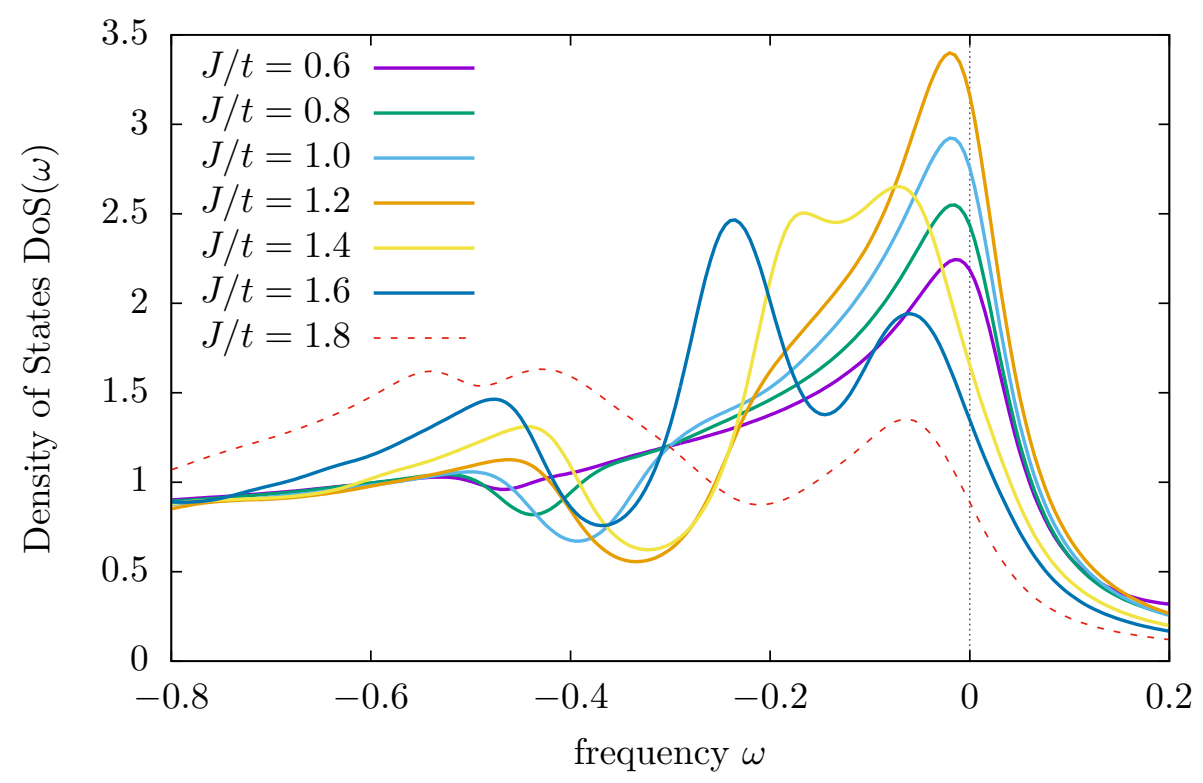

Figure 5.19: Density of states at $n=0.95$ for different coupling strengths in the antiferromagnetic $(J<1.7)$ and the paramagnetic $(J=1.8)$ region. A change of the peak structure close to the Fermi energy at $\omega=0$ happens at $J / t \gtrsim 1.2$ : The main peak splits and an additional side resonance develops. Close to $J_{c}$ the structure already resembles the three peak structure known from the Kondo insulating region for $J>J_{c}$.

To further study whether the two regions in the antiferromagnetic metal amount to a Kondo breakdown or whether Kondo singlets are still present in $\mathrm{AF}_{1}$, the density of states within the AF metal is revisited with a focus on the evolution of the peak 'SR'. If the Kondo breakdown picture applies, the small Fermi surface at weak coupling would be made up entirely by electrons whereas Kondo singlets would not exist. 
Figure 5.19 shows part of the DoS close to the Fermi surface for different values inside the AF phase at $n=0.95$. At the largest shown coupling strength close to critical coupling strength of $J_{c} / t \approx 1.75$, three distinct peaks are visible which are similar to the characteristic three peak structure of the paramagnet close to the transition $\left(J>J_{c}\right)$. When reducing $J$ the two peaks closest to the Fermi energy approach each other and finally seem to merge at $J / t \approx 1.2$. However, the bipartite character of this main resonance can be still seen in a shoulder at $\omega \approx-0.18$. Further decreasing the coupling strength results in a more and more broadened shoulder which at small coupling strengths such as $J / t=0.6$ can only be guessed to still exist.

More importantly, the leftmost peak which was motivated to be an indicator for Kondo singlet formation at half-filling survives even down to small coupling strengths. The peak height is reduced for decreasing coupling strength, but it is difficult to quantify the peak height from the DoS. To obtain the weight of this peak, it is necessary to remove the background which is a priori unknown. Defining the difference between the absolute height of the peak and the dip in the DoS right next to it at higher frequency has the disadvantage that it also depends on the position of the middle peak ('MR ${ }_{1}^{\text {'), }}$ which changes noticeably between $J / t=1.6$ and $J / t=1.2$. This might be due to the fact that the staggered magnetization is largest around $J / t \approx 1.2$. Therefore, an extrapolation of the (effective) peak height to even smaller coupling strengths is difficult. Still, a distinctive peak is visible at $J / t=0.6$, where the antiferromagnet shows the characteristic small Fermi surface. When taking the side resonance as an indicator for Kondo singlets, the DoS would suggest evidence for the existence of Kondo singlets at small coupling strengths.

However, the paramagnet close to the transition to magnetic order shows two peaks with nearly the same weight which merge for larger coupling strengths and are hence both candidates for Kondo singlet resonances. Following the other resonance ' $\mathrm{MR}_{1}$ ' when decreasing the coupling strength leads to the afore mentioned merging with peak ' $\mathrm{MR}_{2}$ ' at $J / t \sim 1.2$, where the Fermi surface topology changes. Therefore, taking the resonance ' $\mathrm{MR}_{1}$ ' as an indicator for Kondo singlets would rather suggest an absence of Kondo singlets at weak coupling. A definite answer to the question of existence or absence of Kondo singlets based on the peak structure of the DoS is therefore barely possible.

In figure 5.20 a close-up of the spectral function around the Fermi energy is shown at the same filling for different coupling strengths. Although it shows only a certain path through the Brillouin zone, the different regions of the Brillouin zone (BZ) that contribute to the Fermi surface at zero frequency can be identified. Due to the symmetry with respect to $\mathrm{x}-$ and y-direction, it is sufficient to only show this path of the BZ that for instance does not include the point $(0, \pi)$. Especially when changing from $J / t=0.6$ to $J / t=1.2$, where the DoS already showed a shoulder next to the main resonance, the spectral function seems to change continuously and the same features around $\omega=0$ are found. When approaching the paramagnetic phase, the spectral weight on the path $(\pi, 0)-(\pi / 2, \pi / 2)$ changes the most. Only little weight is left close to $\omega=0$ at $(\pi / 2, \pi / 2)$, instead weight is transferred to $(\pi, \pi / 2)$. Finally, in the paramagnetic phase the Fermi surface centers around $(\pi, \pi)$.

To conclude, it is difficult to fully exclude the Kondo breakdown scenario for very small coupling strengths. The density of states, the spin-spin correlator and spectra clearly indicate, that in the antiferromagnetic region close to $J_{c}$ Kondo singlets make part of the charge carriers. Based on this, a local quantum critical point, where Kondo breakdown and onset of antiferromagnetic order coincide at $J_{c}$, would be ruled out. The topological 

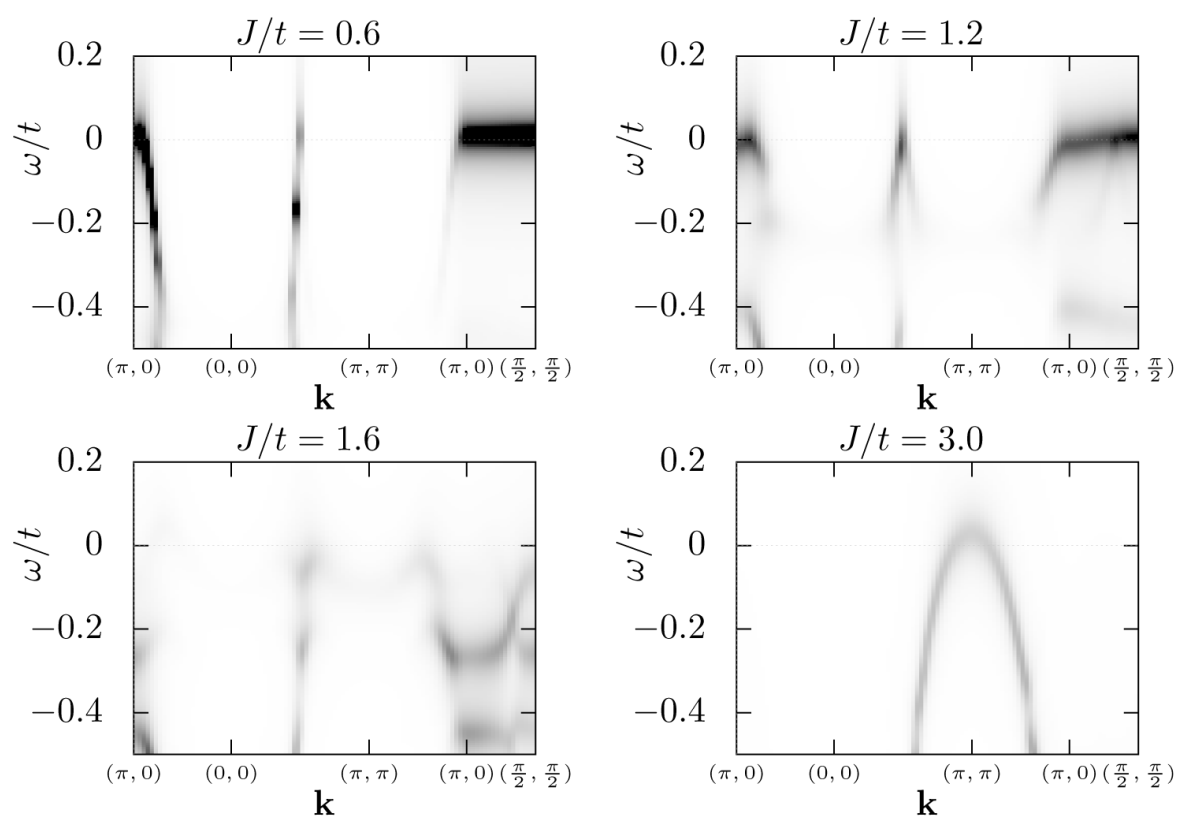

Figure 5.20: Close-up of the spectral function around the Fermi energy at $n=0.95$ for different coupling strengths in the antiferromagnetic region. A change of the structure close to the Fermi energy happens between $J / t=1.2$ and $J / t=1.6$.

differences between the Fermi surface in the weak coupling and intermediate coupling regime are also evident. However, ruling out a Kondo breakdown at the discontinuous transition between $\mathrm{AF}_{1}$ and $\mathrm{AF}_{2}$ is more difficult. At least close to the transition the value of the spin-spin correlator in phase $\mathrm{AF}_{1}$ indicates the presence of Kondo singlets. By using larger clusters it would be interesting to check, to what amount finite-size effects enter in the FS structures that have been discussed here and how the peaks ' $\mathrm{MR}_{1}$ ' and 'SR' evolve as a function of cluster size. It also has to be noted that other techniques which work directly in $k$-space, such as DCA [MA08, MBA10] or RDMFT [PK15], might be able to investigate the Fermi surface evolution more precisely.

The fact that a first-order phase transition is found between the antiferromagnetic phases with different Fermi topology agrees with variational Monte Carlo studies [WO07, ABF13]. Techniques that are based on dynamical mean-field theory find a (continuous) Lifshitz transition instead [MA08, PK15].

However, it should be noted that these methods are conceptionally different as they include temporal fluctuations to bath sites. Therefore, a study of the transition between the antiferromagnetic phases $\mathrm{AF}_{1}$ and $\mathrm{AF}_{2}$ via $\mathrm{VCA}$ including bath sites seems promising for future studies.

\section{Variation of the Intra-Cluster Hopping $t^{\prime}$}

As it proves important to include the intra-cluster hopping $t^{\prime}$ into the set of variational parameters when investigating superconductivity, its influence on antiferromagnetism is also investigated. Indeed, when including $t^{\prime}$, the minimum of the self-energy functional with respect to the staggered Weiss field $M$ changes a bit.

Starting off with the half-filled case and the $3 \times 2$ cluster, $t^{\prime}$ stays close to $t$ within 
the antiferromagnetic phase and hence the magnetization curves are not affected much. For the $2 \times 2$ cluster, at large $J / t$ the intra-cluster hopping is close to $t$, but this changes drastically when lowering $J / t$. This also affects the value of $M$ which is reduced compared to the value when fixing $t^{\prime}=t$. Interestingly, the magnetization curves $m_{c}$ and $m_{f}$ are not much affected. In the limit $J / t \rightarrow 0$ this can be understood as VCA becomes exact in the non-interacting limit. The self-energy is there $\Sigma(\omega)=0$, which is why large intra-cluster hopping does not change the outcome of the calculation much.

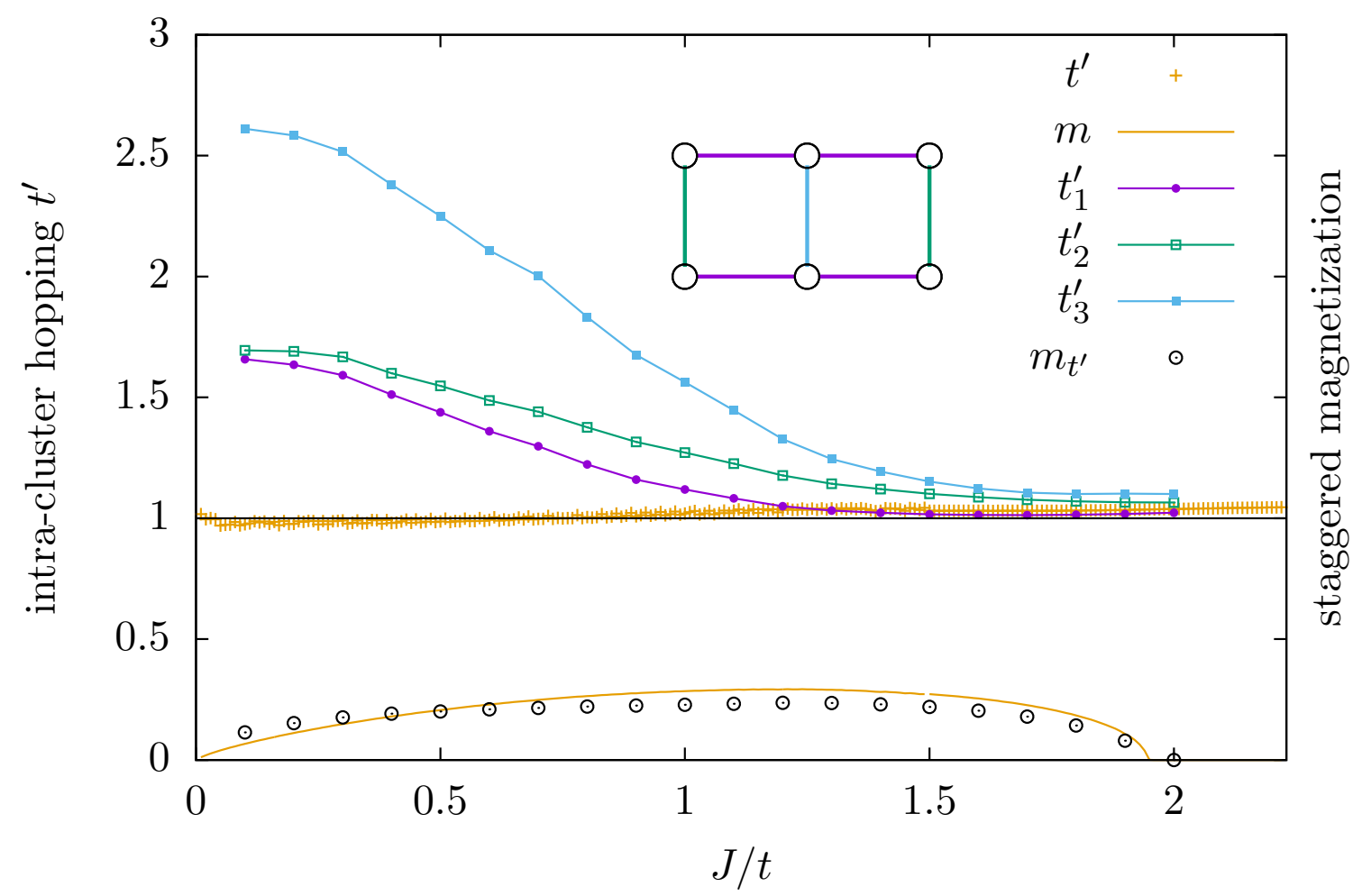

Figure 5.21: Variation of the three different hopping parameters in the $3 \times 2$ cluster leads to larger deviations from $t$.

One might also ask for the influence of including more than one (isotropic) cluster hopping parameter into the set of variational parameters. Figure 5.22 compares the staggered magnetization $m^{c}$ of the antiferromagnetic solution in the case where one hopping parameter is used to the case where all three parameters $t_{1}^{\prime}-t_{3}^{\prime}$ are used at $J / t=1.6$ as a function of electron density $n$. Off half-filling the solution with three parameters has a somewhat lower magnetization and higher critical electron density $n_{c}$. Still, comparing the energies of the respective solutions, the larger variational space leads to a lower energy at the saddle point and should be considered to be a better approximation of the system. This can be understood by looking at the values of the three different hopping parameters inside the reference cluster (see right side of figure 5.22). At first sight it might be tempting to argue, that the strong increase of the hopping parameters $t_{2}^{\prime}$ and $t_{3}^{\prime}$ leads to an increase of kinetic energy which counteracts the formation of antiferromagnetic order. Looking closer, two other differences become obvious when comparing the halffilled system with the one with reduced electron density: Firstly, the previous argument ignores the distribution of the different hopping terms inside the reference system. When averaging over the three hopping parameters $\bar{t}^{\prime}=\left(4 t_{1}^{\prime}+2 t_{2}^{\prime}+t_{3}^{\prime}\right) / 7$ is comparable to the value $t^{\prime}$ when only varying one isotropic hopping parameter. Secondly, the spreading of 

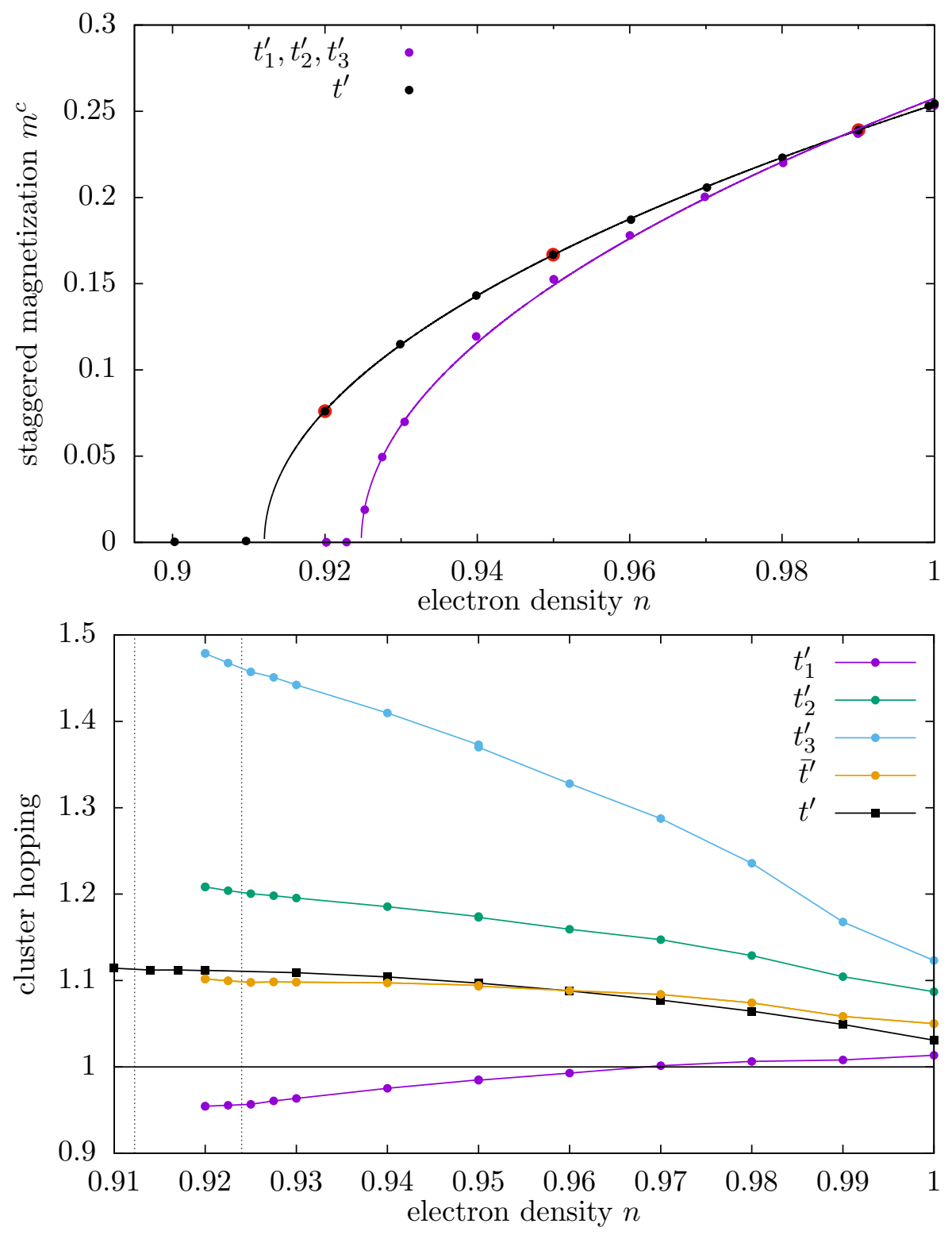

Figure 5.22: Staggered magnetization of the antiferromagnetic (AF) solution at $J / t=1.6$ as a function of electron filling $n$. In this case, using only one isotropic hopping parameter $t^{\prime}$ on the cluster as a variational parameter in contrast to three hopping parameters $t_{1}^{\prime}, t_{2}^{\prime}, t_{3}^{\prime}$ leads to a higher staggered magnetization $m^{c}$. Although the values of the cluster hopping parameters $t_{1}^{\prime}, t_{2}^{\prime}, t_{3}^{\prime}$ spread more for smaller density, the averaged hopping $\bar{t}^{\prime}$ is still comparable to $t^{\prime}$.

the three hopping parameters is much larger for small $n$ - the reference cluster simply becomes more anisotropic. Considering the antiferromagnet to be the ground state of the isotropic 2D Kondo lattice system, the latter point gives a better explanation for the decrease of $m^{c}$. As the anisotropy of the hopping parameters inside the reference system increases for a smaller electron density, it is harder to form a regularly/isotropic antiferromagnetically ordered state. In the end, the solution with three different cluster hopping parameters has a lower energy and only a bit smaller magnetization, but the qualitative results stay the same. Still, in the competition between the compensation for the edge of the cluster by adjusting the hopping parameters in an anisotropic way and 
the stabilization of antiferromagnetic order by using an isotropic staggered Weiss field the latter seems to be stronger. 


\section{Local Superconductivity in the Kondo Lattice Model}

Starting point for the study of s-wave superconductivity in the framework of a quantum cluster technique is the unexpected discovery of s-wave superconductivity by Bodensiek et al. in Ref. [BŽV $\left.{ }^{+} 13\right]$. By using DMFT with a NRG solver they identified a broad region off half-filling and for coupling strengths $J / W \gtrsim 0.1$ where the anomalous expectation value $\Phi_{s}$ had a very small but finite value. $W$ denotes the bandwidth of the bare dispersion at $J=0$, which in the square lattice amounts to $W=8 t$. A maximum of the superconducting order parameter was found at a filling of $n=0.9$ and a coupling strength of $J / W=0.3$.

Before their study, local pairing was observed already in the Kondo lattice model by meanfield approaches [HKS12, MY13], but the superconducting state found within DMFT is conceptionally different as the pairing does not occur between c- and f-electrons. Instead the superconductivity is only mediated by the antiferromagnetic spin fluctuations and pairs are formed in the conduction band only. The responsible mechanism for this pairing has already been described in section 2.3. After including temporal fluctuations within DMFT the next natural step is to examine the existence of s-wave superconductivity within a technique that includes spatial fluctuations, such as VCA.

For small clusters, VCA is known to prefer superconducting solutions even at half-filling as seen in the Hubbard model. This happens especially if the system only has a small gap as allowing for pairing to another quantum sector results in an energy gain which may be sufficient to overcome this gap. Nevertheless, VCA allowed for a qualitative study of superconductivity in the Hubbard model, which is used as a model system for hightemperature superconductors such as the cuprates.

In this chapter, the Kondo lattice model is first checked for s-wave superconductivity. At half-filling, subsection 5.3.1 shows that only using a superconducting Weiss field leads to the emergence of substantial s-wave pairing. This counterintuitive finding is subsequently shown to be an artifact of limiting the variational space too much. When including the hopping on the cluster into the set of variational parameters, the seemingly superconducting solutions turn out to be mean-field-like solutions that correspond to isolated superconducting sites. Skipping this unphysical solution, no s-wave superconductivity is found at half-filling. Afterwards the system is doped away from half-filling and the four-dimensional variational space is investigated for "new" stationary points. In subsection 5.3.2 several stationary points are discussed and it is argued that all besides the normal conducting solutions either violate thermodynamical consistency or correspond to the artificial atomic solution that was encountered at half-filling. For the $3 \times 2$ cluster a parameter regime for the Weiss field strength is identified in which stationary points could occur without ending up in those artificial solutions, but no s-wave superconducting "true" stationary points of the self-energy functional are found.

The superconducting Weiss field that amounts to s-wave singlet pairing reads as follows:

$$
\mathcal{H}_{\mathrm{SC}_{\mathrm{s}}}=D \sum_{i}\left(c_{i \uparrow} c_{i \downarrow}+c_{i \uparrow}^{\dagger} c_{i \downarrow}^{\dagger}\right)
$$


As this Weiss field does not conserve the particle number, the Hilbert space is enlarged compared to the one previously chosen, which was limited to a quantum sector with a certain electron number. The violation of particle conservation drastically increases the computational cost of the calculations which is why large $4 \times 2$ clusters are not considered in the following.

Before showing VCA results for s-wave superconductivity, it is instructive to study the effect of a superconducting Weiss field on an isolated Kondo site and on a $2 \times 2$ cluster. Results for both calculations are shown in appendix F.

\section{\begin{tabular}{l|l} 
Probing for s-Wave Superconductivity at Half-Filling & 5.3 .1
\end{tabular}}

For small coupling strengths $(J / W \lesssim 0.15)$ Bodensiek et al. surprisingly found superconductivity even at half-filling. This region differs from the rest of the superconducting phase, which is located away from half-filling and for coupling strengths $J / W \gtrsim 0.15$. However, it should be noted that the anomalous expectation value is very small in this region $\left(\Phi_{s} \approx 0.002\right)$ and the DMFT calculations suffered from convergence problems which made it difficult to stabilize the solution [Bod16]. Furthermore, this is the very region where other DMFT studies without superconducting baths found an antiferromagnetic insulator [PP07, OKK09], such that the interplay between superconductivity and antiferromagnetism should be investigated in detail.

Nevertheless, the existence of superconductivity at weak coupling within DMFT motivates taking half-filling as a starting point for the investigation of s-wave superconductivity. Figure 5.23 shows the structure of the SEF at half-filling for a $2 \times 2$ cluster. For large values of $J / t$ one finds a minimum with respect to the superconducting Weiss field strength $D_{s}$, corresponding to a superconducting solution. In addition, the SEF possesses an additional maximum for larger $D_{s}$ and yet another minimum with a larger values of the energy at weak coupling. This means, that according to Potthoff's rules for the selection of stationary points [Pot12] still the minimum with lower energy (indicated by a thick arrow in the figure) should be considered as the correct stationary point.

In contrast to the superconducting phase that was found in reference [BŽ $\left.{ }^{+} 13\right]$, the solution at the stationary point shown in figure 5.23 exists for all coupling strengths. The strength of the Weiss field at the stationary point is quite large and for coupling strengths larger than the hopping strengths $J / t \gtrsim 1$ it is even proportional to $J$. It is therefore tempting to reason that this solution amounts to a mean-field solution similar to the single-site case discussed in subsection F. This assumption is further supported by the fact that the Weiss field strength is for strong coupling independent of cluster size as shown in the right panel of figure 5.23. Extrapolating the value of $D$ to small $J$ leads to a value of $J_{0} / t=0.36$ for $3 \times 2$ clusters $\left(J_{0} / t=0.27\right.$ for $2 \times 2$ clusters $)$ where the field strength would be zero.

Compared to the atomic picture of subsection $\mathrm{F}$ the hopping term with strength $t^{\prime}=t$ enters the self-energy of the $2 \times 2$ and $3 \times 2$ clusters. As a consequence, the Weiss field strength at strong coupling is still proportional to $J$ but smaller than $3 / 4 J$. More drastic changes happen at weak coupling, where the hopping term not only contributes considerably to the ground state, but at some point changes the behavior qualitatively. 

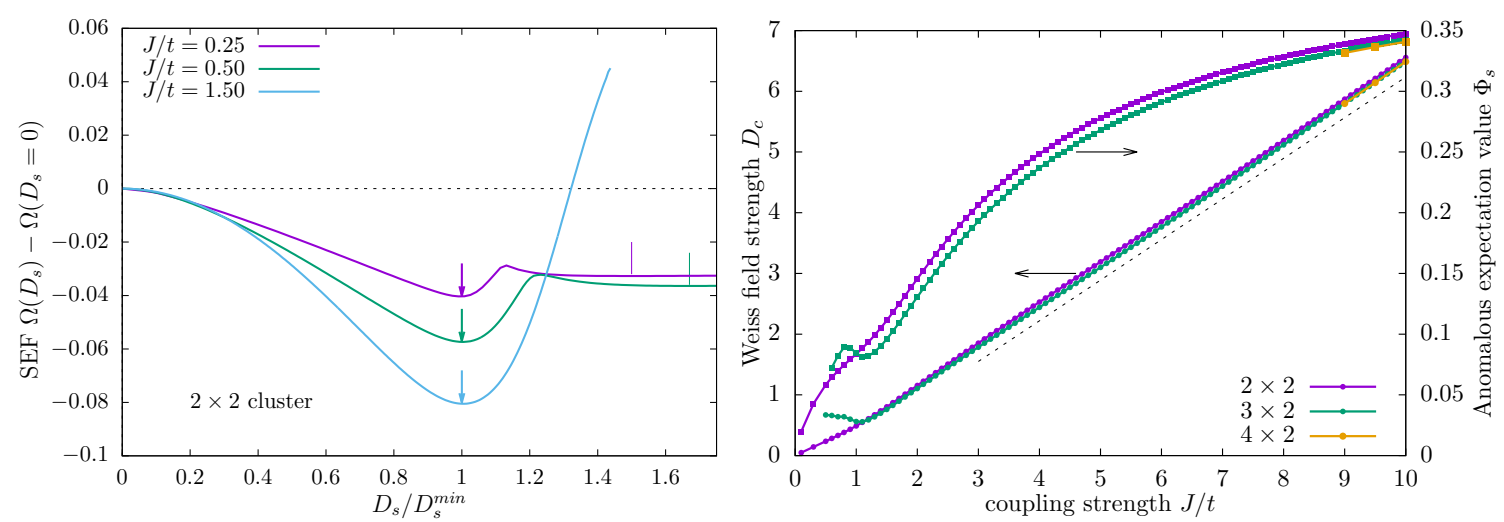

Figure 5.23: Left panel: Form of the SEF as a function of s-wave Weiss field strength for several $J / t$ using a $2 \times 2$ cluster. To compare different coupling strengths the data is plotted as a function of relative Weiss field strength, scaled by the value at the stationary point. Right panel: The value of the superconducting Weiss field $D$ and the corresponding anomalous expectation value $\langle D\rangle$ from variation of $D$ only using $2 \times 2$ and $3 \times 2$ clusters at half-filling. To show the cluster-independence of the solution at strong coupling even three values for the expensive $4 \times 2$ cluster are plotted. The dashed line is plotted for comparison to show the linear behavior in this region.

At $J / t \lesssim 1$ the behavior changes for both cluster sizes, most notably for the $3 \times 2$ cluster. Although three non-trivial stationary points (i.e. $D_{c} \neq 0$ ) exist as a function of $D_{c}$ at weak coupling, even the stationary point with the smallest $D_{c}$ exceeds the threshold of $3 / 4 J$ for a $3 \times 2$ cluster.

However, in the following section the condition $t^{\prime}=t$ will be released by including the hopping strength on the cluster into the set of variational parameters. By not fixing the hopping strength, it will be shown that the stationary point indeed corresponds to the "atomic" case considered before, even down to weak coupling.

\section{Variation of the Cluster Hopping}

The approximation within VCA is the reduction of the space of variational self-energies, which is given by the cluster geometry of the reference system and the choice of a set of one-body parameters. In most VCA studies so far, it turned out that variation of the cluster hopping terms $t^{\prime}$ did not change the results qualitatively and also did not improve them quantitatively in a significant way. However, a recent VCA study on the triangular Hubbard model showed that this variation is necessary to guarantee the stability of semi-metallic phases and that it increases the accuracy, especially for small values of $U$ [RLRT15]. It will be shown in the following that also in the case of s-wave superconductivity in the KLM, including the hopping on the cluster is crucial as it leads to qualitative changes.

Using a $3 \times 2$ cluster and varying only the s-wave pairing strength $D$ results in a seemingly superconducting solution, even for large $J / t$, with an increasing anomalous expectation value for $J / t \rightarrow \infty$, see Fig. 5.23. This is a strange result as one expects Kondo singlets to form in this limit and hence an insulator that is not superconducting. When varying $D, \mu^{\prime}$ and $t^{\prime}$ for large $J / t$ (e.g. $J / t=10$ in Fig. 5.24) and starting with the solution found previously with a large value of the pairing field, one arrives at a stationary point where 


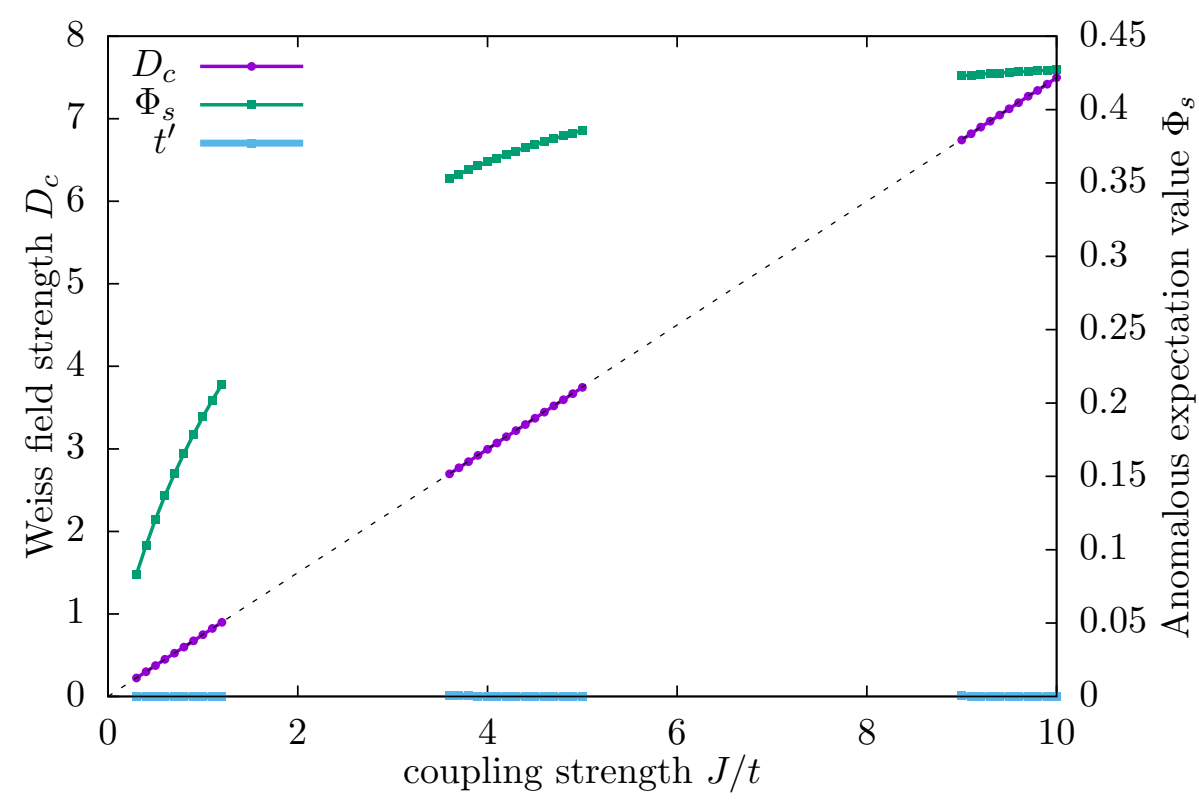

Figure 5.24: Calculation at half-filling using a $3 \times 2$ cluster, this time with the set $\left\{D, \mu^{\prime}, t^{\prime}\right\}$ of variational parameters. The hopping strength within the cluster is zero and the Weiss field strength corresponds to the one of an isolated Kondo site, $D_{c}=3 / 4 J$.

the hopping on the cluster is zero. Nevertheless, the superconducting pairing $D$ on the cluster is finite and leads to a finite anomalous expectation value at the stationary point. This corresponds to a cluster ground state with local "singlet-like" states of empty and doubly occupied sites, where single electrons are localized. For all coupling strengths $J$ the value of the Weiss field strength is given by $D_{c}=3 / 4 \cdot J$.

At half-filling the only s-wave superconducting solution is therefore the somewhat artificial superconductor that consists of local Cooper pairs without any electron hopping between the sites. A "true" superconductor comparable with the one proposed in reference $\left[\mathrm{BZZV}^{+} 13\right]$ is not found at half-filling. Next, the situation off half-filling is investigated.

\section{Choosing the Correct Stationary Point off Half-Filling}

In the next two subsections the discussion focuses on the question which of the multiple stationary points that are found off half-filling should be considered. First, the set of variational parameters is three-dimensional. In the next subsection the cluster hopping is also varied and stationary points have been determined in a four-dimensional variational space. Due to the large number of variational parameters, the $2 \times 2$ cluster will be used to find different stationary points and to discard unphysical solutions. Afterwards, the $3 \times 2$ cluster is used in subsection 5.3 .3 for calculations on the remaining solution only.

Away from half-filling, the chemical potential of the original system $\mu$ and of the reference cluster $\mu^{\prime}$ are in general non-zero. When including an s-wave superconducting Weiss field and following a stationary point, one often encounters situations where the self-energy jumps or shows a kink as a function of one of the variational parameters. Due to the presence of jumps and kinks in the self-energy functional, convergence to the correct stationary point is not ensured anymore. Sometimes, when changing a loop parameter 
(e.g. the chemical potential $\mu$ ) and starting off with the former stationary solution, one may arrive in a different quantum sector. In the self-energy functional this manifests as a jump. More generally, jumps in the self-energy functional can occur if the number of frequencies $\omega^{\prime}<0$, which enter the calculation of the self-energy functional, is changed, see formula 3.4. Changing some parameters that enter the calculation of the cluster Green function can lead to this effect. Once jumps or kinks are encountered in the SEF, the search for stationary points has to be restricted to the parameter region which causes a smooth SEF. As a consequence stationary points can be lost once they move out of this "well-behaved" region. In some of the following figures observables of the solution corresponding to this stationary point are shown, see e.g. figure 5.26. Once the stationary point is not any more in the "well-behaved" region, this amounts to a breakdown of the solution.

The task is now to choose the "correct" quantum space, which in practice amounts to choosing one combination (of at least two possible ones) of chemical potentials $\mu$ and $\mu^{\prime}$. In general, one expects to be able to tune the filling by changing the chemical potential $\mu$ around the "natural cluster fillings" $n_{\mathrm{cl}}=N_{e} / L$. In case of magnetic phases, the quantum sectors with different cluster fillings are well separated and can be connected by adapting $\mu$. In addition, one can artificially stick to a certain cluster filling, say $n_{\mathrm{cl}}=1$, and change the filling smoothly only by varying $\mu$. Here, the superconducting Weiss field couples quantum sectors with different cluster fillings, which renders the latter option impossible. In addition, even a quite weak Weiss field which couples two adjacent quantum sectors (e.g. with $N_{e}$ and $N_{e}-2$ ) could lead to an energy gain which supersedes an energy gap between these sectors. Especially for weakly gapped systems this could lead to artificial superconducting solutions due to this overcompensation effect. Overall, the addition of superconducting Weiss fields poses the problem of choosing the right stationary point, which here means to also choose out of different quantum sectors.

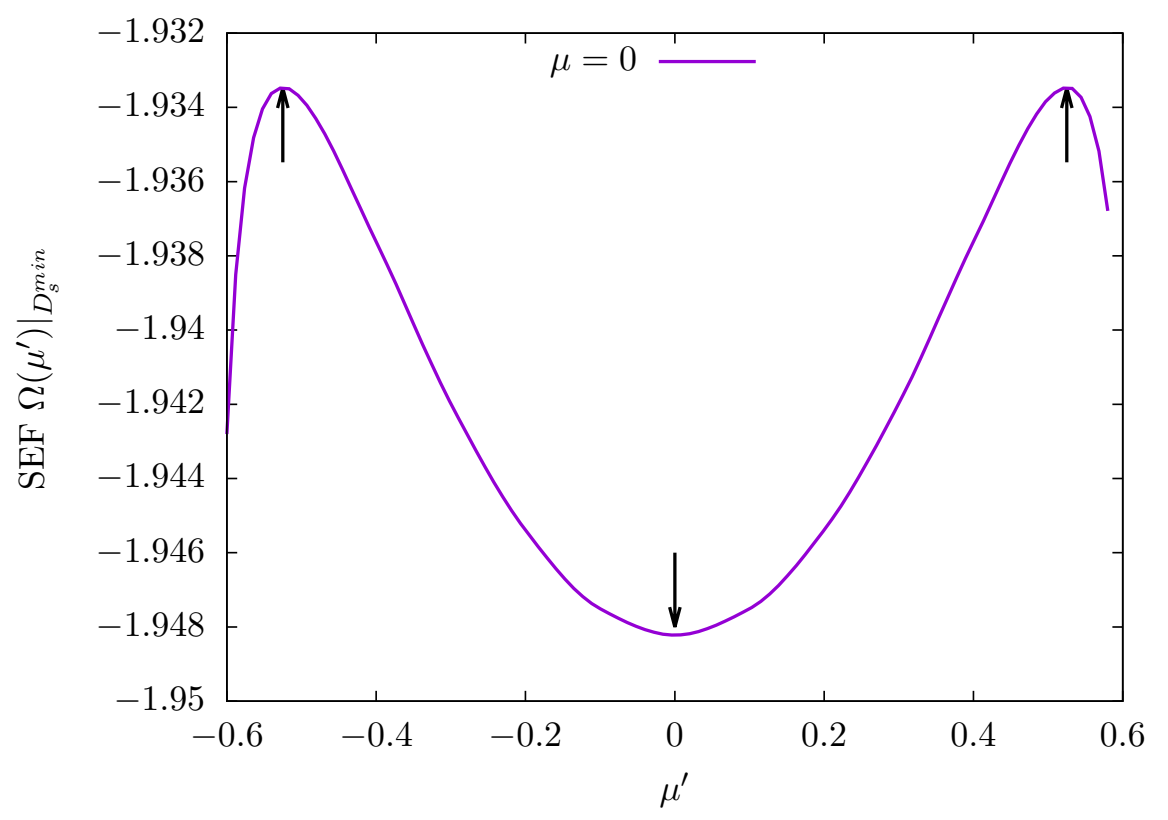

Figure 5.25: Self-energy functional as a function of $\mu^{\prime}$ for $J / t=1.5$ using a $2 \times 2$ cluster.

To illustrate this issue, a "smooth" example for the case of s-wave superconductivity using a $2 \times 2$ plaquette is discussed first. In figure 5.25 the SEF is shown as a function 
of the chemical potential of the cluster $\mu^{\prime}$ where the Weiss field strength is minimized $D_{s}=D_{s}^{\min }$ and $\mu$ is set to zero. The functional $\left.\Omega\left(\mu^{\prime}\right)\right|_{\mu=0, D_{s}^{\min }}$ is symmetric around the minimum at $\mu^{\prime \min }=0$ and possesses two additional maxima at $\mu_{ \pm}^{\prime \max }= \pm 0.525$. So far this does not pose any major problems, but it is important to note that the maxima in $\mu^{\prime}$ are close to a change in $D_{s}^{\min }$ from a finite value to zero (for $\left|\mu^{\prime}\right|>0.6$ ). At the minimum the system is half-filled $n\left(\mu^{\prime}=0\right)=1$, but for the maxima $n\left(\mu^{\prime}=-0.525\right)=1.08$ and $n\left(\mu^{\prime}=0.525\right)=0.92$. Interestingly, the anomalous expectation value $\Phi_{s}=\left\langle c_{i \uparrow} c_{i \downarrow}\right\rangle$ is non-zero for all three stationary points: $\Phi_{s}\left(\mu_{ \pm}^{\prime \max }\right)=0.077$ and $\Phi_{s}\left(\mu^{\prime \min }\right)=0.111$.

In the first instance all three stationary points are valid solutions and correspond to approximations of the true grand potential (in potentially different phases). We now trace these stationary points when slowly increasing or reducing the value of $\mu$. Unless jumps move too close to the stationary points, this procedure of "adiabatically doping" the system should lead to stationary points of the system away from half-filling.

To choose the correct solution one should compare the energy at the stationary points. Although the chemical potential $\mu$ might be the same, the number of electrons on the cluster can differ from one solution to the other as the superconducting field admixes quantum sectors with different electron numbers to the former ground state.
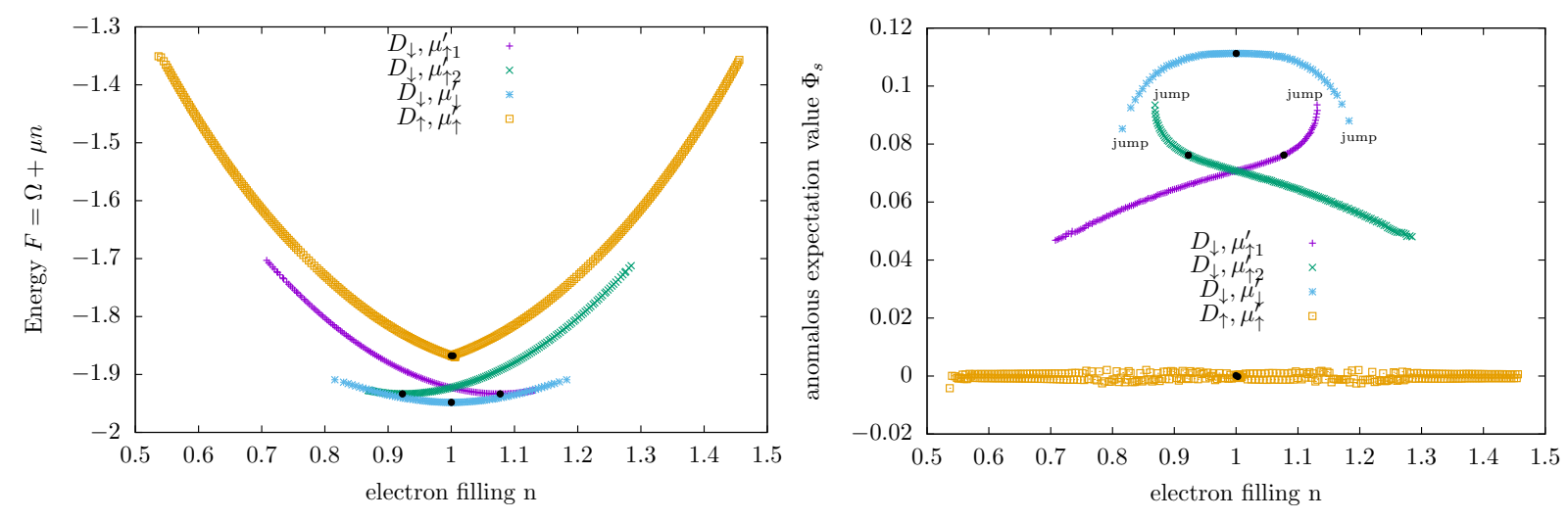

Figure 5.26: Left panel: Energy $F=\Omega+\mu \cdot n$ as a function of $n$ for different stationary points at $J / t=1.5$ using a $2 \times 2$ cluster. Right panel: Anomalous expectation value $\Phi_{s}$ as a function of $n$ for different stationary points at $J / t=1.5$ using a $2 \times 2$ cluster. The solutions for $\mu=0$ (see figure 5.25) are indicated via black dots and the two additional stationary points of figure 5.25 are denoted by $D_{\uparrow}, \mu_{\uparrow 1,2}^{\prime}$.

Figure 5.26 shows in the left panel the energies of the solutions at each stationary point. From looking only at this four stationary points, it seems that there are three different superconducting phases, which could be realized around half-filling. For strong doping, the stationary points cannot be traced any further, as jumps in the self-energy functional come too close. However, this does not mean that there is no superconducting solution available. It only shows that the superconducting solution at half-filling cannot be traced to a filling too far away from $n=1$.

The solution, which amounts to a maximum of the stationary point with respect to both $D_{c}$ and $\mu$ is the paramagnetic solution which was discussed previously as $D_{c}=0$. Comparing the other solutions, at least around half-filling the solution which amounts to a minimum with respect to $D_{c}$ and $\mu$ is the lowest in energy. Due to a discontinuity in the self-energy functional it breaks down for fillings $n \lesssim 0.82$. For $0.7 \lesssim n \lesssim 0.82$ 
the solution which is a minimum with respect to the superconducting Weiss field and a maximum with respect to $\mu\left({ }^{\prime} D_{\downarrow} \mu_{\uparrow 1}^{\prime}\right.$ ') is still lower than the paramagnetic solution.

These two stationary points will be discussed in the next subsection, where the cluster hopping will be varied in addition.

\section{Additional Variation of $t^{\prime}$}

The key question remains how the different seemingly s-wave superconducting solutions shown in figure 5.26 change if one additionally considers the intra-cluster hopping as a variational parameter. Again, one first has to search and review all the possible stationary points and then decide which ones have to be considered. This also means that there are for each solution two possible new solutions - one with $t^{\prime}$ minimizing and one with $t^{\prime}$ maximizing $\Omega$, see table 5.1. Out of eight possible combinations for stationary points, the search in the more restricted variational space of the previous subsection motivate a detailed investigation of four of them, labeled for convenience with solution 5 to 8 according to table 5.1.

\begin{tabular}{|l|c|c|c|l|}
\hline No. & $D$ & $\mu^{\prime}$ & $t^{\prime}$ & Characteristics \\
\hline \hline 5 & $\downarrow$ & $\uparrow$ & $\uparrow$ & Two solutions with $t^{\prime} \approx t$, similar to figure 5.26, \\
\hline 6 & $\downarrow$ & $\uparrow$ & $\downarrow$ & Two solutions: One has $t^{\prime}=0$, the other one is thermodynamically unstable \\
\hline 7 & $\downarrow$ & $\downarrow$ & $\uparrow$ & One solution with $t^{\prime} \approx t$, but thermodynamical stability violated off half-filling \\
\hline 8 & $\downarrow$ & $\downarrow$ & $\downarrow$ & One solution with $t^{\prime}=0$ \\
\hline
\end{tabular}

Table 5.1: Different stationary points around half-filling at $J / t=1.5$ when varying $D_{s}, \mu^{\prime}$ and $t^{\prime}$. The arrows in columns 2-4 indicate whether $\Omega$ has a maximum or a minimum with respect to the variational parameter.

When evaluating different stationary points three criteria are used to assess the solutions. One criterion is the value of the cluster hopping parameter $t^{\prime}$. "Atomic" solutions with $t^{\prime}=0$ amount to reference systems with decoupled cluster sites that locally form a superconducting singlet state. They are considered to be artificial mean-field solutions and not to represent superconductivity due to many-body effects. One of the two solutions of type 6 and the one of type 8 are therefore excluded. The remaining two types of stationary points lead to solutions with $t^{\prime} \approx t$. In the region where a smooth self-energy functional allows for the other solution of type 6 , the cluster hopping is very large $\left(1.4 \lesssim t^{\prime} / t \lesssim 2.8\right)$. Another important criterion is thermodynamical stability. At stationary points the selfenergy functional approximates the grand potential, which means that it is possible to calculate the electron density $n$ in two different ways. The filling can be obtained either by calculating the derivative of the (approximated) grand potential or by calculating the trace of the VCA Green function. In order to have thermodynamical stability, both ways of calculating $n$ should lead to the same value: $-\partial \Omega / \partial \mu=\operatorname{Tr} \mathbf{G}$. To fulfill this condition, it is usually sufficient to include the chemical potential of the cluster in the set of variational parameters. However, when checking the remaining stationary point of type 6 , both ways of calculating the electron density differ tremendously close to half-filling. The functional derivative $-\partial \Omega / \partial \mu$ does not lead to half-filling at all, such that this solution is discarded because of thermodynamic instability, see figure 5.27. In the same 

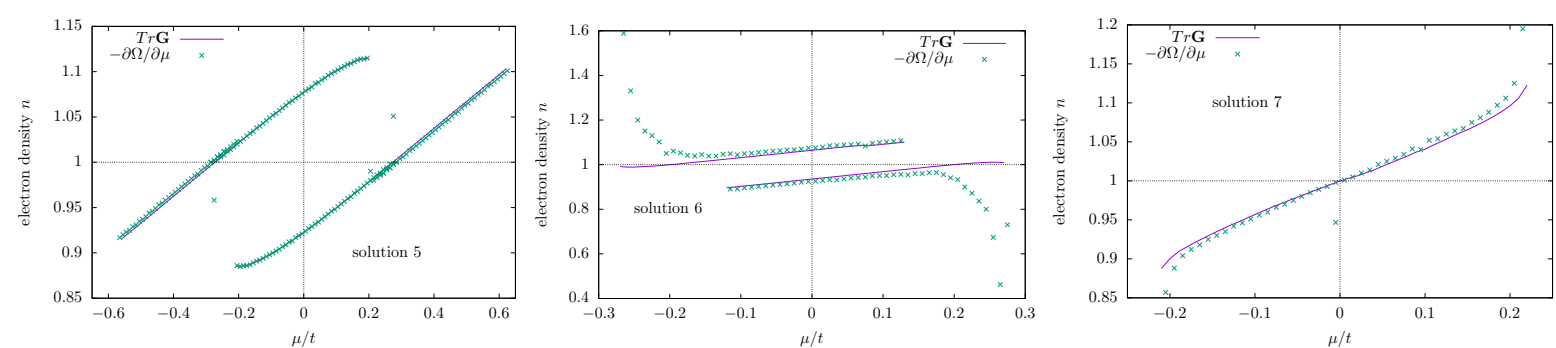

Figure 5.27: Electron density $n$ calculated as $\operatorname{Tr} \mathbf{G}$ and as $-\partial \Omega / \partial \mu$ for solutions with stationary points of type 5, 6 and 7 (from left to right) of table 5.1. In the middle panel the solution with non-zero cluster hopping $t^{\prime}$ is shown.

figure the solutions of type 5 are shown, which are thermodynamically stable. For the remaining stationary point of type $7 \operatorname{Tr} \mathbf{G}$ and $-\partial \Omega / \partial \mu$ coincide only at half-filling, but differ for all other electron fillings. Especially when approaching the breakdown region at $n \sim 0.85-0.90$ and $n \sim 1.10-1.15$ the discrepancies start to become very large.

Although this already reduces the number of solutions with $t^{\prime} \neq 0$ and thermodynamic stability to the one solution of type 5 , figure 5.28 shows solution 7 for comparison. Interestingly, all solutions break down when doping the system more than $\epsilon \sim 0.12$ away from half-filling. Most promising for further investigation away from half-filling is the solution of type 5 , that is a saddle point which is a maximum of the self-energy functional with respect to $\mu^{\prime}$ and $t^{\prime}$ and a minimum with respect to $D_{c}$. This is investigated in the next subsection.
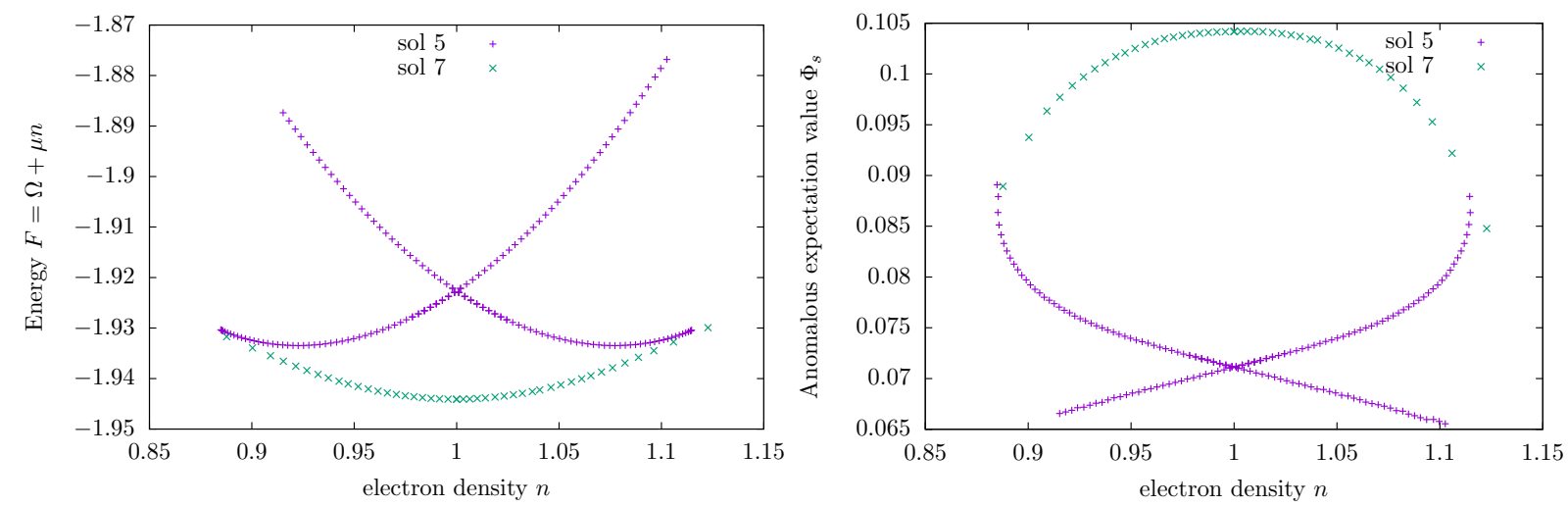

Figure 5.28: Comparison of the energies (left) and the anomalous expectation value of solutions 5 and 7 .

So far half-filling has been the starting point for the search of stationary points. In the DMFT phase diagram, superconductivity sets in for intermediate coupling strengths only away from half-filling. Therefore, the position, where the s-wave superconductivity found within DMFT was largest, is chosen as a starting point next.

\section{Absence of Local Superconductivity with a $3 \times 2$ Cluster}

Since the $2 \times 2$ cluster shows anomalies in the hopping parameter $t^{\prime}$ on the cluster already for intermediate values of $J / t$, the $3 \times 2$ cluster is considered now to investigate s-wave 
superconductivity away from half-filling. To directly compare with figures 2 and 3 of reference $\left[\mathrm{BŽV}^{+} 13\right]$ the values of $J_{1}=1.6 t=0.2 \mathrm{~W}$ and $J_{2}=2.4 t=0.3 \mathrm{~W}$ are investigated first. For the latter value, the authors of $\left[\mathrm{BZZV}^{+} 13\right]$ found the maximal anomalous expectation value $\Phi_{s}=\left\langle c_{i \uparrow} c_{i \downarrow}\right\rangle$ at a filling of $n=0.9 . J_{1}$ amounts to the value shown in their figure 3. From section 5.2.1 it is known, that within a $3 \times 2$ cluster an antiferromagnetically ordered phase is present at half-filling for $J \leq J_{c}=1.95$. Hence, we can set aside a magnetic Weiss field for the study of $J_{2}$. Doping the system results in strong suppression of antiferromagnetism, but it has to be expected, that at least close to half-filling, still both antiferromagnetic and superconducting Weiss field terms have to be considered.

One should also note, that within DMFT + NRG the antiferromagnetic phase at halffilling sets in at a critical $J_{c}^{\text {DMFT }}=0.28 \mathrm{~W}=2.24 t$ [Bod13], which means that $J_{1}$ falls into the AF phase at half-filling, but $J_{2}>J_{c}$ is not affected. In reference [OKK09] the phase diagram of the KLM at $n=0.9$ was investigated by using CT-QMC. There, the antiferromagnetic phase was found at $T=0$ for $J<J_{c} \approx 0.2 W=1.6 t$. To see antiferromagnetism for even smaller fillings one should therefore consider an even smaller J, say $J_{3}=0.15 \mathrm{~W}=1.2 t$. Although a detailed study of the interplay of AF and SC was left open in reference $\left[\mathrm{BŽV}^{+} 13\right]$, the interplay of both effects should influence the superconducting phase diagram considerably. In the low-filled case, there exist even ferromagnetic (FM) phases, as shown e.g. within the framework of DMFT[BŽPP11]. However, these FM phases appear for fillings $n<n_{c} \approx 0.65$ in the case of $J=0.2 w=1.6 t$ [BŽPP11] and are not considered in the present study.

All this considerations lead to a quite large set of variational parameters which renders the analysis of s-wave superconductivity cumbersome. A restriction of the variational space to relevant variational parameters only seems to be necessary. In the following, the hopping parameters on the $3 \times 2$ cluster will (most of the time) not be varied independently, as figure 5.21 showed no qualitative and only little quantitative differences between one and three different cluster hopping parameters in the region $1.5<J / t<J_{c} / t$. The same holds for the paramagnetic solution in the region $J_{c} / t<J / t<2.5$, so that for the investigation of $J_{1}$ and $J_{2}$ only one isotropic cluster hopping parameter in the set of variational parameters seems to be sufficient.

If one is interested in the small $J$ region, where DMFT did not find any s-wave superconductivity, this simplification is at least questionable and one should consider three hopping parameters $t_{1}^{\prime}, t_{2}^{\prime}$ and $t_{3}^{\prime}$ : At $J=0.1 W=0.8 t$ DMFT does not see any anomalous correlations for fillings $n<0.97$. In the same parameter region the deviations of the hopping parameters from $t$ are at least at half-filling very large with values up to $t_{3}^{\prime}=1.8 t$ (compare with Fig. 5.21). However, in the antiferromagnetic phase one still does not encounter an anomaly in the hopping terms as is the case for the paramagnetic solution around $J / t \approx 0.9$, see Fig. 5.2.

In contrast to the previous analysis, where no (clearly physical) s-wave solution was found, we now take the converged parameters of the paramagnetic solution at a filling of $n \approx 0.95$ as a starting point and add an s-wave superconducting Weiss field to the set of variational parameters. Again, a saddlepoint comparable to solution 6 of 5.3 .2 is found and discarded for the same reason $\left(t_{\mathrm{opt}}^{\prime}=0\right)$. Solution 5 of 5.3 .2 provided the most promising saddle point, which is why we again look for a stationary point of $\Omega$, which is a maximum with respect to $\mu^{\prime}$ and $t^{\prime}$ and which is a minimum with respect to $D$.

In figure 5.29 the cluster hopping parameter $t^{\prime}$ after optimization (solution 5) or minimization (solution 6) is plotted as a function of the superconducting Weiss field strength 
$D_{s}$. The self-energy functional as a function of $D_{s}$ is monotonically decreasing in the plotted region and only shows one stationary point, namely the maximum at $D_{s}=0$, see figure 5.29.

When comparing the values of the cluster hopping parameter $t^{\prime}$ of both solutions, one notes a change between $0.3<D_{s}<0.4$. For small Weiss field strengths the cluster hopping parameter of $\mathrm{SEF}_{5}$ starts at $t^{\prime} \approx 0.9$ and reduces when increasing $D_{s}$. At $D_{s}=0.4$ it drops to zero and remains zero for larger values of $D_{s}$. This is the somewhat artificial solution that leads at a much larger Weiss field strength to a stationary point of the SEF and at half-filling it was already shown in figure 5.24. In case of the other solution, where the value of $t^{\prime}$ leads to a maximum of the SEF, the cluster hopping is zero for small values of the Weiss field and starts to grow rapidly for $D_{s}>0.3$. For $D_{s}>0.38$, the cluster hopping parameter diverges to huge values and no stable saddlepoint, which is still a maximum with respect to $t^{\prime}$, can be identified. Comparing the values of the SEF, this amounts to the value of the Weiss field where both solutions meet, but the solution where the SEF is maximized by $t^{\prime}$ always has the lower value.

If one wants to exclude "unphysical" solutions, where the cluster consists of isolated sites and a resulting local self-energy enters the calculation of the SEF, one can restrict the search to small values of $D_{s}$. As can be seen from figure 5.29, no stationary point is found in this region $\left(D_{s}<0.4\right)$ except the non-superconducting solution at $D_{s}=0$.

The qualitative picture shown in figure 5.29 also holds at higher/lower fillings and smaller coupling strengths. For small $D_{s}$ a solution with reasonable intra-cluster hopping strength $t^{\prime} \sim t$ is found, but already for comparably small values of $D_{c} \sim 0.4-0.5$ the cluster hopping drops to zero. At this point, the cluster hopping of the second solution, which is zero for small Weiss field strengths, diverges. The solution breaks down and one is left with the case of decoupled sites inside the reference cluster that was discussed before.

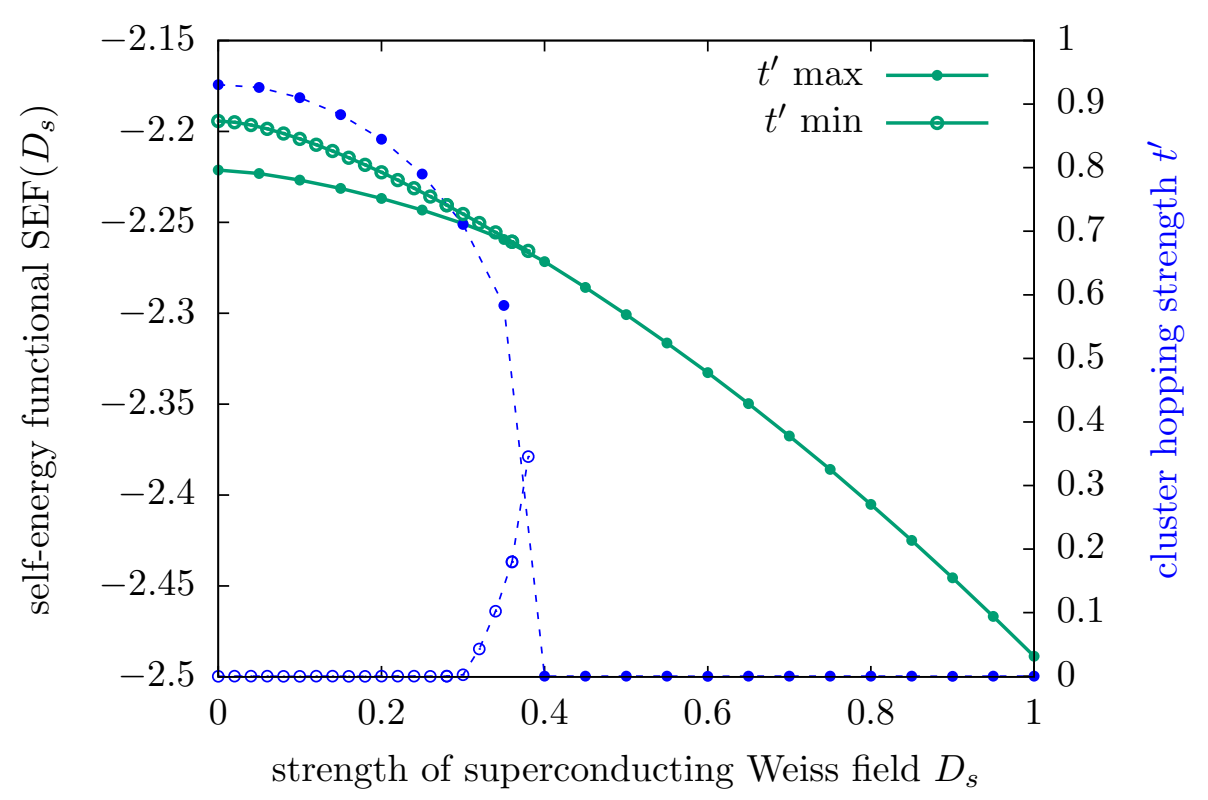

Figure 5.29: Value of the cluster hopping $t^{\prime}$ and self-energy functional $\operatorname{SEF}\left(D_{c}\right)$ at the stationary point with respect to $\mu, \mu_{c}$ and $t^{\prime}$ as a function of the Weiss field strength $D_{s}$. Plotted are two solutions for $J / t=2.4$ at $n=0.9$, one corresponding to a maximum with respect to $t^{\prime}$, the other one corresponding to a minimum. 
As no superconducting solution has been found so far, a possible next step would be to take additionally antiferromagnetism into consideration. Naturally, one might think of antiferromagnetism and superconductivity as being two competing phases. Treating antiferromagnetism and supercondictivity on the same footing would then not change the results obtained until now.

However, in both mechanisms, which were put forward by the authors of [BŽ $\left.\mathrm{B}^{+} 13\right]$ and which might explain their finding of s-wave superconductivity, spin fluctuations play an important role. When using Eliashberg theory, one of the key quantities are spin fluctuations between localized spins and conduction electrons. In subsection 5.2 it was shown that the spin-spin correlator showed qualitatively different behavior in the antiferromagnetic and in the paramagnetic phase. Although $\langle\mathbf{S s}\rangle$ should not be confused with the spin susceptibility $\chi^{\mathrm{zz}}$ that entered the Eliashberg equation, including antiferromagnetic ordering seems to be reasonable. The other mechanism proposed in [BŽ $\left.{ }^{+} 13\right]$ assumes that a local singlet can be split into a virtual triplet state before recombining at another site to a singlet again. In order to fulfill this scenario one should also assume at least a short-range antiferromagnetically ordered configuration of neighboring sites. Long-range antiferromagnetic order also renders this scenario possible.

A first indication of the effect that the addition of an antiferromagnetic to the superconducting Weiss field might have, can be gained from looking at the self-energy functional as a function of $D_{s}$ in both cases (see figure 5.30). Here, the solutions for the sets of variational parameters $\left\{\mu, \mu^{\prime}, t^{\prime}\right\}$ and $\left\{\mu, \mu^{\prime}, t^{\prime}, M_{c}\right\}$ are compared as a function of the strength of an superconducting Weiss field. Due to the outcome of the discussion in the previous paragraph, the considered Weiss field strengths are in the region of „,sufficiently small“ $D_{s}$, such that $t^{\prime} \neq 0$.

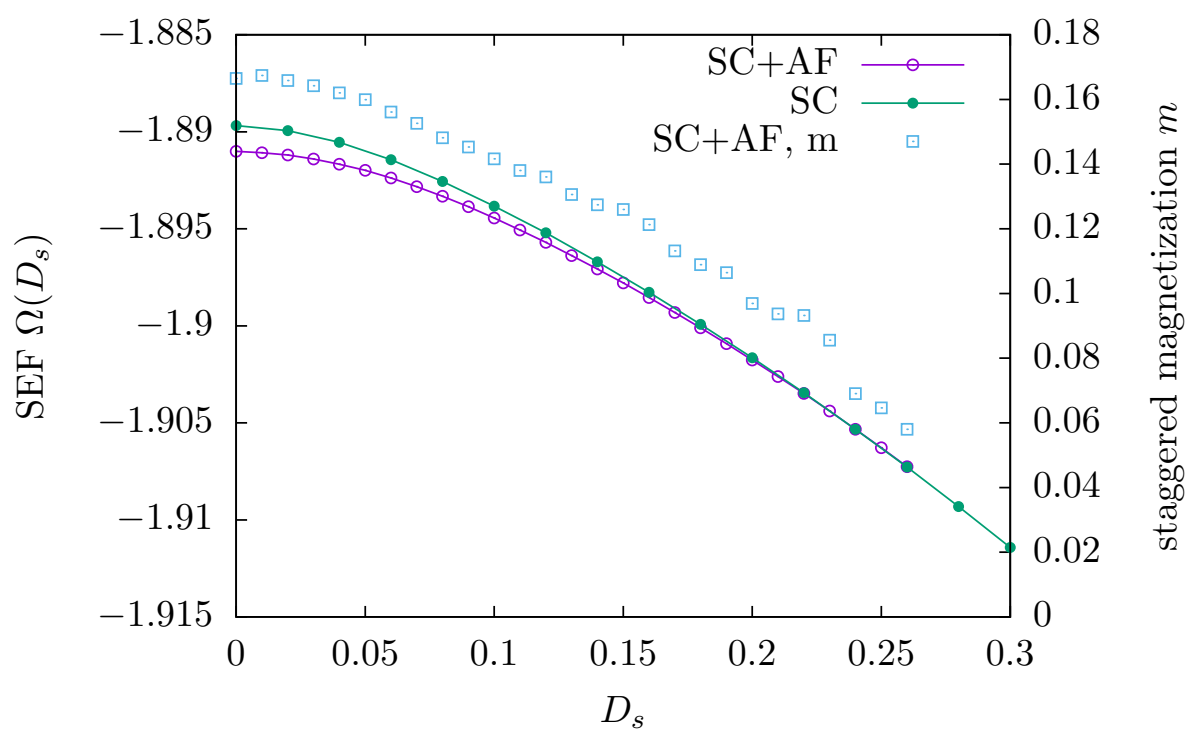

Figure 5.30: Comparison of the self-energy functional as a function of the strength of the superconducting Weiss field with ('SC+AF') and without ('SC') including $M_{c}$ into the set of variational parameters. The staggered magnetization of the ' $\mathrm{SC}+\mathrm{AF}$ ' solution diminishes for intermediate values of $D_{s}$, hence the self-energy functional approaches the 'SC' result.

The self-energy functional shows in both cases only one stationary point namely at $D_{s}=0$, which are the paramagnetic and antiferromagnetic solutions that are already known from 5.2 - the antiferromagnetic solution has a smaller energy and is therefore realized in the 
system. When increasing the strength of the superconducting Weiss field $D_{s}$ the value of the antiferromagnetic Weiss field $M_{c}$ decreases and finally goes to zero. This can be read off from the staggered magnetization in figure 5.30. The question if a superconducting solution can be realized can hence be reformulated to asking whether the change from the AF to the PM solution can be found in some parameter regime to lead to an additional stationary point.

Investigating the self-energy functional as a function of $D_{s}$ for $J=1.6 t \hat{=} 0.2 W$ and $J=$ $2.4 t \hat{=} 0.3 \mathrm{~W}$ for electron fillings down to $n=0.85$ leads to results similar to those shown in figure 5.29. This means, that even in the region where reference $\left[\mathrm{BŽV}^{+} 13\right]$ detected the largest anomalous expectation value no s-wave superconducting solutions are found. At least for these coupling strengths and close to half-filling no antiferromagnetism is found for intermediate and strong superconducting Weiss fields, which means that the search for stationary points amounts to the situation without an additional antiferromagnetic Weiss field present.

Due to the large variational space and comparatively high computational cost of the $3 \times 2$ cluster the search for s-wave superconductivity has been restricted to the region of the phase diagram which seemed to be the most promising. Based on this the existence of s-wave superconductivity in the KLM within VCA is not excluded, but in the investigated region the calculations do not show clear evidence for s-wave superconductivity. 


\section{Nodal Superconductivity in the Kondo Lattice Model}

In this section, superconductivity with different symmetry of the pairing is investigated. Especially d-wave superconductivity is often found experimentally in heavy fermion systems [SW16] and also numerical studies of the Kondo lattice model indicate the existence of a d-wave superconducting phase [AFB14, Ots15]. Although in a recent study by Otsuki a p-wave superconductor was found for coupling strengths around the critical point $J_{c}$ [Ots15], the focus of this section will be on superconductivity with $d_{x^{2}-y^{2}}$ symmetry.

First, the paramagnetic solution will be taken as a starting point to investigate superconductivity by adding a Weiss field with d-wave symmetry. In subsection 5.4.2 antiferromagnetism will be treated on equal footing with superconductivity and the interplay of both symmetry broken phases will be discussed.

\section{\begin{tabular}{l|l} 
d-Wave Superconductivity & 5.4 .1
\end{tabular}}

In case of s-wave superconductivity the Cooper pairs form locally and clusters are affected uniformly by the Weiss field. The geometry and size of the cluster enter the calculation through the intra-cluster hopping and in case of antiferromagnetism through the mediated effective RKKY interaction only. Considering extended pairing, such as the non-local $d_{x^{2}-y^{2}}$ superconductivity, this changes. Due to its geometry, the $2 \times 2$ cluster is for instance known to favor $d$-wave pairing. For this reason the $2 \times 2$ cluster will only be briefly discussed and mainly used as a reference to the $3 \times 2$ cluster, for which most of the results will be shown.

As long as no antiferromagnetic Weiss field is used in addition to the superconducting one, the paramagnetic phase diagram at half-filling has to be used as a starting point. In contrast to the "full" phase diagram, which includes antiferromagnetism and leads to an insulator at arbitrary coupling strength $J / t$ at half-filling, the paramagnetic phase diagram also shows a metallic phase. To be more precise, the system is metallic in the weak coupling region and becomes insulating when the coupling exceeds some value $J>J_{c \text {,INS }}$, where Kondo screening is big enough to form an insulator consisting of the Kondo singlets.

When looking at the anomalous expectation value $\Phi_{d_{x^{2}-y^{2}}}$ which serves as the order parameter of a superconducting phase it is not surprising, that no superconductivity is found in the insulating region, see figure 5.31. In contrast to the expectation that there should not be any superconductivity at all at half-filling, one finds d-wave superconductivity for weak coupling up to $J / t \approx 1.1$. This is the region where the incomplete (paramagnetic) treatment of the system showed an anomaly in the cluster hopping. For the $2 \times 2$ cluster the cluster hopping has a minimum at $J / t \sim 1.4$ and starts to grow for smaller coupling; in case of the $3 \times 2$ and $4 \times 2$ cluster $t^{\prime}$ even showed a kink when plotted as a function of $J$ at $J / t \sim 0.8$, which marked the phase transition to a metal for smaller coupling strengths. As will be shown below, the onset of superconductivity for the $3 \times 2$ cluster is comparable to this coupling strength $J_{c \text {,INS }}$. Although this reveals the need of including antiferromagnetism into the calculations, it still provides the correct starting point for the strong coupling region, i.e. the paramagnetic region $J>J_{c, \mathrm{AF}}$. There, leaving half-filling should be valid as antiferromagnetism is not realized at or off half-filling as was shown in 

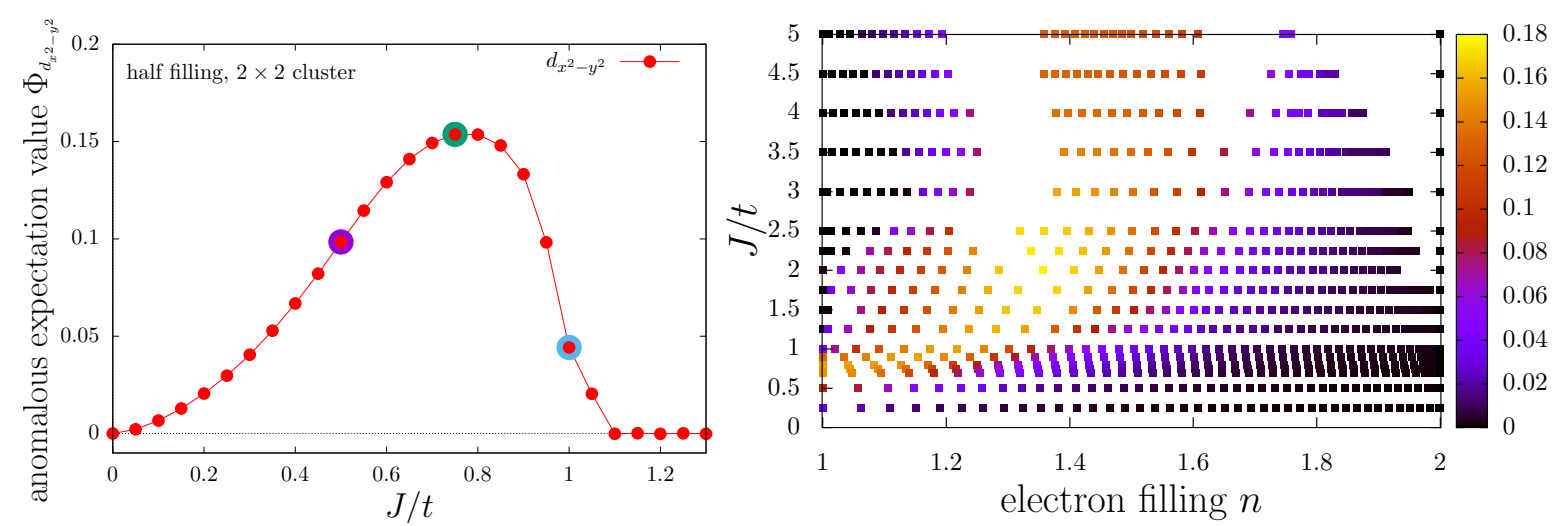

Figure 5.31: Anomalous expectation value $\Phi_{d_{x^{2}-y^{2}}}$ as a funciton of $J / t$ at half-filling (left) and as a function of $n$ and $J / t$ (right). Calculations have been done using a $2 \times 2$ cluster, ignoring possible magnetic solutions.

section 5.2 .

Leaving the study of the region with coupling $J<J_{c} \approx 2.05 t$ to the next subsection where antiferromagnetism is included in the investigation, it remains to discuss the region with $J>J_{c}$. Still, it is interesting that the maximum of the anomalous expectation value $(J / t \sim 2.1)$ amounts to the region where antiferromagnetic fluctuations lead to the onset of AF long-range order if one permits this ordering. The corresponding electron density at the maximum is roughly $n \approx 0.65$. Close to half-filling the paramagnetic metal persists at coupling stengths $J>J_{c}$ and only doping of $\epsilon \sim 0.1-0.2$ leads to a finite $\Phi_{d}$. When lowering the electron density further, the size of the anomalous expectation value diminishes and finally goes to zero at small $n$.

Before including an antiferromagnetic Weiss field, d-wave superconductivity is investigated in the region around $J_{c}$ using a $3 \times 2$ cluster, see figure 5.32. For this cluster $J_{c} / t \approx 1.95$. The overall phase diagram compares qualitatively well to the one of the $2 \times 2$ cluster and it even gives quantitatively similar results.

\section{\begin{tabular}{l|l} 
Competition of Antiferromagnetism and Superconductivity & 5.4 .2
\end{tabular}}

In the previous subsection we have seen, that away from half-filling superconducting phases can manifest in the phase diagram of the Kondo lattice model. Especially for small couplings $J$ another symmetry breaking enters in the form of antiferromagnetic ordering of the conduction electrons. The interplay of these two effects is known to be important for d-wave superconductivity in the Hubbard model as possibly realized in high-temperature superconductors. In principle there are three scenarios which are possible. The most improbable situation is the one where the two phases are independent and hence, considering magnetism and superconductivity leads to the same magnetization and anomalous expectation value as treating these effects separately. It is also possible that both phases coexist and that they either compete, which means that the onset of antiferromagnetism reduces superconductivity and vice versa, or that they cooperate. In the latter case, the superconductivity would be enhanced due to the antiferromagnetic 


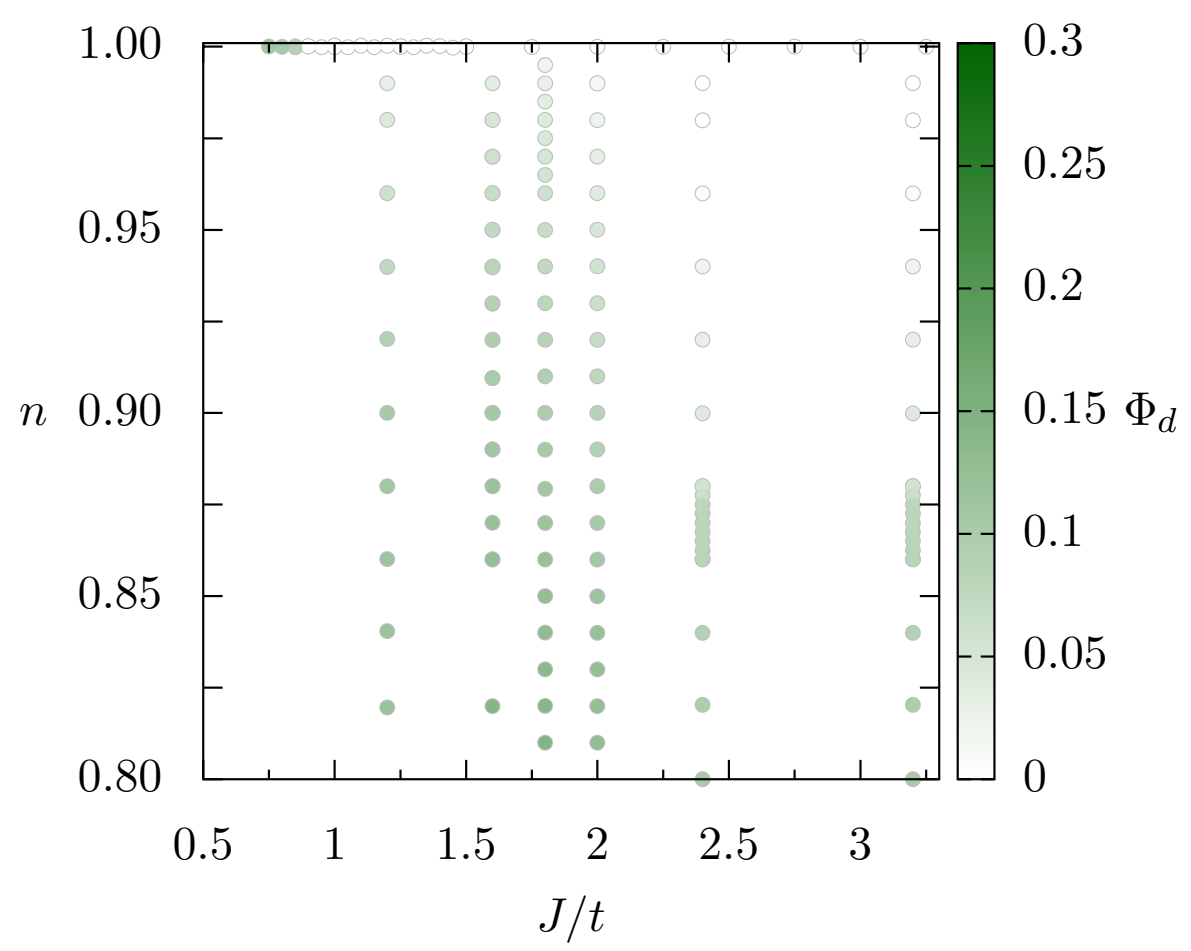

Figure 5.32: Anomalous expectation value $\Phi_{D}$ using a $3 \times 2$ cluster and variation parameters $\mu^{\prime}, t^{\prime}$ and $D$. For $J \leq J_{\text {ano }}^{S C} \approx 0.9$ there is a superconducting solution even at half-filling, but one has to be careful as this region coincides with an anomaly in $t^{\prime}$. The interaction strength $J$ where the anomaly occurs is quite close to the one found in the paramagnetic solution $\left(J_{\text {ano }}^{P M} \approx 0.84\right)$.

ordering.

However, considering the results of the previous section, approaching this question within VCA might seem to be tricky as one encountered problems for weak coupling. Nevertheless, as this is the very region where at least in the normal phase antiferromagnetism dominates one has to consider both broken symmetries together to properly address this weak to intermediate coupling regime. As shown in chapter 5.2 antiferromagnetism already sets in for intermediate interaction strengths where the divergence of $t^{\prime}$ does not yet pose a problem, but one has to bear in mind that any doping of the system reduces the antiferromagnetic correlations. Hence, doping the system sufficiently in order to observe superconductivity might already be too much doping to observe antiferromagnetism. Especially for intermediate coupling strengths close to $J_{c}^{\mathrm{AF}}$ this means, that one has to investigate a very narrow $\mu$ window corresponding to small doping.

In figure 5.33 a $3 \times 2$ cluster is used to revisit the half-filled system at $J<J_{c}^{\mathrm{AF}}$, this time using both a d-wave superconducting and an antiferromagnetic Weiss field at the same time. For all coupling strengths the solution coincides with the antiferromagnetic insulator that was already found in section 5.2. Of course the stationary point with $M=0$ still exists, but a comparison of the corresponding energies shows that the antiferromagnetically ordered phase is always lower in energy. Especially at weak coupling, allowing for both, superconductivity and antiferromagnetism, results in an antiferromagnetic insulator and no superconducting solution with lower energy is found at half-filling.

In case of the $3 \times 2$ cluster the critical coupling strength $J_{c, A F} \approx 1.95$, hence the value of $J / t=1.8$, which is used for figure 5.34, corresponds to the region of the antiferromagnetic 


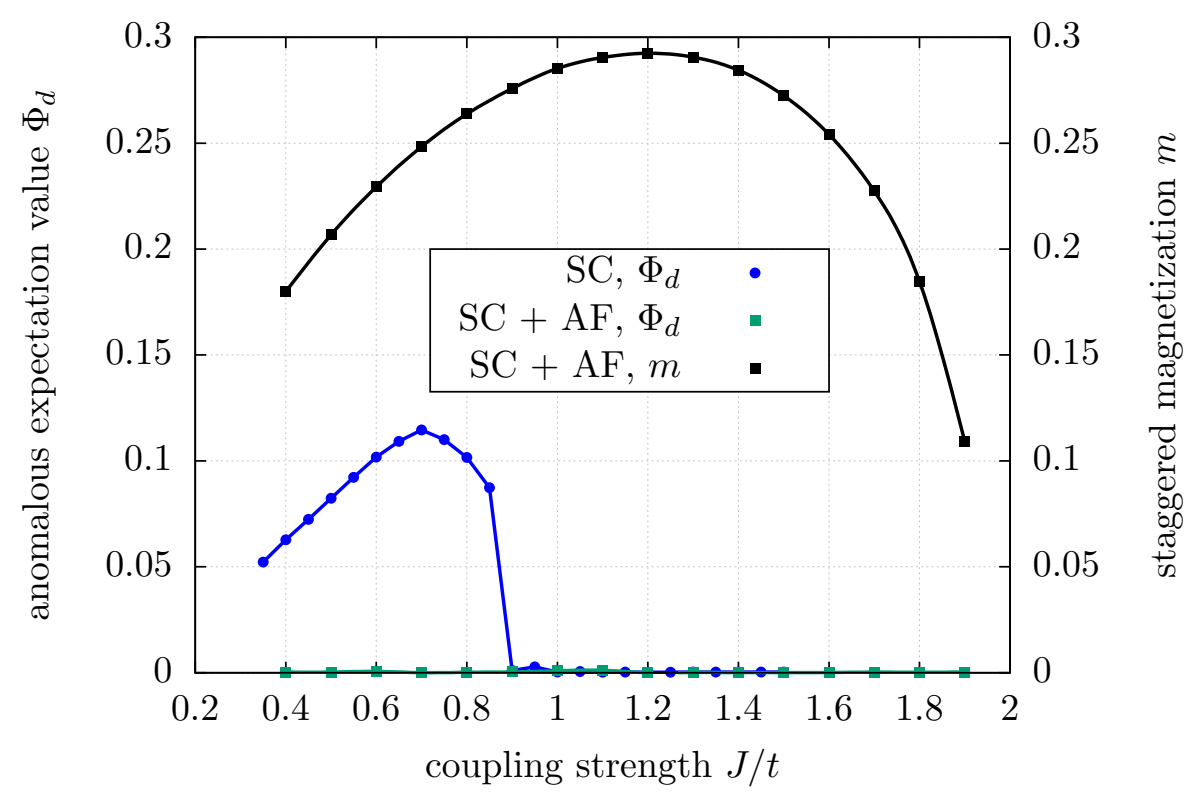

Figure 5.33: Stationary points of the self-energy functional using only a superconducting Weiss field and using in addition an antiferromagnetic Weiss field on a $3 \times 2$ cluster. When allowing for antiferromagnetism, no d-wave superconducting solution is found at half-filling.
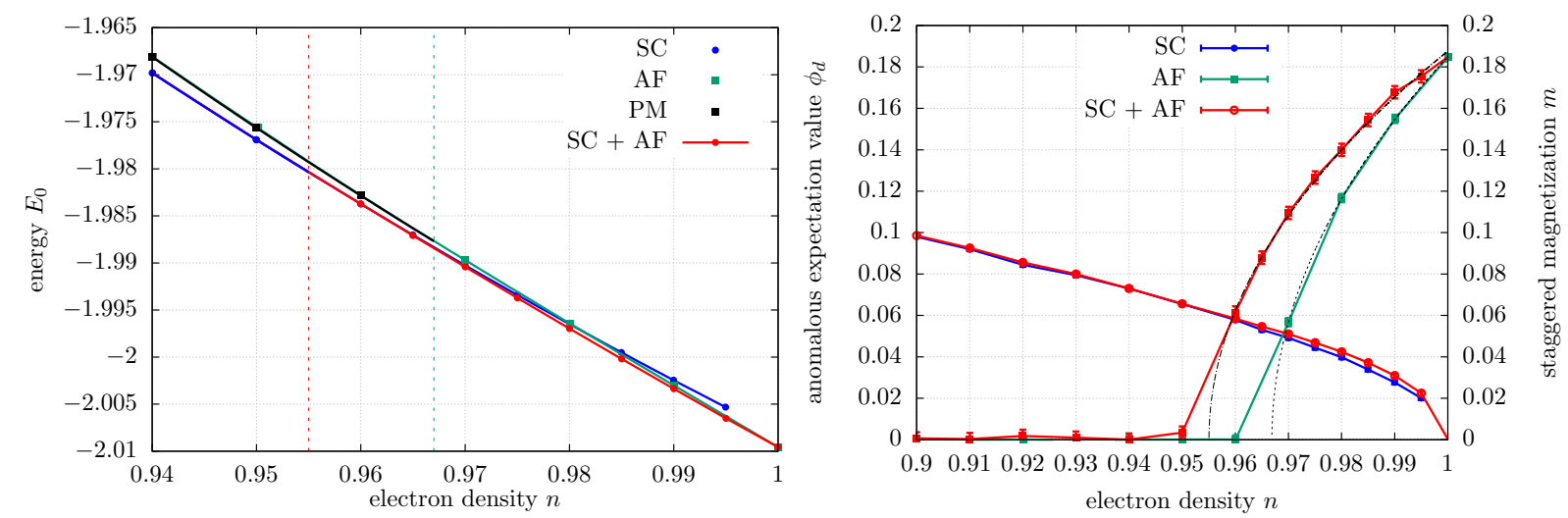

Figure 5.34: Using a $3 \times 2$ cluster at $J / t=1.8$, the system is doped away from half-filling. The left panel shows the energy density and the right panel the staggered magnetization and anomalous expectation value as a function of electron density $n$ for the superconducting, the antiferromagnetic and the coexisting $\mathrm{SC}+\mathrm{AF}$ solutions.

metal with large Fermi surface down to $n_{c}$, where it turns into a paramagnetic metal. The other value shown in figure 5.35 is $J / t=1.2$ and corresponds to the antiferromagnetic metal with a small Fermi surface. This intermediate coupling strengths are chosen such that one avoids the Lifshitz transition from large to small Fermi surface inside the antiferromagnetic metal phase.

Figure 5.34 shows both the pure antiferromagnetic and the pure d-wave-superconducting solutions as well as a solution with coexistence of superconductivity and antiferromagnetism. Starting with the "pure" solutions at $J / t=1.8$, for small doping the antiferromagnetic solution has a lower energy than the superconducting one, but their energies 
cross at $n \approx 0.98$. From considering these two phases only, the system would be antiferromagnetic for $0.98 \leq n \leq 1$ and d-wave superconducting for fillings smaller than 0.98 . However, compared to the "pure" phases, the solution with coexistence of AF and SC has the lowest energy and should therefore be realized in the system.

In the coexistence region the anomalous expectation value does not change much compared to the solely superconducting solution with $M_{c}=0$. At the same time the staggered magnetization is enhanced compared to the AF solution. The coexistence region therefore enlarges the superconducting region to a value close to half-filling and extends the antiferromagnetic region down to an electron density of $n \approx 0.955$. In this sense, the interplay of antiferromagnetism and d-wave superconductivity at $J / t=1.8$ can be considered to be cooperative. Outside of the coexistence region, no antiferromagnetic order is present and the solution coincides with the one shown in figure 5.32 where d-wave superconductivity without antiferromagnetism was considered.
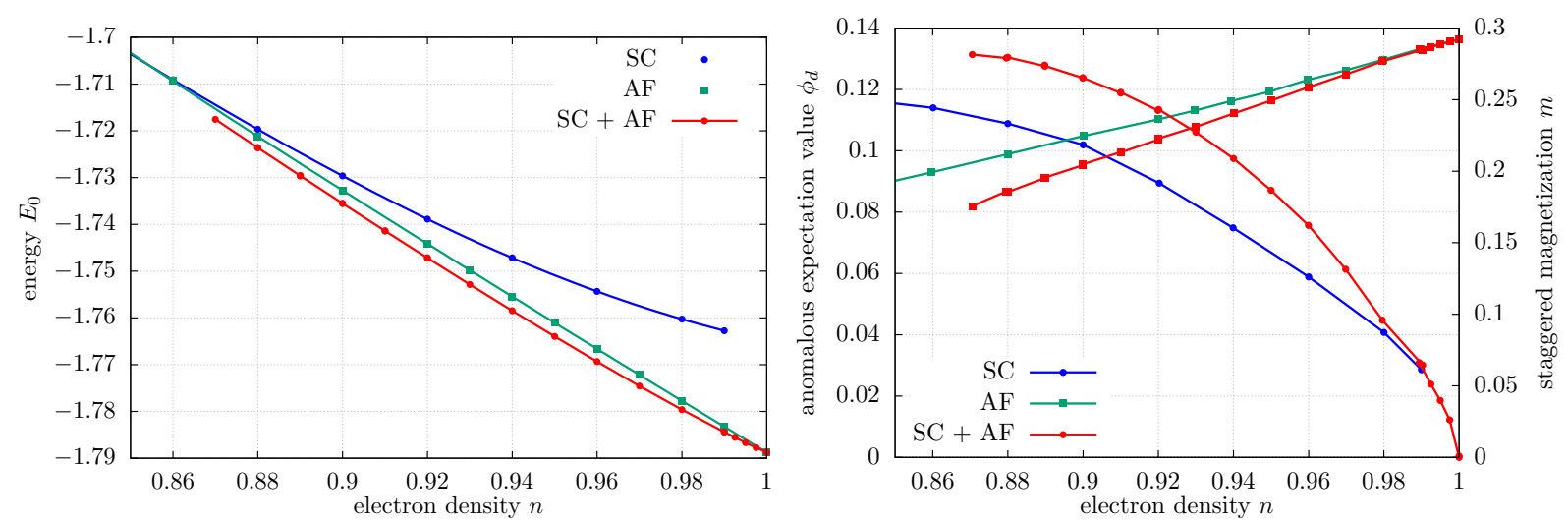

Figure 5.35: The same plots as in figure 5.34 showing the energy (left panel) and the order parameters (right panel), this time for $J / t=1.2$.

When reducing the coupling strength, the interplay between antiferromagnetism and superconductivity changes. Figure 5.35 shows the same quantities for a coupling of $J / t=1.2$. Still, there exists a coexisting region which has the lowest energy and which is therefore preferred as compared to the pure AF and SC solutions. Close to half-filling $(n \gtrsim 0.98)$ the coexistent solution has comparable order parameters as the pure solutions. For smaller electron density the staggered magnetization of this solution is reduced compared to the pure antiferromagnetic phase, but the anomalous expectation value is perceptibly larger than the one of the pure d-wave solution. When extrapolating the staggered magnetization it becomes clear, that the antiferromagnetic region will be reduced compared to the antiferromagnetic phase diagram shown in figure 5.15. At the electron density $n_{c}$ where the antiferromagnetic order breaks down the pure superconducting solution should be recovered. Until then (i.e. for $n_{c}<n<0.98$ ) the anomalous expectation value is larger than in the pure d-wave solution.

The interplay of both symmetry-breaking mechanisms is therefore characterized by a competition between superconductivity and antiferromagnetism at $J / t=1.2$.

The results of the investigation of the interplay of antiferromagnetism and d-wave superconductivity are summarized in figure 5.36. It shows the coexistence region of an antiferromagnet and a superconductor. This region is limited to an electron density of $n=1$, where superconductivity breaks down, and by a critical coupling strength $J_{c}(n)$ 


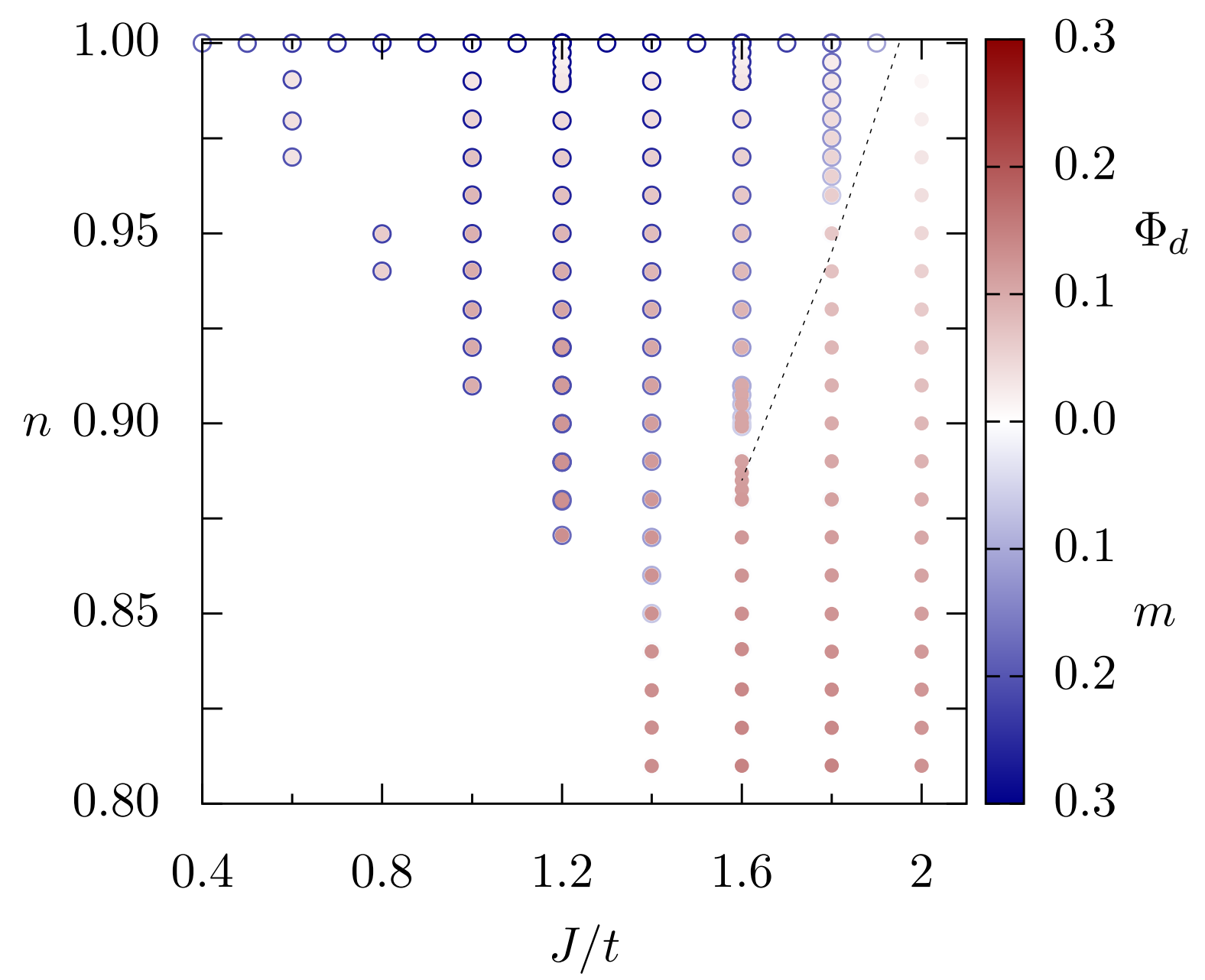

Figure 5.36: Anomalous expectation value (intensity color-coded in red) and staggered magnetization (in blue) using a $3 \times 2$ cluster allowing for both superconductivity and antiferromagnetism.

(indicated by a dashed line in figure 5.36), which marks the breakdown of the antiferromagnet. 


\section{\begin{tabular}{l|l} 
Summary & 5.5
\end{tabular}}

In this chapter the variational cluster approximation was used to investigate the Kondo lattice model directly at zero temperature. Exploiting the strength of VCA that phases with broken symmetry can either be probed or actively avoided by choosing a suitable variational space, the different phases of the KLM were analyzed separately. Afterwards the interplay of these phases was investigated and finally combined to a phase diagram that includes all different phases. As the half-filled KLM poses a special point in the phase diagram, it has been analyzed separately from the doped model. In each part of the analysis the quality of the VCA approximation has been checked by choosing different technique-specific parameters such as the cluster size and the set of variational parameters.

The examination of the Kondo lattice model progressed in three parts. First, the paramagnetic phase of the model was treated and built the foundation for the inclusion of symmetry-breaking mechanisms. In part two the antiferromagnet at small to intermediate coupling strengths was discussed and the last part focused on superconductivity of sand d-wave symmetry.

In section 5.1 the paramagnetic phase was studied first at and afterwards off half-filling. Due to the absence of Weiss fields the set of variational parameters is comparatively small such that it was possible to investigate cluster size effects and to identify a necessary minimal set of variational parameters. In the process differences between the $2 \times 2$ and the $3 \times 2$ and $4 \times 2$ clusters were found at small coupling and the influence of multiple (anisotropic) hopping strengths for asymmetric clusters turned out not to change the results qualitatively. At half-filling all clusters lead to a Kondo insulator with a nearly cluster size independent quasiparticle gap at strong coupling. The asymmetric $3 \times 2$ and $4 \times 2$ clusters showed an unexpected transition to a paramagnetic metal at $J / t \approx 0.8$. As one expects for this region long-range antiferromagnetic order due to effective RKKY interaction, the metallic phase at half-filling has not been investigated in detail. Doping the system away from half-filling at strong coupling resulted in a metallic phase with a large Fermi surface. The Fermi surface area in this region amounts to the sum of electron density and f-spin density, which indicates the participation of $\mathrm{f}$-spins in the charge transfer process via mobile Kondo singlets.

Allowing for symmetry breaking in the spin-channel via a suitably chosen Weiss field in section 5.2 made the investigation of an emerging antiferromagnetic phase below a critical coupling strength $J_{c}$ possible. At half-filling this value was determined for several cluster sizes and geometries such that a rough finite-size extrapolation revealed an infinite-clustersize value of $J_{c}^{\mathrm{VCA}} \approx 1.48 \pm 0.28$. Although only three cluster sizes could be used, the extrapolated value already nicely fits to the exact result of a QMC calculation by Assaad [Ass99]. Here, improved cluster solvers, which make use of cluster symmetries, or even solvers based on DMRG in an efficient implementation using matrix product states could perhaps allow for larger cluster sizes and allow for a more precise finite-size extrapolation. The transition from a paramagnetic to an antiferromagnetic insulator was analyzed in detail and qualitative differences as well as characteristic features were identified in the spin-spin correlator and the density of states. Off half-filling the antiferromagnetic solution becomes metallic and the staggered magnetization is lowered when reducing the 
electron filling. At some critical electron filling $n_{c}(J)$ the magnetization vanishes smoothly and the system continuously goes over to a paramagnetic metal.

A detailed study of the antiferromagnetic metal revealed two different regions. One region at weak coupling with a small Fermi surface and another region at larger coupling with closed pocket structures. By revisiting the spin-spin correlator and the structure of the density of states (DoS) as a function of coupling strength at half-filling and analyzing the evolution of the DoS as a function of electron density, the influence of Kondo singlets in the antiferromagnetic phase was investigated. Although no definite proof could be given, the analysis suggested the existence of Kondo singlets in the antiferromagnetic phase down to weak coupling. This would contrast the Kondo breakdown scenario of mean-field theory [ABF13]. However, a discontinuous transition between the two phases with different Fermi surface topology away from half-filling was found. This is consistent with results obtained with variational Monte Carlo [WO07, ABF13], but opposes the scenario of a continuous Lifshitz transition, which is supported by RDMFT [PK15] and DCA [MA08, MBA10].

As the variational cluster approximation used in this chapter is limited to the electronic degrees of freedom and does not include excitations of the f-spins in the Green function, the question of Kondo screening is difficult to answer. Including spin excitations in an extension of standard VCA might allow for a detailed investigation of Kondo screening so that the question of a possible Kondo breakdown could be readdressed.

Section 5.3 focused on the question whether s-wave superconductivity is realized in the Kondo lattice model as found within $\mathrm{DMFT}+\mathrm{NRG}\left[\mathrm{BŽV}^{+} 13\right]$. Already at half-filling using the $2 \times 2$ cluster a seemingly superconducting solution could be traced back to a solution of atomic mean-field nature. By extending the variational space, additional stationary points of the self-energy functional were investigated and most of them were discarded for two reasons. Some of the points corresponded to the artificial mean-field solution of isolated superconducting sites, others violated thermodynamic stability. To exclude artificial solutions, a parameter regime for the Weiss field strength was identified and investigated for the most promising stationary points using the $3 \times 2$ cluster. In this parameter regime of small Weiss fields the only stable solutions corresponded to a paramagnetic metal and not to an s-wave superconductor. Also allowing for a possible coexistence with antiferromagnetism did not lead to stable s-wave superconductivity either.

Moving to superconductivity of d-wave symmetry, section 5.4.1 first showed the emergence of stable d-wave superconducting solutions for weak coupling at half-filling. This region could be related to the metallic region of the paramagnetic phase in case of the $3 \times 2$ cluster. Away from half-filling the paramagnetic metal at strong coupling continuously develops d-wave pairing and turns into a superconductor.

Including antiferromagnetism in addition destroyed superconductivity at half-filling and lead to a pure antiferromagnetic insulating phase instead. For coupling strengths $J<J_{c}$ a coexistence region was identified away from half-filling down to a critical density $n_{c}(J)$. Inside this coexistence phase two regions were found, one displaying competition between antiferromagnetism and superconductivity (small coupling) and one region where both mechanisms act cooperatively (close to $J_{c}$ ).

The results motivate further studies on the Kondo lattice model. Two routes can be pursued in future investigations. 
On the one hand, the VCA results considering the first-order transition in the antiferromagnetic phase and the absence of local superconductivity ask for further comparison with other numerical methods. Besides improving the VCA results by treating larger clusters, perhaps using other cluster solvers like DMRG, the clusters could be extended by adding bath sites. Thereby, the changes due to the inclusion of temporal fluctuations can be monitored. In this way, some of the discrepancies between techniques like VMC, which treat finite lattices, and embedding techniques like DMFT-based methods might be clarified.

On the other hand, the Kondo lattice model could be extended to connect closer to experiments. Especially adding terms that compete with Kondo-singlet formation, such as additional long-range hopping terms, to the Kondo lattice Hamiltonian is promising. In this way the question of a (possibly) tunable Kondo breakdown might be approached via VCA. The consequences of such additional terms on superconductivity are also interesting. A route which is often pursued in this context consists in adding non-local Heisenberg interactions between the f-spins. However, this would require further developments of the variational cluster approximation to include such non-local spin interactions. First steps in this direction have been done already by Filor and Pruschke [FP14]. 


\section{\begin{tabular}{l|l} 
& \\
Conclusions & 6
\end{tabular}}

In this thesis different aspects of unconventional phases in strongly correlated electron systems were analyzed. Paradigmatic models for two specific classes of these systems have been investigated numerically by using different variants of the variational cluster approximation (VCA). On the one hand, the Hubbard model, which is a paradigmatic model for the Mott metal-insulator transition (MIT), has been analyzed. On the other hand, the phase diagram of the Kondo lattice model (KLM), which is an effective model for heavy fermion systems, has been investigated.

In a digression a spin version of a precursor technique of VCA, spin cluster perturbation theory (SCPT) has been benchmarked and an idea from standard cluster perturbation theory transferred to SCPT. This bridges a gap between SCPT for strongly dimerized chains [OBS10] and spin variational cluster approximation [FP10, FP14].

In both model systems studied in this thesis, quantum critical points were found. For the anisotropic two-dimensional Hubbard model, the MIT was found to become continuous at a critical anisotropy strength in the paramagnetic case. By further increasing this anisotropy parameter into the quasi-one-dimensional region of the model, the transition stayed continuous. Preliminary studies on the antiferromagnetic phase also gave indications for this quantum critical line. The universality class of the MIT and, especially in experiments, putative Mott quantum criticality is heavily debated. For both problems the finding of this quantum critical line opens new possibilities for future research. In contrast to this quantum critical line, the existence of a quantum critical point in the KLM is well-established, but its nature is still discussed in context of a putative Kondo breakdown. Here, this issue is readdressed using the variational cluster approximation for the first time directly on this model. The KLM on a two-dimensional square lattice showed an antiferromagnetic phase at weak coupling up to a quantum critical point. Inside this antiferromagnetic phase two regions were identified, which are separated by a first-order transition and which differ in their Fermi-surface topology. The model was also probed for s-wave superconductivity, but only non-local d-wave pairing was found to be robust. Its interplay with the antiferromagnetism at weak coupling was investigated and different qualitative behavior was observed. Both the first-order transition in the antiferromagnetic metal and its interplay with d-wave superconductivity ask for further studies that allow for better comparison with other numerical approaches and experimental studies.

Controlled numerical approaches to two-dimensional models for strongly correlated electron systems are rare or only applicable in a certain parameter regime [LAB $\left.{ }^{+} 15\right]$. Two techniques that belong to the most accurate of these numerical methods are large-scale cluster simulations via quantum Monte Carlo (QMC) [APMH94, Ass99, LAB ${ }^{+}$15] or via tensor networks such as projected entangled-pair states (PEPS) [VC04, COBV10, Orú14, Cor16]. However, they both suffer from problems which render these calculations computationally costly. In case of QMC this is the fermionic sign problem, in case of PEPS the area law for entanglement. 
In VCA exact diagonalization is used as a cluster solver, which circumvents these problems, but limits the reference systems to small clusters. The VCA is a suitable tool to study phases with broken symmetry in the Hubbard and Kondo lattice model in two dimensions. In some sense, the technique works directly in the thermodynamic limit, but the finite size of the reference cluster influences the approximation. The quality of the approximation is improved by increasing the size of the cluster, which allows to control the technique. Besides the small cluster sizes, another drawback of VCA is its mean-fieldlike character when it comes to correlations that exceed the cluster size. Nevertheless, VCA has shown promising results for one- and two-dimensional model systems, see, e.g., [BHP08, BP10, $\mathrm{DAH}^{+}$04, $\mathrm{BKS}^{+}$09].

Hence, despite their limitations, by using variants of the variational cluster approximation, important aspects could be added to the discussion of Mott quantum critical behavior and to the discussion of the phase diagram of the KLM.

In the following two sections, perspectives and possible further investigations that emerge from the results of chapters 4 and 5 are laid out. The results obtained therein are summarized briefly, a more detailed summary can be found in sections 4.4 and 5.5, respectively.

\section{Mott Criticality in the Anisotropic 2D Hubbard Model}

In case of organic charge-transfer salts, which show a Mott metal-insulator transition when tuning the pressure experimentally, the nature of the endpoint of the metal-insulator transition line and the corresponding universality class are still heavily discussed [KMK05, $\left.\mathrm{AJKW}^{+} 15\right]$.

By studying the Mott metal-insulator transition (MIT) in an anisotropic two-dimensional Hubbard model a quantum critical point at $T=0$ is identified, which continues as a quantum critical line to the quasi-one-dimensional regime of the model. In combination with complementary cellular DMFT (CDMFT) calculations done by M. Raczkowski, a consistent picture of the MIT at low to zero temperature could be obtained. Further results for the antiferromagnetic phase suggest a similar picture and motivate to add an anisotropy parameter as a new axis in the phase diagram. This adds a new route to explain the low temperature critical endpoint of systems like layered organic charge-transfer salts, which can be modeled by anisotropic two-dimensional Hubbard models. Furthermore, the quantum critical line encountered in this thesis motivates to further study the universality of the MIT as it might allow to explain some fingerprints of the unconventional critical behavior in these compounds.

Introducing a new axis to the known phase diagram in form of a parameter that controls the anisotropy of the system thereby offers the possibility to tune the critical temperature to zero. Following up on this new way of tuning the critical temperature motivates further studies of the Mott metal-insulator transition. The quantum critical behavior emerging from the quantum critical point adds a new aspect to the discussion of universality of the MIT and might allow to explain at least part of the unconventional critical behavior in layered organic compounds in future studies. There are various possible routes which could be pursued. In the following I will sketch three of them which seem very promising 
to me.

One route relates to the critical hopping strength $t_{\perp}^{c}$, where the MIT turns from a continuous to a discontinuous transition. It is discussed whether this point could be a marginal quantum critical point (MQCP), which was introduced in the context of metal-insulator transitions by Imada, see [Ima04, Ima05]. In his works, the analysis of MQCPs leads to different scaling laws depending on the phase from which the point was approached. Particularly, critical exponents were determined that fit within error bars to those of the unconventional universality class observed in experiments by the group of Kanoda [KMK05]. However, the analysis of the MQCP was limited to the frustrated two-dimensional Hubbard model on square and triangular lattices using Hartree-Fock theory. Studying the putative MQCP with the methods used in this thesis that go beyond Hartree Fock is therefore a promising approach and might lead to better understanding of the universality of the MIT.

The results of this thesis from VCA at zero temperature combined with finite-temperature CDMFT results, as presented in reference $\left[\mathrm{LMP}^{+} 16\right]$, motivates further studies using finite-temperature VCA. Within quantum Monte Carlo the fermionic sign problem renders smaller temperatures than those shown in $\left[\mathrm{LMP}^{+} 16\right]$ barely accessible. Using VCA at extremely low temperatures $0 \leq T \leq 0.03$ is therefore desirable to access this important region of the phase diagram. It is possible to evaluate the self-energy functional numerically at non-zero temperatures, but the calculation of the finite-temperature cluster Green function needs to be efficient. Two promising candidates are advancements on finite-temperature Lanczos methods [JP94, ADEvdL03] and using the density-matrix renormalization group (DMRG) technique at finite temperatures, e.g. within an efficient matrix product state formulation [TMPH14], to calculate the cluster Green function. By using DMRG it might also be possible to treat larger clusters at zero temperature than currently possible with the Band-Lanczos algorithm.

When connecting the new findings of this thesis to experiments on organic charge-transfer salts, it should be noted that the $\kappa$-phase of organic charge-transfer salts is often described with Hubbard models on triangular lattices. Although these models include an anisotropic hopping, the geometry of the lattice prevents a clear separation of frustration and dimensionality as it has been done in this thesis. However, it is also important to bare in mind that the triangular geometries used in these models are the result of assuming dimerization of neighboring molecules in a more general model. This simplification was often simply taken for granted and is well justified for a lot of compounds. Still, in a recent study using density functional theory in combination with random phase approximation effective lattice models with four different hopping parameters were derived for selected superconducting $\kappa$ - $(\mathrm{ET})_{2} \mathrm{X}$ materials [GAJV16]. It turned out that the assumption of dimerization cannot be justified for these cases and a more complicated lattice has to be considered to describe properties like the symmetry of the emerging superconducting Cooper pairs correctly. Hence, extending the present study to a more general model, e.g. using the derived model of reference [GAJV16] instead of the triangular lattice, seems promising. 


\section{Superconductivity and Antiferromagnetism in the Kondo Lattice Model}

For certain heavy fermion systems the low-energy physics is well described by the Kondo lattice model [Don77, Col07]. In a recent study [BŽV $\left.{ }^{+} 13\right]$, s-wave superconductivity was reported for this model within a dynamical mean-field theory. In this thesis, the variational cluster approximation was for the first time applied directly to the Kondo lattice model to check the system for s-wave superconductivity with a technique that incorporates non-local correlations. Although a VCA study found (different) s-wave pairing in the periodic Anderson model [MY15], the type of superconductivity proposed in [BŽ $\left.{ }^{+} 13\right]$ was not found for the KLM in this thesis. Nevertheless, here other phases with broken symmetries have been studied in the Kondo lattice model, too.

In the paramagnetic phase of the KLM, the Kondo insulator at half-filling and the metallic phase with heavy charge carriers away from half-filling have been investigated. By including an antiferromagnetic Weiss field, the antiferromagnetic phase was then analyzed. For weak coupling up to a quantum critical point at coupling strength $J_{c}$, the system was found to order antiferromagnetically. Two antiferromagnetic phases with different Fermi-surface structure separated by a first-order transition were identified away from half-filling. This finding agrees with variational Monte Carlo results [WO07, ABF13], but disagrees with result of techniques based on DMFT [MA08, MBA10, HK13, PK15], where the transition was found to be continuous. By using a suitable Weiss field, the Kondo lattice model was probed for local s-wave superconductivity, which, as mentioned above, was reported recently using DMFT with an NRG solver [BŽ $\left.{ }^{+} 13\right]$. After artificial superconducting solutions at and away from half-filling were sorted out, no local superconductivity due to correlation effects between the electrons was seen via VCA. Instead, robust d-wave superconductivity was found for a large range of coupling strengths and electron fillings away from half-filling. Its interplay with antiferromagnetism at weak coupling showed qualitatively different behavior in the two different antiferromagnetic regions. Thereby a full phase diagram of the Kondo lattice model was presented in this thesis via variational cluster approximation including paramagnetic, antiferromagnetic and d-wave superconducting phases.

The VCA studies in this thesis have shown the capabilities of this quantum cluster method to investigate phases with broken symmetry directly in the Kondo lattice model. This opens different routes, which could be pursued in future studies. In the following I will sketch three in my opinion promising continuations.

To follow up on the question of the nature of the local pairing mechanism identified in $\left[\mathrm{BŽV}^{+} 13\right]$, future studies should include temporal fluctuations, too. Techniques like dynamical impurity approximation and variational cluster approximation with additional bath sites are the next logical candidates that could help to bridge the gap from the perspective of quantum cluster techniques. In this way the present study could be compared to a related technique which includes only temporal fluctuations (DIA), and one that in addition to (short-range) spatial fluctuations also includes temporal fluctuations. As a recent study using the dual fermion technique did not find local superconductivity in the KLM [Ots15], it is also possible that the emergence of s-wave superconductivity in $\mathrm{DMFT}+\mathrm{NRG}$ is more due to the way superconductivity is included in the formalism pro- 
posed in $\left[\mathrm{BŽV}^{+} 13\right.$, Bod16]. Nevertheless, a systematic study of local superconductivity with additional quantum cluster techniques might help to clarify this issue.

The antiferromagnetic phase off half-filling turned out to be divided into two regions with different Fermi surfaces which are separated by a discontinuous transition. This discontinuous transition was also obtained in studies using variational Monte Carlo techniques [WO07, ABF13]. Numerical techniques, which include temporal fluctuations to bath sites like dynamical cluster approximation [MA08, MBA10] or real space dynamical mean-field theory [PK15] found a continuous Lifshitz transition between these two phases. To illuminate this discrepancy, it could be again useful to study the Kondo lattice model via VCA with additional bath sites. However, coming back to superconductivity, within VMC indications of d-wave superconducting correlations were found, too, but a stable superconducting phase only existed when spoiling antiferromagnetism, e.g. by adding frustration [ABF13, AFB14]. In this thesis, the d-wave superconducting phase was robust and even coexisted with antiferromagnetism at weak coupling. However, one has to keep in mind that quantum cluster techniques like VCA often tend to overestimate d-wave superconductivity on the square lattice. Nevertheless, the discrepancy between both techniques asks for further investigation. One possibility to clarify this would consist in doing a proper finite-size scaling of the VCA data to see whether the d-wave superconductivity survives this extrapolation or whether it is a finite-size effect. Unfortunately, due to the large Hilbert space dimension and the kind of solver used to calculate the cluster Green function the calculations are limited to small clusters. This renders a finite-size scaling at the moment not possible. Calculating the Green function with other techniques like DMRG as already proposed in subsection 6.1 could show a way out.

Finally, it should be pointed out that extensions of the Kondo lattice model might allow to connect closer with experiments on heavy fermion systems. Especially if one wants to address the question whether a local quantum critical point (involving a Kondo breakdown) can be found in the Kondo lattice model, this could be necessary. Adding terms that lead to a destruction of Kondo singlets which then results in an (eventually) tunable Kondo breakdown is at least one possibility to approach this question. Frustration in form of additional long-range hopping terms is a promising route that has been used in the past, see, e.g., [MBA10, AFB14]. Another approach, which is often done to investigate d-wave superconductivity, consists in including additional (non-local) Heisenberg interactions between the f-spins. This leads to variants of the so-called Heisenberg Kondo lattice model; for numerical studies see, e.g., [XD08, AFB14, YGML14]. However, as these non-local spin interactions are not easily compatible with standard variational cluster approximation, as a cluster tiling cuts inter-cluster interaction terms, further developments on VCA, perhaps in the direction of spin VCA proposed by Filor and Pruschke [FP10, FP14], would be necessary. Recently, Steglich and Wirth discussed the link between a Kondo-destroying (local) QCP and an orbital-selective Mott transition in the heavy fermion system $\mathrm{YbRh}_{2} \mathrm{Si}_{2}$ [SW16]. Therefore, it might also be interesting to resort to the periodic Anderson model and to compare with the results of the KLM. There, the VCA was already successfully applied and s-wave superconductivity between c- and f-electrons was found [MY15]. A comparison of this (different) type of superconductivity with the superconductivity found in the KLM is also still an open issue. 
To conclude, there are many possible routes for future research directions using variational cluster approaches. Both from a theoretical and an experimental point of view, the new aspects that result from the investigation of unconventional phenomena in this thesis motivate further investigations. Some of the presented perspectives involve an adaptation of the model systems studied here, others require further technical developments. All of them can build on the results of this thesis and represent possible exciting future studies. 
Appendices 



\section{Calculation of the Green Function in Continued Fraction Representation via the Lanczos Algorithm}

Instead of using the Band Lanczos algorithm to evaluate the Green function $\mathbf{G}$ using the $\mathbf{Q}$-matrix notation, the standard Lanczos algorithm can be used to evaluate $\mathbf{G}$ in a continued fraction representation. In the end one has to calculate the action of $(\omega+$ $\left.H-E_{0}\right)^{-1}$ on the excited state $\left|\phi_{\mu}\right\rangle=c_{\mu}^{\dagger}|\Omega\rangle$. The advantage of working in the Krylov subspace can be seen when expanding the fraction in powers of $\mathrm{H}$ :

$$
\frac{1}{\omega-H+E_{0}}=\frac{1}{\omega-E_{0}}+\frac{1}{\left(\omega-E_{0}\right)^{2}} H+\frac{1}{\left(\omega-E_{0}\right)^{3}} H^{2}+\ldots
$$

Applying this to a normalized starting vector $\left|\phi_{0}\right\rangle=\left|\phi_{\mu}\right\rangle / \sqrt{\left\langle\phi_{\mu} \mid \phi_{\mu}\right\rangle}$ thus gives vectors of the Krylov space. Using the Lanczos algorithm with this starting vector leads to coefficients $\left\{a_{n}\right\},\left\{b_{n}\right\}$ and a tridiagonal representation of the Hamiltonian. After $b_{n}$ has reached a value less than some threshold value $\epsilon_{\text {tol }}$, the Lanczos recursion is stopped and the Green function is calculated. To be more precise the diagonal element $G_{\mu \mu}(\omega)=$ $\left\langle\phi_{\mu} \mid \phi_{\mu}\right\rangle\left[\left(\omega-H+E_{0}\right)^{-1}\right]_{00}$ is calculated as a continued fraction [Dag94]:

$$
G_{\mu \mu}(\omega)=\frac{b_{0}^{2}}{\omega-a_{0}-\frac{b_{1}^{2}}{\omega-a_{1}-\frac{b_{2}^{2}}{\omega-a_{2}-\ldots}}} .
$$

Off-diagonal entries of the Green function can be evaluated by constructing the combination of two creation/annihilation operators:

$$
G_{\mu \nu, e}(\omega)=\frac{1}{2}\left(G_{\mu \nu, e}^{+}(\omega)-G_{\mu \mu, e}^{\prime}(\omega)-G_{\nu \nu, e}^{\prime}(\omega)\right)
$$

with

$$
G_{\mu \nu, e}^{+}(\omega)=\left\langle\Omega\left|\left(c_{\mu}+c_{\nu}\right) \frac{1}{\omega-H+E_{0}}\left(c_{\mu}^{\dagger}+c_{\nu}^{\dagger}\right)\right| \Omega\right\rangle .
$$

As long as small and intermediate clusters are considered, the computational cost for calculating the Green function in the Lehmann representation within the Band Lanczos algorithm is still acceptable. For large clusters the larger memory needs of the Band Lanczos algorithm might be problematic and one might want to resort to the calculation of $\mathbf{G}$ in a continued fraction representation via the Lanczos algorithm. In the latter case, orthogonality leaks may show up in the spectral function and canceling these leaks by brute-force Gram-Schmidt reorthogonalization or a selective reorthogonalization [Ruh00] might become necessary. However, these reorthogonalization schemes also lead to larger computation times, which is why orthogonality leaks, which are not too severe, are often not corrected in practice [Sén12]. 



\section{Additional Spin Structure Factors for Heisenberg Models}

First, additional results for the spin structure factor of the isotropic Heisenberg model are shown using spin cluster perturbation theory. Then, the spectra for the anisotropic XXZ chain are shown and briefly discussed. Afterwards the $J_{1^{-}} J_{2}$ Heisenberg ladder is investigated. The latter is interesting as on the one side it causes more inter-cluster interactions, but on the other side the frustration in the model should reduce long-range correlations.

\section{\begin{tabular}{l|l} 
Isotropic Heisenberg chain & B.1
\end{tabular}}

In the following, additional results for the isotropic1D Heisenberg model are shown. For the plots of the spectral function the $k$-space has been discretized equidistantly with $N=200 \mathrm{k}$-points and a artificial broadening of $\eta=0.02$ is used.
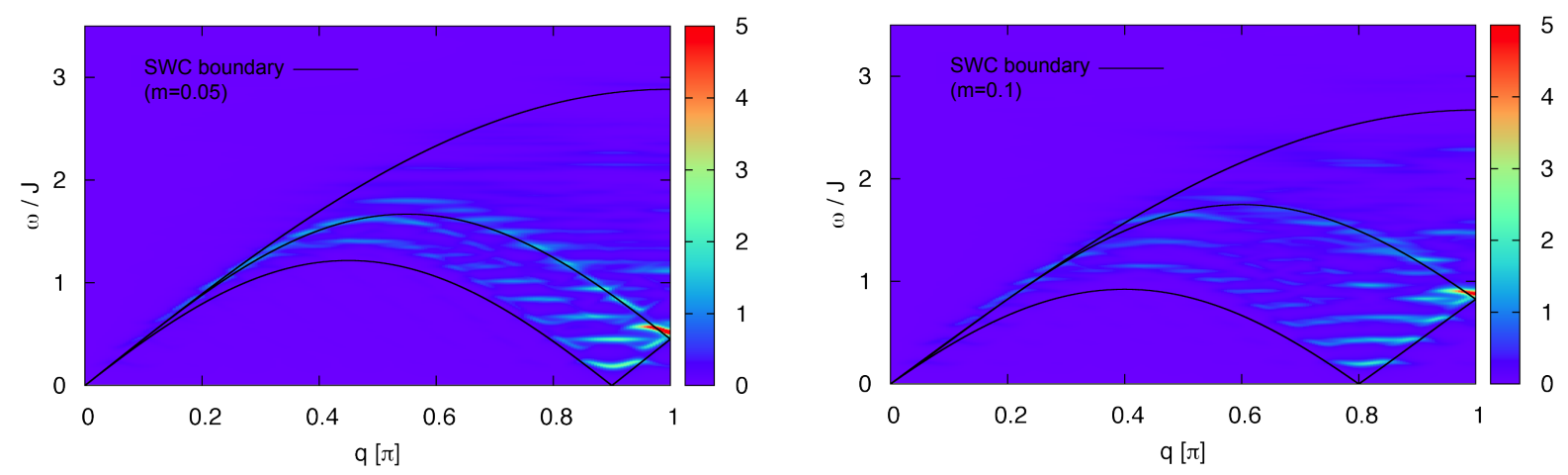

Figure B.1: Spin structure factor $S^{z z}(q, \omega)$ using a 20-site cluster. The external magnetic field $h / J$ corresponds to magnetizations of $m=0.05$ (left panel) and $m=0.1$. For comparison, the limits of the spin wave continua contributing to $S^{z z}$ of an infinite Heisenberg chain are shown as obtained in reference [MTBB81].

Figure B.1 shows the spin structure factor $S^{z z}(q, \omega)$ of the isotropic Heisenberg chain for two different magnetizations.

The boundaries in figure B.1 follow the spin wave continua derived by Müller et al. in reference [MTBB81]. For zero magnetic field only one spin wave continuum (SWC) exists and is bounded by $\epsilon_{1 L}(q)=2 J \sin \frac{q}{2} \cos \left(\frac{q}{2}-\pi m\right)$ and $\epsilon_{1 U}(q)=2 J \sin \frac{q}{2}$, where $m$ denotes the magnetization (here zero) and the wave numbers $q$ have to fulfill $2 \pi m \leq q \leq \pi$. The total spin of this excitations is $S=L_{c} m+1$ and the z-component $S_{z}=L_{c} m$. At zero magnetic field this is the dominant contribution to the spin structure factor, but for finite magnetic field it becomes negligible in the limit $L_{c} \rightarrow \infty$. For finite magnetic field, a second spin wave continuum with total spin $s=L_{c} m$ exists. It is limited by $\epsilon_{2 L}(q)=2 J\left|\sin \frac{q}{2} \cos \left(\frac{q}{2}+\pi m\right)\right|$ and $\epsilon_{2 U}(q)=\epsilon_{1 L}(q)$ for $0 \leq q \leq \pi$. When treating increasingly larger spin chains, the contribution of the first spin wave continuum diminishes 
and it is the second spin wave continuum that dominates. These limits are shown for comparison in figures B.1.

For higher values of the magnetization $m$ deviations of the lowest excitations with considerable weight and the lower boundary of SWC becomes apparent. The fact that the lower boundary is not reached between $0<q<q_{0}$ is a phenomenon which has been encountered before in ED and is typical for finite-size chains [dCP62, MTBB81]. In fact as the first SWC with higher excitations is negligible in the limit $L_{c} \rightarrow \infty$, one would expect that the spectrum lies with the boundaries of the second SWC when the cluster size becomes large. For infinitely large clusters one expects the first SWC to become negligibly small. Therefore the spectrum shifts towards the boundary of the second SWC when increasing the cluster size.

\section{\begin{tabular}{l|l}
$X X Z$ Heisenberg Chain & B.2
\end{tabular}}

Adding an anisotropy in $z$-direction leads to the XXZ Heisenberg chain with $\Delta \neq J$.
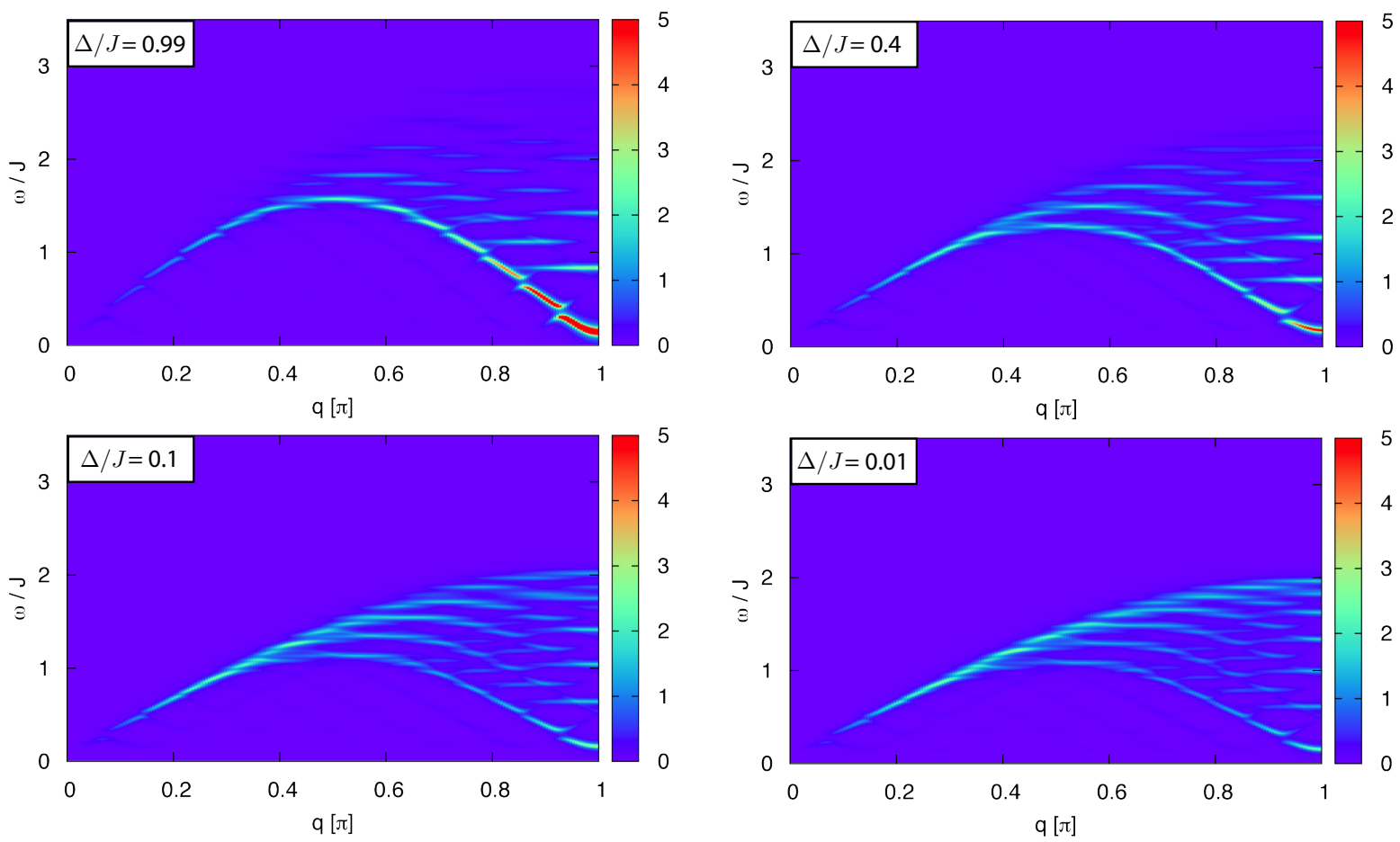

Figure B.2: Spin structure factor $S^{z z}$ of the XXZ Heisenberg chain with different anisotropy strengths (from top left to bottom right: $\Delta / J=0.99,0.4,0.1,0.01$ ) using spin CPT with a cluster of size $L_{c}=14$.

Results for the spin structure factor $S^{z z}$ can be seen in figure B.2, where the XXZ Heisenberg chain with different anisotropies has been analyzed. Comparing these results with the exact two-spinon spin structure factor of Caux et al. [CKSW12] it can be seen that the qualitative features are again correctly reproduced. For small anisotropy, that is $\Delta \approx J$, the spin structure factor is very similar to the one of the isotropic chain of the previous subsection. In the other limit of $\Delta \approx 0$ the so-called XY Heisenberg chain is obtained. Considering both the reduction of the value of the highest excitations at $q=\pi$ and the 
redistribution of weight when reducing $\Delta$ is correctly reproduced. For small $\Delta$ the weight is located mostly at the higher branch, but it is redistributed to the lower branch for larger anisotropy. In case of the nearly isotropic chain, most weight is found at the lower branch around $q=\pi$. When increasing the cluster size it turns out that finite-size effects are small and do not change the qualitative picture.

However, as for the isotropic Heisenberg chain the spin structure factors obtained with spin CPT show some weight in the "forbidden" area with frequencies $\omega$ smaller than the lower boundary of the spin-wave continua.

\section{\begin{tabular}{l|l}
$J_{1}-J_{2}$ Heisenberg Ladder & B.3
\end{tabular}}

Figure B.3 shows the spin structure factor $S^{z z}$ for the isotropic Heisenberg chain with nearest $\left(J_{1}\right)$ and next-to-nearest $\left(J_{2}\right)$ spin-spin interactions. Thereby the interactions $J_{1}$ and $J_{2}$ compete with each other to induce magnetic order - the system is frustrated. For the application of spin CPT two differences to the two model systems of the previous subsections are important. First of all, when tiling the system into clusters at each end two interaction lines are cut instead of one. The error that is introduced by treating these interactions only within first order perturbation theory is therefore larger. On the other side, frustration should reduce long-range correlations and the self-energy should be more local. A cluster treatment should for this reason work better than before.

Recently, Levarélo and Roux analyzed the $J_{1}-J_{2}$ chain using exact diagonalization and variational calculations in the dimer basis [LR14]. The latter is motivated by the point $J_{2} / J_{1}=0.5$, where the system is described by a fully dimerized ground state. This is the so-called Majumdar-Ghosh point. In their study they derive the two spinon dispersion relations and compare it with exact diagonalization (ED) results for finite chains. Figure B.3 uses the same values $J_{2} / J_{1}$; therefore the spin structure factor of SCPT can be compared to their results.

As in the previous subsections, the spin structure factor qualitatively reproduces the ED results and allows for a smooth spin structure factor if the broadening is increased. In the extreme cases of $J_{2} / J_{1}=0$ and $J_{2} / J_{1}=4$ spin CPT produces weight in the region where the two spinon dispersion of [LR14] does not show any weight. However, close to the Majumdar-Ghosh (MG) point where the system is described by a dimerized ground state, the spin CPT with even cluster sizes shows good agreement with the results of [LR14]. The weight in the "forbidden" region reduces when approaching this point.

Despite the deficiencies of SCPT for spin systems with weak frustration, the $J_{1}-J_{2}$ chain can be treated well with SCPT around the MG point. An use of SCPT as a supplemental tool for ED calculations in frustrated spin systems is promising. The analysis of spin structure factors for two-dimensional frustrated spin systems with spin CPT are left for future studies. 

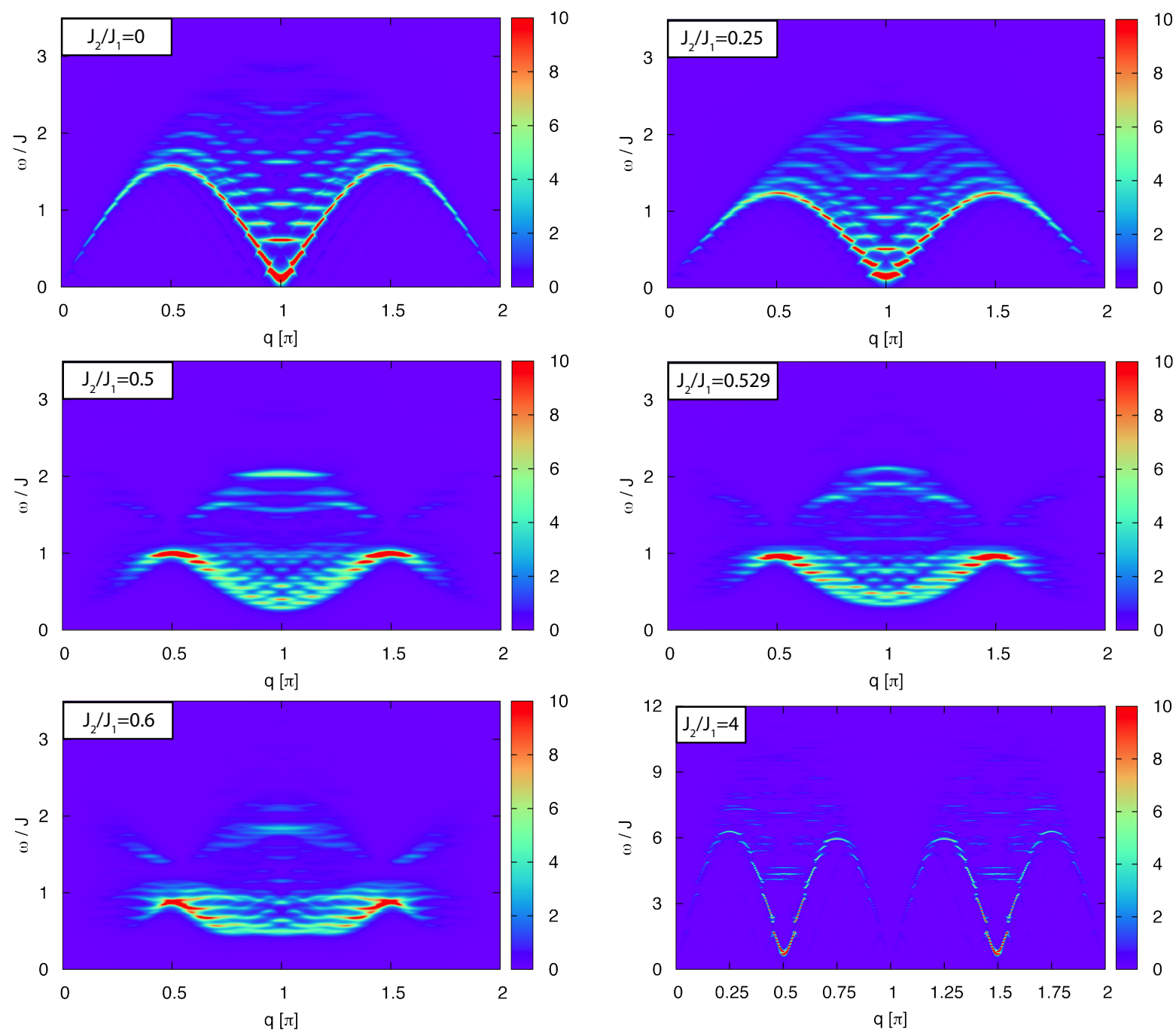

Figure B.3: Spin structure factor $S^{z z}$ of the $J_{1}-J_{2}$ chain for different interaction strengths (from top left to bottom right: $\left.J_{2} / J_{1}=0.0,0.25,0.5,0.529,0.6,4\right)$ using spin CPT with a cluster of length $L=20$. 


\section{Semi-Analytic Evaluation of the Self-Energy Functional}

Here we show the contour deformations that are needed to calculate the sum over Matsubara frequencies of the self-energy functional analytically. The reasoning and calculation closely follows reference [Pot03a].

Starting point for the calculation is the second term of equation (3.2),

$$
I=\operatorname{Tr} \ln \left(\frac{-1}{\mathbf{G}_{\mathbf{0}}^{-1}(\mathbf{t})-\Sigma^{\prime}}\right) .
$$

The non-interacting Green function is given by $\mathbf{G}_{\mathbf{0}}=(\omega+\mu-\mathbf{t})^{-1}$ and the frequency sum hence diverges as $\mathbf{G}(\omega) \propto 1 / \omega$ for large frequencies. In order to take care of this divergence one has to introduce a factor $e^{i \omega 0^{+}}$, as

$$
\int \mathrm{d} \omega \frac{e^{i \omega 0^{+}}}{\omega}=\ln (\omega) e^{i \omega 0^{+}}+\int \mathrm{d} \omega i 0^{+} \frac{e^{i \omega 0^{+}}}{\omega^{2}} .
$$

Therefore

$$
\operatorname{Tr} \ln \left(\frac{-1}{\mathbf{G}_{\mathbf{0}}^{-1}(\mathbf{t})-\Sigma^{\prime}}\right)=T \sum_{n} e^{i \omega_{n} 0^{+}} \operatorname{tr} \ln \left(\frac{-1}{i \omega_{n}+\mu-\mathbf{t}-\Sigma^{\prime}\left(i \omega_{n}\right)}\right),
$$

where the trace Tr has been replaced by a sum over Matsubara frequencies $i \omega_{n}=T \pi(2 n+$ 1 ) and a trace tr over quantum numbers such as spin, cluster and band indices. As the trace is invariant under cyclic permutations one can use $\mathbf{1}=\mathbf{U U}^{\dagger}$ to diagonalize $\mathbf{t}+\boldsymbol{\Sigma}(\omega)$ :

$$
\mathbf{t}+\boldsymbol{\Sigma}(\omega)=\mathbf{U}(\omega) \eta(\omega) \mathbf{U}^{\dagger}(\omega), \quad \eta=\operatorname{diag}\left(\eta_{k}\right) .
$$

Introducing the short-hand notation $g_{k}(\omega)=\left(\omega+\mu-\eta_{k}(\omega)\right)^{-1}$ the expression reads

$$
I=T \sum_{k} \sum_{n} e^{i \omega_{n} 0^{+}} \operatorname{tr} \ln \left(-g_{k}\left(i \omega_{n}\right)\right) .
$$

In the following this sum will be rewritten as a contour integration in complex space. The poles of the Green function $\mathbf{G}(\omega)$ are located on the real axis whereas the Matsubara frequencies lie on the imaginary axis, see figure C.1. Next, one uses the fact that the Matsubara frequencies $\omega_{n}$ are nothing but the poles of the Fermi-Dirac distribution $f^{\mathrm{FD}}(z)=\left(e^{z / T}+1\right)^{-1}$ and the residue theorem, which states that for a suitably chosen function $f(z)$ the contour integration of this function amounts to the sum of their residues: $\oint_{C} f(z) \mathrm{d} z=2 \pi i \sum_{p} \operatorname{Res}\left(f\left(z, z_{p}\right)\right)$. Instead of summing the expression in (C.1) over Matsubara frequencies one can just as well integrate over a function which possesses the suitable residues. Here, this amounts to choosing $f(z)=-1 /(2 \pi i) f^{\mathrm{FD}}(z) \operatorname{tr} \ln \left(-g_{k}(z)\right) e^{z 0^{+}}$ as

$$
\begin{aligned}
\operatorname{Res}\left(f\left(z, \omega_{n}\right)\right) & =\frac{-1}{2 \pi i} \lim _{z \rightarrow \omega_{n}} \frac{z-\omega_{n}}{e^{z / T}+1} \operatorname{tr} \ln \left(-g_{k}(z)\right) e^{z 0^{+}}, \quad e^{\omega_{n} / T}=-1 \\
& =\frac{-T}{2 \pi i} \lim _{z \rightarrow \omega_{n}}\left(\frac{e^{z 0^{+}}}{e^{z / T}} \operatorname{tr} \ln \left(-g_{k}(z)\right)+\frac{z-\omega_{n}}{e^{z / T}+1} \frac{\partial}{\partial z} e^{z 0^{+}} \operatorname{tr} \ln \left(-g_{k}(z)\right)\right) \\
& =\frac{T}{2 \pi i} \operatorname{tr} \ln \left(-g_{k}\left(\omega_{n}\right)\right) e^{\omega_{n} 0^{+}} .
\end{aligned}
$$


From first to second line L'Hopital's rule $\lim _{x \rightarrow x_{0}} f(x) / g(x)=\lim _{x \rightarrow x_{0}} f^{\prime} / g(x)$ has been used as both nominator and denominator go to zero as $z \rightarrow \omega_{n}$. This means that equation (C.1) can be cast in the form

$$
I=\frac{-1}{2 \pi i} \sum_{k} \oint \mathrm{d} \omega e^{\omega 0^{+}} f^{\mathrm{FD}}(\omega) \ln \left(-g_{k}(\omega)\right),
$$

where the contour $C_{1}$ includes the Matsubara frequencies as shown in figure C.1. In the limit $\omega \rightarrow \pm \infty$ the factor $e^{\omega 0^{+}}$and the Fermi function $f^{\mathrm{FD}}(\omega)$ make the integral converge as

$$
\frac{e^{\omega 0^{+}}}{e^{\omega / T}+1} \ln \left(-g_{k}(\omega)\right) \stackrel{\omega \rightarrow \pm \infty}{\longrightarrow} 0
$$
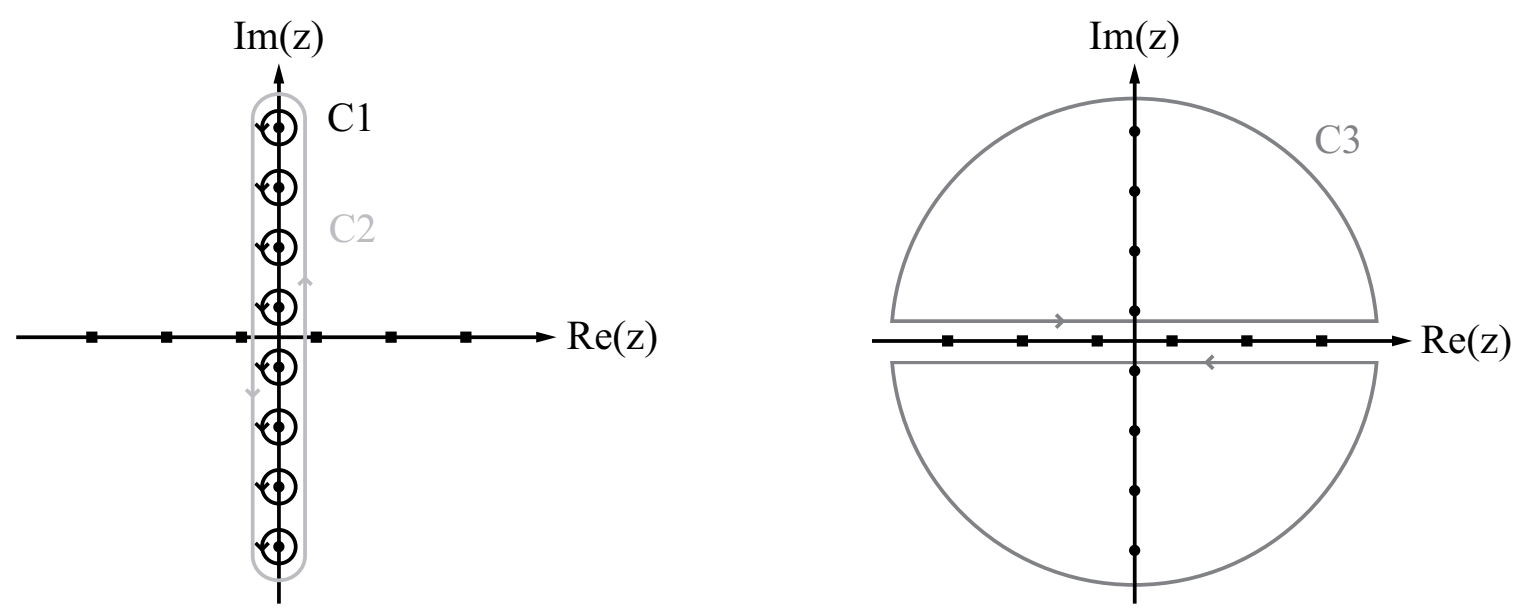

Figure C.1: Contours $C_{1}$ and $C_{2}$ enclose all Matsubara frequencies $\omega_{n}=i \pi T(2 n+1)$ counterclockwise. As the integrand of equation (C.2) goes to zero as $\omega \rightarrow \pm \infty$ the contour can be further deformed into $C_{3}$. Poles of the Green function $G(z)$ are drawn as squares on the real axis.

Using contour $C_{3}$ the integral becomes

$$
\begin{aligned}
I= & -\frac{1}{2 \pi i} \lim _{\delta \rightarrow 0} \sum_{k}\left\{\int_{-\infty}^{\infty} \mathrm{d} \omega e^{(\omega+i \delta) 0^{+}} f^{\mathrm{FD}}(\omega+i \delta) \ln \left(-g_{k}(\omega+i \delta)\right)\right. \\
& \left.+\int_{\infty}^{-\infty} \mathrm{d} \omega e^{(\omega-i \delta) 0^{+}} f^{\mathrm{FD}}(\omega-i \delta) \ln \left(-g_{k}(\omega-i \delta)\right)\right\} \\
= & -\frac{1}{2 \pi i} \sum_{k} \int_{-\infty}^{\infty} \mathrm{d} \omega e^{\omega 0^{+}} f^{\mathrm{FD}}(\omega)\left[\ln \left(-g_{k}\left(\omega+i 0^{+}\right)\right)-\ln \left(-g_{k}\left(\omega-i 0^{+}\right)\right)\right] \\
= & -\frac{1}{\pi} \sum_{k} \int_{-\infty}^{\infty} \mathrm{d} \omega e^{\omega 0^{+}} f^{\mathrm{FD}}(\omega) \operatorname{Im} \ln \left(-g_{k}\left(\omega+i 0^{+}\right)\right) \\
= & -\frac{1}{\pi} \sum_{k} \int_{-\infty}^{\infty} \mathrm{d} \omega f^{\mathrm{FD}}(\omega) \operatorname{Im} \ln \left(-g_{k}\left(\omega+i 0^{+}\right)\right)
\end{aligned}
$$

In the last step the convergence factor $e^{\omega 0^{+}}$has been dropped as the divergence of $g_{k}(\omega)$ encountered previously is no problem anymore.

Using $-1 / \pi \operatorname{Im}\left(g_{k}\left(\omega+i 0^{+}\right)\right) \geq 0$, one can rewrite $g_{k}$ in a polar representation $g_{k}(\omega+$ $\left.i 0^{+}\right)=-r(\omega) e^{i \phi(\omega)}$, with $\phi(\omega) \in(-\pi, \pi]$. This is possible as $g_{k}$ has real poles at $\omega_{m}$ and 
corresponding positive residues $R_{m} g_{k}(\omega)=\sum_{m} R_{m} /\left(\omega-\omega_{m}\right)$. The principal branch of the logarithm then leads to $\operatorname{Im} \ln \left(-g_{k}\left(\omega+i 0^{+}\right)\right)=\phi(\omega)$. As long as $\omega \neq \omega_{m}$, i.e. away from the poles of $g_{k}$, it holds that $\operatorname{Im}\left(-g_{k}\left(\omega+i 0^{+}\right)\right)=0^{+}$, which fixes the phase to $\phi(\omega)=\pi$ if $-g_{k}(\omega)<0$ and to zero if $g_{k}(\omega)>0$. Combined this leads to

$$
\operatorname{Im} \ln \left(-g_{k}\left(\omega+i 0^{+}\right)\right)=\pi \theta\left(g_{k}(\omega)\right)=\pi \theta\left(1 / g_{k}(\omega)\right) .
$$

Right at the poles $\omega_{m}$ the term $\operatorname{Im}\left(-g_{k}\left(\omega+i 0^{+}\right)\right)$diverges, but the phase is still between $-\pi$ and $\pi$. This means that $\phi\left(\omega_{m} \pm 0^{+}\right)=\mp \pi / 2$ and the term remains finite. It can be therefore ignored in the integration over real frequencies $\omega$ as the poles of $g_{k}(\omega)$ are isolated and $I$ reads

$$
-\sum_{k} \int_{-\infty}^{\infty} \mathrm{d} \omega f^{\mathrm{FD}}(\omega) \theta\left(\omega+\mu-\eta_{k}(\omega)\right) .
$$

The Fermi-Dirac distribution can be rewritten as $f^{\mathrm{FD}}(\omega)=\frac{\mathrm{d}}{\mathrm{d} \omega}\left(-T \ln \left(e^{-\omega / T}+1\right)\right)$, hence partial integration leads to

$$
\begin{aligned}
I= & \left.T \sum_{k} \ln \left(e^{-\omega / T}+1\right) \theta\left(\omega+\mu-\eta_{k}(\omega)\right)\right|_{\omega=-\infty} ^{\omega=\infty} \\
& -T \sum_{k} \int_{-\infty}^{\infty} \mathrm{d} \omega \ln \left(e^{-\omega / T}+1\right) \frac{\mathrm{d} \theta\left(\omega+\mu-\eta_{k}(\omega)\right)}{\mathrm{d} \omega} \\
= & -T \sum_{k} \int_{-\infty}^{\infty} \mathrm{d} \omega \ln \left(e^{-\omega / T}+1\right) \frac{\mathrm{d} \theta\left(\omega+\mu-\eta_{k}(\omega)\right)}{\mathrm{d} \omega}
\end{aligned}
$$

To evaluate the derivative of the theta-function one first of all notices that $\theta(\omega+\mu-$ $\left.\eta_{k}(\omega)\right)$ is constant between consecutive poles of $g_{k}(\omega)$. Thus, $\mathrm{d} \theta\left(g_{k}(\omega)\right) / \mathrm{d} \omega$ is non-zero only at zeros and poles of $g_{k}(\omega)$. Rewriting the poles of $g_{k}$ as the zeros of $1 / g_{k}$, this expression can be evaluated as $\delta\left(g_{k}(\omega)\right) g_{k}^{\prime}(\omega)=\sum_{z} g_{k}^{\prime}\left(\omega_{z}\right) /\left|g_{k}^{\prime}\left(\omega_{z}\right)\right| \delta\left(\omega-\omega_{z}\right)$ for the zeros and $\delta\left(1 / g_{k}(\omega)\right)\left(1 / g_{k}\right)^{\prime}(\omega)=\sum_{p}\left(1 / g_{k}\right)^{\prime}\left(\omega_{p}\right) /\left|\left(1 / g_{k}\right)^{\prime}(\omega)\right| \delta\left(\omega-\omega_{p}\right)$ for the poles. Here, the prime denotes the derivative with respect to $\omega$ and is not used to indicate cluster quantities. Consequently it amounts to summing over zeros $\omega_{l}$ and poles $\omega_{p}$

$$
\frac{\mathrm{d} \theta\left(\omega+\mu-\eta_{k}(\omega)\right)}{\mathrm{d} \omega}=\sum_{z} \delta\left(\omega-\omega_{z}\right)-\sum_{p} \delta\left(\omega-\omega_{p}\right),
$$

where it was used that zeros of $g_{k}$ are poles of $\mathbf{G}$ and hence have positive residues, whereas poles of $g_{k}$ (and thus zeros of $G$ ) can be identified with the poles of the self-energy $\boldsymbol{\Sigma}$ and have negative residues.

The diagonal elements of $\mathbf{G}$ have the same poles respectively zeros and accordingly the sum over $k$ can be replaced by a multiplication with the dimension of $\mathbf{G}$, which in the case of a $L$-site reference cluster with spin up and spin down electrons amounts to $2 L$ :

$$
\begin{aligned}
I & =-\sum_{k, z} T \ln \left(1+e^{\omega_{z} / T}\right)+\sum_{k, p} T \ln \left(1+e^{-\omega_{p} / T}\right) \\
& =-2 L\{\sum_{z} T \ln \left(1+e^{\omega_{z} / T}\right)+\underbrace{\sum_{p} T \ln \left(1+e^{-\omega_{p} / T}\right)}_{P_{\Sigma}}\} .
\end{aligned}
$$


The second term sums all poles of the self-energy and is abbreviated by $P_{\Sigma}$. It should be noted that the self-energy $\Sigma$ was chosen to be $t^{\prime}$-representable (a cluster self-energy). In the first term the sum goes over zeros of $g_{k}$ which are the poles of $\mathbf{G}(\omega)=\left(\mathbf{G}_{\mathbf{0}}{ }^{-1}-\mathbf{\Sigma}(\omega)\right)^{-1}$. This reminds of the grand potential of a system which consists of quasi-particles with energies given by exactly these poles but with unity weight.

Turning back to the self-energy functional as it is given in equation (3.2), the third term still has to be transformed. As its structure is the same as the one of the second term, the calculation proceeds identically and leads to

$$
I_{3}=-2 L \sum_{z} T \ln \left(1+e^{\omega_{z}^{\prime} / T}\right)-2 L P_{\Sigma}
$$

Compared to (C.3) the first term includes a sum over $\omega_{z}^{\prime}$, which in this case are the poles of the cluster Green function $\mathbf{G}^{\prime}(\omega) . \quad P_{\Sigma}$ is identical to the term derived for $I$ as the self-energy of the reference system is also used in the original system.

Putting all three terms together the self-energy functional reads

$$
\begin{aligned}
\Omega\left[\Sigma\left(\mathbf{t}^{\prime}\right)\right]= & -T \ln \sum_{m} e^{-\left(E_{m}^{\prime}-\mu N_{m}^{\prime}\right) / T}-2 L \sum_{m} T \ln \left(1+e^{-\omega_{m} / T}\right) \\
& +2 L \sum_{m} T \ln \left(1+e^{-\omega_{m}^{\prime} / T}\right) .
\end{aligned}
$$

When evaluating the self-energy functional at finite temperatures, one usually has to express the energies with respect to the lowest cluster energy $E_{0}^{\prime}$ in order to be able to evaluate the exponentials. This resolves the problem of negative $E_{m}^{\prime}-\mu N_{m}^{\prime}$ that would lead to huge exponents. For example, the first term can be transformed as

$$
-T \sum_{m} e^{-\left(E_{m}^{\prime}-\mu N_{m}^{\prime}\right) / T}=E_{0}^{\prime}-T \sum_{m} e^{-\left(E_{m}^{\prime}-\mu N_{m}^{\prime}-E_{0}^{\prime}\right) / T}
$$

and

$$
\ln \left(1+e^{-\omega_{m}^{\prime} / T}\right)+\omega_{0}^{\prime} / T=\ln \left(e^{\omega_{0}^{\prime} / T}+e^{-\left(\omega_{m}^{\prime}-\omega_{0}^{\prime}\right) / T}\right) .
$$

As $\omega_{0}^{\prime}<0$ and $e^{\omega_{0}^{\prime} / T} \ll 1$, if the temperature is sufficiently small, the self-energy functional can be calculated numerically as

$$
\begin{aligned}
\Omega\left[\Sigma\left(\mathbf{t}^{\prime}\right)\right]= & E_{0}^{\prime}-T \sum_{m} e^{-\left(E_{m}^{\prime}-\mu N_{m}^{\prime}-E_{0}^{\prime}\right) / T}-2 L \sum_{m} T \ln \left(e^{\omega_{0}^{\prime} / T}+e^{-\left(\omega_{m}^{\prime}-\omega_{0}^{\prime}\right) / T}\right) \\
& +2 L \sum_{m} \ln \left(e^{\omega_{0}^{\prime} / T}+e^{-\left(\omega_{m}-\omega_{0}^{\prime}\right) / T}\right) .
\end{aligned}
$$

At zero temperature this equation simplifies to

$$
\Omega=E_{0}^{\prime}-2 L \sum_{m, \omega_{m}<0} \omega_{m}^{\prime}+2 L \sum_{m, \omega_{m}<0} \omega_{m} .
$$




\section{Tight Binding Theory for Néel and Collinear Order}

In order to understand the nature of the electron- and hole-pockets of the Fermi surface at small inter-chain hopping $t_{\perp}$ we will in the following consider simple tight binding theory to investigate the effect of Néel and Stripe order. Although the Fermi surface pockets are in our case supposed to be a result of many-body interactions, assuming one of the possibly emerging orders as a starting point for tight binding calculations might allow for deductions with respect to the scattering processes involved. Furthermore, at least for doped two-dimensional Hubbard models, which are commonly used to model cuprate superconductors, mean-field calculations are capable of showing closed regions of the Fermi surface and reproducing electron- and hole-pockets [Ovc08].

Considering only the hopping terms of the model, one performs a Fourier transformation and obtains the tight-binding dispersion

$$
\epsilon(\mathbf{k})=-2\left(t \cos \left(\mathbf{k}_{x}\right)+t_{\perp} \cos \left(\mathbf{k}_{y}\right)\right)+t_{\perp} \cos \left(\mathbf{k}_{x}\right) \cos \left(\mathbf{k}_{y}\right)
$$

As the formation of electron- and hole-pockets is an effect of Coulomb interaction $U$, which causes different types of scattering processes, we first of all consider two different possible orders of the lattice: Néel-like order with a ordering vector of $(\pi, \pi)$ and stripe order with a vector $(\pi, 0)$. For both orders the lattice is devided into two sublattices $\mathrm{A}$ and B as shown in figure D.1.
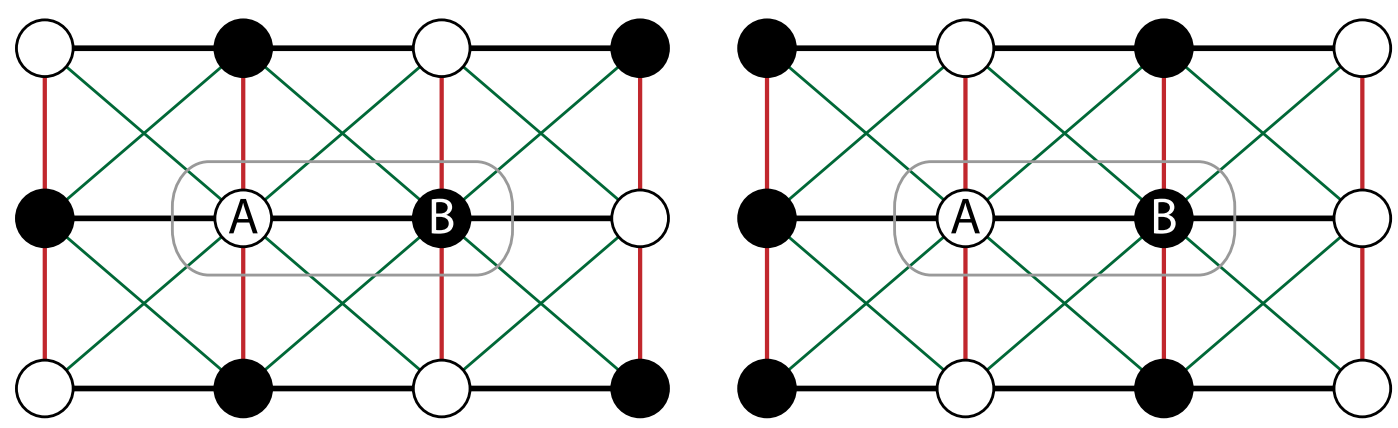

Figure D.1: Deviding the lattice into sublattices A and B for Néel-like (left) and stripe (right) order.

The unit cell now contains two atoms, one of each sublattice and defines the tight binding Hamiltonians $\mathcal{H}_{\mathrm{AF}}$ and $\mathcal{H}_{\text {stripe }}$. To distinguish both sublattices, we call electron creation and annihilation operators on sublattice $\mathrm{A}$ by $a_{i}^{(\dagger)}$ and on B by $b_{i}^{(\dagger)}$. 


$$
\begin{aligned}
\mathcal{H}_{\mathrm{AF}}= & \sum_{i}\left[t\left(a_{i}^{\dagger} b_{i}+b_{i}^{\dagger} a_{i}+a_{i}^{\dagger} b_{i-2 e_{x}}+b_{i}^{\dagger} a_{i+2 e_{x}}\right)\right. \\
& +t_{\perp}\left(a_{i}^{\dagger} b_{i-e_{x}+e_{y}}+b_{i}^{\dagger} a_{i+e_{x}+e_{y}}+a_{i}^{\dagger} b_{i-e_{x}-e_{y}}+b_{i}^{\dagger} a_{i+e_{x}-e_{y}}\right) \\
& \left.+\sum_{c=a, b} t^{\prime}\left(c_{i}^{\dagger} c_{i-e_{x}+e_{y}}+c_{i}^{\dagger} c_{i-e_{x}-e_{y}}+c_{i}^{\dagger} c_{i+e_{x}+e_{y}}+c_{i}^{\dagger} c_{i+e_{x}-e_{y}}\right)\right] .
\end{aligned}
$$

One continues by performing a Fourier transformation to momentum space via

$$
c_{j}^{(\dagger)}=\frac{1}{\sqrt{V}} \sum_{\mathbf{k}} e^{(-) i \mathbf{r}_{j} \mathbf{k}} c_{k}^{(\dagger)}
$$

and using that $\frac{1}{V} \sum_{j} e^{i \mathbf{r}_{j}(\mathbf{k}-\mathbf{q})}=\delta(\mathbf{k}-\mathbf{q})$.

$$
\begin{aligned}
\mathcal{H}_{\mathrm{AF}}= & \sum_{\mathbf{k}}\left[t\left(a_{\mathbf{k}}^{\dagger} b_{\mathbf{k}}\left[1+e^{-2 i \mathbf{k}_{x}}\right]+b_{\mathbf{k}}^{\dagger} a_{\mathbf{k}}\left[1+e^{2 i \mathbf{k}_{x}}\right]\right)\right. \\
& +t_{\perp}\left(a_{\mathbf{k}}^{\dagger} b_{\mathbf{k}}\left[e^{-i \mathbf{k}_{x}+i \mathbf{k}_{y}}+e^{-i \mathbf{k}_{x}-i \mathbf{k}_{y}}\right]+b_{\mathbf{k}}^{\dagger} a_{\mathbf{k}}\left[e^{i \mathbf{k}_{x}+i \mathbf{k}_{y}}+e^{i \mathbf{k}_{x}-i \mathbf{k}_{y}}\right]\right) \\
& \left.+t^{\prime} \sum_{c=a, b} c_{\mathbf{k}}^{\dagger} c_{\mathbf{k}}\left(e^{-i \mathbf{k}_{x}+i \mathbf{k}_{y}}+e^{-i \mathbf{k}_{x}-i \mathbf{k}_{y}}+e^{i \mathbf{k}_{x}+i \mathbf{k}_{y}}+e^{i \mathbf{k}_{x}-i \mathbf{k}_{y}}\right)\right] \\
= & \left(\begin{array}{cc}
4 \cos \left(\mathbf{k}_{x}\right) \cos \left(\mathbf{k}_{y}\right) t^{\prime} & 2 e^{-i \mathbf{k}_{x}}\left(\cos \left(\mathbf{k}_{x}\right) t+\cos \left(\mathbf{k}_{y}\right) t_{\perp}\right) \\
2 e^{i \mathbf{k}_{x}}\left(\cos \left(\mathbf{k}_{x}\right) t+\cos \left(\mathbf{k}_{y}\right) t_{\perp}\right) & 4 \cos \left(\mathbf{k}_{x}\right) \cos \left(\mathbf{k}_{y}\right) t^{\prime}
\end{array}\right) .
\end{aligned}
$$

Diagonalization of this tight binding Hamiltonian leads to the dispersion relation

$$
E_{ \pm}(\mathbf{k})=4 \cos \left(\mathbf{k}_{x}\right) \cos \left(\mathbf{k}_{y}\right) t^{\prime} \pm 2 \sqrt{\left(\cos \left(\mathbf{k}_{x}\right) t+\cos \left(\mathbf{k}_{y}\right) t_{\perp}\right)^{2}+4\left(\cos \left(\mathbf{k}_{x}\right) \cos \left(\mathbf{k}_{y}\right) t^{\prime}\right)^{2}} .
$$

For the model used to model the crossover between one and two dimensions, the diagonal hopping is proportional to the hopping perpendicular to the one-dimensional chains. Especially, for the lattice with small frustration $t^{\prime}=-t_{\perp} / 4$ this reduces the dispersion to

$$
\epsilon_{ \pm}(\mathbf{k})=-\cos \left(\mathbf{k}_{x}\right) \cos \left(\mathbf{k}_{y}\right) t_{\perp} \pm \sqrt{4\left(\cos \left(\mathbf{k}_{x}\right) t+\cos \left(\mathbf{k}_{y}\right) t_{\perp}\right)^{2}+\left(\cos \left(\mathbf{k}_{x}\right) \cos \left(\mathbf{k}_{y}\right) t_{\perp}\right)^{2}}
$$

At zero energy the condition $\cos \left(\mathbf{k}_{x}\right) t=-\cos \left(\mathbf{k}_{y}\right) t_{\perp}$ has to be fulfilled, which simply produces the non-interacting dispersion of the model, which was already shown in section 2.1. In figure D.2, contour plots of $\epsilon_{ \pm}(\mathbf{k})$ away from zero energy show closed structures that are located along the non-interacting dispersion of the original model. However, these "pockets" have a different symmetry than those of the interacting Fermi surface close to the metal insulator transition. The Fermi surface pockets showed a symmetry with respect to $\mathbf{k}_{x}= \pm \pi / 2$, which is a main difference to the structures of the non-interacting dispersion. As Néel order is obtained as a result of antiferromagnetic scattering processes, it is not surprising that the splitting of the two branches of the dispersion happens at points which are connected by the antiferromagnetic ordering vector $(\pi, \pi)$. Due to the frustration, which is added to the system by choosing $t, \neq 0$, one is left with only four points at $( \pm \pi / 2, \pm \pi / 2)$ and $( \pm \pi / 2, \mp \pi / 2)$, which fulfill this condition. The fact that the 

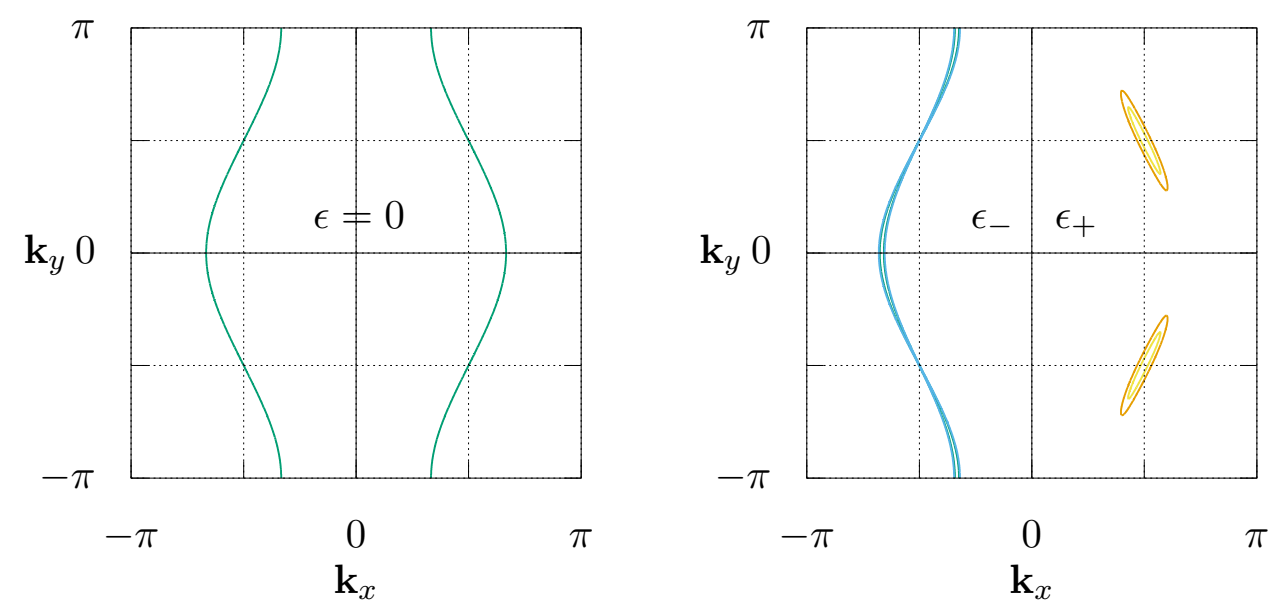

Figure D.2: Contour plots of the tight binding dispersion of the Néel ordered lattice. The line of zero energy is shown on the left, on the right equi-energy lines at $-0.005,-0.01,0.1$ and 0.2 show closed regions, which are reminiscent of the Fermi pockets of section 4.1.

symmetry of the Fermi surface pockets is not reproduced by assuming Néel order leads to the proposal of collinear ordering, which might be caused by "one-dimensional" (e.g. Umklapp) scattering with a $(\pi, 0)$ order vector.

In this case the lattice is again split into two sublattices according to the right panel of figure D.1 and one can use again different operators for each of the two sublattices. The Hamiltonian reads now:

$$
\begin{aligned}
\mathcal{H}_{\text {stripe }}= & \sum_{i}\left[t\left(a_{i}^{\dagger} b_{i}+b_{i}^{\dagger} a_{i}+a_{i}^{\dagger} b_{i+2 e_{x}}+b_{i}^{\dagger} a_{i-2 e_{x}}\right)\right. \\
& +t_{\perp}\left(a_{i}^{\dagger} a_{i+e_{y}}+a_{i}^{\dagger} a_{i-e_{y}}+b_{i}^{\dagger} b_{i+e_{y}}+b_{i}^{\dagger} b_{i-e_{y}}\right) \\
& +t^{\prime}\left(a_{i}^{\dagger} b_{i+2 e_{x}+e_{y}}+a_{i}^{\dagger} b_{i+2 e_{x}-e_{y}}+a_{i}^{\dagger} b_{i+e_{y}}+a_{i}^{\dagger} b_{i-e_{y}}\right. \\
& \left.\left.+b_{i}^{\dagger} a_{i-2 e_{x}+e_{y}}+b_{i}^{\dagger} a_{i-2 e_{x}-e_{y}}+b_{i}^{\dagger} a_{i+e_{y}}+b_{i}^{\dagger} a_{i-e_{y}}\right)\right] .
\end{aligned}
$$

Analogous to the previous calculation one Fourier transforms into momentum space and obtains

$$
\begin{aligned}
\mathcal{H}_{\text {stripe }}= & \sum_{\mathbf{k}}\left[t\left(a_{\mathbf{k}}^{\dagger} b_{\mathbf{k}}\left[1+e^{2 i \mathbf{k}_{x}}\right]+b_{\mathbf{k}}^{\dagger} a_{\mathbf{k}}\left[1+e^{-2 i \mathbf{k}_{x}}\right]\right)\right. \\
& +t_{\perp}\left(a_{\mathbf{k}}^{\dagger} a_{\mathbf{k}}\left[e^{i \mathbf{k}_{y}}+e^{-i \mathbf{k}_{y}}\right]+b_{\mathbf{k}}^{\dagger} b_{\mathbf{k}}\left[e^{i \mathbf{k}_{y}}+e^{-i \mathbf{k}_{y}}\right]\right) \\
& +t^{\prime}\left(a_{\mathbf{k}}^{\dagger} b_{\mathbf{k}}\left\{e^{i \mathbf{k}_{y}}+e^{-i \mathbf{k}_{y}}+e^{2 i \mathbf{k}_{x}+i \mathbf{k}_{y}}+e^{2 i \mathbf{k}_{x}-i \mathbf{k}_{y}}\right\}\right. \\
& \left.\left.+b_{\mathbf{k}}^{\dagger} a_{\mathbf{k}}\left\{e^{i \mathbf{k}_{y}}+e^{-i \mathbf{k}_{y}}+e^{-2 i \mathbf{k}_{x}+i \mathbf{k}_{y}}+e^{-2 i \mathbf{k}_{x}-i \mathbf{k}_{y}}\right\}\right)\right] \\
= & \left(\begin{array}{cc}
2 \cos \left(\mathbf{k}_{y}\right) t_{\perp} & 2 e^{i \mathbf{k}_{x}}+4 e^{i \mathbf{k}_{x}} \cos \left(\mathbf{k}_{x}\right) \cos \left(\mathbf{k}_{y}\right) t^{\prime}
\end{array}\right) .
\end{aligned}
$$


Finally, the two branches of the dispersion read:

$$
E_{ \pm}(\mathbf{k})=2 \cos \left(\mathbf{k}_{y}\right) t_{\perp} \pm 2 \sqrt{\left(\cos ^{2}\left(\mathbf{k}_{x}\right)\left(t+2 \cos \left(\mathbf{k}_{y}\right) t^{\prime}\right)^{2}+\cos ^{2}\left(\mathbf{k}_{y}\right) t_{\perp}\right)^{2}}
$$

For the lattice with frustration $t^{\prime}=-t_{\perp} / 4$ this reduces to

$$
\epsilon_{ \pm}(\mathbf{k})=2 \cos \left(\mathbf{k}_{y}\right) t_{\perp} \pm 2 \sqrt{\cos ^{2}\left(\mathbf{k}_{x}\right)\left(t-\frac{t_{\perp}}{2} \cos \left(\mathbf{k}_{y}\right)\right)^{2}+\cos ^{2}\left(\mathbf{k}_{y}\right) t_{\perp}^{2}}
$$
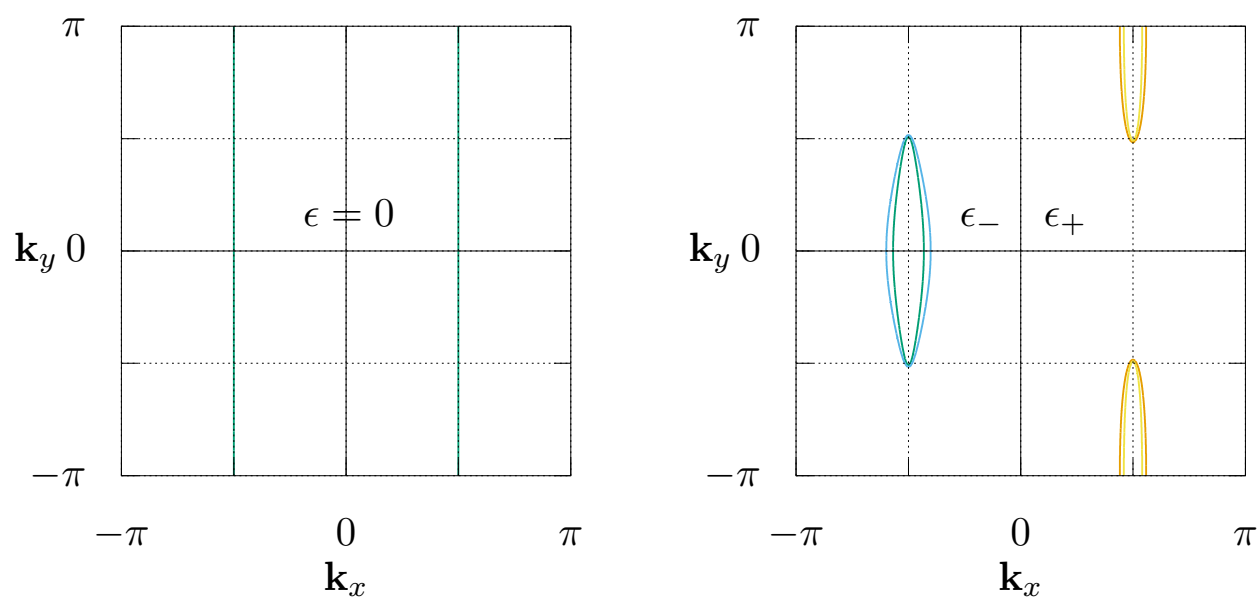

Figure D.3: Contour plots of the tight binding dispersion of the stripe ordered lattice. The line of zero energy is shown on the left, on the right equi-energy lines at $-0.05,-0.1,0.05$ and 0.1 show closed regions, which are symmetric around $\pm \pi / 2$.

In contrast to the Néel order, this time one obtains the one-dimensional dispersion at zero energy. As shown in the contour plot in figure D.3, both branches of the dispersion form structures away from zero energy, that have a mirror symmetry around $\mathbf{k}_{x}= \pm \pi / 2$ and their geometry resembles the Fermi surface pockets close to the metal-insulator transition for small inter-chain hoppings. Another difference to the "AF" dispersion consists in the fact that both branches of the dispersion touch at zero energy, but $\epsilon_{+}(\mathbf{k}) \geq 0$ and $\epsilon_{-}(\mathbf{k}) \leq 0$. 


\section{The Mott Transition within Dynamical Impurity Approximation}

One of the easiest quantum cluster techniques that allow for the coexistence of metal and insulator phases and therefore for a first order metal-insulator transition at finite temperatures for the Hubbard model is the so-called dynamical impurity approximation (DIA)[Pot03a]. It was introduced by Potthoff in 2003 and is also based on self-energy functional theory. Actually, it can be viewed as variational cluster approximation with a specific choice of the cluster: As the necessary reference system only has to have the same interaction part as the original model, not only a tiling of the lattice into clusters, but also coupling of the correlated sites to additional (non-interacting) bath sites is allowed. The DIA now consists in choosing the minimal cluster that also contains bath sites, namely one correlated site coupled to one bath site, and building up the lattice by periodically repeating this cluster, see figure E.1. It can be shown [Pot11], that coupling to infinitely many bath sites instead of only one the variational principle amounts to the self-consistency condition of dynamical mean-field theory (DMFT). Although DMFT can be formulated with a finite number of bath sites [CK94], too, it is not clear, that the two techniques become the same. Nevertheless, the results obtained by Potthoff [Pot03a] for the unfrustrated, isotropic Hubbard model are qualitatively comparable to those of DMFT.
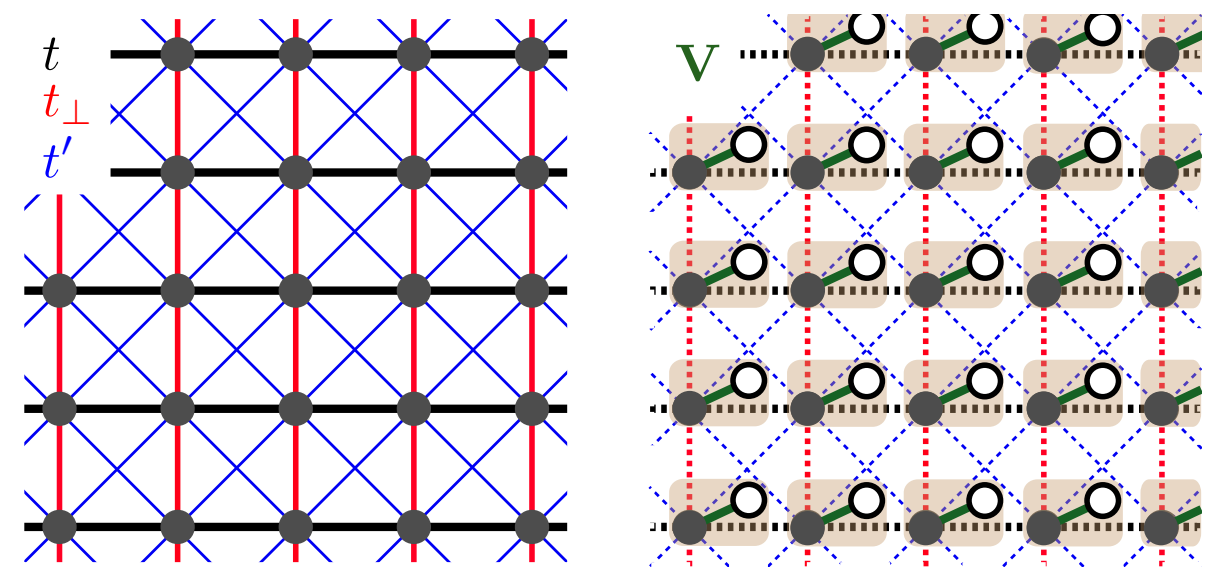

Figure E.1: Sketch of the lattice model (left panel) and the dynamical impurity approximation. The reference clusters consist of a interacting cluster site and a non-interacting bath site that are coupled with a hopping term of strength $V$.

Compared to a single-site cluster, which amounts to approximating the system with the self-energy of the atomic problem, electrons on the correlated site can stay for a finite time on the bath site and can hence avoid the payoff of $U$ due to double occupancy on the correlated site. Due to the hybridization strength $V$ between correlated and bath sites, which is treated as an additional variational parameter in the saddle point search, it is now possible to achieve first order transitions. 
Strong fluctuations between correlated and bath site can lead to a certain double occupancy on the correlated site. In contrast, at weak hybridization the electrons try to avoid double occupancy due to the Coulomb repulsion on the correlated site. Around the critical interaction strength $U_{c}$ it is possible to realize both solutions with different values of $V$.

The DIA is now used for the frustrated anisotropic Hubbard model to revisit the Mott transition. Figure E.2 shows the self-energy functional as a function of hybridization strength $V$. The chemical potential of the cluster $\mu^{\prime}$ is treated as a variational parameter and the chemical potential $\mu$ is chosen such that half-filling is kept for each value of $V$. For large values of $U / t$, there exists only one stationary point for $V=0$ (a minimum with respect to $V$ ), but once one crosses the critical value $U_{c}$ an additional solution at finite $V$ exists. Both solutions exist down to $U=0$.
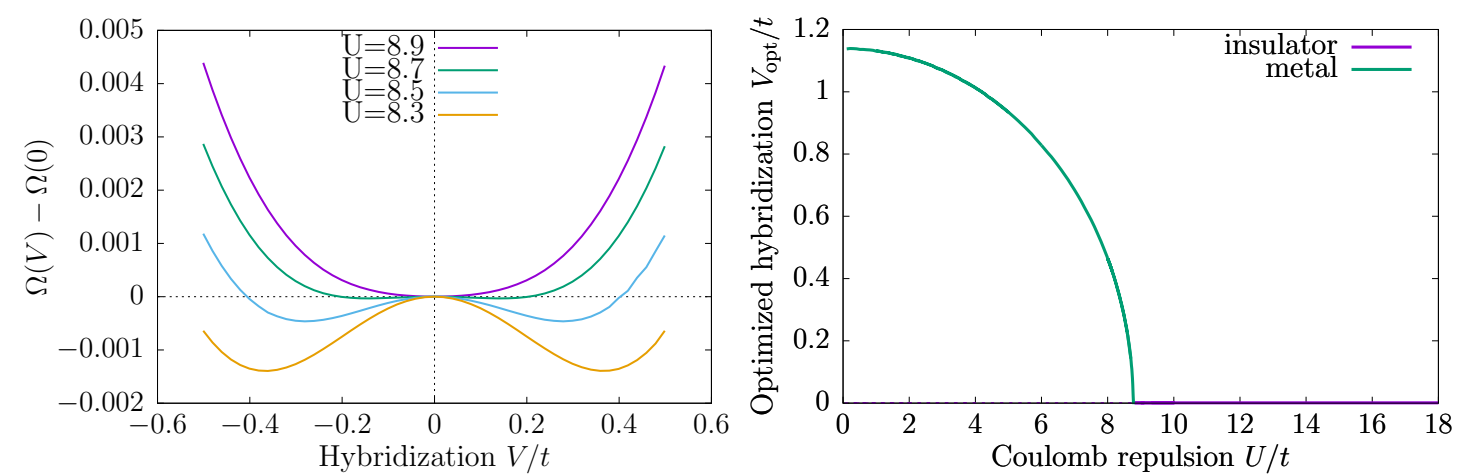

Figure E.2: Left: Grand potential $\Omega$ per site as function of hybridization strength $V$ for different values of $U$ around $U_{c}$ at $t_{\perp}=0.2$. Right: Hybridization strength $V$ of the stationary points as a function of $U$.

The $n$-vs. $-\mu$ curve shows a plateau for the solution with $V=0$ which amounts to a vanishing compressibility - the system is insulating. For the other solution $V$ is finite and it can be identified to be metallic since the electron density has a non-zero gradient as a function of $\mu$.

This can be further confirmed by calculating the (local) density of states (DOS) of the correlated site via

$$
\operatorname{DOS}(\omega)=\frac{1}{N_{k}} \sum_{k} A(k, \omega)
$$

for both solutions. The DOS of the insulating solution shows a gap both for $U<U_{c}$ and for $U \lesssim U_{c}$. Instead, for the metallic solution a finite density of states is found in the middle of the two Hubbard bands around $E=0$ for any $U<U_{c}$. For smaller $U$ weight is reshuffled from the outer bands into the region around $E_{0}$. This picture of realizing the Mott transition resembles the pre-formed gap scenario of dynamical mean-field theory. There, the two Hubbard bands are already well separated close to the transition and the weight of the quasiparticle peak at $\omega=0$ vanishes at $U_{c}$.

To know which solution is realized for $U<U_{c}$, the ground-state energy density $E_{0}$ of both solutions is calculated and compared. It turns out, that for all values of $U$ the stable solution is the one with a minimum with respect to $V$, which means that there is a transition from metal to insulator at $U_{c}$. Choosing the solution according to the hierarchy of stationary points introduced by Potthoff in reference [Pot06b] also amounts to taking 
the metallic solution for $U<U_{c}$ as the other stationary point is a trivial one and has to be disregarded. As expected, in the limit $U \rightarrow 0$ the energies of both solutions go to the same non-interacting value, which amounts to the free electron gas.

Finally, the double occupancy $d$, which is often used like an order parameter to discriminate metal from insulator [RCK99, ON03], can be obtained as $d=\frac{\partial \Omega}{\partial U}$, see figure E.3. At the transition point, $d$ of the metallic solution shows to be proportional to $U$ and clearly different from $d_{\text {INS }}$, but in contrast to the expectation for a "typical" order parameter it is not zero in the insulating phase. This behavior does not change qualitatively for different values of inter-chain coupling $t_{\perp}$.
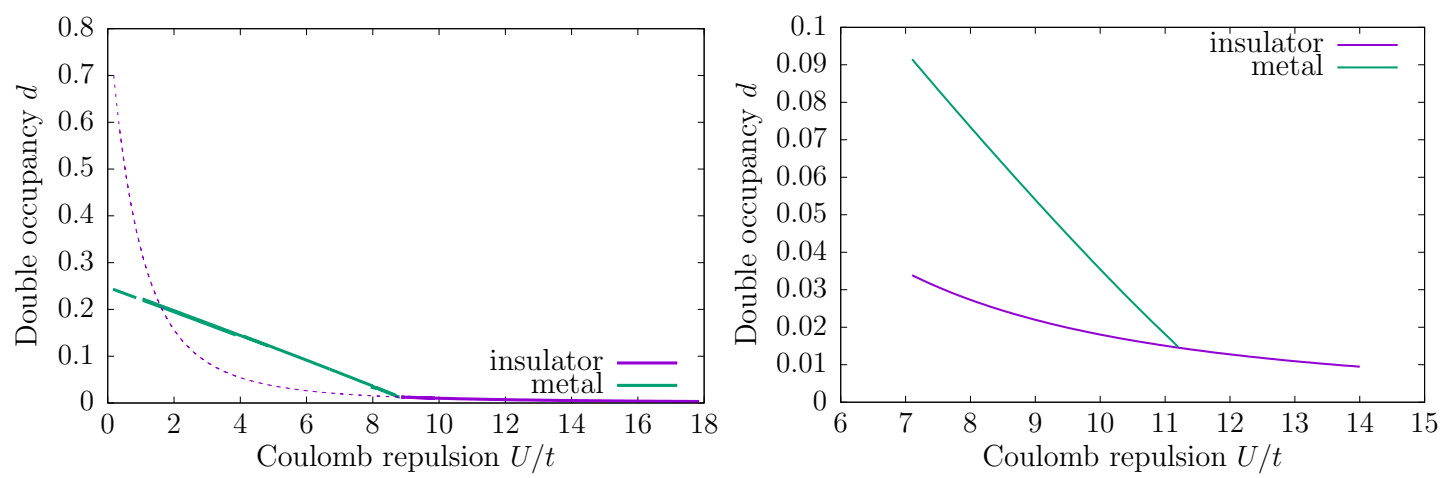

Figure E.3: Double occupancy $d$ per site as function of $U$ for both solutions: metal $(V \neq 0)$ and insulator $(V=0)$. However, the metallic solution is the one with lower $E_{0}$ for $U<U_{c}$.

If one investigates the spectral function $A(\mathbf{k}, \omega)$ of the metallic solution, the Fermi surface (FS) is given by the energy cut at $\omega=0$. In reference [RA12] the formation of electronand hole-pockets in the metallic phase was attributed to emerge due to Umklapp scattering at small $t_{\perp}$. The cluster used in DIA does not include any spatial fluctuations inside the cluster that would allow such scattering processes. As a consequence no electron- and hole-pockets are present in the Fermi surface. Only the use of clusters that have finite spatial extend and which include a coupling to bath sites would enable a study of both the order of the Mott transition and the Fermi surface topology inside the metallic phase. This is done in sections 4.2 and 4.3 .

When comparing to finite-temperature DIA studies on the Hubbard model [Pot03a, Poz04, HEP16] a qualitative difference is visible at zero temperature. At finite temperatures, two minima in the self-energy functional can be identified in a region $U_{c_{1}}<U<U_{c_{2}}$ which is then identified as a coexistence region of metal and insulator. This results in a phase diagram comparable to the one known from dynamical mean-field theory.

However, the scenario presented here at zero temperature is different, as only one stationary point with $V \neq 0$ is obtained at $U<U_{c}$ corresponding to the metallic solution. For $U>U_{c}$ the only stationary point exists at $V=0$ and not at a non-zero value like at finite temperatures. This solution corresponds to the insulator.

To illustrate this difference, figure E.4 shows the self-energy functional for the Hubbard model at $t_{\perp} / t=0.2$ and an interaction strength $U<U_{c}$ in the coexistence region at temperatures $0 \leq T<T_{c}$. At finite temperature, three non-trivial stationary points are found, which correspond to the insulating solution $\left(V_{\min , 1}\right)$, the metallic solution $\left(V_{\min , 2}\right)$ and an unstable solution $\left(V_{\max }\right)$. Extrapolating the position of the critical interaction 
strengths $U_{c_{1}}, U_{c}, U_{c_{2}}$ of the finite-temperature results to zero temperature, a finite coexistence region between $U_{c_{1}}$ and $U_{c}=U_{c_{2}}$ is expected [Poz04, HEP16]. In contrast to this expectation, at zero temperature only the trivial stationary point at $V=0$ corresponding to an insulating solution for all interaction strengths and one minimum at a finite hybridization strength exist for $U<U_{c}$.

When investigating the self-energy functional for very small temperatures, the position of the first minimum at $V_{\min , 1}$ as well as the relative values $\Omega(V=0)-\Omega\left(V_{\min , 1}\right)$ and $\Omega\left(V_{\max }\right)-\Omega\left(V_{\min , 1}\right)$ can be plotted as a function of temperature, see right panel of figure E.4. The extrapolation of the relative depth of the minimum of the SEF suggests that the solution vanishes at zero temperature. Therefore only the metallic solution at $V_{\min , 2}$ and the (trivial) insulating solution at $V=0$ is left. As a consequence, no coexistence region with two solutions with non-zero hybridization strengths can be found.

This qualitative difference of the structure of the self-energy functional at zero and at finite temperature as well as its implications on the order of the Mott transition at zero temperature needs further investigation, which is left for future studies.
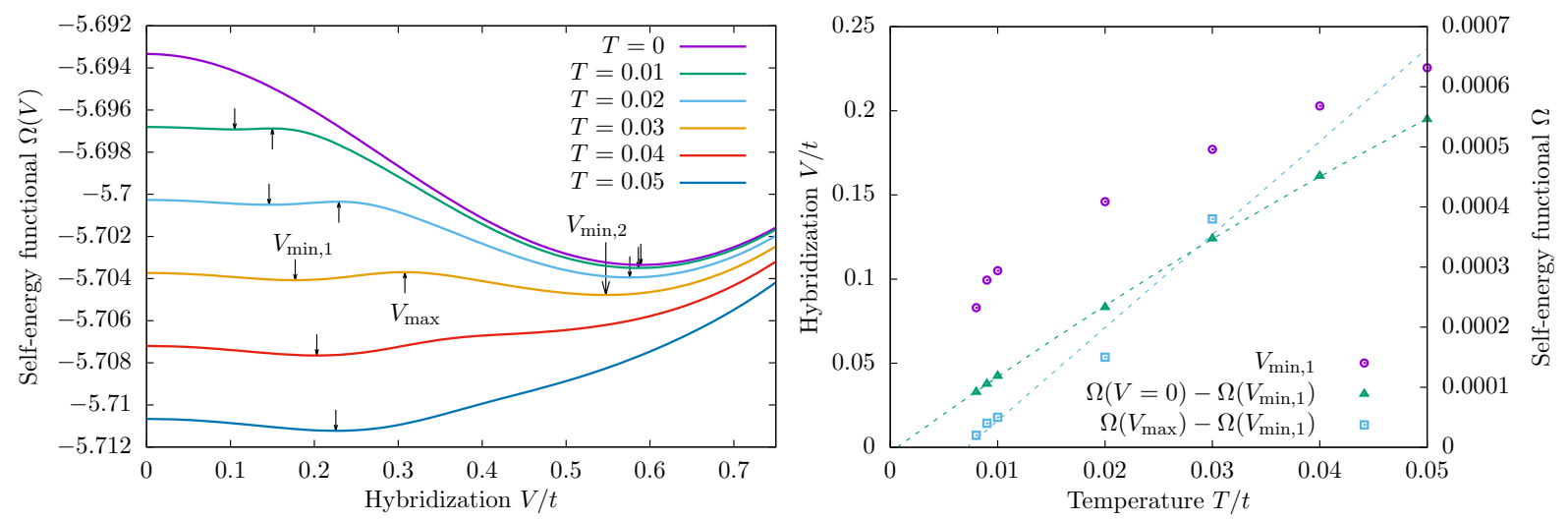

Figure E.4: Left panel: Self-energy functional as a function of hybridization strength for the Hubbard model with $t_{\perp} / t=0.2, U / t=7.5<U_{c} / t$. The different non-trivial stationary points are indicated with arrows. At zero temperature only one stable solution is left corresponding to the metal. For $T=0.01$ an insulating solution with small $V \neq 0$ exists in addition and for $T>T_{c}$ only the insulating solution remains. Right panel: Position of the minimum $V_{\min , 1}$ and relative value of the self-energy functional $\Omega(V=0)-\Omega\left(V_{\min , 1}\right)$ and $\Omega\left(V_{\max }\right)-\Omega\left(V_{\min , 1}\right)$ as a function of temperature. Extrapolation to zero temperature suggests an absence of the insulating solution for $T=0$. 


\section{Preliminary Studies of Local s-Wave Pairing in the Kondo Lattice Model}

In this appendix some easy calculations at the single-site level are shown in order to better understand the effect of adding a superconducting Weiss field. Then, a $2 \times 2$ cluster is investigated with exact diagonalization to show the changes due to the inclusion of spatial fluctuations.

\section{An Isolated Kondo Site $\mid F .1$}

Looking at an isolated Kondo site, i.e. a single site coupled to an f-electron spin, the Hilbert space has a size of 8 . It contains the states

$$
\{|\Uparrow\rangle,|\Uparrow \downarrow\rangle,|\Uparrow \uparrow\rangle,|\Uparrow \uparrow \downarrow\rangle,|\Downarrow\rangle,|\Downarrow \downarrow\rangle,|\Downarrow \uparrow\rangle,|\Downarrow \uparrow \downarrow\rangle\},
$$

where the bold arrows denote the orientation of the f-spin and the thin arrows stand for spin-up or spin-down electrons. As the kinetic term is gone, we are left with the superconducting pair annihilation and creation operations and the Kondo exchange $J$ between electrons and localized spins:

$$
\begin{aligned}
\mathcal{H}_{\mathrm{SK}} & =J \mathbf{S} \cdot \sigma+\Delta\left(c_{\uparrow} c_{\downarrow}+c_{\uparrow}^{\dagger} c_{\downarrow}^{\dagger}\right)-\mu\left(c_{\uparrow}^{\dagger} c_{\uparrow}+c_{\downarrow}^{\dagger} c_{\downarrow}\right) \\
& =J\left(\frac{1}{2}\left(S^{+} c_{\downarrow}^{\dagger} c_{\uparrow}+S^{-} c_{\uparrow}^{\dagger} c_{\downarrow}\right)+S^{z}\left(n_{\uparrow}-n_{\downarrow}\right)\right)+\Delta\left(c_{\uparrow} c_{\downarrow}+c_{\downarrow}^{\dagger} c_{\uparrow}^{\dagger}\right)-\mu\left(n_{\uparrow}+n_{\downarrow}\right)
\end{aligned}
$$

Diagonalization of $\mathcal{H}_{\mathrm{SK}}$ now provides the ground state, which at $\mu=0=\Delta$ is the Kondo singlet

$$
|K S\rangle=\frac{1}{\sqrt{2}}(|\Downarrow \uparrow\rangle-|\Uparrow \downarrow\rangle) .
$$

In the left panel of figure F.1 it can be seen that sufficiently strong $\Delta$ leads to a change in the ground state. The degenerate ground state amounts to an antisymmetrized combination of an empty and a doubly occupied conduction site:

$$
\begin{aligned}
\left|G S_{\Delta_{1}}\right\rangle & =\frac{1}{\sqrt{2}}(|\Uparrow\rangle-|\Uparrow \uparrow \downarrow\rangle) \\
\left|G S_{\Delta_{2}}\right\rangle & =\frac{1}{\sqrt{2}}(|\Downarrow\rangle-|\Downarrow \uparrow \downarrow\rangle)
\end{aligned}
$$

These ground states can be distinguished by looking at $\left\langle S^{2}\right\rangle$. Within VCA it is difficult to directly access $\langle\mathbf{S}\rangle$, but $\langle\mathbf{S} \cdot \mathbf{s}\rangle$ can be considered instead. In the case of the Kondo singlet, where

this leads to

$$
\langle\mathbf{S} \cdot \mathbf{s}\rangle=-\frac{3}{4},
$$

$$
\left\langle S^{2}\right\rangle_{|K S\rangle}=2\langle\mathbf{S} \cdot \mathbf{s}\rangle+\mathbf{S}^{2}+\mathbf{s}^{2}=2 \cdot\left(-\frac{3}{4}\right)+\frac{3}{4}+\frac{3}{4}=0 .
$$


For the degenerate ground states $\left|G S_{\Delta_{1 / 2}}\right\rangle$ one gets

$$
\langle\mathbf{S} \cdot \mathbf{s}\rangle_{\left|G S_{\Delta_{1 / 2}}\right\rangle}=0 .
$$

The necessary value $\Delta_{c}$ to arrive at this degenerate ground state is plotted in the right panel of figure F.1 as a function of chemical potential $\mu$.
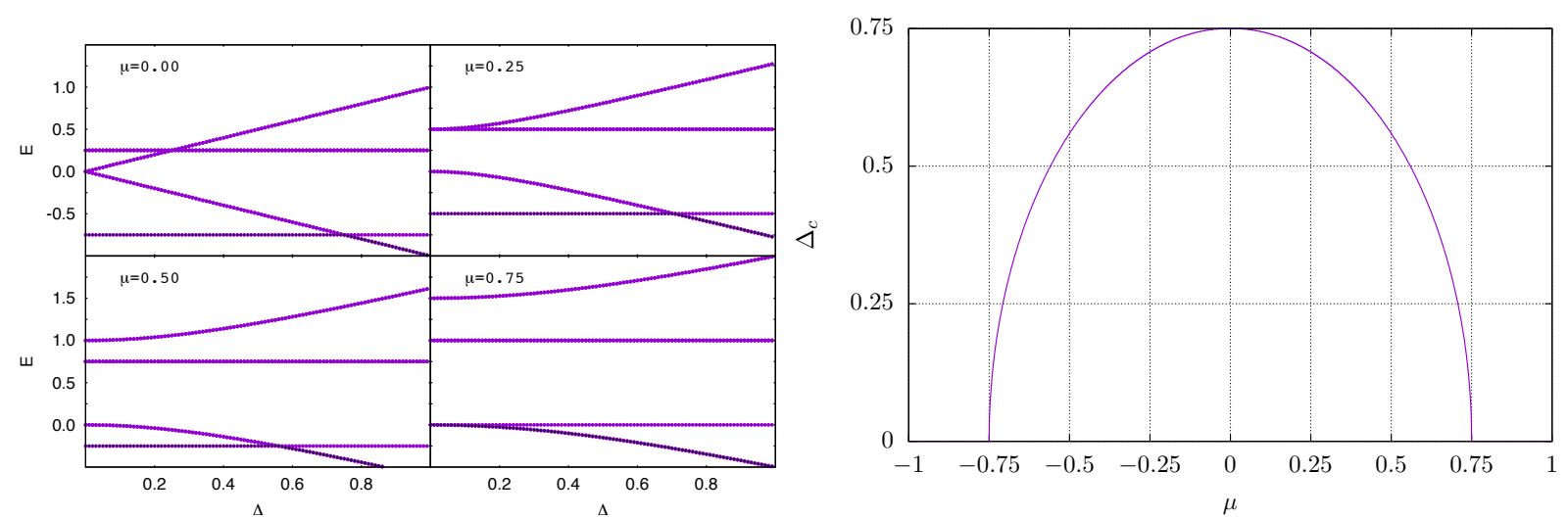

Figure F.1: Left panel: Energy spectrum of a single Kondo site as a function of pairing strength $\Delta$ for different chemical potentials $\mu$. The Kondo exchange is set to $J=1$. Right panel: Critical pairing strength $D_{c}$ at which the Kondo singlet $|K S\rangle$ is replaced by a degenerate ground state $\left|G S_{\Delta_{1 / 2}}\right\rangle$, details see text.

This considerations are important for the Kondo lattice model in the atomic limit $J / t \rightarrow$ $\infty$, where the physics is dominated by the local spin-spin coupling. For large $J / t \gg 1$ the hopping can be regarded as a perturbation which means that we still expect a behavior of the system similar to the situation in the previous subsection.

In any case, the trivial "superconductor" is only realized, if the pairing strength $\Delta$ acquires a sufficiently large value to replace the Kondo singlet as ground state, i.e. $\Delta>3 J / 4$.

\section{\begin{tabular}{l|l} 
An Isolated $2 \times 2$ Cluster & F.2
\end{tabular}}

The next step is to investigate the energy spectrum of a $2 \times 2$ cluster to include the effect of hopping and short-range correlations in the considerations of the previous subsection. Again, for small values of the pairing field $\Delta$, the system has a well defined ground state separated from the rest of the spectrum (see figure F.2). At $\Delta=0$ the second lowest eigenenergy $E_{1}$ is 4 -fold degenerate and the third lowest energy level even 8 -fold. This 8 -fold degenerate level splits up into two 4-fold degenerate levels if one turns on a small pairing $\Delta$. The lower of the split up energy levels is referred to as $E_{2}$.

At half-filling one expects to find a Kondo insulator, which means that all conduction electrons are bound into singlets with the local magnetic moments. A superconducting ground state requires free conduction electrons, which means that the amount of Kondo singlets has to be at least reduced. Besides determination of the superconducting anomalous expectation value $\left\langle c_{\uparrow} c_{\downarrow}\right\rangle$ it is hence essential to determine the expectation value of the spin-spin correlator in order to gain some insights into the ground state properties. Unfortunately, within VCA one is quite limited in the observables that are directly accessible, which are those whose correlator can be connected to the electric Green function. 
This means that the spin-spin correlator between local moments and conduction electrons is not directly accessible as the Green function does not include spin operators of the local moments.

Nonetheless, a way out exists in the form of a functional derivative of the grand potential with respect to the Kondo exchange $J$ :

$$
\left\langle\mathbf{S}_{\mathbf{i}} \cdot \mathbf{S}_{\mathbf{i}}\right\rangle=\frac{\partial \Omega}{\partial J}
$$

This spin-spin correlator is calculated as a numerical derivative with step width $\Delta J=$ 0.01 , see the lowest panel of figure F.2.

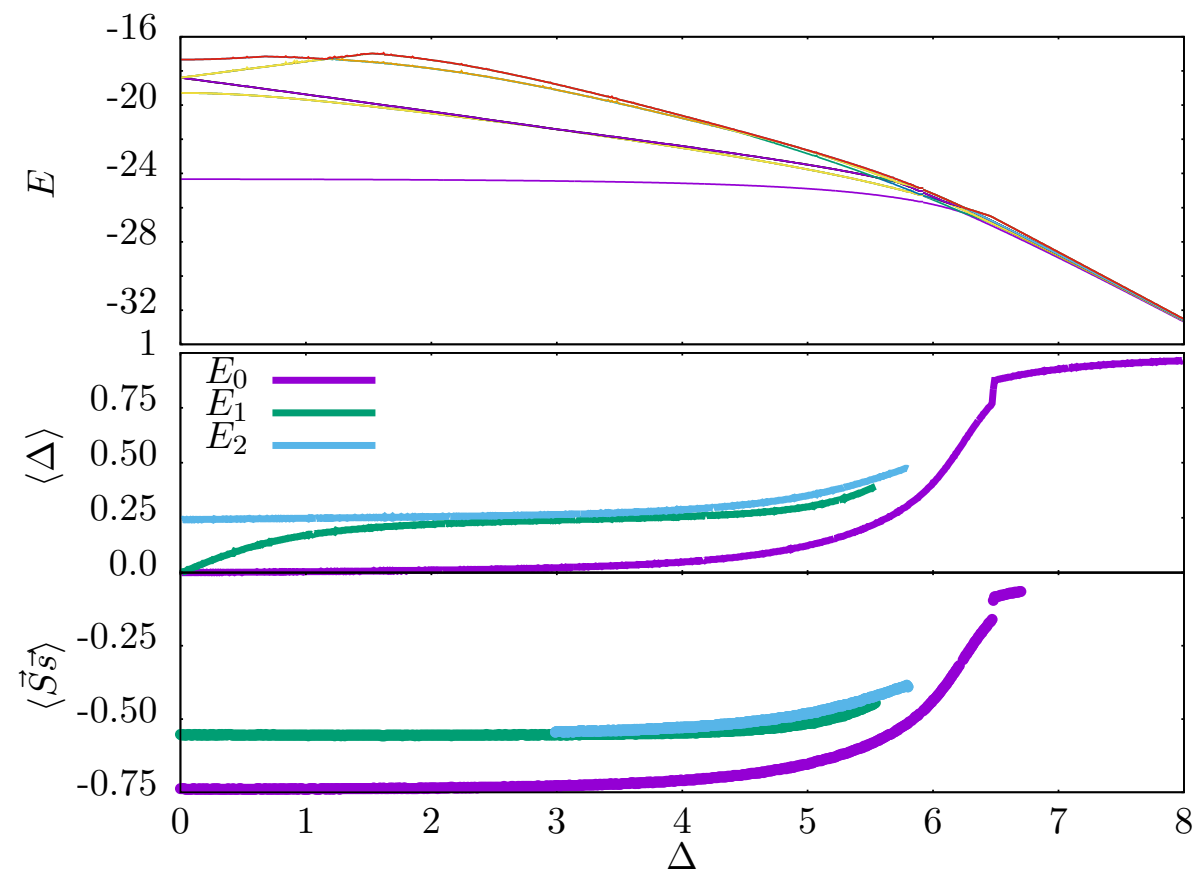

Figure F.2: Lowest 15 energies (top), the superconducting expectation value (middle) and the spin-spin correlator (bottom) of a $2 \times 2$ cluster with open boundary conditions as a function of pairing strength $\Delta$ at $J / t=8.0$. Most of the energies are degenerate. Due to multiple energy level crossings, the derivatives are not shown for $\Delta>5.8$ in the lower plot.

For small $\Delta$ the system's ground state is the Kondo singlet, which is characterized by a spin-spin correlator of $\langle\mathbf{S} \cdot \sigma\rangle=-3 / 4$ and zero superconducting expectation value.

However, compared to the single Kondo site case from section $\mathrm{F}$ the situation here is more complicated as there are various energy level crossings. For example, at $\Delta / J=2.97$ the levels $E_{1}$ and $E_{2}$ cross. In figure F.2 the lowest three energy levels are always labeled with $E_{0}-E_{2}$. A problem occurs at $\Delta / J \approx 6$ where many energy levels come down to cross the former ground state energy $E_{0}$. Therefore it is very difficult to follow the "Kondo singlet" as one would need to include way more than 15 eigenstates in the Band Lanczos algorithm in order to still follow the eigenstate for larger $\Delta$. One of the side effects of this problem shows up in a discontinuity in $\langle\Delta\rangle=\partial E_{0} / \partial \Delta$ and $\left\langle c_{\uparrow} c_{\downarrow}\right\rangle=\partial E_{0} / \partial J$ as suddenly another eigenstate has the lowest eigenenergy. Albeit this problem, the data is sufficient to show that the former Kondo singlet eigenstate more and more deviates even before being finally overtaken energetically by one of the "pure" superconducting eigenstates. Looking at the conduction electron density $n=\partial E_{0} / \partial \mu$ reveals, that the Kondo singlet 
ground state is half-filled, whereas the superconducting eigenstates of $E_{1}$ and $E_{2}$ have a density of $n=1.25$ which means that they are from another quantum sector which becomes energetically preferable at large $\Delta$. 


\section{Bibliography}

[AALR79] E. Abrahams, P. W. Anderson, D. C. Licciardello, and T. V. Ramakrishnan. Scaling Theory of Localization: Absence of Quantum Diffusion in Two Dimensions. Phys. Rev. Lett. 42, 673 (1979).

[AAPH06a] M. Aichhorn, E. Arrigoni, M. Potthoff, and W. Hanke. Antiferromagnetic to superconducting phase transition in the hole- and electron-doped Hubbard model at zero temperature. Phys. Rev. B 74, 024508 (2006).

[AAPH06b] M. Aichhorn, E. Arrigoni, M. Potthoff, and W. Hanke. Variational cluster approach to the Hubbard model: Phase-separation tendency and finite-size effects. Phys. Rev. B 74, 235117 (2006).

$\left[\mathrm{ABB}^{+} 89\right]$ G. Aeppli, D. Bishop, C. Broholm, E. Bucher, K. Siemensmeyer, M. Steiner, and N. Stüsser. Magnetic order in the different superconducting states of $\mathrm{UPt}_{3}$. Phys. Rev. Lett. 63, 676 (1989).

[ABDV $\left.{ }^{+} 06\right]$ C. H. Ahn, A. Bhattacharya, M. Di Ventra, J. N. Eckstein, C. D. Frisbie, M. E. Gershenson, A. M. Goldman, I. H. Inoue, J. Mannhart, A. J. Millis, A. F. Morpurgo, D. Natelson, and J.-M. Triscone. Electrostatic modification of novel materials. Rev. Mod. Phys. 78, 1185 (2006).

[ABF13] M. Z. Asadzadeh, F. Becca, and M. Fabrizio. Variational Monte Carlo approach to the two-dimensional Kondo lattice model. Phys. Rev. B 87, 205144 (2013).

[ADEvdL03] M. Aichhorn, M. Daghofer, H. G. Evertz, and W. von der Linden. Lowtemperature Lanczos method for strongly correlated systems. Phys. Rev. B 67, 161103 (2003).

[AFB14] M. Z. Asadzadeh, M. Fabrizio, and F. Becca. Superconductivity from spoiling magnetism in the Kondo lattice model. Phys. Rev. B 90, 205113 (2014).

[AG61] A. Abrikosov and L. Gor'kov. Contribution to the Theory of Superconducting Alloys with Paramagnetic Impurities. Sov. Phys. JETP 12, 1243 (1961).

[AGO75] K. Andres, J. E. Graebner, and H. R. Ott. 4f-Virtual-Bound-State Formation in $\mathrm{CeAl}_{3}$ at Low Temperatures. Phys. Rev. Lett. 35, 1779 (1975).

[AI96] F. F. Assaad and M. Imada. Insulator-Metal Transition in the One- and Two-Dimensional Hubbard Models. Phys. Rev. Lett. 76, 3176 (1996).

[AJGH16] M. Abdel-Jawad, E. Gati, and B. Hartmann. Private Communication at the Gordon Research Seminar on Conductivity and Magnetism in Molecular Materials (2016).

[AJKW $\left.{ }^{+} 15\right]$ M. Abdel-Jawad, R. Kato, I. Watanabe, N. Tajima, and Y. Ishii. Universality Class of the Mott Transition. Phys. Rev. Lett. 114, 106401 (2015).

[AM76] N. W. Ashcroft and D. Mermin. Solid State Physics. Holt, Rinehart and Winston (1976). 
[And81] P. W. Anderson. Valence Fluctuations in Solids: Santa Barbara Institute for Theoretical Physics Conferences, Santa Barbara, California, January 27-30, 1981, chapter Summary Talk, pages 451-457. North Holland Publishing Company (1981).

[APMH94] F. Assaad, R. Preuss, A. Muramatsu, and W. Hanke. Quantum Monte Carlo simulations of Hubbard type models. Journal of Low Temperature Physics 95, 251 (1994).

$\left[\mathrm{ARC}^{+} 16\right]$ S. Acheche, A. Reymbaut, M. Charlebois, D. Sénéchal, and A.-M. S. Tremblay. Mott transition and magnetism on the anisotropic triangular lattice. E-print arXiv:1608.06640 (2016).

[Ass99] F. F. Assaad. Quantum Monte Carlo Simulations of the Half-Filled TwoDimensional Kondo Lattice Model. Phys. Rev. Lett. 83, 796 (1999).

[BA12] M. Bercx and F. F. Assaad. Metamagnetism and Lifshitz transitions in models for heavy fermions. Phys. Rev. B 86, 075108 (2012).

[Bal08] M. Balzer. Füllungs- und wechselwirkungsabhängiger Mott-Übergang Quanten-Cluster-Rechnungen im Rahmen der SelbstenergiefunktionalTheorie. Ph.D. thesis, Universität Würzburg, Am Hubland, 97074 Würzburg (2008).

[Bay62] G. Baym. Self-Consistent Approximations in Many-Body Systems. Phys. Rev. 127, 1391 (1962).

[BCS57] J. Bardeen, L. N. Cooper, and J. R. Schrieffer. Microscopic Theory of Superconductivity. Phys. Rev. 106, 162 (1957).

[BdSL10] L. Bartosch, M. de Souza, and M. Lang. Scaling Theory of the Mott Transition and Breakdown of the Grüneisen Scaling Near a Finite-Temperature Critical End Point. Phys. Rev. Lett. 104, 245701 (2010).

[BDZ08] I. Bloch, J. Dalibard, and W. Zwerger. Many-body physics with ultracold gases. Reviews of Modern Physics 80(3), 885 (2008).

[Bea04] K. S. D. Beach. Identifying the maximum entropy method as a special limit of stochastic analytic continuation. eprint arXiv:cond-mat/0403055 (2004).

[BEG91a] J. Berntsen, T. O. E., and A. Genz. Algorithm 698: DCUHRE: an adaptive multidemensional integration routine for a vector of integrals. ACM Trans. Math. Softw. 17(4), 452 (1991).

[BEG91b] J. Berntsen, T. O. Espelid, and A. Genz. An adaptive algorithm for the approximate calculation of multiple integrals. ACM Trans. Math. Softw. $\mathbf{1 7}(4), 437$ (1991).

[Bet31] H. Bethe. Zur Theorie der Metalle. Zeitschrift für Physik 71(3), 205 (1931).

$\left[\mathrm{BFH}^{+} 02\right]$ E. D. Bauer, N. A. Frederick, P.-C. Ho, V. S. Zapf, and M. B. Maple. Superconductivity and heavy fermion behavior in $\mathrm{PrOs}_{4} \mathrm{Sb}_{12}$. Phys. Rev. B 65, 100506 (2002).

[BGBG06] C. Berthod, T. Giamarchi, S. Biermann, and A. Georges. Breakup of the Fermi Surface Near the Mott Transition in Low-Dimensional Systems. Phys. Rev. Lett. 97, 136401 (2006).

[BGLS03] J. F. Bonnans, J. C. Gilbert, C. Lemaréchal, and C. A. Sagastizábal. Numerical Optimization, chapter 4.4. Springer, Berlin, Heidelberg, New York (2003). 
[BHP08] M. Balzer, W. Hanke, and M. Potthoff. Mott transition in one dimension: Benchmarking dynamical cluster approaches. Phys. Rev. B 77, 045133 (2008).

[BHV09] K. Byczuk, W. Hofstetter, and D. Vollhardt. Competition between Anderson Localization and Antiferromagnetism in Correlated Lattice Fermion Systems with Disorder. Phys. Rev. Lett. 102, 146403 (2009).

[BK61] G. Baym and L. P. Kadanoff. Conservation Laws and Correlation Functions. Phys. Rev. 124, 287 (1961).

[BKS $\left.{ }^{+} 09\right]$ M. Balzer, B. Kyung, D. Sénéchal, A.-M. S. Tremblay, and M. Potthoff. First-order Mott transition at zero temperature in two dimensions: Variational plaquette study. EPL (Europhysics Letters) 85(1), 17002 (2009).

[Blo08] I. Bloch. Quantum gases. Science 319(5867), 1202 (2008).

[Blu01] S. Blundell. Magnetism in condensed matter. Oxford University Press, Oxford (2001).

[BM86] J. G. Bednorz and K. A. Müller. Possible high $T_{c}$ superconductivity in the Ba-La-Cu-O system. Zeitschrift fur Physik B Condensed Matter 64, 189 (1986).

[Bod13] O. Bodensiek. Superconductivity and Antiferromagnetism in the KondoLattice Model. Ph.D. thesis, Georg-August-Universität Göttingen (2013).

[Bod16] O. Bodensiek (2016). Private communication.

[BP10] M. Balzer and M. Potthoff. Variational cluster approach to ferromagnetism in infinite dimensions and in one-dimensional chains. Phys. Rev. B 82, 174441 (2010).

[Bre73] R. P. Brent. Algorithms for minimization without derivatives. Englewood Cliffs, NJ : Prentice-Hall (1973).

[BSW89] N. E. Bickers, D. J. Scalapino, and S. R. White. Conserving Approximations for Strongly Correlated Electron Systems: Bethe-Salpeter Equation and Dynamics for the Two-Dimensional Hubbard Model. Phys. Rev. Lett. 62, 961 (1989).

[BW13] R. Beyer and J. Wosnitza. Emerging evidence for FFLO states in layered organic superconductors (Review Article). Low Temperature Physics 39(3), 225 (2013).

[BŽPP11] O. Bodensiek, R. Žitko, R. Peters, and T. Pruschke. Low-energy properties of the Kondo lattice model. Journal of Physics: Condensed Matter 23(9), 094212 (2011).

[BŽV $\left.{ }^{+} 13\right]$ O. Bodensiek, R. Žitko, M. Vojta, M. Jarrell, and T. Pruschke. Unconventional Superconductivity from Local Spin Fluctuations in the Kondo Lattice. Phys. Rev. Lett. 110, 146406 (2013).

[CA01] S. Capponi and F. F. Assaad. Spin and charge dynamics of the ferromagnetic and antiferromagnetic two-dimensional half-filled Kondo lattice model. Phys. Rev. B 63, 155114 (2001).

[CCFR79] C. Castellani, C. D. Castro, D. Feinberg, and J. Ranninger. New Model Hamiltonian for the Metal-Insulator Transition. Phys. Rev. Lett. 43, 1957 (1979).

$\left[\mathrm{CCK}^{+} 04\right]$ M. Capone, M. Civelli, S. S. Kancharla, C. Castellani, and G. Kotliar. Cluster-dynamical mean-field theory of the density-driven Mott transition 
in the one-dimensional Hubbard model. Phys. Rev. B 69, 195105 (2004).

[CEVV10] P. Corboz, G. Evenbly, F. Verstraete, and G. Vidal. Simulation of interacting fermions with entanglement renormalization. Phys. Rev. A 81, 010303 (2010).

$\left[\mathrm{CGW}^{+} 03\right]$ J. Custers, P. Gegenwart, H. Wilhelm, K. Neumaier, Y. Tokiwa, O. Trovarelli, C. Geibel, F. Steglich, C. Pepin, and P. Coleman. The break-up of heavy electrons at a quantum critical point. Nature 424(6948), 524 (2003).

[CK94] M. Caffarel and W. Krauth. Exact diagonalization approach to correlated fermions in infinite dimensions: Mott transition and superconductivity. Phys. Rev. Lett. 72, 1545 (1994).

[CKSW12] J.-S. Caux, H. Konno, M. Sorrell, and R. Weston. Exact form-factor results for the longitudinal structure factor of the massless XXZ model in zero field. Journal of Statistical Mechanics: Theory and Experiment 2012(01), P01007 (2012).

[COBV10] P. Corboz, R. Orús, B. Bauer, and G. Vidal. Simulation of strongly correlated fermions in two spatial dimensions with fermionic projected entangledpair states. Phys. Rev. B 81, 165104 (2010).

[Col07] P. Coleman. Heavy Fermions: Electrons at the Edge of Magnetism. John Wiley \& Sons, Ltd (2007).

[Col15a] P. Coleman. Introduction to Many-Body Physics. Cambridge Univ. Press (2015).

[Col15b] P. Coleman. Lecture notes of the Autumn School on Correlated Electrons: Many-Body Physics: From Kondo to Hubbard, chapter Heavy Fermions and the Kondo Lattice. Forschungszentrum Juelich (2015).

[Cor16] P. Corboz. Improved energy extrapolation with infinite projected entangledpair states applied to the two-dimensional Hubbard model. Phys. Rev. B 93, 045116 (2016).

[CPSR01] P. Coleman, C. Pépin, Q. Si, and R. Ramazashvili. How do Fermi liquids get heavy and die? Journal of Physics: Condensed Matter 13(35), R723 (2001).

[CV09] P. Corboz and G. Vidal. Fermionic multiscale entanglement renormalization ansatz. Phys. Rev. B 80, 165129 (2009).

[Dag94] E. Dagotto. Correlated electrons in high-temperature superconductors. Rev. Mod. Phys. 66, 763 (1994).

$\left[\mathrm{DAH}^{+} 04\right]$ C. Dahnken, M. Aichhorn, W. Hanke, E. Arrigoni, and M. Potthoff. Variational cluster approach to spontaneous symmetry breaking: The itinerant antiferromagnet in two dimensions. Phys. Rev. B 70, 245110 (2004).

[dCP62] J. des Cloizeaux and J. J. Pearson. Spin-Wave Spectrum of the Antiferromagnetic Linear Chain. Phys. Rev. 128, 2131 (1962).

[DG02] M. Dressel and G. Grüner. Electrodynamics of Solids Optical Properties of Electrons in Matter. Cambridge Univ. Press (2002).

[dHdBvdB34] W. de Haas, J. de Boer, and G. van den Berg. The electrical resistance of gold, copper and lead at low temperatures. Physica 1(7), 1115 (1934).

[DLacP ${ }^{+}$16] M. Dressel, P. Lazić, A. Pustogow, E. Zhukova, B. Gorshunov, J. A. Schlueter, O. Milat, B. Gumhalter, and S. Tomić. Lattice vibrations of 
the charge-transfer salt $\kappa-(B E D T-T T F)_{2} \mathrm{Cu}_{2}(\mathrm{CN})_{3}$ : Comprehensive explanation of the electrodynamic response in a spin-liquid compound. Phys. Rev. B 93, 081201 (2016).

[DMS12] S. Depenbrock, I. P. McCulloch, and U. Schollwöck. Nature of the SpinLiquid Ground State of the $S=1 / 2$ Heisenberg Model on the Kagome Lattice. Phys. Rev. Lett. 109, 067201 (2012).

[Don77] S. Doniach. The Kondo lattice and weak antiferromagnetism. Physica B $+\mathrm{C}$ 91(0), 231 (1977).

[dSBS $\left.{ }^{+} 07\right]$ M. de Souza, A. Brühl, C. Strack, B. Wolf, D. Schweitzer, and M. Lang. Anomalous Lattice Response at the Mott Transition in a Quasi-2D Organic Conductor. Phys. Rev. Lett. 99, 037003 (2007).

[DXC $\left.{ }^{+} 15\right]$ H. T. Dang, X. Y. Xu, K.-S. Chen, Z. Y. Meng, and S. Wessel. Mott transition in the triangular lattice Hubbard model: A dynamical cluster approximation study. Phys. Rev. B 91, 155101 (2015).

[Eco90] E. N. Economou. Green's functions in quantum physics. Springer (1990).

[Ede14] R. Eder. Comment on 'Non-existence of the Luttinger-Ward functional and misleading convergence of skeleton diagrammatic series for Hubbardlike models'. E-print arXiv:1407.6599 (2014).

[Ede15] R. Eder. Electronic structure of NiO: Antiferromagnetic transition and photoelectron spectra in the ordered phase. Phys. Rev. B 91, 245146 (2015).

[EF63] J. W. Essam and M. E. Fisher. Padé Approximant Studies of the Lattice Gas and Ising Ferromagnet below the Critical Point. The Journal of Chemical Physics 38(4), 802 (1963).

$\left[\mathrm{EFK}^{+} 05\right]$ F. Essler, H. Frahm, A. Klümper, F. Göhmann, and V. E. Korepin. The One-Dimensional Hubbard Model. Cambridge Univ. Press (2005).

[ESLN15] G. Ehlers, J. Sólyom, O. Legeza, and R. M. Noack. Entanglement structure of the Hubbard model in momentum space. Phys. Rev. B 92, 235116 (2015).

[ET02] F. H. L. Essler and A. M. Tsvelik. Weakly coupled one-dimensional Mott insulators. Phys. Rev. B 65, 115117 (2002).

[ET05] F. H. L. Essler and A. M. Tsvelik. Theory of hybrid state in a metal with a small Fermi surface and strong collective excitations. Phys. Rev. B 71, 195116 (2005).

[FAI96] N. Furukawa, F. F. Assaad, and M. Imada. Critical Exponents of the MetalInsulator Transition in the Two-Dimensional Hubbard Model. Journal of the Physical Society of Japan 65(7), 2339 (1996).

[FFdBM83] P. Frings, J. Franse, F. de Boer, and A. Menovsky. Magnetic properties of $U_{x} P t_{y}$ compounds. Journal of Magnetism and Magnetic Materials 31, 240 (1983).

[FMI $\left.{ }^{+} 15\right]$ T. Furukawa, K. Miyagawa, T. Itou, M. Ito, H. Taniguchi, M. Saito, S. Iguchi, T. Sasaki, and K. Kanoda. Quantum Spin Liquid Emerging from Antiferromagnetic Order by Introducing Disorder. Phys. Rev. Lett. 115, 077001 (2015).

$\left[\mathrm{FMT}^{+} 15\right]$ T. Furukawa, K. Miyagawa, H. Taniguchi, R. Kato, and K. Kanoda. Quantum criticality of Mott transition in organic materials. Nat Phys 11(3), 221 (2015). 
[FP10] S. Filor and T. Pruschke. A self-energy functional approach for spin systems. Journal of Physics: Conference Series 200(2), 022007 (2010).

[FP14] S. Filor and T. Pruschke. Variational cluster approximation to the thermodynamics of quantum spin systems. New Journal of Physics 16(6), 063059 (2014).

[Fre00] R. Freund. Templates for the solution of algebraic eigenvalue problems: A practical guide, chapter Band Lanczos Method (Section 4.6). SIAM (2000).

[FW03] A. L. Fetter and J. D. Walecka. Quantum Theory of Many-Particle Systems. Dover, New York (2003).

$\left[\mathrm{FWB}^{+}\right.$09] S. Friedemann, T. Westerkamp, M. Brando, N. Oeschler, S. Wirth, P. Gegenwart, C. Krellner, C. Geibel, and F. Steglich. Detaching the antiferromagnetic quantum critical point from the Fermi-surface reconstruction in YbRh2Si2. Nat Phys 5(7), 465 (2009).

[GAJV16] D. Guterding, M. Altmeyer, H. O. Jeschke, and R. Valentí. Neardegeneracy of extended $s+d_{x^{2}-y^{2}}$ and $d_{x y}$ order parameters in quasi-twodimensional organic superconductors. Phys. Rev. B 94, 024515 (2016).

[Geb03] F. Gebhard. The mott metal-insulator transition: models and methods, volume 137 of Springer Tracts in Modern Physics. Springer (2003).

[Gez16] R. Gezzi. Private communication (2016).

[GFC04] A. Georges, S. Florens, and T. A. Costi. The Mott transition: Unconventional transport, spectral weight transfers, and critical behaviour. J. Phys. IV France 114, 165 (2004).

[GGM $\left.{ }^{+} 16\right]$ E. Gati, M. Garst, R. S. Manna, U. Tutsch, B. Wolf, D. Zielke, L. Bartosch, H. Schubert, T. Sasaki, J. A. Schlueter, and M. Lang. Breakdown of Hooke's law of elasticity around the Mott critical endpoint in the organic chargetransfer salt $\kappa-(B E D T-T T F)_{2} \mathrm{Cu} / \mathrm{N}(\mathrm{CN})_{2} / \mathrm{Cl}(2016)$. GRC on Conductivity and Mangetism in Molecular Materials.

[Gia97] T. Giamarchi. Mott transition in one dimension. Physica B: Condensed Matter 230-232, 975 (1997).

$\left[\mathrm{GJ}^{+}\right.$10] G. Guennebaud, B. Jacob, et al. Eigen v3. http://eigen.tuxfamily.org (2010).

[Gol92] N. Goldenfeld. Lectures on Phase Transitions and the Renormalization Group. Westview Press (1992).

[Gor58] L. P. Gor'kov. On the Energy Spectrum of Superconductors. Soviet Physics JETP 34(3), 505 (1958).

[GSGB15] P. Gegenwart, F. Steglich, C. Geibel, and M. Brando. Novel types of quantum criticality in heavy-fermion systems. The European Physical Journal Special Topics 224(6), 975 (2015).

[GSS08] P. Gegenwart, Q. Si, and F. Steglich. Quantum criticality in heavy-fermion metals. Nat Phys 4(3), 186 (2008).

[GV93] C. Gros and R. Valentí. Cluster expansion for the self-energy: A simple many-body method for interpreting the photoemission spectra of correlated Fermi systems. Phys. Rev. B 48, 418 (1993).

[Hah05] T. Hahn. Cuba - a library for multidimensional numerical integration. Computer Physics Communications 168(2), 78 (2005). 
[Hah06] T. Hahn. The Cuba library. Nuclear Instruments and Methods in Physics Research Section A: Accelerators, Spectrometers, Detectors and Associated Equipment 559(1), 273 (2006).

[HEP16] F. Hofmann, M. Eckstein, and M. Potthoff. Nonequilibrium self-energy functional approach to the dynamical Mott transition. Phys. Rev. B 93, 235104 (2016).

[Hew93] A. C. Hewson. The Kondo problem to heavy fermions, volume 2. Cambridge university press (1993).

$\left[\mathrm{HGdN}^{+} 07\right]$ N. T. Huy, A. Gasparini, D. E. de Nijs, Y. Huang, J. C. P. Klaasse, T. Gortenmulder, A. de Visser, A. Hamann, T. Görlach, and H. v. Löhneysen. Superconductivity on the Border of Weak Itinerant Ferromagnetism in UCoGe. Phys. Rev. Lett. 99, 067006 (2007).

[HK13] S. Hoshino and Y. Kuramoto. Itinerant Versus Localized Heavy-Electron Magnetism. Phys. Rev. Lett. 111, 026401 (2013).

[HKS12] O. Howczak, J. Kaczmarczyk, and J. Spałek. From magnetic and Kondocompensated states to unconventional superconductivity in heavy fermions: a unified approach. E-print arXiv:1209.0621 (2012).

[HMJK00] M. H. Hettler, M. Mukherjee, M. Jarrell, and H. R. Krishnamurthy. Dynamical cluster approximation: Nonlocal dynamics of correlated electron systems. Phys. Rev. B 61, 12739 (2000).

[HMS14] B. Hartmann, J. Müller, and T. Sasaki. Mott metal-insulator transition induced by utilizing a glasslike structural ordering in low-dimensional molecular conductors. Phys. Rev. B 90, 195150 (2014).

[Hub63] J. Hubbard. Electron correlations in narrow energy bands. Proceedings of the Royal Society of London. Series A. Mathematical and Physical Sciences 276(1365), 238 (1963).

[HV98] W. Hofstetter and D. Vollhardt. Frustration of antiferromagnetism in the t-t'-Hubbard model at weak coupling. Annalen der Physik 510(1), 48 (1998).

$\left[\mathrm{HZP}^{+} 15\right]$ B. Hartmann, D. Zielke, J. Polzin, T. Sasaki, and J. Müller. Critical Slowing Down of the Charge Carrier Dynamics at the Mott Metal-Insulator Transition. Phys. Rev. Lett. 114, 216403 (2015).

[IFT98] M. Imada, A. Fujimori, and Y. Tokura. Metal-insulator transitions. Rev. Mod. Phys. 70, 1039 (1998).

[Ima04] M. Imada. Quantum Mott Transition and Multi-Furcating Criticality. Journal of the Physical Society of Japan 73(7), 1851 (2004).

[Ima05] M. Imada. Universality classes of metal-insulator transitions in strongly correlated electron systems and mechanism of high-temperature superconductivity. Phys. Rev. B 72, 075113 (2005).

[IMY10] M. Imada, T. Misawa, and Y. Yamaji. Unconventional quantum criticality emerging as a new common language of transition-metal compounds, heavyfermion systems, and organic conductors. Journal of Physics: Condensed Matter 22(16), 164206 (2010).

[IS88] Y. A. Izyumov and Y. N. Skryabin. Statistical Mechanics of Magnetically Ordered Substances (1988).

[JG89] R. O. Jones and O. Gunnarsson. The density functional formalism, its applications and prospects. Rev. Mod. Phys. 61, 689 (1989). 
[Joh] S. G. Johnson. The NLopt nonlinear-optimization package. Http://abinitio.mit.edu/nlopt.

[JP93] M. T. Jones and M. L. Patrick. The Lanczos algorithm for the generalized symmetric eigenproblem on shared-memory architectures. Applied Numerical Mathematics 12(5), 377 (1993).

[JP94] J. Jaklič and P. Prelovšek. Lanczos method for the calculation of finitetemperature quantities in correlated systems. Phys. Rev. B 49, 5065 (1994).

[Kas56] T. Kasuya. A Theory of Metallic Ferro- and Antiferromagnetism on Zener's Model. Progress of Theoretical Physics 16(1), 45 (1956).

[Kat04] R. Kato. Conducting Metal Dithiolene Complexes: Structural and Electronic Properties. Chemical Reviews 104(11), 5319 (2004).

[KB06] A. A. Kordyuk and S. V. Borisenko. ARPES on high-temperature superconductors: Simplicity vs. complexity. Low Temp. Phys. 32, 298 (2006).

[KC15] J. Knolle and N. R. Cooper. Quantum Oscillations without a Fermi Surface and the Anomalous de Haas-van Alphen Effect. Phys. Rev. Lett. 115, 146401 (2015).

[KFG15] E. Kozik, M. Ferrero, and A. Georges. Nonexistence of the Luttinger-Ward Functional and Misleading Convergence of Skeleton Diagrammatic Series for Hubbard-Like Models. Phys. Rev. Lett. 114, 156402 (2015).

$\left[\mathrm{KGH}^{+} 67\right]$ L. P. Kadanoff, W. Götze, D. Hamblen, R. Hecht, E. A. S. Lewis, V. V. Palciauskas, M. Rayl, J. Swift, D. Aspnes, and J. Kane. Static Phenomena Near Critical Points: Theory and Experiment. Rev. Mod. Phys. 39, 395 (1967).

[KI01] T. Kashima and M. Imada. Magnetic and Metal-Insulator Transitions through Bandwidth Control in Two-Dimensional Hubbard Models with Nearest and Next-Nearest Neighbor Transfers. Journal of the Physical Society of Japan $\mathbf{7 0}(10), 3052$ (2001).

[Kit96] C. Kittel. Introduction to Solid State Physics. Wiley (1996).

[KK98] H. Kino and H. Kontani. Phase Diagram of Superconductivity on the Anisotropic Triangular Lattice Hubbard Model: An Effective Model of $\kappa$ (BEDT-TTF) Salts. Journal of the Physical Society of Japan 67(11), 3691 (1998).

[KK11] K. Kanoda and R. Kato. Mott Physics in Organic Conductors with Triangular Lattices. Annual Review of Condensed Matter Physics 2(1), 167 (2011).

[KLR00] G. Kotliar, E. Lange, and M. J. Rozenberg. Landau Theory of the Finite Temperature Mott Transition. Phys. Rev. Lett. 84, 5180 (2000).

[KM99] H. Kondo and T. Moriya. Origin of Superconductivity in 2D-Organic Compounds and High-T c Cuprates. Journal of the Physical Society of Japan 68(10), 3170 (1999).

[KMCvdB11] A. Klauser, J. Mossel, J.-S. Caux, and J. van den Brink. Spin-Exchange Dynamical Structure Factor of the $S=1 / 2$ Heisenberg Chain. Phys. Rev. Lett. 106, 157205 (2011).

[KMF07] T. Koretsune, Y. Motome, and A. Furusaki. Exact Diagonalization Study of Mott Transition in the Hubbard Model on an Anisotropic Triangular Lattice. Journal of the Physical Society of Japan 76(7), 074719 (2007). 
[KMK97] A. Kawamoto, K. Miyagawa, and K. Kanoda. Deuterated $\kappa-(B E D T-$ $\mathrm{TTF})_{2} \mathrm{Cu}\left[\mathrm{N}(\mathrm{CN})_{2}\right] \mathrm{Br}:$ A system on the border of the superconductormagnetic-insulator transition. Phys. Rev. B 55, 14140 (1997).

[KMK05] F. Kagawa, K. Miyagawa, and K. Kanoda. Unconventional critical behaviour in a quasi-two-dimensional organic conductor. Nature 436(7050), $534(2005)$.

[KMK09] F. Kagawa, K. Miyagawa, and K. Kanoda. Magnetic Mott criticality in a $\kappa$-type organic salt probed by NMR. Nat Phys 5(12), 880 (2009).

[Kon64] J. Kondo. Resistance Minimum in Dilute Magnetic Alloys. Progress of Theoretical Physics 32(1), 37 (1964).

[KSE $\left.{ }^{+} 16\right]$ Y. Kawasugi, K. Seki, Y. Edagawa, Y. Sato, J. Pu, T. Takenobu, S. Yunoki, H. M. Yamamoto, and R. Kato. Electron-hole doping asymmetry of Fermi surface reconstructed in a simple Mott insulator. Nature Communications 7, 12356 (2016).

[KSPB01] G. Kotliar, S. Y. Savrasov, G. Pálsson, and G. Biroli. Cellular Dynamical Mean Field Approach to Strongly Correlated Systems. Phys. Rev. Lett. 87, 186401 (2001).

[KT06] B. Kyung and A.-M. S. Tremblay. Mott Transition, Antiferromagnetism, and d-Wave Superconductivity in Two-Dimensional Organic Conductors. Phys. Rev. Lett. 97, 046402 (2006).

[KV04] G. Kotliar and D. Vollhardt. Strongly Correlated Materials: Insights From Dynamical Mean-Field Theory. Physics Today 57(3), 53 (2004).

[KYRa15] A. Kramida, Yu. Ralchenko, J. Reader, and and NIST ASD Team. NIST Atomic Spectra Database. NIST Atomic Spectra Database (ver. 5.3), [Online]. Available: http://physics.nist.gov/asd [2016, October 6]. National Institute of Standards and Technology, Gaithersburg, MD. (2015).

$\left[\mathrm{KYT}^{+} 09\right]$ Y. Kawasugi, H. M. Yamamoto, N. Tajima, T. Fukunaga, K. Tsukagoshi, and R. Kato. Field-Induced Carrier Delocalization in the Strain-Induced Mott Insulating State of an Organic Superconductor. Phys. Rev. Lett. 103, 116801 (2009).

$\left[\mathrm{LAB}^{+} 15\right]$ J. P. F. LeBlanc, A. E. Antipov, F. Becca, I. W. Bulik, G. K.-L. Chan, C.-M. Chung, Y. Deng, M. Ferrero, T. M. Henderson, C. A. JiménezHoyos, E. Kozik, X.-W. Liu, A. J. Millis, N. V. Prokof'ev, M. Qin, G. E. Scuseria, H. Shi, B. V. Svistunov, L. F. Tocchio, I. S. Tupitsyn, S. R. White, S. Zhang, B.-X. Zheng, Z. Zhu, and E. Gull. Solutions of the TwoDimensional Hubbard Model: Benchmarks and Results from a Wide Range of Numerical Algorithms. Phys. Rev. X 5, 041041 (2015).

[LC79] C. Lacroix and M. Cyrot. Phase diagram of the Kondo lattice. Phys. Rev. B 20, 1969 (1979).

[Leg06] A. J. Leggett. What DO we know about high Tc? Nat Phys 2(3), 134 (2006).

$\left[\mathrm{LGJ}^{+}\right.$03] P. Limelette, A. Georges, D. Jérome, D.rome, P. Wzietek, P. Metcalf, and J. M. Honig. Universality and Critical Behavior at the Mott Transition. Science 302(5642), 89 (2003).

[LIM09] A. Liebsch, H. Ishida, and J. Merino. Mott transition in two-dimensional frustrated compounds. Phys. Rev. B 79, 195108 (2009). 
[LL05] S.-S. Lee and P. A. Lee. U(1) Gauge Theory of the Hubbard Model: Spin Liquid States and Possible Application to $\kappa-(\mathrm{BEDT}-\mathrm{TTF})_{2} \mathrm{Cu}_{2}(\mathrm{CN})_{3}$. Phys. Rev. Lett. 95, 036403 (2005).

[LML87] M. Lavagna, A. J. Millis, and P. A. Lee. d-Wave Superconductivity in the Large-Degeneracy Limit of the Anderson Lattice. Phys. Rev. Lett. 58, 266 (1987).

$\left[\mathrm{LMP}^{+}\right.$16] B. Lenz, S. R. Manmana, T. Pruschke, F. F. Assaad, and M. Raczkowski. Mott Quantum Criticality in the Anisotropic 2D Hubbard Model. Phys. Rev. Lett. 116, 086403 (2016).

[LR14] A. Lavarélo and G. Roux. Spinon excitation spectra of the J1-J2 chain from analytical calculations in the dimer basis and exact diagonalization. The European Physical Journal B 87(10), 229 (2014).

[LS70] A. P. Levanyuk and A. A. Sobyanin. Second-order phase transitions without divergencies in the second derivatives of the thermodynamic potential. Sov. Phys. JETP Lett. 11, 371 (1970). [Zh. Eksp. Teor. Fiz. Pis. Red. 11, 540 (1970)].

[LST05] J. Liu, J. Schmalian, and N. Trivedi. Pairing and Superconductivity Driven by Strong Quasiparticle Renormalization in Two-Dimensional Organic Charge Transfer Salts. Phys. Rev. Lett. 94, 127003 (2005).

$\left[\mathrm{LTP}^{+} 15\right]$ M. Laubach, R. Thomale, C. Platt, W. Hanke, and G. Li. Phase diagram of the Hubbard model on the anisotropic triangular lattice. Phys. Rev. B 91, 245125 (2015).

[Lut60] J. M. Luttinger. Fermi Surface and Some Simple Equilibrium Properties of a System of Interacting Fermions. Phys. Rev. 119, 1153 (1960).

$\left[\mathrm{LVY}^{+} 12\right]$ D. Lu, I. M. Vishik, M. Yi, Y. Chen, R. G. Moore, and Z.-X. Shen. AngleResolved Photoemission Studies of Quantum Materials. Annual Review of Condensed Matter Physics 3(1), 129 (2012).

[LW60] J. M. Luttinger and J. C. Ward. Ground-State Energy of a Many-Fermion System. II. Phys. Rev. 118, 1417 (1960).

[LW68] E. H. Lieb and F. Y. Wu. Absence of Mott Transition in an Exact Solution of the Short-Range, One-Band Model in One Dimension. Phys. Rev. Lett. 20, 1445 (1968).

[MA08] L. C. Martin and F. F. Assaad. Evolution of the Fermi Surface across a Magnetic Order-Disorder Transition in the Two-Dimensional Kondo Lattice Model: A Dynamical Cluster Approach. Phys. Rev. Lett. 101, 066404 (2008).

[Mah90] G. D. Mahan. Many-Particle Physics. Plenum Press, second edition edition (1990).

[Map68] M. Maple. The superconducting transition temperature of $L a_{1-x} G d_{x} A l_{2}$. Physics Letters A 26(10), 513 (1968).

[Mar82] R. M. Martin. Fermi-Surfae Sum Rule and its Consequences for Periodic Kondo and Mixed-Valence Systems. Phys. Rev. Lett. 48, 362 (1982).

[MBA10] L. C. Martin, M. Bercx, and F. F. Assaad. Fermi surface topology of the two-dimensional Kondo lattice model: Dynamical cluster approximation approach. Phys. Rev. B 82, 245105 (2010). 
[MDD $\left.{ }^{+} 08\right]$ J. Merino, M. Dumm, N. Drichko, M. Dressel, and R. H. McKenzie. Quasiparticles at the Verge of Localization near the Mott Metal-Insulator Transition in a Two-Dimensional Material. Phys. Rev. Lett. 100, 086404 (2008).

[MH89] E. Müller-Hartmann. Correlated fermions on a lattice in high dimensions. Zeitschrift für Physik B Condensed Matter 74(4), 507 (1989).

[MI06] T. Mizusaki and M. Imada. Gapless quantum spin liquid, stripe, and antiferromagnetic phases in frustrated Hubbard models in two dimensions. Phys. Rev. B 74, 014421 (2006).

[MI07] T. Misawa and M. Imada. Quantum criticality around metal-insulator transitions of strongly correlated electron systems. Phys. Rev. B 75, 115121 (2007).

[MJPH05] T. Maier, M. Jarrell, T. Pruschke, and M. H. Hettler. Quantum cluster theories. Rev. Mod. Phys. 77, 1027 (2005).

[MP89] H.-D. Meyer and S. Pal. A band-Lanczos method for computing matrix elements of a resolvent. The Journal of Chemical Physics 91(10), 6195 (1989).

[MSRV86] K. Miyake, S. Schmitt-Rink, and C. M. Varma. Spin-fluctuation-mediated even-parity pairing in heavy-fermion superconductors. Phys. Rev. B 34, 6554 (1986).

[MTBB81] G. Müller, H. Thomas, H. Beck, and J. Bonner. Quantum spin dynamics of the antiferromagnetic linear chain in zero and nonzero magnetic field. Phys. Rev. B 24, 1429 (1981).

[MV64] A. Matthiessen and C. Vogt. On the Influence of Temperature on the Electric Conducting-Power of Alloys. Philosophical Transactions of the Royal Society of London 154, 167 (1864).

[MV30a] W. Meissner and B. Voigt. Messungen mit Hilfe von flüssigem Helium XI Widerstand der reinen Metalle in tiefen Temperaturen. Annalen der Physik 399(8), 892 (1930).

[MV30b] W. Meissner and B. Voigt. Messungen mit Hilfe von flüssigem Helium XI Widerstand der reinen Metalle in tiefen Temperaturen. Annalen der Physik 399(7), 761 (1930).

[MV89] W. Metzner and D. Vollhardt. Correlated Lattice Fermions in $d=\infty$ Dimensions. Phys. Rev. Lett. 62, 324 (1989).

[MWI02] H. Morita, S. Watanabe, and M. Imada. Nonmagnetic Insulating States near the Mott Transitions on Lattices with Geometrical Frustration and Implications for $\kappa-(E T)_{2} \mathrm{Cu}_{2}(C N)_{3}$. Journal of the Physical Society of Japan 71(9), 2109 (2002).

[MY13] K. Masuda and D. Yamamoto. Formation of Cooper pairs between conduction and localized electrons in heavy-fermion superconductors. Phys. Rev. B 87, 014516 (2013).

[MY15] K. Masuda and D. Yamamoto. Variational cluster approach to s-wave pairing in heavy-fermion superconductors. Phys. Rev. B 91, 104508 (2015).

[MYI06] T. Misawa, Y. Yamaji, and M. Imada. Quantum Critical 'Opalescence' around Metal-Insulator Transitions. Journal of the Physical Society of Japan 75(8), 083705 (2006). 
[Nam60] Y. Nambu. Quasi-Particles and Gauge Invariance in the Theory of Superconductivity. Phys. Rev. 117, 648 (1960).

[NM65] J. A. Nelder and R. Mead. A Simplex Method for Function Minimization. The Computer Journal 7(4), 308 (1965).

[Nol09] W. Nolting. Grundkurs Theoretische Physik 7 - Viel-Teilchen-Theorie. Springer, 7 edition (2009).

[NSST08] A. H. Nevidomskyy, C. Scheiber, D. Sénéchal, and A.-M. S. Tremblay. Magnetism and d-wave superconductivity on the half-filled square lattice with frustration. Phys. Rev. B 77, 064427 (2008).

$\left[\mathrm{NSW}^{+} 10\right]$ S. Nair, O. Stockert, U. Witte, M. Nicklas, R. Schedler, K. Kiefer, J. D. Thompson, A. D. Bianchi, Z. Fisk, S. Wirth, and F. Steglich. Magnetism and superconductivity driven by identical $4 f$ states in a heavy-fermion metal. PNAS 107(21), 9537 (2010).

[NYI12] K. Nakamura, Y. Yoshimoto, and M. Imada. Ab initio two-dimensional multiband low-energy models of $\mathrm{EtMe}_{3} \mathrm{Sb}\left[\mathrm{Pd}(\mathrm{dmit})_{2}\right]_{2}$ and $\kappa$-(BEDT$\mathrm{TTF})_{2} \mathrm{Cu}(\mathrm{NCS})_{2}$ with comparisons to single-band models. Phys. Rev. B 86, 205117 (2012).

[OBS10] A. Ovchinnikov, I. Bostrem, and V. Sinitsyn. Cluster perturbation theory for spin Hamiltonians. Theoretical and Mathematical Physics 162, 179 (2010).

[OKK09] J. Otsuki, H. Kusunose, and Y. Kuramoto. Evolution of a Large Fermi Surface in the Kondo Lattice. Phys. Rev. Lett. 102, 017202 (2009).

[OMTK08] T. Ohashi, T. Momoi, H. Tsunetsugu, and N. Kawakami. Finite Temperature Mott Transition in Hubbard Model on Anisotropic Triangular Lattice. Phys. Rev. Lett. 100, 076402 (2008).

[ON03] S. Onoda and N. Nagaosa. Mott Transition vs Multicritical Phenomenon of Superconductivity and Antiferromagnetism - Application to $\kappa$-(BEDTTTF $)_{2} X$-. Journal of the Physical Society of Japan 72(10), 2445 (2003).

[Orú14] R. Orús. A practical introduction to tensor networks: Matrix product states and projected entangled pair states. Annals of Physics 349, 117 (2014).

[Osh00] M. Oshikawa. Topological Approach to Luttinger's Theorem and the Fermi Surface of a Kondo Lattice. Phys. Rev. Lett. 84, 3370 (2000).

[Ots15] J. Otsuki. Competing d-Wave and p-Wave Spin-Singlet Superconductivities in the Two-Dimensional Kondo Lattice. Phys. Rev. Lett. 115, 036404 (2015).

[Ovc08] M. Y. Ovchinnikova. The Fermi surface reconstruction in stripe phases of cuprates. JETP Letters 88(9), 620 (2008).

[Ove59] A. W. Overhauser. New Mechanism of Antiferromagnetism. Phys. Rev. Lett. 3, 414 (1959).

$\left[\mathrm{PBB}^{+} 11\right]$ F. L. Pratt, P. J. Baker, S. J. Blundell, T. Lancaster, S. Ohira-Kawamura, C. Baines, Y. Shimizu, K. Kanoda, I. Watanabe, and G. Saito. Magnetic and non-magnetic phases of a quantum spin liquid. Nature 471(7340), 612 (2011).

[PBK04] O. Parcollet, G. Biroli, and G. Kotliar. Cluster Dynamical Mean Field Analysis of the Mott Transition. Phys. Rev. Lett. 92, 226402 (2004).

[Pet14] R. Peters. private communication (2014). 
$\left[\mathrm{PFF}^{+} 08\right]$ S. Papanikolaou, R. M. Fernandes, E. Fradkin, P. W. Phillips, J. Schmalian, and R. Sknepnek. Universality of Liquid-Gas Mott Transitions at Finite Temperatures. Phys. Rev. Lett. 100, 026408 (2008).

[PK15] R. Peters and N. Kawakami. Large and small Fermi-surface spin density waves in the Kondo lattice model. Phys. Rev. B 92, 075103 (2015).

[Pla10] N. Plakida. High-Temperature Cuprate Superconductors: Experiment, Theory, and Applications, volume 166. Springer Science \& Business Media (2010).

[Pot03a] M. Potthoff. Self-energy-functional approach: Analytical results and the Mott-Hubbard transition. The European Physical Journal B - Condensed Matter and Complex Systems 36, 335 (2003).

[Pot03b] M. Potthoff. Self-energy-functional approach to systems of correlated electrons. The European Physical Journal B - Condensed Matter and Complex Systems 32, 429 (2003).

[Pot05] M. Potthoff. Dynamical Variational Principles for Strongly Correlated Electron Systems. In B. Kramer, editor, Advances in Solid State Physics, volume 45 of Advances in Solid State Physics, pages 135-147. Springer Berlin Heidelberg (2005).

[Pot06a] M. Potthoff. Non-perturbative construction of the Luttinger-Ward functional. Cond. Mat. Phys. 9(3), 557 (2006).

[Pot06b] M. Potthoff. Systematics of approximations constructed from dynamical variational principles. AIP Conference Proceedings 816(1), 41 (2006).

[Pot11] M. Potthoff. Static and dynamic variational principles for strongly correlated electron systems. AIP Conference Proceedings 1419(1), 199 (2011).

[Pot12] M. Potthoff. Self-Energy-Functional Theory. In A. Avella and F. Mancini, editors, Strongly Correlated Systems, volume 171 of Springer Series in Solid-State Sciences, pages 303-339. Springer Berlin Heidelberg (2012).

[Pot14] M. Potthoff. Lecture notes of the Autumn School on Correlated Electrons: DMFT at 25: Infinite Dimensions, chapter VCA and DMFT. Forschungszentrum Juelich (2014).

[Pow94] M. J. D. Powell. A Direct Search Optimization Method That Models the Objective and Constraint Functions by Linear Interpolation, pages 51-67. Springer Netherlands, Dordrecht (1994).

[Poz04] K. Pozgajcic. Quantitative aspects of the dynamical impurity approach. E-print arXiv:cond-mat/0407172 (2004).

[PP07] R. Peters and T. Pruschke. Magnetic phases in the correlated Kondo-lattice model. Phys. Rev. B 76, 245101 (2007).

[RA12] M. Raczkowski and F. F. Assaad. Dimensional-Crossover-Driven Mott Transition in the Frustrated Hubbard Model. Phys. Rev. Lett. 109, 126404 (2012).

[RAP15] M. Raczkowski, F. F. Assaad, and L. Pollet. Spin and charge dynamics of a quasi-one-dimensional antiferromagnetic metal. Phys. Rev. B 91, 045137 (2015).

[RCK99] M. J. Rozenberg, R. Chitra, and G. Kotliar. Finite Temperature Mott Transition in the Hubbard Model in Infinite Dimensions. Phys. Rev. Lett. 83, 3498 (1999). 
[RK54] M. A. Ruderman and C. Kittel. Indirect Exchange Coupling of Nuclear Magnetic Moments by Conduction Electrons. Phys. Rev. 96, 99 (1954).

[RKL08] A. N. Rubtsov, M. I. Katsnelson, and A. I. Lichtenstein. Dual fermion approach to nonlocal correlations in the Hubbard model. Phys. Rev. B 77, 033101 (2008).

[RKLG09] A. N. Rubtsov, M. I. Katsnelson, A. I. Lichtenstein, and A. Georges. Dual fermion approach to the two-dimensional Hubbard model: Antiferromagnetic fluctuations and Fermi arcs. Phys. Rev. B 79, 045133 (2009).

[RLRT15] S. Rachel, M. Laubach, J. Reuther, and R. Thomale. Quantum Paramagnet in a $\pi$ Flux Triangular Lattice Hubbard Model. Phys. Rev. Lett. 114, 167201 (2015).

[RSP11] P. Ribeiro, P. D. Sacramento, and K. Penc. Finite-energy spectral function of an anisotropic two-dimensional system of coupled Hubbard chains. Phys. Rev. B 84, 045112 (2011).

[Ruh00] A. Ruhe. Templates for the solution of algebraic eigenvalue problems: $A$ practical guide, chapter 4.1. SIAM (2000).

[Rus63] G. S. Rushbrooke. On the Thermodynamics of the Critical Region for the Ising Problem. The Journal of Chemical Physics 39(3), 842 (1963).

[Saa03] Y. Saad. Iterative methods for sparse linear systems, chapter 6.6. SIAM, 2nd edition (2003).

$\left[\mathrm{SAB}^{+} 79\right]$ F. Steglich, J. Aarts, C. D. Bredl, W. Lieke, D. Meschede, W. Franz, and H. Schäfer. Superconductivity in the Presence of Strong Pauli Paramagnetism: $\mathrm{CeCu}_{2} \mathrm{Si}_{2}$. Phys. Rev. Lett. 43, 1892 (1979).

$\left[\mathrm{SAF}^{+} 11\right]$ O. Stockert, J. Arndt, E. Faulhaber, C. Geibel, H. S. Jeevan, S. Kirchner, M. Loewenhaupt, K. Schmalzl, W. Schmidt, Q. Si, and F. Steglich. Magnetically driven superconductivity in $\mathrm{Ce}_{\mathrm{Cu}} \mathrm{Si}_{2}$. Nat Phys 7(2), 119 (2011).

$\left[\mathrm{SAM}^{+} 01\right]$ N. K. Sato, N. Aso, K. Miyake, R. Shiina, P. Thalmeier, G. Varelogiannis, C. Geibel, F. Steglich, P. Fulde, and T. Komatsubara. Strong coupling between local moments and superconducting 'heavy' electrons in $\mathrm{UPd}_{2} \mathrm{Al}_{3}$. Nature 410(6826), 340 (2001).

[SC90] H. Schweitzer and G. Czycholl. The second order U-perturbation approach to the Anderson Lattice model in one, two and three dimensions. Solid State Communications 74(8), 735 (1990).

[SC91] H. Schweitzer and G. Czycholl. Weak-coupling treatment of the Hubbard model in one, two and three dimensions. Zeitschrift für Physik B Condensed Matter 83(1), 93 (1991).

[Sca95] D. J. Scalapino. The case for $d_{x^{2}-y^{2}}$ pairing in the cuprate superconductors. Phys. Rep. 250, 329 (1995).

[Sch05] U. Schollwöck. The density-matrix renormalization group. Rev. Mod. Phys. 77, 259 (2005).

[Sch11] U. Schollwöck. The density-matrix renormalization group in the age of matrix product states. Annals of Physics 326(1), 96 (2011).

[SDB11] V. Somà, T. Duguet, and C. Barbieri. Ab initio self-consistent GorkovGreen's function calculations of semimagic nuclei: Formalism at second order with a two-nucleon interaction. Phys. Rev. C 84, 064317 (2011). 
[Sén08a] D. Sénéchal. An Introduction in quantum cluster methods. E-print arXiv:0806.2690v2 (2008).

[Sen08b] T. Senthil. Theory of a continuous Mott transition in two dimensions. Phys. Rev. B 78, 045109 (2008).

[Sén12] D. Sénéchal. personal correspondence (2012).

[SFWS84] G. R. Stewart, Z. Fisk, J. O. Willis, and J. L. Smith. Possibility of Coexistence of Bulk Superconductivity and Spin Fluctuations in $\mathrm{UPt}_{3}$. Phys. Rev. Lett. 52, 679 (1984).

[SGR $\left.{ }^{+} 15\right]$ T. Schäfer, F. Geles, D. Rost, G. Rohringer, E. Arrigoni, K. Held, N. Blümer, M. Aichhorn, and A. Toschi. Fate of the false Mott-Hubbard transition in two dimensions. Phys. Rev. B 91, 125109 (2015).

[SHT12] T. Sato, K. Hattori, and H. Tsunetsugu. Transport criticality at the Mott transition in a triangular-lattice Hubbard model. Phys. Rev. B 86, 235137 (2012).

[SLH86] D. J. Scalapino, E. Loh, and J. E. Hirsch. d-wave pairing near a spindensity-wave instability. Phys. Rev. B 34, 8190 (1986).

[SN02] P. Sinjukow and W. Nolting. Exact mapping of periodic Anderson model to Kondo lattice model. Phys. Rev. B 65, 212303 (2002).

$\left[\mathrm{SOY}^{+} 07\right]$ T. Sasaki, H. Oizumi, N. Yoneyama, N. Kobayashi, and N. Toyota. X-ray Irradiation-Induced Carrier Doping Effects in Organic Dimer-Mott Insulators. Journal of the Physical Society of Japan 76(12), 123701 (2007).

[SPP02] D. Sénéchal, D. Perez, and D. Plouffe. Cluster perturbation theory for Hubbard models. Phys. Rev. B 66, 075129 (2002).

[SPPL00] D. Sénéchal, D. Perez, and M. Pioro-Ladrière. Spectral Weight of the Hubbard Model through Cluster Perturbation Theory. Phys. Rev. Lett. 84, 522 (2000).

[SRIS01] Q. Si, S. Rabello, K. Ingersent, and J. L. Smith. Locally critical quantum phase transitions in strongly correlated metals. Nature 413(6858), 804 (2001).

[SRS83] H. Spille, U. Rauchschwalbe, and F. Steglich. Superconductivity in $\mathrm{CeCu}_{2} \mathrm{Si}_{2}$ : Dependence of Tc on Alloying and Stoichiometry. Helvetica Physica Acta 56(1-3), 165 (1983).

[SS10] Q. Si and F. Steglich. Heavy Fermions and Quantum Phase Transitions. Science 329(5996), 1161 (2010).

[SSB $\left.{ }^{+} 00\right]$ M. Schiller, W. Schmidt, E. Balthes, D. Schweitzer, H.-J. Koo, M. H. Whangbo, I. Heinen, T. Klausa, P. Kircher, and W. Strunz. Investigations of the Fermi surface of a new organic metal: (BEDT-TTF $)_{4}\left[\mathrm{Ni}(\mathrm{dto})_{2}\right]$. EPL (Europhysics Letters) 51(1), 82 (2000).

[SSGW95] Z.-P. Shi, R. R. P. Singh, M. P. Gelfand, and Z. Wang. Phase transitions in the symmetric Kondo-lattice model in two and three dimensions. Phys. Rev. B 51, 15630 (1995).

[SSHT12] G. Sordi, P. Sémon, K. Haule, and A.-M. S. Tremblay. Pseudogap temperature as a Widom line in doped Mott insulators. Scientific Reports 2, 547 (2012).

$\left[\mathrm{SSW}^{+} 13\right]$ F. Steglich, O. Stockert, S. Wirth, C. Geibel, H. Q. Yuan, S. Kirchner, and Q. Si. Routes to heavy-fermion superconductivity. Journal of Physics: 
Conference Series 449(1), 012028 (2013).

[ST12] P. Sémon and A.-M. S. Tremblay. Importance of subleading corrections for the Mott critical point. Phys. Rev. B 85, 201101 (2012).

[Ste84] G. R. Stewart. Heavy-fermion systems. Rev. Mod. Phys. 56, 755 (1984).

[Ste14] F. Steglich. Heavy fermions: superconductivity and its relationship to quantum criticality. Philosophical Magazine 94(28), 3259 (2014).

[SV76] B. C. Sales and R. Viswanathan. Demagnetization due to interconfiguration fluctuations in the $R E-C u_{2} S i_{2}$ compounds. Journal of Low Temperature Physics 23(3), 449 (1976).

[SVS04] T. Senthil, M. Vojta, and S. Sachdev. Weak magnetism and non-Fermi liquids near heavy-fermion critical points. Phys. Rev. B 69, 035111 (2004).

[SW66] J. R. Schrieffer and P. A. Wolff. Relation between the Anderson and Kondo Hamiltonians. Phys. Rev. 149, 491 (1966).

[SW12] E. Stoudenmire and S. R. White. Studying Two-Dimensional Systems with the Density Matrix Renormalization Group. Annual Review of Condensed Matter Physics 3(1), 111 (2012).

[SW16] F. Steglich and S. Wirth. Foundations of heavy-fermion superconductivity: lattice Kondo effect and Mott physics. Reports on Progress in Physics 79(8), 084502 (2016).

[SWGK11] M. Sentef, P. Werner, E. Gull, and A. P. Kampf. Charge and spin criticality for the continuous Mott transition in a two-dimensional organic conductor. Phys. Rev. B 84, 165133 (2011).

[SYS $\left.{ }^{+} 05\right]$ T. Sasaki, N. Yoneyama, A. Suzuki, N. Kobayashi, Y. Ikemoto, and H. Kimura. Real Space Imaging of the Metal-Insulator Phase Separation in the Band Width Controlled Organic Mott System $\kappa$-(BEDT$\mathrm{TTF})_{2} \mathrm{Cu} / \mathrm{N}(\mathrm{CN})_{2} / \mathrm{Br}$. Journal of the Physical Society of Japan 74(8), 2351 (2005).

[TKI $\left.{ }^{+} 98\right]$ H. Tou, Y. Kitaoka, K. Ishida, K. Asayama, N. Kimura, Y. Onuki, E. Yamamoto, Y. Haga, and K. Maezawa. Nonunitary Spin-Triplet Superconductivity in $\mathrm{UPt}_{3}$ : Evidence from ${ }^{195} \mathrm{Pt}$ Knight Shift Study. Phys. Rev. Lett. 80, 3129 (1998).

[TMPH14] A. C. Tiegel, S. R. Manmana, T. Pruschke, and A. Honecker. Matrix product state formulation of frequency-space dynamics at finite temperatures. Phys. Rev. B 90, 060406 (2014).

[TVTD11] H. Terletska, J. Vučičević, D. Tanasković, and V. Dobrosavljević. Quantum Critical Transport near the Mott Transition. Phys. Rev. Lett. 107, 026401 (2011).

[VC04] F. Verstraete and J. I. Cirac. Renormalization algorithms for QuantumMany Body Systems in two and higher dimensions. E-print arXiv:condmat/0407066 (2004).

[Vil70] J. Villain. Self consistency of Landau's model in the transition from piezoto ferroelectricity. Solid State Communications 8(5), 295 (1970).

[VLP67] V. Vaks, A. Larkin, and S. Pikin. Self-consistent field method in the description of phase transitions. Zh. Eksp. Teor. Fiz 51 (1967).

[Voj03] M. Vojta. Quantum phase transitions. Reports on Progress in Physics 66(12), 2069 (2003). 
[VTRD15] J. Vučičević, D. Tanasković, M. J. Rozenberg, and V. Dobrosavljević. BadMetal Behavior Reveals Mott Quantum Criticality in Doped Hubbard Models. Phys. Rev. Lett. 114, 246402 (2015).

[Wei07] P. Weiss. L'hypothèse du champ moléculaire et la propriété ferromagnétique. J. Phys. Theor. Appl. 6(1), 661 (1907).

[Whi92] S. R. White. Density matrix formulation for quantum renormalization groups. Phys. Rev. Lett. 69, 2863 (1992).

[WHR15] M. L. Wall, K. R. A. Hazzard, and A. M. Rey. Quantum Magnetism with Ultracold Molecules, chapter 1, pages 3-37. World Scientific (2015).

[Wid65] B. Widom. Surface Tension and Molecular Correlations near the Critical Point. The Journal of Chemical Physics 43(11), 3892 (1965).

[Wid72] B. Widom. Phase Transitions and Critical Phenomena, volume 2. Academic (1972).

[Wil74] K. G. Wilson. Solution of the Spin-1/2 Kondo Hamiltonian. In B. Lundqvist and S. Lundqvist, editors, Collective properties of physical systems, volume 24 of Nobel Symposium, pages 68-75. Nobel Foundation, Academic Press (1974).

[Wil75] K. G. Wilson. The renormalization group: Critical phenomena and the Kondo problem. Rev. Mod. Phys. 47, 773 (1975).

[WLL94] Z. Wang, X.-P. Li, and D.-H. Lee. Kondo insulator to antiferromagnet transition in heavy-fermion compounds. Physica B: Condensed Matter 199200(0), 463 (1994).

[WO07] H. Watanabe and M. Ogata. Fermi-Surface Reconstruction without Breakdown of Kondo Screening at the Quantum Critical Point. Phys. Rev. Lett. 99, 136401 (2007).

[WYTI06] T. Watanabe, H. Yokoyama, Y. Tanaka, and J.-i. Inoue. Superconductivity and a Mott Transition in a Hubbard Model on an Anisotropic Triangular Lattice. Journal of the Physical Society of Japan 75(7), 074707 (2006).

[Xav03] J. C. Xavier. Spin and charge gaps of the half-filled N-leg Kondo ladders. Phys. Rev. B 68, 134422 (2003).

[XD08] J. C. Xavier and E. Dagotto. Robust d-Wave Pairing Correlations in the Heisenberg Kondo Lattice Model. Phys. Rev. Lett. 100, 146403 (2008).

[YGD $\left.{ }^{+} 03\right]$ H. Q. Yuan, F. M. Grosche, M. Deppe, C. Geibel, G. Sparn, and F. Steglich. Observation of Two Distinct Superconducting Phases in $\mathrm{CeCu}_{2} \mathrm{Si}_{2}$. Science 302(5653), 2104 (2003).

[YGML14] L. Yu, Z. Guang-Ming, and Y. Lu. Pairing Symmetry of Heavy Fermion Superconductivity in the Two-Dimensional Kondo-Heisenberg Lattice Model. Chinese Physics Letters 31(8), 087102 (2014).

[YHW11] S. Yan, D. A. Huse, and S. R. White. Spin-Liquid Ground State of the $S=$ 1/2 Kagome Heisenberg Antiferromagnet. Science 332(6034), 1173 (2011).

[YKK09] T. Yoshioka, A. Koga, and N. Kawakami. Quantum Phase Transitions in the Hubbard Model on a Triangular Lattice. Phys. Rev. Lett. 103, 036401 (2009).

[YNK $\left.{ }^{+} 09\right]$ M. Yamashita, N. Nakata, Y. Kasahara, T. Sasaki, N. Yoneyama, N. Kobayashi, S. Fujimoto, T. Shibauchi, and Y. Matsuda. Thermaltransport measurements in a quantum spin-liquid state of the frustrated 
triangular magnet $\kappa-(B E D T-T T F)_{2} C u_{2}(C N)_{3}$. Nat Phys 5(1), 44 (2009).

[YNO $\left.{ }^{+} 08\right]$ S. Yamashita, Y. Nakazawa, M. Oguni, Y. Oshima, H. Nojiri, Y. Shimizu, K. Miyagawa, and K. Kanoda. Thermodynamic properties of a spin-1/2 spin-liquid state in a $\kappa$-type organic salt. Nat Phys 4(6), 459 (2008).

[YNS $\left.{ }^{+} 10\right]$ M. Yamashita, N. Nakata, Y. Senshu, M. Nagata, H. M. Yamamoto, R. Kato, T. Shibauchi, and Y. Matsuda. Highly Mobile Gapless Excitations in a Two-Dimensional Candidate Quantum Spin Liquid. Science 328(5983), 1246 (2010).

[Yos57] K. Yosida. Magnetic Properties of Cu-Mn Alloys. Phys. Rev. 106, 893 (1957).

[YSEO13] A. Yamada, K. Seki, R. Eder, and Y. Ohta. Magnetic properties and Mott transition in the square-lattice Hubbard model with frustration. Phys. Rev. B 88, 075114 (2013).

[ZBG12] M. Zacharias, L. Bartosch, and M. Garst. Mott Metal-Insulator Transition on Compressible Lattices. Phys. Rev. Lett. 109, 176401 (2012).

[ZEAH02] M. G. Zacher, R. Eder, E. Arrigoni, and W. Hanke. Evolution of the stripe phase as a function of doping from a theoretical analysis of angle-resolved photoemission data. Phys. Rev. B 65, 045109 (2002).

[ZRG15] M. Zacharias, A. Rosch, and M. Garst. Critical elasticity at zero and finite temperature. The European Physical Journal Special Topics 224(6), 1021 (2015).

[ZY00] G.-M. Zhang and L. Yu. Kondo singlet state coexisting with antiferromagnetic long-range order: A possible ground state for Kondo insulators. Phys. Rev. B 62, 76 (2000). 